\title{
Picard Lattices of Families of K3 Surfaces
}

\author{
by \\ sarah-marie belcastro
}

A dissertation submitted in partial fulfillment of the requirements for the degree of

Doctor of Philosophy

(Mathematics)

in The University of Michigan

1997

Doctoral Committee:

Professor Igor Dolgachev, Chair

Professor Robert Keener

Visiting Professor Jong Hae Keum

Professor Philip Hanlon

Professor G. Peter Scott 

This dissertation is dedicated to my mathematical mentors, in chronological order:

\author{
Dr. Frank P. Belcastro \\ Mrs. Jan Quere \\ Dr. Curtis Greene \\ Dr. Igor Dolgachev
}

and also to my mathematical guardian angel, Dr. William Cherry. 


\section{ACKNOWLEDGEMENTS}

I am first very grateful to my advisor, Prof. Igor Dolgachev, for giving me huge amounts of help, with the mathematics for this dissertation, with techniques for learning math quickly, and in the job application process. I thank also the veritable army of helpful postdocs who answered my many detailed (and often elementary) questions, namely Dr. William Cherry, Dr. Caryn Werner, and Dr. Everett Howe. I am grateful to Dr. Jong Hae Keum for being the second reader of this document (which is more work than anyone should have to do), and to Profs. Rick Miranda and David Cox for helping me via e-mail. Thanks go also to Pat Shure for giving me the teaching assignment I requested every single time I taught.

I extend gratitude and cat whiskers to Sean Kinlin for supporting me emotionally, making sure I ate correctly, and for caring for Arzachel when I couldn't.

Then, there are those great friends and peers without whose presence I would not have made it through graduate school...

Kim Lindgren helped me through classes 593, 596, and 597, is a kindred spirit, and kept my morale up through the entirety of our first year.

Mark McKerihan studied with me for the Topology QR and worked with me on 631. Moira McDermott worked on homework for 631 and 632 with me, and was (and is!) a role model as the source of all order.

Cathy Kriloff worked with me on courses 614 and 711.

Navah Langmeyer shared my office and left me detailed notes on all the paperwork and fees one must complete in order to graduate, and for job applications.

Moira, Cathy, and Navah all provided me with tips on the job application process and anecdotal evidence from their experiences.

Carolyn Yackel lived nearby and talked with me about teaching, careers, relationships, grad school, philosophy, and life in general. She also did a lot of laundry.

Carol Fan worked on, stressed about, and generally shared the job application process with me... and laughed with me for the whole six years. 


\section{TABLE OF CONTENTS}

DEDICATION ..................... . . . . . . .

ACKNOWLEDGEMENTS .................. . iii

LIST OF TABLES . . . . . . . . . . . . . . . . . v

LIST OF FIGURES . . . . . . . . . . . . . . . . . . . . . vi

LIST OF APPENDICES . . . . . . . . . . . . . . . . vii

\section{CHAPTER}

I. Introduction and Preliminaries . . . . . . . . . . . . . . . . 1

1.1 General Background . . . . . . . . . . . . . . 1

1.2 History of the Problem . . . . . . . . . . . . . . 5 5

1.3 Defining Mirror Symmetry . . . . . . . . . . . . . . . 9

1.4 Calculating $\rho(S) \ldots \ldots \ldots$. . . . . . . . . . . . . . . . . . . . . . . . . . 11

1.5 Somewhat of an Aside: Lattices . . . . . . . . . . . . . . . . 13

1.6 Computing Desingularization Graphs . . . . . . . . . . . . . 19

1.7 Forming Elliptic Fibrations . . . . . . . . . . . . . . . . . 24

1.8 Computing $\operatorname{Pic}(S)$ : Specific Techniques (with an example for

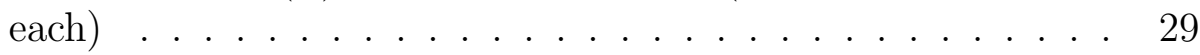

II. Miles Reid's Conjecture . . . . . . . . . . . . . . . . . . . . . . 40

2.1 Statement of the Conjecture . . . . . . . . . . . . . 40

2.2 The $M_{\vec{p}, \vec{\imath}, k}$ Lattices . . . . . . . . . . . . . . . . . . . . 41

2.3 Calculation of $\operatorname{Pic}(S)$ for the $95 \mathrm{~K} 3$ Hypersurfaces Using Reid's Conjecture ................... 46

III. The Detailed Calculations . . . . . . . . . . . . . . . . . 50

$3.1 \quad(1,1,1,1) \ldots \ldots \ldots \ldots \ldots \ldots \ldots \ldots$

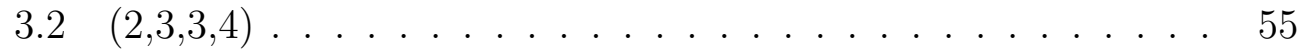

$3.3(1,1,2,2) \ldots \ldots \ldots \ldots \ldots \ldots$ 


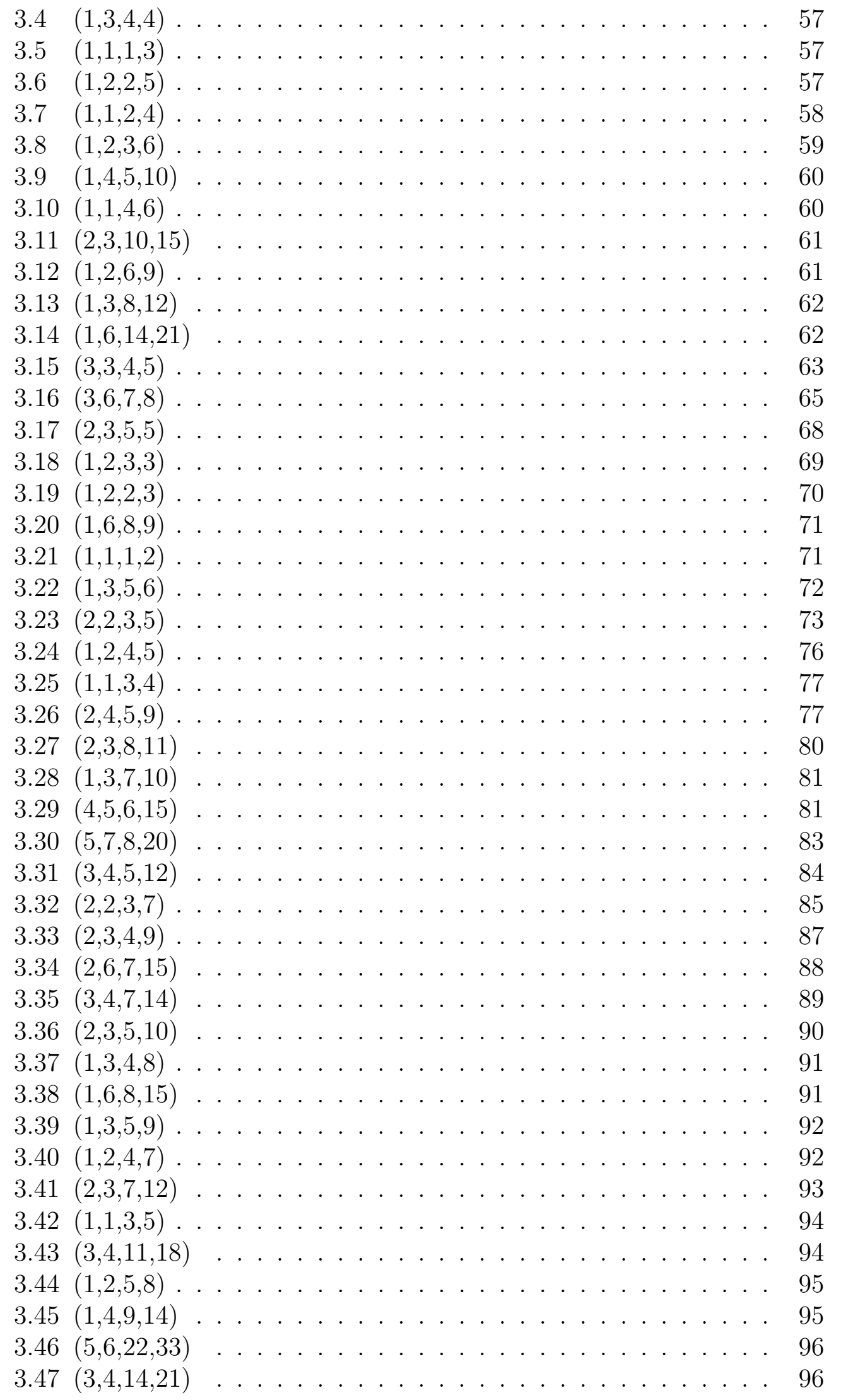




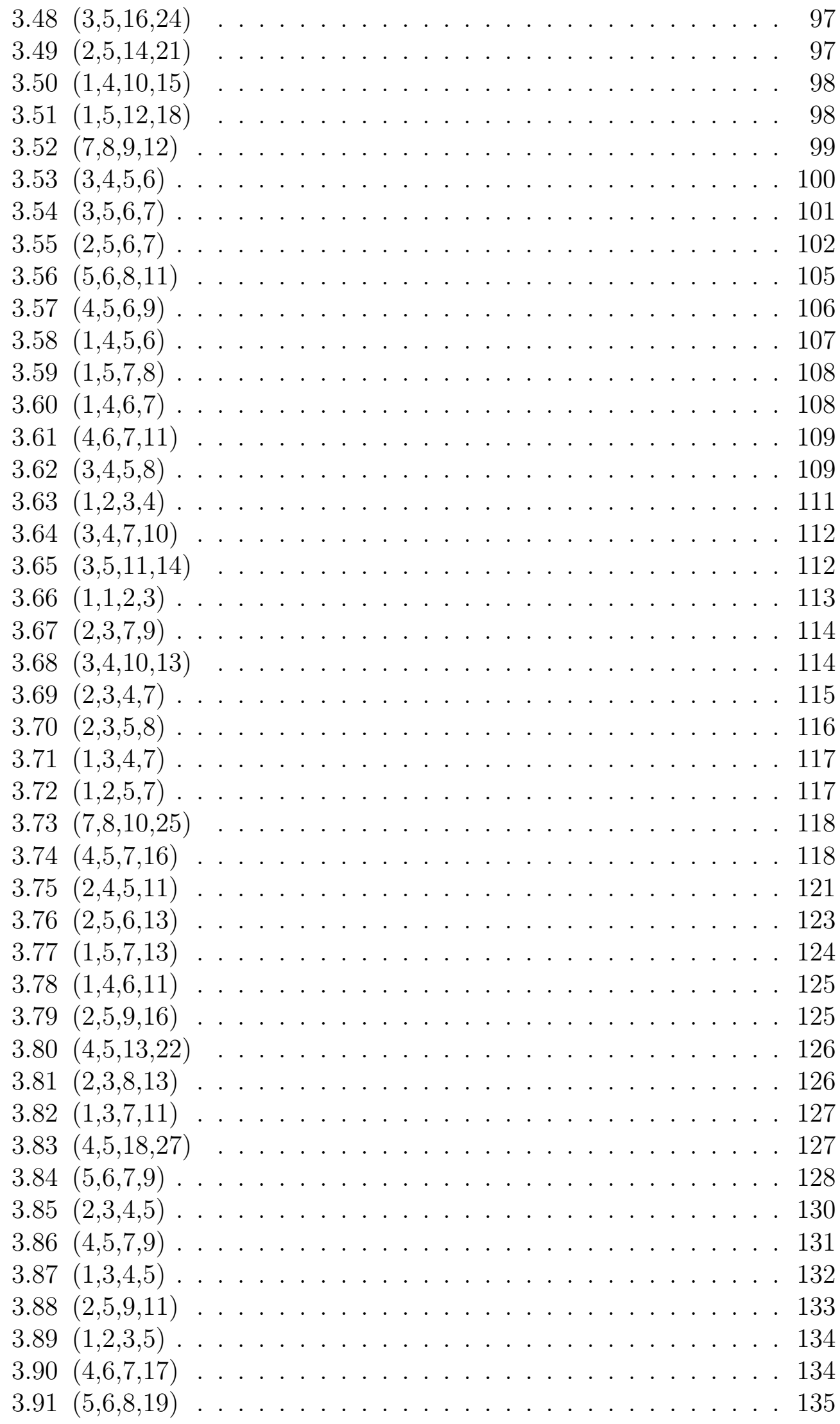


$3.92(3,5,11,19) \ldots \ldots \ldots \ldots$

$3.93(3,4,10,17) \ldots \ldots \ldots \ldots$

$3.94(3,4,5,7) \ldots \ldots \ldots \ldots \ldots \ldots \ldots$

$3.95(2,3,5,7) \ldots \ldots \ldots \ldots \ldots \ldots$

IV. Pic $(S)$ versus $\operatorname{Pic}(\mathbf{J}(S)) \ldots \ldots \ldots \ldots$

4.1 Moduli Spaces of Vector Bundles . . . . . . . . . . . . . . . . 139

4.2 Construction of Vector Bundles . . . . . . . . . . . . . . . 141

4.3 The Correspondence Between $\mathbf{J}(S)$ and Vector Bundles . . 143

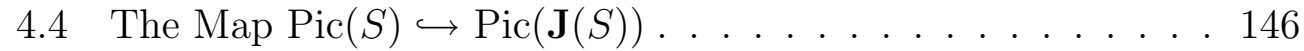

4.5 Further Conjectures ................ . . 148

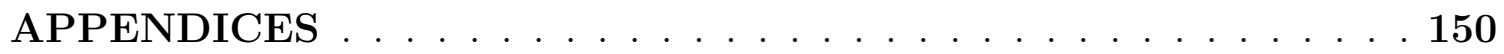

BIBLIOGRAPHY . . . . . . . . . . . . . . . 170 


\section{LIST OF TABLES}

\section{Table}

$1.1 \quad \operatorname{Pic}(S)$ for the 95 Families and Their Mirrors . . . . . . . . . . 6

1.2 Kodaira's Classification of Fibres . . . . . . . . . . . . . 14

1.3 The Dynkin Diagrams . . . . . . . . . . . . . . 15

$1.4 G_{L}$ for each type of Elliptic Fibre . . . . . . . . . . . . 16

$1.5 \quad$ Calculation of $H^{\perp}$ for Number $26 \ldots \ldots \ldots$

1.6 Values of the form $q_{M}$ corresponding to $H$ for Number $26 \ldots$. . . 35

$2.1 \quad$ Forms of the $95 M_{\vec{p}, \vec{\imath}, k} \ldots \ldots \ldots$. . . . . . . . . . 47

3.1 Results for the 95 Families and Their Mirrors . . . . . . . . . . . 50

A.1 Forms and Values for $p \neq 2 \ldots \ldots \ldots 151$

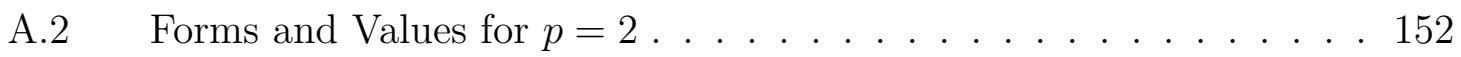

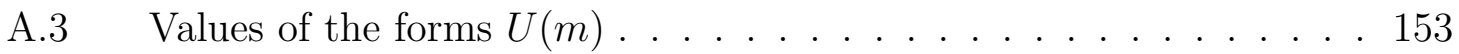

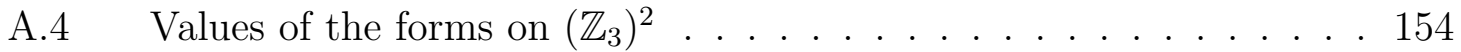

A.5 Values of the forms on $\left(\mathbb{Z}_{3}\right)^{4} \ldots \ldots \ldots . \ldots . \ldots . \ldots 155$ 


\section{LIST OF FIGURES}

\section{Figure}

$1.1 \quad$ A Sample Fibration . . . . . . . . . . . . . . . . . . 3

1.2 Desingularizing a Curve ..................... 4

$1.3 \quad$ Number $26-$ Step $1 \ldots \ldots \ldots . \ldots \ldots$

$1.4 \quad$ Number $26-$ Step $2 \ldots \ldots \ldots$. . . . . . . . . . 26

1.5 Number 26 - Final Step . . . . . . . . . . . . . . . 26

$1.6 \quad$ Number 65 - First Fibration . . . . . . . . . . . . . . . . . . . 29

$1.7 \quad$ Number $52 \ldots \ldots . \ldots \ldots$. . . . . . . . . . . . . 30

1.8 Number 65 - Second Fibration . . . . . . . . . . . . . . . 31

$1.9 \quad$ Number $4 \ldots \ldots \ldots \ldots . \ldots \ldots \ldots \ldots$

$1.10 \quad$ Number $26 \ldots \ldots \ldots \ldots 33$

1.11 Fibration for Number $19 \ldots \ldots \ldots$

2.1 Proof of third relation in Lemma 2.2.1.1 . . . . . . . . . . . . . . 44 


\section{LIST OF APPENDICES}

\section{Appendix}

A. Tables of Forms and Values . . . . . . . . . . . . . . . . . 151

B. Yonemura.ma .................... 156

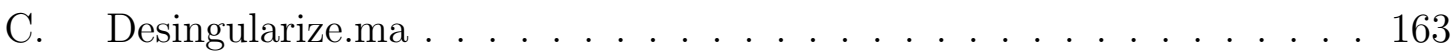

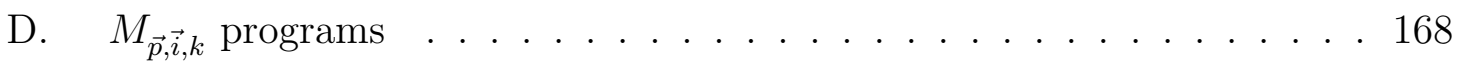




\section{CHAPTER I}

\section{Introduction and Preliminaries}

\subsection{General Background}

\subsubsection{Defining our Terms}

We begin by defining all the terms in the title of the thesis.

1.1.1.1 Definition. A surface is a two-dimensional projective algebraic variety over $\mathbb{C}$.

1.1.1.2 Definition. A hypersurface is a geometric object (variety) described by the zero-set of a single equation in homogenous coordinates, e.g. a two-dimensional variety described by a single equation; an example is $x^{4}+y^{4}+z^{4}+w^{4}=0$.

We form a family of hypersurfaces by varying the coefficients in the equation. For

example, $2 x^{4}+5 y^{4}+3 z^{4}+10 w^{4}=0$ is in the same family as $x^{4}+y^{4}+z^{4}+w^{4}=0$.

1.1.1.3 Definition. The canonical bundle is the top exterior power of the sheaf of holomorphic 1-forms, $=\Lambda^{2} \Omega_{S}$.

1.1.1.4 Definition. A surface is $K 3$ if it is nonsingular, simply-connected and has trivial canonical bundle. 


\subsubsection{The Picard Lattice}

1.1.2.1 Definition. The Picard Group $\operatorname{Pic}(S)$ is the group of isomorphism classes of line bundles on $S$.

For K3 surfaces, this is equivalent to the group of linear equivalence classes of divisors (linear combinations of curves) on the surface. K3 surfaces are all diffeomorphic. Fur-

thermore, for every $\mathrm{K} 3$ surface, $H^{2}(S, \mathbb{Z})$ together with the cup product is naturally a lattice, i.e. a nondegenerate symmetric bilinear form, isomorphic to $\left(E_{8}\right)^{2} \perp(U)^{3}$. One way to distinguish $\mathrm{K} 3$ surfaces is to examine sublattices of $H^{2}(S, \mathbb{Z})$. In our case, $\operatorname{Pic}(S)$ injects into $H^{2}(S, \mathbb{Z})$ and its image is $\operatorname{Pic}(S)=H^{2}(X, \mathbb{Z}) \cap H^{1,1}(X, \mathbb{C})$ (see sections 1.3.2,1.3.3). We determine the Picard Lattice by restricting the bilinear form on $H^{2}(S, \mathbb{Z})$ to $\operatorname{Pic}(S)$. So computing $\operatorname{Pic}(S)$ is essentially computing which 2-cycles are represented by algebraic curves.

In general, it is a difficult and interesting problem to compute $\operatorname{Pic}(S)$ for a random surface.

\subsubsection{Fibrations}

It happens that most of the surfaces we will examine in this thesis have elliptic fibrations.

1.1.3.1 Definition. An elliptic fibration is a map $\pi: S \rightarrow B$ from our surface $S$ to some base curve $B$, such that the general fibre $\pi^{-1}(b)$ is an elliptic curve.

Here is an example, to give the reader a visual image of $\operatorname{Pic}(S)$. We often refer to irreducible fibres and components of reducible fibre as 'vertical' curves.

1.1.3.2 Definition. A section is a divisor which intersects each general fibre at one point. A multisection, or $n$-section, intersects each general fibre at $n$ points. 


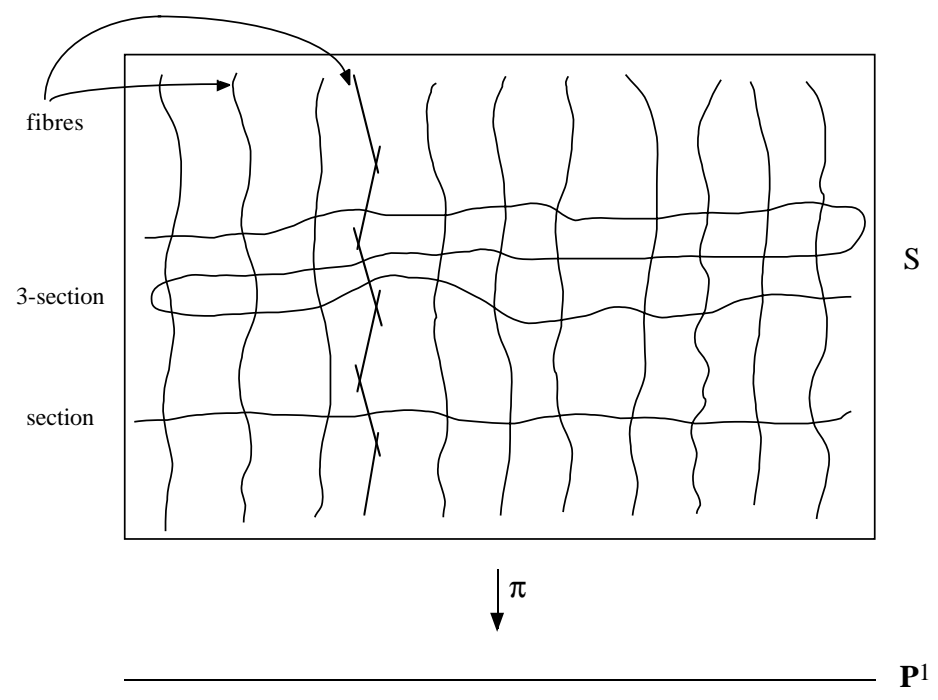

Figure 1.1: A Sample Fibration

Sections and multisections are referred to as 'horizontal' curves, and can intersect themselves and each other (contrary to Figure 1.1, which is drawn for maximal clarity). In Figure 1.1, Pic $(S)$ is roughly the group of different kinds of curves on the surface. All the fibres are linearly equivalent; there is one reducible fibre, whose components contribute to $\operatorname{Pic}(S)$; and then there are sections and multisections. For more on fibrations, see section 1.7. While I have just defined $\operatorname{Pic}(S)$ for nonsingular surfaces, the theory extends to surfaces with $A-D-E$ singularities as well.

\subsubsection{Resolving Singularities}

One way that we obtain reducible fibres is from desingularizing a surface. (A surface is nonsingular if it is a manifold.) The intuitive way to desingularize an object, e.g. for a cusp or node, is to embed the object into a larger-dimensional ambient space so one can "untwist" it in some sense. There is a way to formalize this process $[\mathrm{BPV}, \S I .9]$ : If we have a singular projective surface $S$ in $\mathbb{P}^{n}$, then we embed $S$ in $\mathbb{P}^{n} \times \mathbb{P}^{n-1}$. In particular, we examine an open subset $U$ around a singular point $x=\overrightarrow{x_{i}}$, and consequently $U \times \mathbb{P}^{n-1}$. Let us denote the coordinates on 
$\mathbb{P}^{n}$ by $\left(u_{0}, \ldots, u_{n}\right)$ and those on $\mathbb{P}^{n-1}$ by $\left(v_{0}, \ldots, v_{n-1}\right)$. Now consider the subset $\bar{U}$ of $U \times \mathbb{P}^{n-1}$ defined by the set of equations $\left(u_{i}-x_{i}\right) v_{j}-\left(u_{j}-x_{j}\right) v_{i}=0$, for $i, j=0, n-1$. The projection $\pi: \bar{U} \rightarrow U$ is an isomorphism outside of $x$, and $\pi^{-1}(x) \cong \mathbb{P}^{n-1}$. We often refer to this as "blowing up."

Consider for a moment a curve in $\mathbb{P}^{2}$ with a singular point $x$; geometrically, blowing up is attaching a copy of $\mathbb{P}^{1}$ to $x$, where $t \in \mathbb{P}^{1}$ corresponds to the possible tangent directions at $x$. Then the curve intersects $\mathbb{P}^{1}$ once for each tangent vector at $x$ in the singular model of the curve; in other words, we have "untwisted" the curve along $\mathbb{P}^{1}$. This is pictured in Figure 1.2.
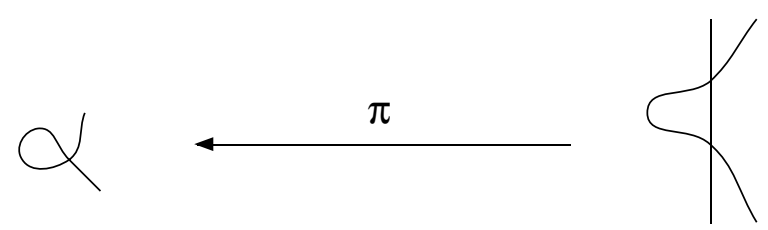

Figure 1.2: Desingularizing a Curve

\subsubsection{Objects of Study}

Here, we examine all families of K3 surfaces which occur as Gorenstein hypersurfaces in weighted projective 4-space. These were classified by Miles Reid; there are 95 of them. This list of surfaces arises in many subfields of algebraic geometry, including singularity theory and the birational geometry of three-folds. The application here will be to mirror symmetry (see section 1.3). For full details on these surfaces, see section 1.6.1.

1.1.5.1 Definition. A weighted projective space is denoted by $\mathbb{P}\left(q_{1}, q_{2}, \ldots, q_{n}\right)$. It is the image of the quotient map

$$
\bar{f}: \mathbb{P}^{3} \rightarrow \mathbb{P}\left(q_{0}, q_{1}, q_{2}, q_{3}\right)=\mathbb{P}^{3} /\left(\mu_{q_{0}} \times \mu_{q_{1}} \times \mu_{q_{2}} \times \mu_{q_{3}}\right) .
$$


If the coordinates of $\mathbb{P}^{3}$ are $x_{0}, x_{1}, x_{2}, x_{3}$, then $\bar{f}$ sends $x_{j} \mapsto \bar{x}_{j}=x_{j}^{q_{j}}$.

So, associated to each of the 95 families of hypersurfaces is a weight-vector $\mathbf{q}=$ $\left(q_{1}, q_{2}, q_{3}, q_{4}\right)$ which describes the weighted projective space $\mathbb{P}\left(q_{1}, q_{2}, q_{3}, q_{4}\right)$ in which it lies. We restrict our attention to a hyperplane of the weighted projective space, i.e. a 3-fold, so our hypersurface is of a three-fold, which is a surface.

\subsection{History of the Problem}

In 1974, V.I. Arnold listed 14 surface singularities (called "exceptional unimodal critical points"). These are of the form $f\left(x_{1}, x_{2}, x_{3}\right)=0$ and can be projectivized and smoothed by changing them to $f\left(x_{1}, x_{2}, x_{3}\right)+x_{0}^{d}=0$; they can then be studied as surfaces. A. Gabrielov calculated the homology of the Milnor Lattice of vanishing cycles for each of these surface singularities. Concurrently, I. Dolgachev was calculating the resolution of singularities for each one. The results of each calculation could be expressed as triples of integers associated to the surface. Interestingly, when Dolgachev's and Gabrielov's data were compared, it was found that the surfaces paired $\left(S, S^{\prime}\right)$ in such a way that the Dolgachev numbers for $S$ were the same as the Gabrielov numbers for $S^{\prime}$ (and, of course, vice-versa). Arnold termed this property a "strange duality." Pinkham, Dolgachev, and Nikulin explained this "strange duality" in the late 1970s, using the theory of K3 surfaces.

These days many mathematicians and physicists discuss "Mirror Symmetry," mainly for Calabi-Yau threefolds. Mirror symmetry pairs two families of manifolds in such a way that several complicated properties are satisfied (see, for example, [Dolg1] for details). Now, Calabi-Yau threefolds are the three-dimensional analogue of K3 surfaces; both have canonical class equivalent to 0 . Naturally it would be interesting to think about what a K3 analogue of the Calabi-Yau threefold mirror symmetry would 
be, so many people investigated this and found that in fact the "strange duality" was such an analogue.

The Problem. A newer question, which arose from studying the K3 mirror symmetry, is as follows: Can one extend this notion of duality to surfaces other than the original 14? In particular, there is Reid's list of 95 families of weighted projective K3-hypersurfaces with Gorenstein singularities, among which the 14 Arnold singularities occur. It would be most interesting to see if all, many, or any of the other surfaces on the list mirror each other. The quick answer to this is that many do, but not all. The results are below, in Table 1.1; details are in Chapter 3. The notation in Table 1.1 is explained in 1.3.3, 1.4, 1.5, 1.6.1, 2.2, and A.0.0.7.

Table 1.1: $\operatorname{Pic}(S)$ for the 95 Families and Their Mirrors

\begin{tabular}{|r|l|l|l|l|l|}
\hline No. & \multicolumn{2}{|c|}{$\operatorname{Rank} \operatorname{Pic}(S)$} & \multicolumn{2}{|c|}{ Mirror Lattice and Family } & Weights \\
\hline 1 & $\rho=1$ & $\langle 4\rangle$ & $\left.\left(E_{8}\right)^{2} \perp-4\right\rangle \perp U$ & 56,73 & $(1,1,1,1)$ \\
2 & $\rho=12$ & $E_{6} \perp D_{4} \perp U(3)$ & $D_{4} \perp A_{2} \perp U(3)$ & not on list & $(2,3,3,4)$ \\
3 & $\rho=4$ & $M_{(1,1,1),(1,1,1), 0}$ & $E_{8} \perp D_{4} \perp A_{2} \perp$ & not on list & $(1,1,2,2)$ \\
& & & $U$ & & \\
4 & $\rho=10$ & $T_{4,4,4}$ & $T_{4,4,4}$ & 4 & $(1,3,4,4)$ \\
5 & $\rho=1$ & $\langle 2\rangle$ & $\left(E_{8}\right)^{2} \perp A_{1} \perp U$ & 52, tetra. & $(1,1,1,3)$ \\
6 & $\rho=6$ & $D_{4} \perp U(2)$ & $D_{8} \perp D_{4} \perp U$ & $26,34,76$ & $(1,2,2,5)$ \\
7 & $\rho=3$ & $M_{(1,1),(1,1), 0}$ & $E_{8} \perp D_{7} \perp U$ & 64 & $(1,1,2,4)$ \\
8 & $\rho=7$ & $M_{(1,1,2,2),(1,1,1,1),-2}$ & $q=w_{3,1}^{1} \perp w_{2,2}^{-1}$ & not on list & $(1,2,3,6)$ \\
9 & $\rho=10$ & $T_{2,5,5}$ & $T_{2,5,5}$ & 9,71 & $(1,4,5,10)$ \\
10 & $\rho=2$ & $U$ & $\left(E_{8}\right)^{2} \perp U$ & $65,46,80$ & $(1,1,4,6)$ \\
11 & $\rho=12$ & $E_{6} \perp D_{4} \perp U$ & $D_{4} \perp A_{2} \perp U$ & 24 & $(2,3,10,15)$ \\
12 & $\rho=6$ & $D_{4} \perp U$ & $E_{8} \perp D_{4} \perp U$ & 27,49 & $(1,2,9,6)$ \\
13 & $\rho=8$ & $E_{6} \perp U$ & $E_{8} \perp A_{2} \perp U$ & 20,59 & $(1,3,8,12)$ \\
14 & $\rho=10$ & $E_{8} \perp U$ & $E_{8} \perp U$ & $14,28,45,51$ & $(1,6,14,21)$ \\
15 & $\rho=14$ & $E_{6} \perp\left(A_{2}\right)^{3} \perp U$ & $\left(A_{2}\right)^{2} \perp U(3)$ & not on list & $(3,3,4,5)$ \\
\hline
\end{tabular}




\begin{tabular}{|c|c|c|c|c|c|}
\hline \multicolumn{6}{|c|}{ continued from previous page } \\
\hline \multirow{2}{*}{$\frac{\text { No. }}{16}$} & \multicolumn{2}{|l|}{ Rank } & \multicolumn{2}{|c|}{ Mirror Lattice and Family } & \multirow{2}{*}{$\frac{\text { Weights }}{(3,6,7,8)}$} \\
\hline & $\rho=16$ & $E_{8} \perp\left(A_{2}\right)^{3} \perp U$ & $A_{2} \perp U(3)$ & not on list & \\
\hline 17 & $\rho=14$ & $T_{2,5,5} \perp A_{4}$ & $A_{4} \perp\left(\begin{array}{cc}2 & 1 \\
1 & -2\end{array}\right)$ & not on list & $(2,3,5,5)$ \\
\hline 18 & $\rho=8$ & $M_{(1,2,2,2),(1,1,1,1),-2}$ & $q=w_{3,2}^{1} \perp w_{3,1}^{1}$ & not on list & $(1,2,3,3)$ \\
\hline 19 & $\rho=7$ & $M_{(1,1,1,1,2),(1,1,1,1,1),-2}$ & $q=v \perp w_{2,3}^{1}$ & not on list & $(1,2,2,3)$ \\
\hline 20 & $\rho=12$ & $E_{8} \perp A_{2} \perp U$ & $E_{6} \perp U$ & 13,72 & $(1,6,8,9)$ \\
\hline 21 & $\rho=2$ & $\left(\begin{array}{cc}2 & 1 \\
1 & -2\end{array}\right)$ & $E_{8} \perp T_{2,5,5}$ & 30,86 & $(1,1,1,2)$ \\
\hline 22 & $\rho=10$ & $E_{6} \perp A_{2} \perp U$ & $E_{6} \perp A_{2} \perp U$ & 22 & $(1,3,5,6)$ \\
\hline 23 & $\rho=11$ & $D_{5} \perp D_{4} \perp U(2)$ & $D_{4} \perp A_{3} \perp U(2)$ & not on list & $(2,2,3,5)$ \\
\hline 24 & $\rho=8$ & $D_{4} \perp A_{2} \perp U$ & $E_{6} \perp D_{4} \perp U$ & 11 & $(1,2,4,5)$ \\
\hline 25 & $\rho=4$ & $A_{2} \perp U$ & $E_{8} \perp E_{6} \perp U$ & $43,48,88$ & $(1,1,3,4)$ \\
\hline 26 & $\rho=14$ & $D_{8} \perp D_{4} \perp U$ & $D_{4} \perp U(2)$ & 6 & $(2,4,5,9)$ \\
\hline 27 & $\rho=14$ & $E_{8} \perp D_{4} \perp U$ & $D_{4} \perp U$ & 12 & $(2,3,8,11)$ \\
\hline 28 & $\rho=10$ & $E_{8} \perp U$ & $E_{8} \perp U$ & $14,28,45,51$ & $(1,3,7,10)$ \\
\hline 29 & $\rho=16$ & $T_{2,5,5} \perp D_{6}$ & $q=w_{5,1}^{-1} \perp\left(w_{2,1}^{-1}\right)^{2}$ & not on list & $(4,5,6,15)$ \\
\hline 30 & $\rho=18$ & $E_{8} \perp T_{2,5,5}$ & $\left(\begin{array}{cc}2 & 1 \\
1 & -2\end{array}\right)$ & 21 & $(5,7,8,20)$ \\
\hline 31 & $\rho=15$ & $E_{6} \perp A_{7} \perp U$ & $q=w_{2,3}^{-1} \perp w_{3,1}^{1}$ & not on list & $(3,4,5,12)$ \\
\hline 32 & $\rho=10$ & $D_{4} \perp D_{4} \perp U(2)$ & $D_{4} \perp D_{4} \perp U(2)$ & 32 & $(2,2,3,7)$ \\
\hline 33 & $\rho=12$ & $\begin{array}{l}M_{(1,1,1,1,2,2,3)} \\
(1,1,1,1,1,1,1),-4\end{array}$ & $\begin{array}{l}q=w_{3,1}^{1} \perp v \perp \\
w_{2,1}^{1} \perp w_{2,1}^{-1}\end{array}$ & not on list & $(2,3,4,9)$ \\
\hline 34 & $\rho=14$ & $D_{8} \perp D_{4} \perp U$ & $D_{4} \perp U(2)$ & 6 & $(2,6,7,15)$ \\
\hline 35 & $\rho=16$ & $E_{8} \perp A_{6} \perp U$ & $M_{(1,2),(1,1), 0}$ & 66 & $(3,4,7,14)$ \\
\hline 36 & $\rho=13$ & $T_{2,5,5} \perp A_{3}$ & $D_{5} \perp\left(\begin{array}{cc}2 & 1 \\
1 & -2\end{array}\right)$ & not on list & $(2,3,5,10)$ \\
\hline 37 & $\rho=9$ & $T_{3,4,4}$ & $T_{2,5,6}$ & 58 & $(1,3,4,8)$ \\
\hline 38 & $\rho=11$ & $E_{8} \perp A_{1} \perp U$ & $E_{7} \perp U$ & 50,82 & $(1,6,8,15)$ \\
\hline 39 & $\rho=9$ & $E_{6} \perp A_{1} \perp U$ & $E_{7} \perp A_{2} \perp U$ & 60 & $(1,3,5,9)$ \\
\hline 40 & $\rho=7$ & $D_{4} \perp A_{1} \perp U$ & $E_{7} \perp D_{4} \perp U$ & 81 & $(1,2,4,7)$ \\
\hline 41 & $\rho=13$ & $E_{6} \perp D_{5} \perp U$ & $A_{3} \perp A_{2} \perp U$ & not on list & $(2,3,7,12)$ \\
\hline 42 & $\rho=3$ & $A_{1} \perp U$ & $E_{8} \perp E_{7} \perp U$ & $68,83,92$ & $(1,1,3,5)$ \\
\hline 43 & $\rho=16$ & $E_{8} \perp E_{6} \perp U$ & $A_{2} \perp U$ & 25 & $(3,4,11,18)$ \\
\hline 44 & $\rho=7$ & $D_{5} \perp U$ & $E_{8} \perp A_{3} \perp U$ & not on list & $(1,2,5,8)$ \\
\hline 45 & $\rho=10$ & $E_{8} \perp U$ & $E_{8} \perp U$ & $14,28,45,51$ & $(1,4,9,14)$ \\
\hline 46 & $\rho=18$ & $E_{8}^{2} \perp U$ & $U$ & 10 & $(5,6,22,33)$ \\
\hline
\end{tabular}




\begin{tabular}{|c|c|c|c|c|c|}
\hline \multicolumn{6}{|c|}{ continued from previous page } \\
\hline \multirow{2}{*}{$\frac{\text { No. }}{47}$} & \multicolumn{2}{|l|}{ Rank } & \multicolumn{2}{|c|}{ Mirror Lattice and Family } & \multirow{2}{*}{$\frac{\text { Weights }}{(3,4,14,21)}$} \\
\hline & $\rho=15$ & $E_{7} \perp E_{6} \perp U$ & $A_{2} \perp A_{1} \perp U$ & not on list & \\
\hline 48 & $\rho=16$ & $E_{8} \perp E_{6} \perp U$ & $A_{2} \perp U$ & 25 & $(3,5,16,24)$ \\
\hline 49 & $\rho=14$ & $E_{8} \perp D_{4} \perp U$ & $D_{4} \perp U$ & 12 & $(2,5,14,21)$ \\
\hline 50 & $\rho=9$ & $E_{7} \perp U$ & $E_{8} \perp A_{1} \perp U$ & 38,77 & $(1,4,10,15)$ \\
\hline 51 & $\rho=10$ & $E_{8} \perp U$ & $E_{8} \perp U$ & $14,28,45,51$ & $(1,5,12,18)$ \\
\hline 52 & $\rho=19$ & $\begin{array}{l}E_{8} \perp D_{9} \perp U \cong \\
\left(E_{8}\right)^{2} \perp\langle-4\rangle \perp U\end{array}$ & $\langle 4\rangle, q=w_{2,2}^{1}$ & 5 & $(7,8,9,12)$ \\
\hline 53 & $\rho=15$ & $\begin{array}{l}M_{(1,2,2,2,3,4)} \\
(1,1,1,1,1,1),-4\end{array}$ & $\begin{array}{l}q=w_{3,2}^{1} \perp w_{3,1}^{1} \perp \\
w_{2,1}^{-1}\end{array}$ & not on list & $(3,4,5,6)$ \\
\hline 54 & $\rho=16$ & $E_{8} \perp\left(A_{2}\right)^{3} \perp U$ & $A_{2} \perp U(3)$ & not on list & $(3,5,6,7)$ \\
\hline 55 & $\rho=15$ & $D_{9} \perp D_{4} \perp U$ & $w_{2,2}^{1} \perp u$ & not on list & $(2,5,6,7)$ \\
\hline 56 & $\rho=19$ & $E_{8}^{2} \perp A_{1} \perp U$ & $\langle 2\rangle, q=w_{2,1}^{1}$ & 1 & $(5,6,8,11)$ \\
\hline 57 & $\rho=17$ & 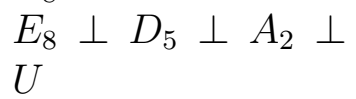 & $w_{2,2}^{5} \perp w_{3,1}^{-1}$ & not on list & $(4,5,6,9)$ \\
\hline 58 & $\rho=11$ & $T_{2,5,6}$ & $T_{3,4,4}$ & 37 & $(1,4,5,6)$ \\
\hline 59 & $\rho=12$ & $E_{8} \perp A_{2} \perp U$ & $E_{6} \perp U$ & 13,72 & $(1,5,7,8)$ \\
\hline 60 & $\rho=11$ & $E_{7} \perp A_{2} \perp U$ & $E_{6} \perp A_{1} \perp U$ & 39 & $(1,4,6,7)$ \\
\hline 61 & $\rho=18$ & $E_{8} \perp D_{8} \perp U$ & $U(2)$ & not on list & $(4,6,7,11)$ \\
\hline 62 & $\rho=16$ & $D_{9} \perp D_{5} \perp U$ & $q=w_{2,2}^{1} \perp w_{2,2}^{5}$ & not on list & $(3,4,5,8)$ \\
\hline 63 & $\rho=8$ & $M_{(1,1,2,3),(1,1,1,1),-2}$ & $T_{2,5,5} \perp\left(A_{1}\right)^{2}$ & not on list & $(1,2,3,4)$ \\
\hline 64 & $\rho=17$ & $E_{8} \perp D_{7} \perp U$ & $M_{(1,1),(1,1), 0}$ & 7 & $(3,4,7,10)$ \\
\hline 65 & $\rho=18$ & $E_{8}^{2} \perp U$ & $U$ & 10 & $(3,5,11,14)$ \\
\hline 66 & $\rho=4$ & $M_{(1,2),(1,1), 0}$ & $E_{8} \perp A_{6} \perp U$ & 35 & $(1,1,2,3)$ \\
\hline 67 & $\rho=14$ & $\begin{array}{l}E_{6}^{2} \perp U \cong E_{8} \perp \\
\left(A_{2}\right)^{2} \perp U\end{array}$ & $\left(A_{2}\right)^{2} \perp U$ & not on list & $(2,3,7,9)$ \\
\hline 68 & $\rho=17$ & $E_{8} \perp E_{7} \perp U$ & $A_{1} \perp U$ & 42 & $(3,4,10,13)$ \\
\hline 69 & $\rho=13$ & $D_{4} \perp A_{7} \perp U$ & $q=w_{2,3}^{-1} \perp v$ & not on list & $(2,3,4,7)$ \\
\hline 70 & $\rho=14$ & $\begin{array}{l}E_{8} \perp A_{2} \perp \\
\left(A_{1}\right)^{2} \perp U\end{array}$ & $q=w_{3,1}^{-1} \perp\left(w_{2,1}^{1}\right)^{2}$ & not on list & $(2,3,5,8)$ \\
\hline 71 & $\rho=10$ & $T_{2,5,5}$ & $T_{2,5,5}$ & 9,71 & $(1,3,4,7)$ \\
\hline 72 & $\rho=8$ & $E_{6} \perp U$ & $E_{8} \perp A_{2} \perp U$ & 20,59 & $(1,2,5,7)$ \\
\hline 73 & $\rho=19$ & $E_{8}^{2} \perp A_{1} \perp U$ & $\langle 2\rangle, q=w_{2,1}^{1}$ & 1 & $(7,8,10,25)$ \\
\hline 74 & $\rho=17$ & $M_{(3,3,4,6),(1,1,1,3),-4}$ & $q=w_{2,3}^{-5}$ & not on list & $(4,5,7,16)$ \\
\hline 75 & $\rho=13$ & $E_{7} \perp\left(A_{1}\right)^{4} \perp U$ & $\left(A_{1}\right)^{5} \perp U$ & not on list & $(2,4,5,11)$ \\
\hline 76 & $\rho=14$ & $D_{8} \perp D_{4} \perp U$ & $D_{4} \perp U(2)$ & 6 & $(2,5,6,13)$ \\
\hline 77 & $\rho=11$ & $E_{8} \perp A_{1} \perp U$ & $E_{7} \perp U$ & 50,82 & $(1,5,7,13)$ \\
\hline
\end{tabular}




\begin{tabular}{|c|c|c|c|c|c|}
\hline \multicolumn{6}{|c|}{ continued from previous page } \\
\hline \multirow{2}{*}{$\frac{\text { No. }}{78}$} & \multicolumn{2}{|l|}{ Rank } & \multicolumn{2}{|c|}{ Mirror Lattice and Family } & \multirow{2}{*}{$\frac{\text { Weights }}{(1,4,6,11)}$} \\
\hline & $\rho=10$ & $E_{7} \perp A_{1} \perp U$ & $E_{7} \perp A_{1} \perp U$ & 78 & \\
\hline 79 & $\rho=15$ & $E_{8} \perp D_{5} \perp U$ & $A_{3} \perp U$ & not on list & $(2,5,9,16)$ \\
\hline 80 & $\rho=18$ & $E_{8}^{2} \perp U$ & $U$ & 10 & $(4,5,13,22)$ \\
\hline 81 & $\rho=13$ & $E_{8} \perp\left(A_{1}\right)^{3} \perp U$ & $D_{4} \perp A_{1} \perp U$ & 40 & $(2,3,8,13)$ \\
\hline 82 & $\rho=9$ & $E_{7} \perp U$ & $E_{8} \perp A_{1} \perp U$ & 38,77 & $(1,3,7,11)$ \\
\hline 83 & $\rho=17$ & $E_{8} \perp E_{7} \perp U$ & $A_{1} \perp U$ & 42 & $(4,5,18,27)$ \\
\hline 84 & $\rho=18$ & $E_{8} \perp A_{8} \perp U$ & $q=w_{3,2}^{-1}$ & not on list & $(5,6,7,9)$ \\
\hline 85 & $\rho=13$ & 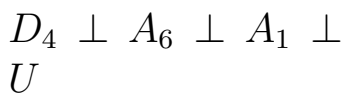 & $D_{4} \perp\langle 14\rangle \perp U$ & not on list & $(2,3,4,5)$ \\
\hline 86 & $\rho=18$ & $E_{8} \perp T_{2,5,5}$ & $\left(\begin{array}{cc}2 & 1 \\
1 & -2\end{array}\right)$ & 21 & $(4,5,7,9)$ \\
\hline 87 & $\rho=10$ & $T_{3,4,5}$ & $T_{3,4,5}$ & 87 & $(1,3,4,5)$ \\
\hline 88 & $\rho=16$ & $E_{8} \perp E_{6} \perp U$ & $A_{2} \perp U$ & not on list & $(2,5,9,11)$ \\
\hline 89 & $\rho=8$ & $M_{(1,2,4),(1,1,2),-2}$ & $A_{10} \perp U$ & not on list & $(1,2,3,5)$ \\
\hline 90 & $\rho=17$ & 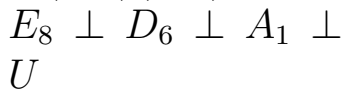 & $A_{1} \perp U(2)$ & not on list & $(4,6,7,17)$ \\
\hline 91 & $\rho=18$ & 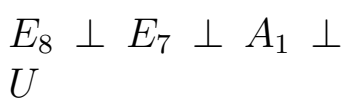 & $q=w_{2,1}^{1} \perp w_{2,1}^{-1}$ & not on list & $(5,6,8,19)$ \\
\hline 92 & $\rho=17$ & $E_{8} \perp E_{7} \perp U$ & $A_{1} \perp U$ & 42 & $(3,5,11,19)$ \\
\hline 93 & $\rho=16$ & $E_{8} \perp D_{6} \perp U$ & $\left(A_{1}\right)^{2} \perp U$ & not on list & $(3,4,10,17)$ \\
\hline 94 & $\rho=16$ & $M_{(2,3,4,6),(1,1,2,2),-4}$ & $q=w_{19,1}^{1}$ & not on list & $(3,4,5,7)$ \\
\hline 95 & $\rho=14$ & $M_{(1,2,4,6),(1,1,2,3),-4}$ & $q=w_{17,1}^{-1}$ & not on list & $(2,3,5,7)$ \\
\hline
\end{tabular}

\subsection{Defining Mirror Symmetry}

\subsubsection{Mirror Symmetry for Threefolds}

Before we answer the main question, we need to discuss the definition of mirror families. We will begin by thinking about Calabi-Yau threefolds for a moment. In the original definition of mirror symmetry, one of the properties that determines when threefolds $X$ and $X^{\prime}$ are mirrors is the following relationship between the Hodge numbers:

$$
h^{1,1}(X)=h^{2,1}\left(X^{\prime}\right), h^{1,1}\left(X^{\prime}\right)=h^{2,1}(X)
$$


This property is equivalent to rotating the Hodge Diamond $90^{\circ}$ counterclockwise and getting the same values back; in other words, we are setting the rank of $\operatorname{Pic}(X)$, which is $h^{1,1}$, equal to the dimension of the moduli space (the tangent space to the space of deformations), which is $h^{2,1}$. This is explained fully in [Dolg1].

\subsubsection{Facts About K3 Surfaces}

Let us examine the Hodge Diamond for K3 surfaces. $H^{2}$ has rank 22, and this decomposes as $h^{2,0}=h^{0,2}=1, h^{1,1}=20$. We have that $h^{0,0}=1=h^{2,2}$ and $h^{2,1}=h^{1,2}=h^{0,1}=h^{1,0}=0$ because K3-surfaces are simply connected (and we use Poincaré and Serre duality) [BPV, p.241]. The Hodge Diamond is invariant under rotation $90^{\circ}$ counterclockwise. In the threefold case, it was $h^{1,1}$ that we examined, and $h^{1,1}$ is now at the center of the Diamond. In particular, both $\operatorname{Pic}(S)$ and the tangent space to the space of deformations lie within $H^{1,1}$, which suggests that we will want to look at its structure when defining our analogous mirror symmetry.

$$
H^{2}(S, \mathbb{Z}) \cong\left(E_{8}\right)^{2} \perp(U)^{3}[\mathbf{B P V}, \text { p.241] }
$$

for any K3 surface; we consider the intersection form on $H^{2}$, which gives us the bilinear form on the lattice.

1.3.2.1 Definition. $U$ is the hyperbolic plane, $\left(\begin{array}{cc}0 & 1 \\ 1 & 0\end{array}\right)$, and we can think of this in geometric terms as the intersection matrix of a section with a fibre.

\subsubsection{Definition of Mirror Symmetry for K3 Surfaces}

To define Mirror Symmetry, we will analyze $H^{2}$. $\operatorname{Pic}(S)$, the group of linear equivalence classes of Cartier divisors, injects into $H^{2}(S, \mathbb{Z})$ for $\mathrm{K} 3$ surfaces $[\mathbf{B P V}$, p.241]. The image of $\operatorname{Pic}(S)$ in $H^{2}(S, \mathbb{Z})$ is the algebraic cycles in $H^{2}(S, \mathbb{Z})$; a theorem 
of Lefschetz says that for $\omega \in H^{2,0}, \int_{\gamma} \omega=0$ if and only if $\gamma$ is algebraic. Therefore $\operatorname{Pic}(S)=H_{\mathbb{R}}^{1,1} \cap H^{2}(S, \mathbb{Z})$. Note that $\operatorname{Pic}(S) \hookrightarrow H^{2}(S, \mathbb{Z})$ is a primitive embedding of lattices, because if $n \gamma \in \operatorname{Pic}(S)$ then $0=\int_{n \gamma} \omega=n \int_{\gamma} \omega$ so $\int_{\gamma} \omega=0$ and $\gamma \in \operatorname{Pic}(S)$. Thus, there is no torsion in the image of $\operatorname{Pic}(S)$ in $H^{2}(S, \mathbb{Z})$, so we may consider $\operatorname{Pic}(S)$ as a lattice, and call it the Picard lattice.

1.3.3.1 Definition. Two surfaces form a mirror pair $\left(S, S^{\prime}\right)$ if

$$
\operatorname{Pic}(S)_{H^{2}(S, \mathbb{Z})}^{\perp}=\operatorname{Pic}(\check{S}) \perp U \text { as lattices. }
$$

Just as we call $\operatorname{Pic}(S)$ the Picard Lattice, we refer to $\operatorname{Pic}(\check{S})$ as the mirror lattice.

\subsection{Calculating $\rho(S)$}

In 1979, M. Reid classified and listed all families of weighted projective Gorenstein K3-hypersurfaces, but he never published this list. [Yonemura] lists the weightvectors for the associated weighted projective spaces for each of the 95 families of surfaces. Let $\pi: \tilde{S} \rightarrow S$ be the resolution of singularities. The first thing we need to determine is $\rho(\tilde{S})$, the rank of the Picard lattice. We know that $S$ has a natural desingularization in terms of $A_{p_{j}}$ singularities (see Section 1.6.2).

1.4.0.2 Theorem. $\rho(\tilde{S})=1+\Sigma_{j} p_{j}$, where the $p_{j}$ are the types of the cyclic singularities of $S$.

Proof of 1.4.0.2. We combine the two lemmas below; the first gives the rank of $\operatorname{Pic}(S)$ before desingularizing, and the second addresses the contribution to $\operatorname{Pic}(\tilde{S})$ by desingularizing. 
1.4.0.3 Lemma. Let $S$ be a generic surface in one of the 95 families. Then $\rho(S)=1$.

Proof of 1.4.0.3. The degree of the generic surface is the weight of a variable times its degree in the equation. In our case, the degree is $s=q_{0}+q_{1}+q_{2}+q_{3}$ (see Section 1.6.1). In $[\mathbf{C o x}]$, the author examines the cup product $c: H^{1}\left(S, T_{S}\right)_{0} \times H^{2,0}(S) \rightarrow$ $H^{1,1}(S)$ where $H^{1}\left(S, T_{S}\right)_{0}$ represents the variations in $S$ from varying the coefficients in its equation. He concludes that if this map is surjective, then for generic $S, r k(\operatorname{Pic}(S))=1$. We will now show that this map is surjective when the degree equals $s$. Denote by $R=\bigoplus R^{s}$ the graded Jacobian ring $\mathbb{C}\left[x_{0}, x_{1}, x_{2}, x_{3}\right] / \overline{\partial f}$. Then, $H^{2,0}(S)=R^{0}, H^{1,1}(S)=R^{s}$ by $\left[\right.$ Dolg3, 4.3.3]; $H^{1}\left(S, T_{S}\right)_{0}=H^{1}\left(S, \Omega_{S}^{1}\right)_{0}=\left(H^{1,1}\right)_{0}=$ the primitive part of $H^{1,1}$, which is all of $H^{1,1}$ on a surface and so $H^{1}\left(S, T_{S}\right)=R^{s}$. Because $c$ is just the multiplication in $R$, it is an isomorphism because $R^{0}$ is generated by 1 . Thus $c$ is surjective.

1.4.0.4 Lemma. The contribution to $\operatorname{Pic}(\tilde{S})$ from desingularizing $S$ is $\Sigma_{j} p_{j}$ where the $p_{j}$ are the types of the $A_{p_{j}}$ singularities of $S$.

Proof of 1.4.0.4. Yonemura calculates (and we verify by computer (see section 1.6)) a minimal desingularization of each surface. Each singularity is of type $A_{p_{j}}$ (see section 1.6.1), and so its minimal desingularization produces $p_{j}+1(-2)$-curves $[\mathbf{B P V}$, $\S$ V.7]. It is clear that these are independent by examining their intersections with each other. We know that before desingularizing, $\operatorname{Pic}(S) \cong \mathbb{Z}$; when we desingularize, we have, up to finite index, the orthogonal decomposition $\operatorname{Pic}(\tilde{S})=\Sigma_{j} A_{p_{j}} \oplus \pi^{*} \operatorname{Pic}(S)$. Henceforth we will abuse notation by referring to the rank of the Picard Lattice of the nonsingular model of $S$ as $\rho(S)$. 
1.4.0.5 Remark. It is interesting to note that there are not many results on the Picard number of a family of toric hypersurfaces. If there were results for more general toric hypersurfaces, one could apply most of the techniques listed later to a wider class of objects.

\subsection{Somewhat of an Aside: Lattices}

This section summarizes general background on lattices. Further information on specific types of lattices may be found in Chapter 2 and Appendix 1.

1.5.0.6 Definition. We define a lattice as a pair $(L, b)$ where $L$ is a finite-rank free $\mathbb{Z}$-module and $b$ is a $\mathbb{Z}$-valued nondegenerate symmetric bilinear form.

We will only consider even lattices, those where $b(l, l)$ is even for all $l$. We also denote $b(l, l)$ by $\langle l, l\rangle$.

1 5.0.7 Definition. The discriminant of a lattice is the determinant of the matrix of the associated bilinear form.

\subsubsection{The Kodaira Classification of Fibres}

Later we will be searching for elliptic fibrations of our K3 surfaces. It would be useful to know what possibilities there are for elliptic fibres; all of these correspond to lattices. Good references for proof of this classification are [BPV] and [Miranda]. In Table 1.2 we have the different types of fibres; we list the name, description, and a graph where applicable.

On the graphs, each vertex represents a curve and each edge represents an intersection between two curves. A label on a vertex corresponds to the multiplicity of that curve in the fibre. Where there is no label, the curve has multiplicity one. The 


\begin{tabular}{|c|c|c|}
\hline Type & Description & Diagram \\
\hline$I_{0}$ & smooth elliptic curve & \\
\hline$I_{1}$ & nodal rational curve & \\
\hline$I_{n}$ & $\begin{array}{l}n \geq 2 ; n \text { smooth rational curves forming a } \\
\left.\text { cycle (graph } \tilde{A}_{n-1}\right)\end{array}$ & \\
\hline$I_{n}^{\star}$ & $\begin{array}{l}n+5 \text { smooth rational curves forming graph } \\
\tilde{D}_{n+4}(n \geq 0)\end{array}$ & \\
\hline $\begin{array}{l}{ }_{m} I_{n} \\
I I\end{array}$ & $\begin{array}{l}n \geq 0 ; I_{n} \text { with each curve of multiplicity } m \\
\text { cuspidal rational curve }\end{array}$ & \\
\hline$I I^{\star}$ & 9 smooth rational curves forming graph $\tilde{E}_{8}$ & 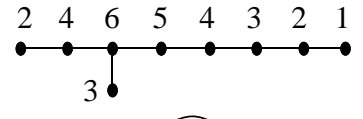 \\
\hline$I I I$ & $\begin{array}{l}\text { two smooth rational curves intersecting once } \\
\text { with multiplicity two }\end{array}$ & \\
\hline$I I I^{\star}$ & 8 smooth rational curves forming graph $\tilde{E}_{7}$ & 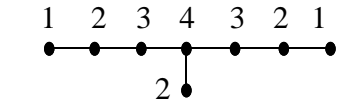 \\
\hline$I V$ & $\begin{array}{l}\text { three smooth rational curves intersecting in } \\
\text { one point }\end{array}$ & \\
\hline$I V^{\star}$ & 7 smooth rational curves forming graph $\tilde{E}_{6}$ & 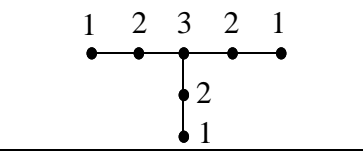 \\
\hline
\end{tabular}

Table 1.2: Kodaira's Classification of Fibres

self-intersection of each curve in a reducible fibre is -2 because they're rational (the irreducible fibres have self-intersection 0). Some of these fibres will never occur on our 95 surfaces; K3 surfaces do not have multiple fibres [BPV], so we will never see an ${ }_{m} I_{n}$.

\subsubsection{Dynkin Diagrams}

The graphs in Table 1.2 are the extended Dynkin diagrams. There are also "plain" Dynkin diagrams, some of which are presented in Table 1.3; a good reference for these is [Humphreys]. The extended Dynkin diagrams are formed from the Dynkin diagrams by adding a curve with multiplicity one. I am sure that by now the 
reader is wondering, "What do these have to do with lattices?" Of course, there is a relation: Form the incidence matrix $A$ for the graph by letting the rows/columns be indexed by the curves (vertices). Entry $a_{i j}=1$ if curves $i$ and $j$ intersect (if there's an edge between vertices $i$ and $j$ ) and $a_{i j}=0$ if curves $i$ and $j$ do not intersect (if there is no edge between vertices $i$ and $j$ ). Set the diagonal entries $a_{i i}=-2$ because that is the self-intersection of each curve. The incidence matrix is symmetric and nondegenerate, and so represents the bilinear form for a lattice associated to each Dynkin Diagram.

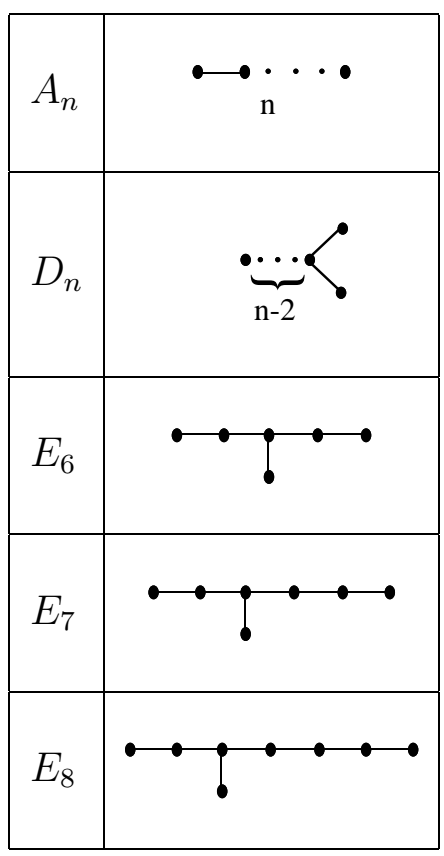

Table 1.3: The Dynkin Diagrams

\subsubsection{Nikulin's results}

Almost all of our lattice techniques and facts are contained in [Nikulin], an awesome (though densely written) paper. Lattices are classified by their discriminant quadratic forms $q_{L}$ (often referred to simply as $q$ ). We define $q$ by $q_{L}(x)=\langle x, x\rangle$ $\bmod 2 \mathbb{Z}$; note that $q$ is the quadratic form associated to the bilinear form $b$ which 
defines the lattice $L$. The discriminant form is defined on the discriminant group $G_{L}=L^{\star} / L$, where $L^{\star}=\operatorname{Hom}(L, \mathbb{Z})$. We may also view this as

$$
L^{\star}=\left\{x \in L \otimes_{\mathbb{Z}} \mathbb{Q} \mid\langle x, l\rangle \in \mathbb{Z} \text { for all } l \in L\right\} .
$$

We list $G_{L}$ for the applicable types of fibres in Table 1.4.

\begin{tabular}{|l|l|c|}
\hline$L$ & Type & $G_{L}$ \\
\hline & $I_{0}$ & $\{0\}$ \\
\hline$A_{n}$ & $I_{n+1}, I I I, I V$ & $\mathbb{Z}_{n}$ \\
\hline$D_{n}$ & $I_{n-4}^{\star}$ & $\begin{array}{c}n \text { even, } \mathbb{Z}_{2} \oplus \mathbb{Z}_{2} \\
n \text { odd, } \mathbb{Z}_{4}\end{array}$ \\
\hline$E_{6}$ & $I V^{\star}$ & $\mathbb{Z}_{3}$ \\
\hline$E_{7}$ & $I I I^{\star}$ & $\mathbb{Z}_{2}$ \\
\hline$E_{8}$ & $I I^{\star}$ & $\{0\}$ \\
\hline
\end{tabular}

Table 1.4: $G_{L}$ for each type of Elliptic Fibre

\section{Classification of the Forms}

We will define three classes of forms on $G_{L}: w_{p, k}^{\epsilon}, u_{k}, v_{k}$. An excellent explanation of these forms can be found in [Brieskorn].

\subsubsection{Definition. We define the quadratic form $w_{p, k}^{\epsilon}$ on $\mathbb{Z}_{p^{k}}$.}

Case 1: For $p \neq 2$, it is the form with generator value $q(1)=a p^{-k}(\bmod 2 \mathbb{Z})$, where $a$ is determined by the quadratic residues of $p$. A quadratic residue is denoted $\left(\frac{a}{p}\right)$ and this notation is called the Jacobi-Legendre symbol. It is defined as follows: is there a solution to the equation $x^{2}=a$ where $x$ is (modulo $\mathrm{p}$ )? If so, we set the symbol equal to 1 , and if not, to -1 . Thus we only allow $\epsilon \in\{ \pm 1\}$. For $\epsilon=1$ we choose $a$ to be the smallest positive even number with a quadratic residue; for $\epsilon=-1$ we choose $a$ to be the smallest positive even number without a quadratic residue. 
Case 2: For $p=2$, there are more possibilities. For $k=1, \epsilon \in\{ \pm 1\}, w_{2,1}^{\epsilon}$ is defined as the form with generator value $q(1)=\epsilon / 2$. For $k \geq 1$ and $\epsilon \in\{ \pm 1, \pm 5\}, w_{2, k}^{\epsilon}$ is defined as the form with generator value $q(1)=\epsilon / 2^{k}$.

1.5.3.2 Definition. We define the forms $u_{k}, v_{k}$ on $\mathbb{Z}_{2^{k}} \oplus \mathbb{Z}_{2^{k}}$. We will describe these forms via their matrices, which are

$$
u_{k}=2^{-k}\left(\begin{array}{cc}
0 & 1 \\
1 & 0
\end{array}\right) \quad v_{k}=2^{-k}\left(\begin{array}{cc}
2 & 1 \\
1 & 2
\end{array}\right) .
$$

Examples of each of these types of forms are in Appendix 1.

\section{Notions of Isomorphism}

The following theorem is a combination of [Nikulin, 1.8.1,1.8.2].

\subsubsection{Theorem}

(i) Every nontrivial, nondegenerate irreducible quadratic form on a finite abelian group is isomorphic to one of $u_{k}, v_{k}, w_{p, k}^{\epsilon}$.

(ii) Every nondegenerate quadratic form on a finite abelian group is isomorphic to an orthogonal direct sum of $u_{k}, v_{k}, w_{p, k}^{\epsilon}$.

(iii) This representation of a quadratic form is not unique. The isomorphism relations between the forms are as follows:

$$
\begin{gathered}
p \neq 2, w_{p, k}^{\epsilon} \perp w_{p, k}^{\epsilon} \cong w_{p, k}^{\epsilon^{\prime}} \perp w_{p, k}^{\epsilon^{\prime}} \\
u_{k} \perp u_{k} \cong v_{k} \perp v_{k} \\
w_{2, k}^{\epsilon} \perp w_{2, k}^{\epsilon^{\prime}} \cong w_{2, k}^{5 \epsilon} \perp w_{2, k}^{5 \epsilon^{\prime}} \\
\text { for } \epsilon^{\prime} \equiv \epsilon(\bmod 4), v_{k} \perp w_{2, k}^{-5 \epsilon^{\prime}} \cong w_{2, k}^{\epsilon} \perp w_{2, k}^{\epsilon} \perp w_{2, k}^{\epsilon^{\prime}}
\end{gathered}
$$




$$
\begin{gathered}
\text { for } \epsilon^{\prime} \equiv-\epsilon(\bmod 4), u_{k} \perp w_{2, k}^{-\epsilon^{\prime}} \cong w_{2, k}^{\epsilon} \perp w_{2, k}^{\epsilon} \perp w_{2, k}^{\epsilon^{\prime}} \\
v_{k} \perp w_{2, k+1}^{\epsilon} \cong u_{k} \perp w_{2, k+1}^{5 \epsilon} \\
v_{k+1} \perp w_{2, k}^{\epsilon} \cong u_{k+1} \perp w_{2, k}^{5 \epsilon} \\
w_{2, k}^{\epsilon} \perp w_{2, k+1}^{\epsilon^{\prime}} \cong w_{2, k}^{\epsilon+2 \epsilon^{\prime}} \perp w_{2, k+1}^{5\left(\epsilon^{\prime}-2 \epsilon\right)} \\
w_{2, k}^{\epsilon} \perp w_{2, k+2}^{\epsilon^{\prime}} \cong w_{2, k}^{5 \epsilon} \perp w_{2, k+2}^{5 \epsilon^{\prime}}
\end{gathered}
$$

Of course, there are also isomorphism relations which arise from these relations.

\section{Orthogonality and Mirrors}

There are three contexts in which we use orthogonality.

Context 1. One is when we use the $\perp$ symbol between two lattices, e.g. $L=A_{k} \perp$ $D_{m}$, which indicates that $L$ has an orthogonal decomposition as a block-matrix.

Context 2. We define orthogonality of an element $a \in G_{L}$ to a subgroup $H \subset G_{L}$ as when $q(a)-q([H]+a)=0$ is satisfied.

Context 3. The final context is when we take the orthogonal complement of a lattice within a unimodular (trivial $q$ ) lattice.

Context 3 is the sense in which we compute mirrors. For a lattice embedded primitively into a unimodular lattice (this is the case for $\operatorname{Pic}(S)$ and $H^{2}(S, \mathbb{Z})$ ), $L_{H^{2}(S, \mathbb{Z})}^{\perp}=M$ if and only if $q_{L}=-q_{M}$ [Nikulin, 1.6.2]. We determine $-q(M)$ by multiplying the value of $q$ on each element of $G_{M}$ by -1 , and then determining what form this set of values corresponds to. In practice, this is simple when using the tables in Appendix 1. 


\section{Existence and Uniqueness}

This leaves us with only a few questions: how can we be sure that a mirror to a given lattice exists? When we have determined a form or a mirror-form, how can we be sure that this is unique? Fortunately, Nikulin also helps us with these questions [Nikulin, 1.10.2, 1.13.3]. Notice that the signature of $H^{2}(S, \mathbb{Z})$ is $(3,19)$; the signature of $\operatorname{Pic}(S)$ will always be $(1, \rho-1)$ by the Hodge Index Theorem, and the signature of the mirror-lattice $\operatorname{Pic}(\check{S})=M$ will be $(1,19-\rho)$ (the remaining $U$ gives us the missing $(1,1))$. We refer to the first coordinate of the signature as $t_{+}$ and the second coordinate as $t_{-}$.

1.5.3.4 Theorem [Nikulin, 1.10.2] If $20-\rho$ is greater than the number of generators of $G_{M}$ and if $\rho-18 \equiv t_{L+}-t_{L-}(\bmod 8)$ for some known-to-exist lattice $L$ with $q_{M}=q_{L}$, then the mirror-lattice exists.

1.5.3.5 Theorem [Nikulin, 1.13.3] If, additionally, $18-\rho$ is greater than the number of generators of $G_{M}$ and if $t_{M+}, t_{M-} \geq 1$, then the mirror-lattice is unique.

If either of these criteria do not hold, it does not mean that the mirror-lattice does not exist or that it is not unique, but that we need different criteria. This will be exhibited in the very few cases in which we need it.

1.5.3.6 Theorem [Nikulin, 1.12.3] If $\rho+2$ is is greater than the number of generators of $G_{M}$, then the mirror-lattice $M$ embeds primitively into $H^{2}(S, \mathbb{Z})$.

\subsection{Computing Desingularization Graphs}

Generally, we use the toric description of each hypersurface to desingularize it, and use this desingularization to find an elliptic fibration. Why does this help in 
computing $\operatorname{Pic}(S)$ ? When the computer desingularizes the hypersurface, it outputs a graph which depicts each component of a resolved singularity and each face as a vertex, and intersections between them as edges. The intersections are determined by the desingularization and the covering relations in the face-lattice. The incidence matrix of the graph is a bilinear form, which is a lattice. We now have a lattice, of the same rank as $\operatorname{Pic}(S)$, generated by curves on the surface, and which is certainly a sublattice of $\operatorname{Pic}(S)$. In this section, I will describe the process by which we obtain the desingularization graph.

\subsubsection{The Toric Description}

The field of toric varieties is a way to use combinatorial language to describe algebraic varieties. Usually a toric variety is described by polyhedral cones or polytopes in $\mathbb{Z}^{n}$, where each lattice point in the object corresponds to a monomial in the coordinate ring of an affine piece (in the gluing sense) of the algebraic variety. Excellent references for basic information on toric varieties are [Fulton] and [Danilov].

\section{Weighted Projective Space}

We denote weighted projective space as $\mathbb{P}\left(q_{1}, q_{2}, \ldots, q_{n}\right)$. A weighted projective space is different from the projective space $\mathbb{P}^{n-1}$ only in that the coordinates are assigned weights, i.e. the variable $x_{i}$ has weight $q_{i}$. So, associated to each of the 95 families of hypersurfaces is a weight-vector $\mathbf{q}=\left(q_{1}, q_{2}, q_{3}, q_{4}\right)$ which describes the weighted projective space $\mathbb{P}\left(q_{1}, q_{2}, q_{3}, q_{4}\right)$ in which it lies. We denote $\sum q_{i}$ by $s$.

Just as we view $\mathbb{P}^{2}$ as a toric variety defined by the fan with edges

$$
\{(1,0),(0,1),(-1,-1)\}
$$


and $\mathbb{P}^{n-1}$ as a toric variety defined by the fan with edges

$$
\{(1, \ldots, 0), \ldots,(0, \ldots, 1),(-1,-1, \ldots,-1)\}
$$

we can consider the weighted projective space

$\mathbb{P}\left(q_{1}, q_{2}, \ldots, q_{n}\right)$ as a toric variety defined by the fan with edges

$$
\left\{\left(q_{n}, \ldots, 0\right), \ldots,\left(0, \ldots, q_{n}\right),\left(-q_{1}, \ldots,-q_{n-1}\right)\right\}
$$

We also can view $\mathbb{P}^{n-1}$ as a toric variety defined by the polytope

$$
\operatorname{conv}\{(1, \ldots, 0), \ldots,(0, \ldots, 1)\}
$$

similarly, we can view $\mathbb{P}\left(q_{1}, q_{2}, \ldots, q_{n}\right)$ as a toric variety defined by the polytope

$$
\operatorname{conv}\left\{\left(\Pi q_{i} / q_{1}, \ldots, 0\right), \ldots,\left(0, \ldots, \Pi q_{i} / q_{n}\right)\right\}
$$

For details of these constructions, see [Fulton, pp. 25-27,35] and [Dolg3, 1.2.5].

\section{Our Objects}

All of the 95 hypersurfaces are Gorenstein, i.e. they have the property that $\operatorname{deg}(S)=s$; this comes from the adjunction formula in weighted projective space (see $\left[\right.$ Dolg3]). If we have a polytope $\operatorname{conv}\left\{\overrightarrow{p_{1}}, \ldots, \overrightarrow{p_{n}}\right\}$, defined by its extremal points $\overrightarrow{p_{1}}, \ldots, \overrightarrow{p_{n}}$, then the hypersurface associated to this polytope has equation $\sum_{i} \overrightarrow{x_{i}} \overrightarrow{p_{i}}$. Thus, as we are looking for hypersurfaces of degree $s$, we want our monomials to be of degree $s$, or equivalently, we want our lattice points to lie in $\left\{\vec{x} \in \mathbb{R}^{4} \mid \sum q_{i} x_{i}=s\right\}$. Therefore, we consider the following object:

$$
\operatorname{conv}\left\{\left(s / q_{1}, \ldots, 0\right), \ldots,\left(0, \ldots, s / q_{n}\right)\right\}
$$

is a rational polytope, which is possibly integral (but generally is not). We wish to take the convex hull of all integral points of this rational polytope in order to view the associated hypersurface as a toric variety. 
First, however, notice that the condition $\sum q_{i} x_{i}=s$ means that the rational polytope lies in a hyperplane of $\mathbb{R}^{4}$. Using this condition, we may consider this object as lying in $\mathbb{R}^{3}$. After transforming the rational polytope to $\mathbb{R}^{3}$, keeping the internal lattice fixed, we take the convex hull of all integral points, hereafter referred to as the Newton polytope.

Many of the combinatorial features of the polytope correspond to geometric aspects of the hypersurface. For example, a face of the polytope corresponds to a curve on the surface, and the number of lattice points on the face corresponds to the genus of the corresponding curve. An edge corresponds to an open set in the surface.

\subsubsection{Desingularizing Hypersurfaces}

The desingularization of $S$ is simplified because, as we show in this section, all singularities of $S$ lie on the edges of the polytope.

Case 1 - Vertices. Almost all equations in the family of hypersurfaces are nondegenerate with respect to the Newton Polytope [Khovanskiı̌, § 2]. If one happens to choose a degenerate equation, one can always change the coefficients to obtain a nondegenerate equation. It follows that $S$ intersects each toroidal orbit transversally. This means that $S$ avoids the vertices of the polytope as transversal intersections have lesser dimension than either of the intersecting bodies and vertices have dimension 0 .

Case 2 - Faces. A face is 2-dimensional, so its dual is 1-dimensional and must be a curve. Whether this curve is singular or not is immaterial to whether there are singularities of $S$.

Case 3 - Edges. This leaves only the edges. The dual to the 1-dim edge is $2-\mathbb{R}$ dimensional, i.e. a plane. This plane is spanned by the normal vectors $v_{1}, v_{2}$ to 
the two faces $F_{1}, F_{2}$ which intersect to form the edge.

1.6.2.1 Fact [Fulton, p.47]. On a plane, there are only $A_{n}$-type singularities possible. We can see this because all singularities of the plane occur on the boundary. Examine the lattice in the plane dual to the edge. The vectors $v_{1}, v_{2}$ do not necessarily generate the lattice; we determine $n$ by the number of additional vectors needed to generate the lattice. The multiplicity of an edge singularity is the number of lattice points on the edge, minus one.

This gives us series of curves corresponding to the singularities on the edges. In order to see how these curves intersect with other curves on the surface, we look at faces. Faces correspond to curves (of arithmetic genus equal to the number of interior points), and thus because every edge is the intersection of two faces, every singularity connects 2 curves. In other words, each face corresponds to a curve joining the singularities corresponding to each edge.

\subsubsection{But How Do We Actually Do It?}

It is of course possible to perform this process by hand, but the computer is more reliable and therefore faster and easier in the long run. Therefore, we perform the following three steps:

1 - The Rational Polytope. We use Mathematica to generate the rational polytope, and to transform the rational polytope so that it lives in $\mathbb{R}^{3}$ (see Appendix 2 for the code). We are careful to retain the same integer lattice.

2 - The Newton Polytope. To obtain the Newton polytope, we use the program

Qhull from the Geometry Center. Qhull is designed to enumerate extremal points of a polytope, but it generally sacrifices accuracy for speed. We use a zero-tolerance 
setting so that we get integral points back. We also set Qhull to give us output in Mathematica form. There is a Mathematica procedure which would enumerate the vertices, but it is too inefficient.

3 - Desingularization. We feed the coordinates of the Newton polytope to a Mathematica program (see Appendix 3 for code) which returns the intersection graph of curves from the desingularization.

\section{$1.7 \quad$ Forming Elliptic Fibrations}

1.7.0.1 Definition. An elliptic fibration is a regular map $\pi: S \rightarrow B$ from our surface $S$ to some base curve $B$, such that the general fibre $\pi^{-1}(b)$ is an elliptic curve.

Because our surfaces are K3 (so that $H^{1}\left(\mathcal{O}_{S}\right)=0$ and thus $g_{B}=0$ ), the base curve is isomorphic to $\mathbb{P}^{1}$. In an elliptic fibration, most fibres will be irreducible. There may also be finitely many reducible fibres; this is where the Kodaira Classification of

Fibres enters. One can check, using the adjunction formula $g=\frac{F^{2}-F \cdot K}{2}+1=\frac{F^{2}}{2}+1$, that each of these reducible fibres has arithmetic genus 1. Thus, we'll be looking for subgraphs in the output of the Mathematica program which are isomorphic to graphs of fibres from Table 1.2 (very few of the curves in the graphs which we obtain from the surfaces have genus 1 ). Notice that because we can only have $A_{n}$ singularities, we are limited to fibres which are irreducible or which correspond to Extended Dynkin Diagrams.

We want to partition each output graph into collections of subgraphs corresponding to fibres, sections, and multisections. Our partitions are subject to some con- 
straints:

Constraint 1. Distinct fibres do not intersect.

Constraint 2. A section intersects each fibre with multiplicity one. A multisection ( $n$-section) intersects each fibre with multiplicity $n$.

Constraint 3. We only consider elliptic fibrations with a finite number of sections; under this condition, all sections are torsion sections and are thus disjoint [Miranda, Lemma, p.72].

Constraint 4. The remaining aspect of forming fibrations is this: if we decompose the graph into subgraphs such that one of these subgraphs corresponds to an Extended Dynkin Diagram with a curve deleted, we may add that curve to complete the fibre.

If we wish to do this, we must show that this is the only possible completion of the fibration (which is usually trivial). We may not complete the fibre in any way which would induce additional intersections between the original curves on the graph. Of course, added curves may intersect sections and multisections as well.

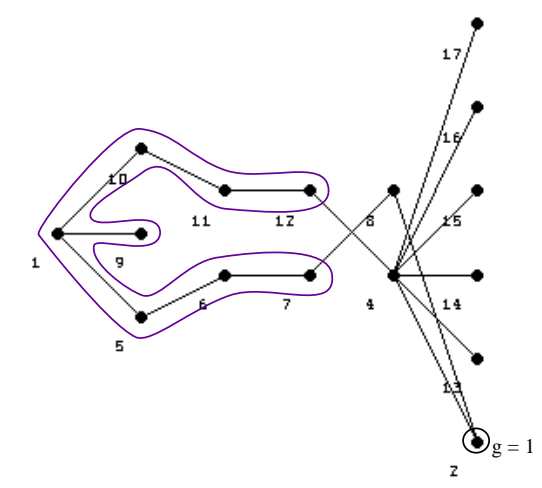

Figure 1.3: Number 26 - Step 1

1.7.0.2 Example: see Figure 1.3. 
We have chosen to view curve 2 as a fibre, so that curves 4 and 8 are sections and we have curves 1 , 5-7, 9-12 forming an $\tilde{E}_{7}$. We must complete curves 13-17 into a fibre or fibres. We know that each of these must be in a different reducible fibre because a section intersects each fibre with multiplicity one, and section 4 intersects each of these curves with multiplicity at least one. The only fibres which conform to these constraints are $\tilde{A}_{n}$; each component is of multiplicity one. In fact, for reasons which will be explained soon, we can only choose $\tilde{A}_{1}$ because otherwise we will generate a lattice with a rank larger than $\rho$. Now our fibration looks like Figure 1.4.

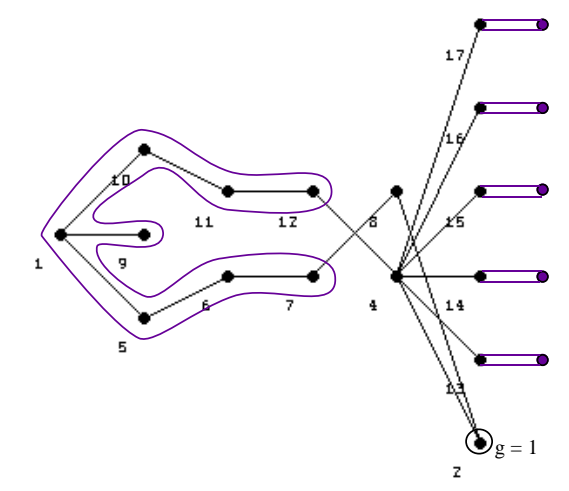

Figure 1.4: Number 26 - Step 2

It is still not complete, because section 8 must intersect each fibre once, and it currently does not intersect any of the five $\tilde{A}_{1}$. This is not a contradiction; it must intersect each of the added curves (see Figure 1.5).

\subsubsection{The Mordell-Weil group of sections}

We may view a section of the fibration $\pi: S \rightarrow \mathbb{P}^{1}$ in two ways: as a regular map $s: \mathbb{P}^{1} \rightarrow S$, or as a curve in $S$ defined by $\left\{p \in S \mid p=s(x), x \in \mathbb{P}^{1}\right\}$. We will view a section as a curve. A section crosses each fibre once, so if we restrict all the sections to the generic fibre, we can consider the rational points we obtain as a group, 


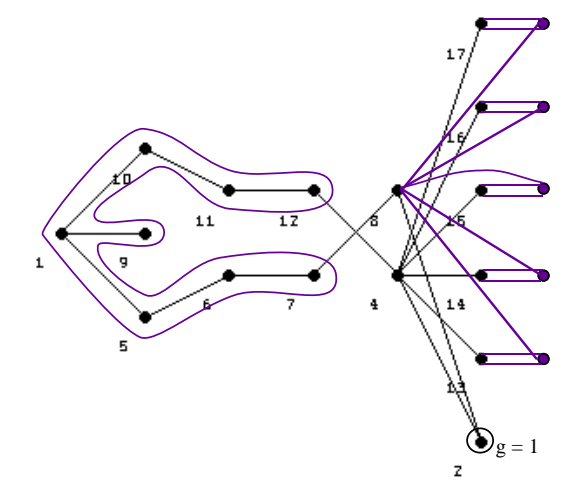

Figure 1.5: Number 26 - Final Step

using the group law for elliptic curves. If we take the closure of these points to form sections again, we can see this group as formed by the sections themselves (not just the points). We call this the Mordell-Weil group of sections (or $M W$ ); if there exists at least one singular fibre, then $M W$ is a finitely-generated abelian group [Miranda, p. 69].

\subsubsection{The Shioda-Tate Formula}

The Shioda-Tate formula is useful for analyzing possible elliptic fibrations.

1.7.2.1 Lemma [Shioda, Corollary 1.5]. Let $f: S \rightarrow B$ b an elliptic fibration of a nonsingular model of $S$, and let $\rho$ be the rank of $\operatorname{Pic}(S)$. Then

$$
\rho=2+\sum_{F_{i}}\left(\left(\sharp \text { components in } F_{i}\right)-1\right)+r k(M W),
$$

where $F_{i}$ ranges over all fibres (note that irreducible fibres will not contribute to the sum). The number "2" corresponds to the contribution from a section an irreducible fibre.

Most of the time we will find a fibration which shows that $r k(M W)=0$, i.e. $M W$ is finite. The example of number 26, above, shows this: we have a general fibre, a 
section which intersects it, a reducible fibre with 8 components, and 5 reducible fibres with 2 components each. We are given that the rank is $\rho=14$. Thus, $14=$ $2+7+5 \cdot 1+r k(M W)=14+r k(M W)$ so $r k(M W)=0$. The Shioda-Tate formula

is the reason we could only choose fibres that are $\tilde{A}_{1}$; otherwise the right-hand-side of the equation would have been larger, and $M W$ cannot have negative rank. When we have shown that $r k(M W)=0$, we have also shown that our fibration generates a finite-index subgroup of $\operatorname{Pic}(S)$ because the fibration generates a subgroup of rank $\rho$.

\subsubsection{Bounds on $|M W|$}

If we know that $M W$ is finite, we have additional information which gives us an upper and lower bound on $|M W|=$ the number of sections. Our lower bound is the number of sections we have exhibited in the fibration. The upper bound is given by the gcd of the orders of $G_{F_{i}}$, because $M W$ embeds in the discriminant group of each fibre [Miranda, p.70].

Furthermore, we have

\subsubsection{Lemma.}

$$
|M W|^{2} \operatorname{disc}(\operatorname{Pic}(S))=\prod_{F_{i}} \operatorname{disc}\left(F_{i}\right) .
$$

This follows from [Shioda, Corollary1.7], which states that

$$
\frac{\operatorname{disc}(\operatorname{Pic}(S))}{\left|\operatorname{Pic}_{\text {tors }}(S)\right|^{2}}=\frac{\operatorname{disc}\left(\left(s_{i j}\right)\right) \cdot \prod_{F_{i}} \operatorname{disc}\left(F_{i}\right)}{|M W|^{2}}
$$

$\left(\left(s_{i j}\right)\right)$ is the incidence matrix of non-torsion sections; we have none. Also, $\operatorname{Pic}_{t o r s}(S)$ is trivial because K3s are simply-connected.

Often the bounds and the use of this formula will be enough to tell us that $M W$ is 
trivial (at which point we are done, as we have shown that the index in $\operatorname{Pic}(S)=1$ ).

The next section covers the cases for which we need additional techniques.

\subsection{Computing $\operatorname{Pic}(S)$ : Specific Techniques (with an example for each)}

\subsection{1 "Obvious" Elliptic Fibrations}

\section{An Elliptic Fibration with a Genus 1 Curve}

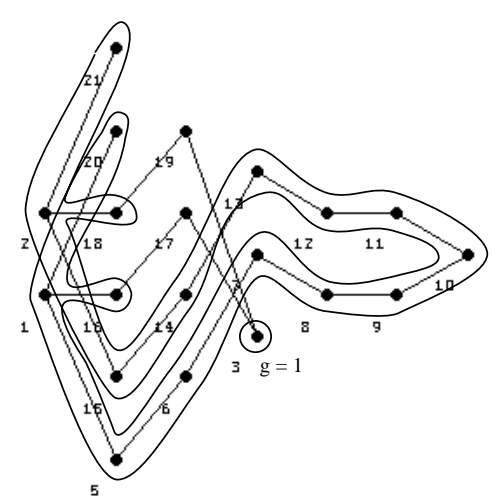

Figure 1.6: Number 65 - First Fibration

Figure 1.6 is number 65. From the output of the Mathematica desingularization program we know that curve 3 has genus 1 . It is irreducible, so we may consider it to be a fibre and then curves 17 and 19 must be sections. The remaining curves form a $\tilde{D}_{16}$. Note that curves 16 and 18 have multiplicity one, so that it makes sense for them to intersect the sections 17 and 197. We examine the Shioda-Tate formula: we know that $\rho=18$. We have a general fibre (curve 3) which intersects a section, and a 17-component reducible fibre. $2+16=18$ so the Shioda-Tate formula is satisfied with $r k(M W)=0$. We have exhibited two sections, so there are at least two sections; $G_{D_{16}}=\mathbb{Z}_{2} \oplus \mathbb{Z}_{2}$, so there are at most four sections. Now we will apply the second 
formula to get more information. $|M W|^{2} \operatorname{disc}(\operatorname{Pic}(S))=4$ and $|M W|^{2} \in\{4,9,16\}$. In order for $\operatorname{disc}(\operatorname{Pic}(S))$ to be an integer, the only possibility is that $|M W|^{2}=4$, indicating that there are exactly two sections and that $\operatorname{disc}(\operatorname{Pic}(S))=1$. We notice immediately that therefore $\operatorname{Pic}(S) \neq D_{16} \perp U$ because that lattice has discriminant 4. In a few subsections, we will see what to do in such cases.

\section{An Example of an Elliptic Fibration with no Genus 1 Curve}

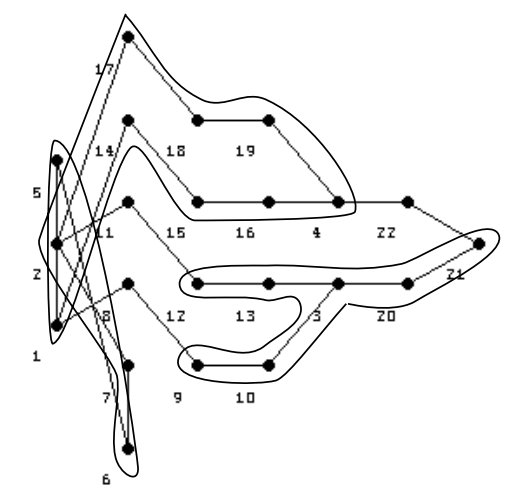

Figure 1.7: Number 52

In number 52 (see Figure 1.7), there is no elliptic curve, only -2-curves. We can see an $\tilde{E}_{6}$ in curves $3,9,10,12,13,20,21$. Then, curves 8,11 , and 22 must be sections as curves 9, 12, and 21 intersect with multiplicity one. The remaining curves form an $\tilde{A}_{11}$. Once again, we examine the Shioda-Tate formula. We have at least one fibre and at least one section, and two degenerate fibres with 7 and 12 components respectively. $\rho=19 ; 2+6+11=19$ so we know that $r k(M W)=0$. There are at least three sections (we have exhibited three) and at most three sections $\left(G_{E_{6}}=\mathbb{Z}_{3}, G_{A_{11}}=\mathbb{Z}_{12}\right)$, so $|M W|=3$. Using the other formula, we see that $9 \cdot \operatorname{disc}(\operatorname{Pic}(S))=36$. Therefore, $\operatorname{disc}(\operatorname{Pic}(S))=4$. Again, we have not yet determined $\operatorname{Pic}(S)$ but we will see what to do soon. 


\section{What If We Find More Than One Fibration? Notions of Isomorphism Revisited}

It happens frequently that we find more than one fibration. A good example is number 65: in Figure 1.6, we see an elliptic fibration for number 65. See Figure 1.8 for another fibration.

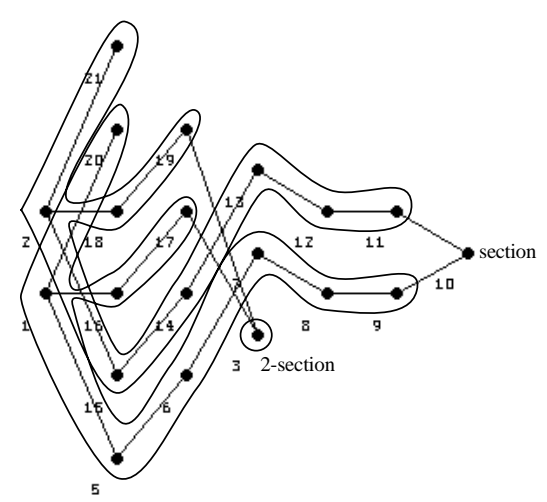

Figure 1.8: Number 65 - Second Fibration

In this fibration, we have chosen to notice that we have two copies of $\tilde{E}_{8}$; we view curve 3 as a 2 -section and curve 10 as a section. Here Shioda-Tate reads $18=2+16+r k(M W)$, indicating that $r k(M W)=0$. We have at least one section (curve 10) and at most one section $\left(G_{E_{8}}\right.$ is trivial); the formula $|M W|^{2} \operatorname{disc}(\operatorname{Pic}(S))=$ $\prod_{F_{i}} \operatorname{disc}\left(F_{i}\right)$ reads as $1 \cdot \operatorname{disc}(\operatorname{Pic}(S))=1$. This matches the discriminant of our fibration, so we are done and $\operatorname{Pic}(S)=E_{8} \perp E_{8} \perp U$. From the previous fibration (Figure 1.6), we concluded that $\operatorname{disc}(\operatorname{Pic}(S))=1$ and from Figure 1.8 we concluded the same thing. In cases where there seems to be a discrepancy, we reconcile it using the isomorphism relations on the forms corresponding to the two lattices. 


\subsection{2 $T_{p, q, r}$ Fibrations}

Sometimes we will not find any satisfactory fibrations using the Dynkin diagrams, but will instead find a large configuration of curves in the shape of a $\mathbf{T}$. We denote these lattices by $T_{p, q, r}$ where $p, q$, and $r$ are the lengths of the three legs, counting the central vertex each time. The discriminant of a $T_{p, q, r}$ is $p q r-p q-p r-q r$ and its rank is $p+q+r-2$. Some of these lattices are isomorphic to Dynkin diagrams; for example, $T_{2,3,7}$ is $E_{8} \perp U$. These lattices are well-known; a good source of detailed in-depth information on them is [Brieskorn]. There is some additional information in Chapter 2 and Appendix 1.

1.8.2.1 Example. This is number 4, which is one of Arnold's singularities.

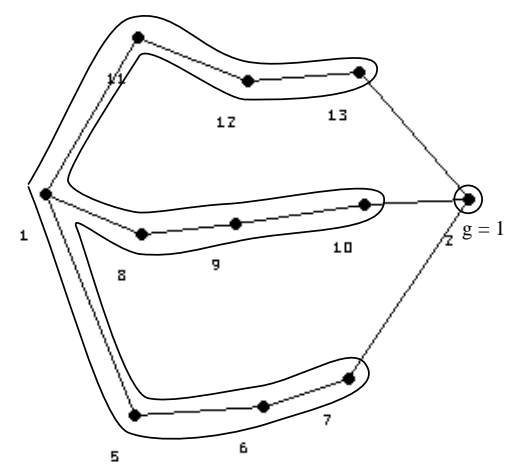

Figure 1.9: Number 4

There is no Dynkin diagram (or combination thereof) which gives us a good fibration, but $T_{4,4,4}$ is exactly what we need. For Arnold's singularities, this is sufficient, but for other cases we will need to use techniques from sections 1.8 .3 and 1.8.5 to show that the $T_{p, q, r}$ has index 1 in $\operatorname{Pic}(S)$. 


\subsubsection{Intermediate Lattice Calculations}

This technique deals with the problems we had with numbers 65 and 52 above. In general, we use an intermediate lattice calculation when we have exhibited more than one section, especially when the number of exhibited sections divides $\prod_{F_{i}} \operatorname{disc}\left(F_{i}\right)$. First, we recall that because we've found a lattice of the correct rank, then it must embed in $\operatorname{Pic}(S)$ with finite index. If $L$ is the lattice corresponding to our fibration,

then $L \subseteq \operatorname{Pic}(S) \subseteq L^{\star}$. Additionally, there is a 1-1 correspondence between the possible "intermediate lattices" $M$ and $q$-isotropic subgroups of the discriminant group $G_{L}$ [Nikulin, 1.4.1(a)]. In fact, there is a constructive method for listing the different possibilities for $\operatorname{Pic}(S)$ via a formula of Nikulin:

1.8.3.1 Theorem, [Nikulin, 1.4.1(b)]. $q_{M}=\left(q_{L} \mid H^{\perp}\right) / H$, where $H$ is a $q_{L^{-}}$ isotropic subgroup of $G_{L}$.

Let us interpret this statement via an example.

1.8.3.2 Example: Number 26.

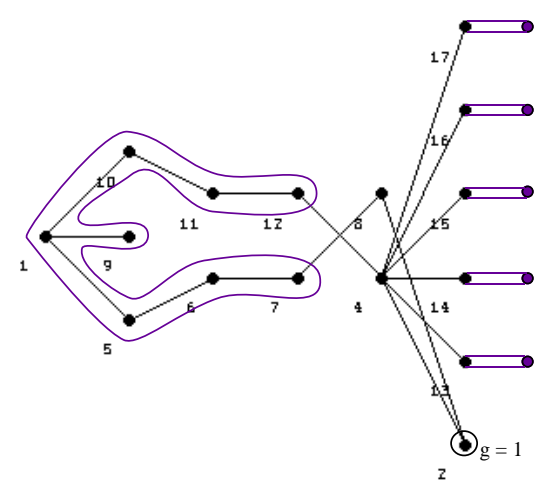

Figure 1.10: Number 26

Step 1: Find all $q_{L}$-isotropic subgroups of $G_{L}$. A subgroup is isotropic if every element of the subgroup has value $q_{L}$-value $0(\bmod 2 \mathbb{Z})$. 
For number 26 , we have $\tilde{E}_{7}+5 \tilde{A}_{1}$. Each of these degenerate fibres has discriminant group $\mathbb{Z}_{2}$, so we're beginning with $\left(\mathbb{Z}_{2}\right)^{6}$. The form corresponding to $E_{7}$ is $w_{2,1}^{1}$ and the value on the generator is $1 / 2$. The form corresponding to $A_{1}$ is $w_{2,1}^{-1}$ and the value on the generator is $-1 / 2$. These forms are independent, so in evaluating them on $\left(\mathbb{Z}_{2}\right)^{6}$ we can just add the values on the components. Immediate examples of $q_{L^{-}}$ isotropic subgroups are those generated by $(1,0,0,0,0,1),(1,0,0,0,1,0),(1,0,0,1,0,0)$, $(1,0,1,0,0,0)$, and $(1,1,0,0,0,0)$. While this seems like a lot of subgroups already, we also have

1.8.3.3 Theorem, [Nikulin, 1.4.2] Two intermediate lattices $M_{1}, M_{2}$ are isomorphic if and only if the corresponding $q_{L}$-isotropic subgroups $H_{1}, H_{2}$ of $G_{L}$ are conjugate under an automorphism of $L$.

Remark. This corresponds, for example, to the case where we have several copies of some Dynkin diagram and we permute them, or equivalently to several copies of a form where we permute the corresponding coordinates.

So in our example, all five of the $q_{L}$-isotropic subgroups can be represented without redundancy by $(1,1,0,0,0,0)$. In similar fashion, we have two other distinct isotropic subgroups represented by $(0,0,1,1,1,1)$ and $(1,1,1,1,1,1)$.

Step 2: Determine $H^{\perp}$.

To simplify the example, we will only do this for $H=(0,0,1,1,1,1)$. Up to permutation of the entries, we really only have 5 elements to deal with: $(0,0,0,0,0,0),(0,0,0,0,0,1)$, $(0,0,0,0,1,1),(0,0,0,1,1,1),(0,0,1,1,1,1)$. We will suppress the first 2 entries as they are always 0 . Now we determine which of these are perpendicular to $H=\langle(1,1,1,1)\rangle$ with respect to the quadratic form. This is true for an element $a$ when $q(a)-$ $q(a+(1,1,1,1))=0$.

So respectively, for these 5 types of elements, we have the data in Table 1.5. 


\begin{tabular}{|l|l|l|}
\hline$a$ & $a+(1,1,1,1)$ & $q(a)-q(a+(1,1,1,1))$ \\
\hline$(0,0,0,0)$ & $(1,1,1,1)$ & $0-0=0$ \\
\hline$(0,0,0,1)$ & $(1,1,1,0)$ & $(-1 / 2)-(-3 / 2)=1$ \\
\hline$(0,0,1,1)$ & $(1,1,0,0)$ & $(-1)-(-1)=0$ \\
\hline$(0,1,1,1)$ & $(1,0,0,0)$ & $(-3 / 2)-(-1 / 2)=-1$ \\
\hline$(1,1,1,1)$ & $(0,0,0,0)$ & $0-0=0$ \\
\hline
\end{tabular}

Table 1.5: Calculation of $H^{\perp}$ for Number 26

Step 3: List all elements in $H^{\perp}$ and their values on $q_{L}$. Group them by conjugacy class in order to mod out by $H$. Using this list of values, determine the form of the intermediate lattice corresponding to $H$.

Table 1.6 lists all elements of $H=\langle(1,1,1,1)\rangle$ by conjugacy class, and their $q_{L}$-values.

\begin{tabular}{|c|c|c|}
\hline elt & elt $+(1,1,1,1)$ & $q_{L}$ value \\
\hline$(0,0,0,0)$ & $(1,1,1,1)$ & 0 \\
\hline$(0,0,1,1)$ & $(1,1,0,0)$ & -1 \\
\hline$(0,1,0,1)$ & $(1,0,1,0)$ & -1 \\
\hline$(0,1,1,0)$ & $(1,0,0,1)$ & -1 \\
\hline
\end{tabular}

Table 1.6: Values of the form $q_{M}$ corresponding to $H$ for Number 26

This data corresponds to the form $v$ (one can check this in the table in Appendix 1). We must also retain the original form on the first two copies of $\mathbb{Z}_{2}\left(w_{2,1}^{-1} \perp w_{2,1}^{1}\right)$ because they weren't involved in the calculation; they correspond to the zeros we suppressed above.

In the example of number 26 , we only computed $q_{M}$ for one of the three distinct $q_{L}$-isotropic subgroups. Number 26 is very illustrative in that $q_{M^{\prime}}$ and $q_{M^{\prime \prime}}$ (corre- 
sponding to the other two $q_{L}$-isotropic subgroups $H^{\prime}$ and $H^{\prime \prime}$ ) are not isomorphic to $v \perp w_{2,1}^{-1} \perp w_{2,1}^{1}$. (Respectively, they are $\left(w_{2,1}^{-1}\right)^{4}$ and $u \perp v$.) This creates another problem: which one is correct? We need to look on the graph for other fibrations which confirm that one of these choices is correct and that the others are not possible. To see how this works, examine Section 26 in Chapter 3.

\subsubsection{Methods for Fibrations Without Sections}

Sometimes we'll only be able to find a fibration which has only multisections, and no sections.

1.8.4.1 Definition. A Jacobian fibration is one which exhibits a section. We construct it by taking the Jacobian variety of the generic fibre and considering this as the generic fibre of some elliptic surface.

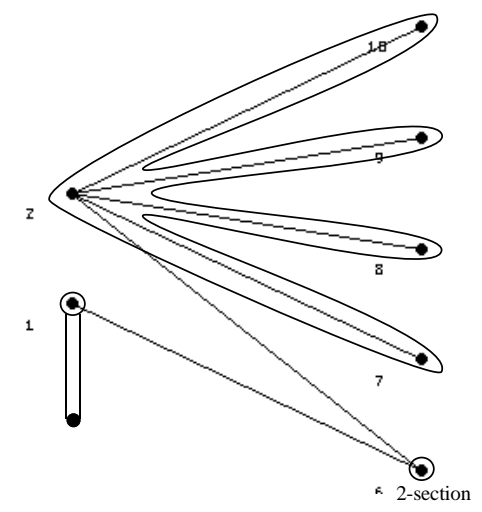

Figure 1.11: Fibration for Number 19

1.8.4.2 Example. In number 19 (Figure 1.11) we immediately see that curves 2, 7-10 form a $\tilde{D}_{4}$. Thus curve 6 is a 2 -section and we can complete curve 1 to form an $\tilde{A}_{1}$.

By definition, this is a non-Jacobian fibration. 
1.8.4.3 Lemma, [Enriques I, Proposition 5.2.5]. The fibres of $S$ are the same as those of $\mathbf{J}(S)$.

For our example, number 19, there is no fibration in which one can exhibit a section and satisfy Shioda-Tate with $r k(M W)=0$. However, the Jacobian fibration associated to Figure 1.11 satisfies Shioda-Tate with $r k(M W)=0 ; \rho=7$ so we have $7=2$ $+4+1$.

In other words, a non-Jacobian fibration gives us information about $\operatorname{Pic}(\mathbf{J}(S))$ and what we now need to know is the relationship between $\operatorname{Pic}(S)$ and $\operatorname{Pic}(\mathbf{J}(S))$.

We will show in Chapter 4 that there exists a map

$$
\phi: \operatorname{Pic}(S) \rightarrow \operatorname{Pic}(\mathbf{J}(S))
$$

of finite index $n$, where $n$ is the index of multisections, i.e. if we have 3 -sections in our fibration, then $n=3$. This completely determines $\operatorname{Pic}(S)$, though we do need the help of other techniques to complete the calculation. Practically speaking, this usually says that we can just determine $\operatorname{Pic}(S)$ from the matrix returned by the computer. This result was used to calculate $\operatorname{Pic}(S)$ in the nine cases for which we could not exhibit a section.

\subsubsection{Calculating $\operatorname{Pic}(S)$ from the Matrix}

When all else fails, for example when we cannot find a fibration at all, or when we cannot show that $M W$ is finite, we can try to calculate the quadratic form for $\operatorname{Pic}(S)$ directly from the matrix.

There are $\rho+3$ vertices on each graph, so the determinant of the incidence matrix is always zero. To determine the form, one must first remove the three extraneous rows/columns from the matrix. We may determine the appropriate discriminant 
by computing all the $\rho \times \rho$ minors and finding the minimum non-zero value; then, we assure that the vectors we remove are integral linear combinations of the remaining vectors. These operations are easy in Mathematica, using the Minors and LinearSolve commands.

Now, how do we know that this lattice sits primitively in $\operatorname{Pic}(S)$ ? We know that a fibration gives us something of finite index in $\operatorname{Pic}(S)$, so if the discriminant of the matrix is square-free, then the index is 1 and we need merely determine which (again, usually unique) form the matrix corresponds to. In every case where we showed $M W$ to be infinite, the discriminant of the matrix was square-free. In the case where we use the Mukai method, then we know the discriminant of $\operatorname{Pic}(S)$ - it matches that of the matrix, so we are again able to proceed.

Note. The matrices and graphs have dimension $(\rho+3) \times(\rho+3)$, and rank $\rho$, so to determine the discriminant of such a matrix we must find the minimum value of the determinants of the $\rho \times \rho$ minors.

It should be mentioned that there is no general algorithm for determining the quadratic form which corresponds to a matrix. One must decide based on the values of the form on its generators and on the relations between these generators.

Remember, the discriminant quadratic form $q$ is a map defined on the discriminant group $G_{L}$,

$$
q: L^{\star} / L \rightarrow \mathbb{Q} / 2 \mathbb{Z}
$$

To evaluate the form on an element $v \in G_{L}$ using its matrix $A_{q}$, we evaluate ${ }^{t} v \cdot A_{q} \cdot v$. Of course, we wonder how to see the basis vectors $e_{i}^{\star}$ of $G_{L}$ ! Notice that for $v$ to be an element of $L^{\star}, v \in L \otimes \mathbb{Q}$ and $\langle v, \ell\rangle$ for all $\ell \in L$. The columns $c_{j}$ of $A_{q}^{-1}$ satisfy the condition

$$
{ }^{t} c_{j} \cdot A_{q} \cdot e_{i} \in \mathbb{Z}
$$


and in fact give only the values $\{0,1\}$. Thus, their images give the basis for $\mathbb{Z}^{\rho}$ and so we may legally call the $c_{j} e_{i}^{\star}$ instead. So, the set of values of

$$
{ }^{t}\left(e_{i}^{\star}\right) \cdot A_{q} \cdot e_{i}^{\star}
$$

are the values of $q$ on the basis vectors of $L^{\star}$. And, in fact, we can now see that any element $v \in G_{L}$ can be expressed as a linear combination of the column vectors of $A_{q}^{-1}$, so we may intuit the relations between the generators from their values. Finally, modding out by $L$ on the left-hand-side of the map is equivalent to modding out by $2 \mathbb{Z}$ on the right-hand-side.

\subsubsection{Generalizations and Other Questions}

There are a few questions left unanswered by our calculations.

1.8.6.1 Question: We notice that not all families have mirrors on the list. Where are those mirrors?

Kreuzer and Skarke have done work which gives us an algorithm to find all 3dimensional reflexive polytopes, and therefore all K3 surfaces which can be realized as toric hypersurfaces. Many of the mirrors may be on this list.

1.8.6.2 Question: We notice that there are some distinct families which have the same $\operatorname{Pic}(S)$. What's going on there? Are they isomorphic, or is something different happening, and if so, what?

Miles Reid has a conjecture which does not explain this phenomenon, but perhaps will lead to some enlightenment. Chapter 2 is devoted to a discussion of this conjecture. 


\section{CHAPTER II}

\section{Miles Reid's Conjecture}

\subsection{Statement of the Conjecture}

Consider the quotient map

$$
\bar{f}: \mathbb{P}^{3} \rightarrow \mathbb{P}\left(q_{0}, q_{1}, q_{2}, q_{3}\right)=\mathbb{P}^{3} /\left(\mu_{q_{0}} \times \mu_{q_{1}} \times \mu_{q_{2}} \times \mu_{q_{3}}\right)
$$

which defines weighted projective space. Denote the coordinates of $\mathbb{P}^{3}$ as $x_{0}, x_{1}, x_{2}, x_{3}$; then $\bar{f}$ sends $x_{j} \mapsto \bar{x}_{j}=x_{j}^{q_{j}}$. The restriction of this map to the hypersurface $S$ will be called $f$. Even though $\mathcal{O}_{S}(1)$ is not necessarily locally free, it does correspond to some Weil divisor $D . D$ is $\mathbb{Q}$-Cartier, so the self-intersection of $D$ is well-defined as $D^{2}=\frac{(n D)^{2}}{n^{2}}$, where $n$ is the smallest multiple of $D$ which is Cartier. We will calculate $D^{2}$ more concretely: first notice that self-intersection changes by the degree of $f$ when we pull back, so $f^{*}(D)^{2}=D^{2} \cdot \prod_{j} q_{j}$. Then notice that $f^{*}(D)^{2}=\left(\mathcal{O}_{f^{*}(S)}(1)\right)^{2}$, which is the degree of $f^{*}(S)$. If we write the variables in $\mathbb{P}\left(q_{0}, q_{1}, q_{2}, q_{3}\right)$ as $x_{j}^{q_{j}}$ instead of as $\bar{x}_{j}$, we can see that $\operatorname{deg}_{\mathbb{P}^{3}}\left(f^{*}(S)\right)=\operatorname{deg}_{P\left(q_{0}, q_{1}, q_{2}, q_{3}\right)}(S)$. Finally, we combine these facts to see that

$$
D^{2} \cdot \prod_{j} q_{j}=f^{*}(D)^{2}=\left(\mathcal{O}_{f^{*}(S)}(1)\right)^{2}=\operatorname{deg}_{P\left(q_{0}, q_{1}, q_{2}, q_{3}\right)}(S)=d .
$$


We then have that

$$
D^{2}=\frac{d}{\prod_{j} q_{j}}
$$

Now we will desingularize $S$; it has cyclic quotient singularities $A_{p_{j}}$. The desingularization map is $\pi: \tilde{S} \rightarrow S$. Denote the preimage of $D$ as $\pi^{-1}(D)=R+D^{\prime}$, where $D^{\prime}$ is the portion of $\pi^{-1}(D)$ which contain exceptional curves and $R=\pi^{*}(D)$ is the portion which does not contain any exceptional curves. Denote by $\overline{A_{p_{j}}}$ the minimal resolution of each $A_{p_{j}}$.

2.1.0.3 Conjecture [Reid]. $R \cdot \overline{A_{p_{j}}}=1$; in fact, this "intersection" is in the $i_{j}$-th component. Furthermore, $R$ is linearly independent from the exceptional curves of the $A_{p_{j}}$, and together these curves generate $\operatorname{Pic}(S)$.

We may also express $D^{2}$ in terms of the $A_{p_{j}}$, as

$$
D^{2}=R^{2}+\sum_{j} \frac{i_{j}\left(p_{j}+1-i_{j}\right)}{p_{j}+1}[\text { Reid }] .
$$

Reid refers to this set of information as numerical data. This data may be visualized in the form of a graph $M_{\vec{p}, \vec{\imath}, R^{2}}$, which will be discussed in section 2.2 .

A more general form of 2.1.0.3 appears in [Reid]; we verify in section 2.3 that using Reid's conjecture to compute $\operatorname{Pic}(S)$ for the 95 families gives the same results as the methods described in Chapter 1 . Notice that the conjecture depends on $\rho=1$ before desingularization, so that one must be cautious in using the conjecture for a wider class of surfaces.

\subsection{The $M_{\vec{p}, \vec{l}, k}$ Lattices}

2.2.0.4 Definition. Let $\vec{p}=\left(p_{1}, \ldots, p_{n}\right)$ be an $n$-tuple of positive integers, ordered from least to greatest. Let $\vec{i}=\left(i_{1}, \ldots, i_{n}\right)$ be an $n$-tuple of integers such that $i_{j} \leq$ 
$\left\lceil\frac{p_{j}}{2}\right\rceil$. Let $k \geq-4$ be an even integer. Then $M_{\vec{p}, \vec{l}, k}$ is the lattice defined by the incidence matrix of the following graph:

Begin with a central vertex $c$ with self-intersection $k$. For each $j$, adjoin to this vertex a Dynkin diagram of type $A_{p_{j}}$ by adding an edge between $c$ and vertex $i_{j}$ of the Dynkin diagram.

Dolgachev has calculated the discriminant of a $M_{\vec{p}, \vec{l}, k}$; it is

$$
\operatorname{disc}\left(M_{\vec{p}, \vec{\iota}, k}\right)=-(-1)^{\sum_{j=1}^{n} p_{j}}\left(p_{1}+1\right) \ldots\left(p_{n}+1\right)\left(k+\sum_{j=1}^{n} \frac{i_{j}\left(p_{j}+1-i_{j}\right)}{p_{j}+1}\right) .
$$

When $k=-2, n=3, i_{j}=1$, we have a $T_{p, q, r}$ lattice.

Note that there is no algorithm for computing the associated quadratic form (even for the $T_{p, q, r}-$ see [Brieskorn]). Therefore, we cannot determine which $M_{\vec{p}, \vec{\imath}, k}$ have primitive embeddings into $\left(E_{8}\right)^{2} \perp(U)^{3}$, as we do not know the number of generators of the form in general [Nikulin, 1.12.3].

\subsubsection{Questions of Isomorphism}

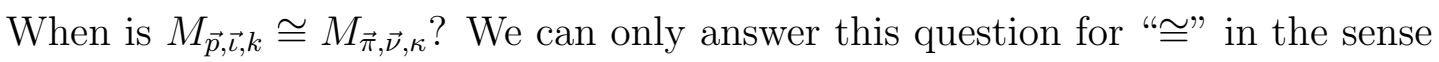
of graph theory, not of lattice theory. In terms of lattices, we have many examples where $M_{\vec{p}, \vec{l}, k} \cong M_{\vec{\pi}, \vec{\nu}, \kappa}$ but the graphs are not isomorphic - see, in section 2.3, almost any pair where $\operatorname{Pic}(S)=\operatorname{Pic}\left(S^{\prime}\right)$. This is true also for the $T_{p, q, r}$ lattices; $T_{2,7,7} \cong$ $T_{3,3,10}$ [Brieskorn] but the graphs are not isomorphic. One minor illumination: isomorphism of lattices is determined when the matrices have the relation $M=$ $P M P^{t}$, for $P$ a composition of elementary row and column operations. We may restrict $P$ to a composition of elementary operations of the third kind $\left(r_{i} \rightarrow \lambda r_{j}+\right.$ $\left.r_{i}, \lambda \in \mathbb{Z}\right)$ because the those of the first and second kinds are trivial over the integral symmetric bilinear forms. 
2.2.1.1 Lemma. $M_{\vec{p}, \vec{l}, k} \cong M_{\vec{\pi}, \vec{\nu}, \kappa}$ as labeled graphs if and only if the following hold:

- $k=-2$

- $n$ and $n^{\prime}$ are each less than or equal to 3

- at most one $i_{j} \neq 1$.

Under these conditions, we have the following isomorphism relations:

- for $p_{3} \geq 3, M_{\left(p_{1}, p_{2}, p_{3}\right),(1,1, i),-2} \cong M_{\left(i-1, p_{3}-i, p_{1}+p_{2}+1\right),\left(1,1, p_{1}+1\right),-2}$

$\cong M_{\left(i-1, p_{3}-i, p_{1}+p_{2}+1\right),\left(1,1, p_{2}+1\right),-2}$

- $M_{\left(p_{1}, p_{2}, p_{3}\right),(1,1,1),-2} \cong M_{\left(p_{1}-1, p_{2}+p_{3}+1\right),\left(1, p_{2}+1\right),-2} \cong M_{\left(p_{1}-1, p_{2}+p_{3}+1\right),\left(1, p_{3}+1\right),-2}$

$\cong M_{\left(p_{2}-1, p_{1}+p_{3}+1\right),\left(1, p_{1}+1\right),-2} \cong M_{\left(p_{2}-1, p_{1}+p_{3}+1\right),\left(1, p_{3}+1\right),-2} \cong M_{\left(p_{3}-1, p_{1}+p_{2}+1\right),\left(1, p_{1}+1\right),-2}$

$\cong M_{\left(p_{3}-1, p_{1}+p_{2}+1\right),\left(1, p_{2}+1\right),-2}$

- for $p_{2} \geq 3, M_{\left(p_{1}, p_{2}\right),(1, i),-2} \cong M_{\left(p_{1}+1, i-1, p_{2}-i\right),(1,1,1),-2}$

- $M_{\left(p_{1}, p_{2}\right),(1,1),-2} \cong M_{\left(j-1, p_{1}+p_{2}-j\right),(1,1),-2}$

Proof. The key is to notice that two different $M_{\vec{p}, \vec{l}, k}$ graphs can be isomorphic only if we can move the central vertex from one spot $c$ to another $c^{\prime}$. This immediately tells us that $k=-2$. Then, if there are more than three branches, we cannot move $c$ in any way such that the branches from $c^{\prime}$ form $A_{s}$. Therefore, $n \leq 3$. Finally, we have the same problem if more than one branch from $c$ is forked.

We prove the isomorphism relations with pictures; figure 2.1 proves the third relation.

The reader may convince herself of the remaining isomorphism relations by drawing similar pictures.

\subsubsection{Signature of $M_{\vec{p}, \vec{l}, k}$}

In [Brieskorn, $\S 1.9]$ the author calculates the signature of the $T_{p, q, r}$ lattices from the formula for the discriminant. We have similar results for $M_{\vec{p}, \vec{\imath}, k}$ and will prove them in a similar fashion. To provide motivation, we re-cast the proof for the $T_{p, q, r}$ 

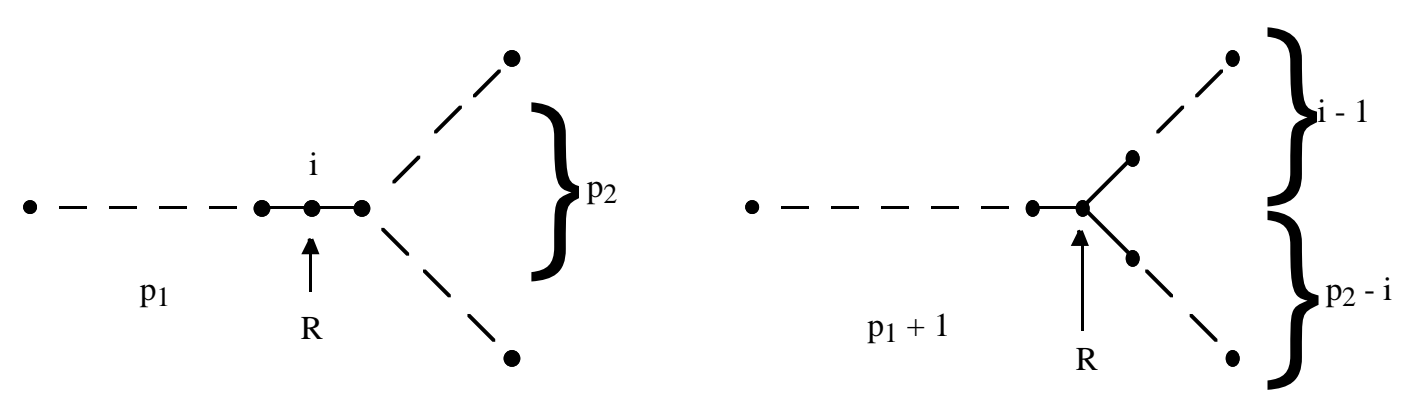

Figure 2.1: Proof of third relation in Lemma 2.2.1.1

in the notation for $M_{\vec{p}, \vec{\iota}, k}$; notice that $T_{p, q, r}=M_{(p-1, q-1, r-1),(1,1,1),-2}$. Thus,

$$
\operatorname{disc}\left(T_{p, q, r}\right)=-(-1)^{p+q+r-3} p \cdot q \cdot r \cdot\left(-2+\frac{p-1}{p}+\frac{q-1}{q}+\frac{r-1}{r}\right) .
$$

2.2.2.1 Lemma, [Brieskorn]. Denote by $\rho$ the rank of $T_{p, q, r}$. Then, the signature $\left(\rho_{-}, \rho_{+}, \rho_{0}\right)$ of $T_{p, q, r}$ is

- $(\rho-1,1,0)$ when $-2+\frac{p-1}{p}+\frac{q-1}{q}+\frac{r-1}{r}>0$

- $(\rho-1,0,1)$ when $-2+\frac{p-1}{p}+\frac{q-1}{q}+\frac{r-1}{r}=0$

- $(\rho, 0,0)$ when $-2+\frac{p-1}{p}+\frac{q-1}{q}+\frac{r-1}{r}<0$.

2.2.2.2 Proof of 2.2.2.1. First, notice that if we consider the sublattice defined by the graph $M_{(p-1, q-1, r-1),(1,1,1),-2} \backslash$ central vertex, we see that its matrix is negative definite because $M_{(p-1, q-1, r-1),(1,1,1),-2} \backslash$ central vertex $=\oplus A_{j}$. Therefore $\rho_{-} \geq \rho-1$. First, if $\operatorname{disc}\left(T_{p, q, r}\right)$ is zero, then the signature must be $(\rho-1,0,1)$ and $-2+\frac{p-1}{p}+$ $\frac{q-1}{q}+\frac{r-1}{r}=0$ as $p, q, r,-1 \neq 0$. Now, suppose $\rho$ is odd. If $\operatorname{disc}\left(T_{p, q, r}\right)$ is positive (i.e. $\left.-2+\frac{p-1}{p}+\frac{q-1}{q}+\frac{r-1}{r}>0\right)$, then $\rho_{-}$is even; as $\rho-1$ is even, then the signature must be $(\rho-1,1,0)$. If $\operatorname{disc}\left(T_{p, q, r}\right)$ is negative (i.e. $\left.-2+\frac{p-1}{p}+\frac{q-1}{q}+\frac{r-1}{r}<0\right)$, then $\rho_{-}$ 
is odd; as $\rho_{-} \geq \rho-1$ and $\rho-1$ is even, then the signature must be $(\rho, 0,0)$. Now suppose $\rho$ is even. If $\operatorname{disc}\left(T_{p, q, r}\right)$ is positive (i.e. $-2+\frac{p-1}{p}+\frac{q-1}{q}+\frac{r-1}{r}<0$ ), then $\rho_{-}$is even; as $\rho_{-} \geq \rho-1$ and $\rho-1$ is odd, then the signature must be $(\rho, 0,0)$. If $\operatorname{disc}\left(T_{p, q, r}\right)$ is negative (i.e. $-2+\frac{p-1}{p}+\frac{q-1}{q}+\frac{r-1}{r}>0$ ), then $\rho_{-}$is odd; as $\rho-1$ is odd, then the signature must be $(\rho-1,1,0)$.

2.2.2.3 Lemma. Denote by $\rho$ the rank of $M_{\vec{p}, \vec{l}, k}$. Then, the signature $\left(\rho_{-}, \rho_{+}, \rho_{0}\right)$ of $M_{\vec{p}, \vec{\imath}, k}$ is

- $(\rho-1,1,0)$ when $k+\sum_{j=1}^{n} \frac{i_{j}\left(p_{j}+1-i_{j}\right)}{p_{j}+1}>0$

- $(\rho-1,0,1)$ when $k+\sum_{j=1}^{n} \frac{i_{j}\left(p_{j}+1-i_{j}\right)}{p_{j}+1}=0$

- $(\rho, 0,0)$ when $k+\sum_{j=1}^{n} \frac{i_{j}\left(p_{j}+1-i_{j}\right)}{p_{j}+1}<0$.

2.2.2.4 Proof of 2 2.2.3. Notice that the proof of 2.2 .2 .1 does not depend on the shape of the graph being a $T_{p, q, r}$ ! It will work for any $M_{\vec{p}, \vec{\iota}, k}$ - thus, we are done.

2.2.2.5 Partial Classification of Hyperbolic $M_{\vec{p}, \vec{l}, k}$. Most $M_{\vec{p}, \vec{l}, k}$ are hyperbolic; therefore, we list those with signature other than $(\rho-1,1,0)$. For $k \geq 0$, there are only hyperbolic lattices. For $k=-2$, the following $\vec{p}, \vec{\imath}$ give $\operatorname{disc}\left(M_{\vec{p}, \vec{\imath}, k}\right)=$ 0 : $\quad(1,1,1,1),(1,1,1,1) ; \quad(2,2,2),(1,1,1) ; \quad(3,3),(2,2) ; \quad(4,4),(2,1) ; \quad(5,1),(3,1) ; \quad(5,2),(2,1)$; $(7,1),(2,1) ;(7),(4) ;(8),(3)$. For $k=-2$, the following $\vec{p}, \vec{\iota}$ give negative-definite $M_{\vec{p}, \vec{l}, k}:\left(p_{j}\right),(1) ;\left(p_{j}\right)(2) ;\left(p_{j}, p_{j^{\prime}}\right),(1,1) ;(7),(3) ;(6),(3) ;(5),(3) ;(6,1),(2,1) ;(5,1),(2,1) ;$ $(4,3),(2,1) ;(4,2),(2,1) ;(4,1),(2,1) ;(3,2),(2,1) ;(3,1),(2,1) ;(2,2,1),(1,1,1) ;(2,1,1),(1,1,1)$; $(1,1,1),(1,1,1)$. We will not classify lattices with $k=-4$ other than to say that when no $p_{j}>4$, there are at least 21 general rules and at least 51 individual cases. However, we have tested all of the 95 Families' $M_{\vec{p}, \vec{l}, k}$ which have $k=-4$ and all are hyperbolic. 


\subsection{Calculation of $\mathrm{Pic}(S)$ for the $95 \mathrm{~K} 3 \mathrm{Hypersurfaces}$ Using Reid's Conjecture}

In order to calculate $\operatorname{Pic}(S)$, we must determine first $M_{\vec{p}, \vec{\imath}, k}$ and then the quadratic form to which it corresponds. We find $\vec{p}$ from [Yonemura], which lists the cyclic quotient singularities of each hypersurface.

2.3.0.6 Fact. $k=-4+2\left(\sharp 1\right.$ 's in $\left.\left(q_{0}, q_{1}, q_{2}, q_{3}\right)\right)$.

2.3.0.7 Proof of 2.3.0.6. $k=R^{2}$ and we use Riemann-Roch on $S$ with $R$ to compute this. $h^{1}(R)=h^{2}(R)=0$ by [Reid, pf of 3.2], and $\chi\left(\mathcal{O}_{S}\right)=2$. Thus, RiemannRoch reads $h^{0}(R)=2+(1 / 2) R^{2}$, or $R^{2}=-4+2 h^{0}(R)$. Now, $h^{0}(R)=h^{0}(D)$ as $R$ is in the part of the preimage of $D$ which is unaffected by desingularization. The global sections of $D$ are degree one elements of $\mathcal{O}_{f^{*}(S)}(1)$, so $h^{0}(D)=\left(\sharp 1\right.$ 's in $\left.\left(q_{0}, q_{1}, q_{2}, q_{3}\right)\right)$. Therefore, $R^{2}=-4+2\left(\sharp 1\right.$ 's in $\left.\left(q_{0}, q_{1}, q_{2}, q_{3}\right)\right)$.

Now, we must determine $\vec{\iota}$. Riemann-Roch ([YPG]) reads

$$
h^{0}(S, D)=2+\frac{1}{2} D^{2}+\sum_{j=1}^{n} \frac{i_{j}\left(p_{j}+1-i_{j}\right)}{2\left(p_{j}+1\right)} .
$$

This of course must be an integer. We determine the possibilities for $\vec{\iota}$ using a Mathematica procedure (see Appendix 4); we are given $\vec{p}$, and computed $D^{2}$ in $(\star)$ above. The procedure tests each possibility for $\vec{\iota}$ and outputs those which are integers, as well as the discriminant of the matrix. In each case, there is only one such $\vec{\iota}$.

Given this information, we can produce the matrix via another Mathematica procedure (again, see Appendix 4). Then, we can examine the inverse of this matrix and determine the form it corresponds to (see Chapter 1, 1.8.5).

It is not necessary to actually do these computations! In each of the 95 cases, $\rho=r k\left(M_{\vec{p}, \vec{l}, k}\right)$ so that $M_{\vec{p}, \vec{l}, k}$ is a sublattice of $\operatorname{Pic}(S)$ of finite index. By comparing 
the discriminant of each $M_{\vec{p}, \vec{l}, k}$ with that of $\operatorname{Pic}(S)$ (these are listed in Table 3.1), we can determine what index $M_{\vec{p}, \vec{\imath}, k}$ has in $\operatorname{Pic}(S)$. In each case, the index is 1 , so we know that $M_{\vec{p}, \vec{\imath}, k}=\operatorname{Pic}(S)$. The following table lists $\vec{p}, \vec{\imath}, k$, and the discriminant.

Table 2.1: Forms of the $95 M_{\vec{p}, \vec{\imath}, k}$

\begin{tabular}{|r|l|l|l|c|c|}
\hline No. & Weight-Vector & \multicolumn{1}{|c|}{$\vec{\imath}$} & $k$ & Disc. \\
\hline 1 & $(1,1,1,1)$ & - & & 4 & - \\
2 & $(2,3,3,4)$ & $1,1,1,2,2,2,2$ & $1,1,1,1,1,1,1$ & -4 & 108 \\
3 & $(1,1,2,2)$ & $1,1,1$ & $1,1,1$ & 0 & 12 \\
4 & $(1,3,4,4)$ & $3,3,3$ & $1,1,1$ & -2 & 16 \\
5 & $(1,1,1,3)$ & - & & 2 & - \\
6 & $(1,2,2,5)$ & $1,1,1,1,1$ & $1,1,1,1,1$ & -2 & 16 \\
7 & $(1,1,2,4)$ & 1,1 & 1,1 & 0 & 4 \\
8 & $(1,2,3,6)$ & $1,1,2,2$ & $1,1,1,1$ & -2 & 12 \\
9 & $(1,4,5,10)$ & $1,4,4$ & $1,1,1$ & -2 & 5 \\
10 & $(1,1,4,6)$ & 1 & 1 & 0 & 1 \\
11 & $(2,3,10,15)$ & $1,1,1,2,2,4$ & $1,1,1,1,1,2$ & -4 & 12 \\
12 & $(1,2,6,9)$ & $1,1,1,2$ & $1,1,1,1$ & -2 & 4 \\
13 & $(1,3,8,12)$ & $2,2,3$ & $1,1,1$ & -2 & 3 \\
14 & $(1,6,14,21)$ & $1,2,6$ & $1,1,1$ & -2 & 1 \\
15 & $(3,3,4,5)$ & $2,2,2,2,2,3$ & $1,1,1,1,1,1$ & -4 & 81 \\
16 & $(3,6,7,8)$ & $1,2,2,2,2,6$ & $1,1,1,1,1,1$ & -4 & 27 \\
17 & $(2,3,5,5)$ & $1,4,4,4$ & $1,2,2,2$ & -4 & 25 \\
18 & $(1,2,3,3)$ & $1,2,2,2$ & $1,1,1,1$ & -2 & 27 \\
19 & $(1,2,2,3)$ & $1,1,1,1,2$ & $1,1,1,1,1$ & -2 & 32 \\
20 & $(1,6,8,9)$ & $1,2,8$ & $1,1,1$ & -2 & 3 \\
21 & $(1,1,1,2)$ & 1 & 1 & 2 & 5 \\
22 & $(1,3,5,6)$ & $2,2,5$ & $1,1,1$ & -2 & 9 \\
23 & $(2,2,3,5)$ & $1,1,1,1,1,1,4$ & $1,1,1,1,1,1,2$ & -4 & 64 \\
24 & $(1,2,4,5)$ & $1,1,1,4$ & $1,1,1,1$ & -2 & 12 \\
25 & $(1,1,3,4)$ & 3 & 1 & 0 & 3 \\
26 & $(2,4,5,9)$ & $1,1,1,1,1,8$ & $1,1,1,1,1,2$ & -4 & 16 \\
27 & $(2,3,8,11)$ & $1,1,1,10$ & $1,1,1,4$ & -4 & 4 \\
\hline & & & & \multicolumn{1}{c}{ continued on next page } \\
\hline
\end{tabular}




\begin{tabular}{|c|c|c|c|c|c|}
\hline \multicolumn{6}{|c|}{ continued from previous page } \\
\hline No. & Weight-Vector & $\vec{p}$ & $\vec{\iota}$ & $k$ & Disc. \\
\hline 28 & $(1,3,7,10)$ & 9 & 3 & -2 & 1 \\
\hline 29 & $(4,5,6,15)$ & $1,1,2,3,4,4$ & $1,1,1,1,1,1$ & -4 & 20 \\
\hline 30 & $(5,7,8,20)$ & $3,4,4,6$ & $1,2,2,1$ & -4 & 5 \\
\hline 31 & $(3,4,5,12)$ & $2,2,3,3,4$ & $1,1,1,1,2$ & -4 & 24 \\
\hline 32 & $(2,2,3,7)$ & $1,1,1,1,1,1,1,2$ & $1,1,1,1,1,1,1,1$ & -4 & 64 \\
\hline 33 & $(2,3,4,9)$ & $1,1,1,1,2,2,3$ & $1,1,1,1,1,1,1$ & -4 & 48 \\
\hline 34 & $(2,6,7,15)$ & $1,1,1,1,1,2,6$ & $1,1,1,1,1,1,1$ & -4 & 16 \\
\hline 35 & $(3,4,7,14)$ & $1,2,6,6$ & $1,1,2,2$ & -4 & 7 \\
\hline 36 & $(2,3,5,10)$ & $1,1,2,4,4$ & $1,1,1,2,2$ & -4 & 20 \\
\hline 37 & $(1,3,4,8)$ & $2,3,3$ & $1,1,1$ & -2 & 8 \\
\hline 38 & $(1,6,8,15)$ & $1,2,7$ & $1,1,1$ & -2 & 2 \\
\hline 39 & $(1,3,5,9)$ & $2,2,4$ & $1,1,1$ & -2 & 6 \\
\hline 40 & $(1,2,4,7)$ & $1,1,1,3$ & $1,1,1,1$ & -2 & 8 \\
\hline 41 & $(2,3,7,12)$ & $1,1,2,2,6$ & $1,1,1,1,3$ & -4 & 12 \\
\hline 42 & $(1,1,3,5)$ & 2 & 1 & 0 & 2 \\
\hline 43 & $(3,4,11,18)$ & $1,2,2,10$ & $1,1,1,3$ & -4 & 3 \\
\hline 44 & $(1,2,5,8)$ & $1,1,4$ & $1,1,2$ & -2 & 4 \\
\hline 45 & $(1,4,9,14)$ & 1,8 & 1,2 & -2 & 1 \\
\hline 46 & $(5,6,22,33)$ & $1,2,4,10$ & $1,1,2,2$ & -4 & 1 \\
\hline 47 & $(3,4,14,21)$ & $1,2,2,3,6$ & $1,1,1,1,2$ & -4 & 6 \\
\hline 48 & $(3,5,16,24)$ & $2,2,4,7$ & $1,1,1,3$ & -4 & 3 \\
\hline 49 & $(2,5,14,21)$ & $1,1,1,4,6$ & $1,1,1,1,3$ & -4 & 4 \\
\hline 50 & $(1,4,10,15)$ & $1,3,4$ & $1,1,1$ & -2 & 2 \\
\hline 51 & $(1,5,12,18)$ & 4,5 & 2,1 & -2 & 1 \\
\hline 52 & $(7,8,9,12)$ & $2,3,6,7$ & $1,1,3,1$ & -4 & 4 \\
\hline 53 & $(3,4,5,6)$ & $1,2,2,2,3,4$ & $1,1,1,1,1,1$ & -4 & 54 \\
\hline 54 & $(3,5,6,7)$ & $2,2,2,4,5$ & $1,1,1,2,1$ & -4 & 27 \\
\hline 55 & $(2,5,6,7)$ & $1,1,1,5,6$ & $1,1,1,1,3$ & -4 & 16 \\
\hline 56 & $(5,6,8,11)$ & $1,7,10$ & $1,3,2$ & -4 & 2 \\
\hline 57 & $(4,5,6,9)$ & $1,1,2,4,8$ & $1,1,1,1,2$ & -4 & 12 \\
\hline 58 & $(1,4,5,6)$ & $1,4,5$ & $1,1,1$ & -2 & 8 \\
\hline 59 & $(1,5,7,8)$ & 4,7 & 2,1 & -2 & 3 \\
\hline 60 & $(1,4,6,7)$ & $1,3,6$ & $1,1,1$ & -2 & 6 \\
\hline 61 & $(4,6,7,11)$ & $1,1,5,10$ & $1,1,1,3$ & -4 & 4 \\
\hline 62 & $(3,4,5,8)$ & $2,3,3,7$ & $1,1,1,3$ & -4 & 16 \\
\hline 63 & $(1,2,3,4)$ & $1,1,2,3$ & $1,1,1,1$ & -2 & 20 \\
\hline 64 & $(3,4,7,10)$ & $1,6,9$ & $1,2,3$ & -4 & 4 \\
\hline 65 & $(3,5,11,14)$ & 4,13 & 1,5 & -4 & 1 \\
\hline
\end{tabular}




\begin{tabular}{|c|c|c|c|c|c|}
\hline \multicolumn{6}{|c|}{ continued from previous page } \\
\hline No. & Weight-Vector & $\vec{p}$ & $\vec{\iota}$ & $k$ & Disc. \\
\hline 66 & $(1,1,2,3)$ & 1,2 & 1,1 & 0 & 7 \\
\hline 67 & $(2,3,7,9)$ & $1,2,2,8$ & $1,1,1,4$ & -4 & 9 \\
\hline 68 & $(3,4,10,13)$ & $1,3,12$ & $1,1,4$ & -4 & 2 \\
\hline 69 & $(2,3,4,7)$ & $1,1,1,1,2,6$ & $1,1,1,1,1,2$ & -4 & 32 \\
\hline 70 & $(2,3,5,8)$ & $1,1,4,7$ & $1,1,2,3$ & -4 & 12 \\
\hline 71 & $(1,3,4,7)$ & 3,6 & 1,2 & -2 & 5 \\
\hline 72 & $(1,2,5,7)$ & 1,6 & 1,3 & -2 & 3 \\
\hline 73 & $(7,8,10,25)$ & $1,4,6,7$ & $1,2,2,1$ & -4 & 2 \\
\hline 74 & $(4,5,7,16)$ & $3,3,4,6$ & $1,1,1,3$ & -4 & 8 \\
\hline 75 & $(2,4,5,11)$ & $1,1,1,1,1,3,4$ & $1,1,1,1,1,1,1$ & -4 & 32 \\
\hline 76 & $(2,5,6,13)$ & $1,1,1,1,4,5$ & $1,1,1,1,2,1$ & -4 & 16 \\
\hline 77 & $(1,5,7,13)$ & 4,6 & 2,1 & -2 & 2 \\
\hline 78 & $(1,4,6,11)$ & $1,3,5$ & $1,1,1$ & -2 & 4 \\
\hline 79 & $(2,5,9,16)$ & $1,1,4,8$ & $1,1,1,4$ & -4 & 4 \\
\hline 80 & $(4,5,13,22)$ & $1,4,12$ & $1,2,3$ & -4 & 1 \\
\hline 81 & $(2,3,8,13)$ & $1,1,1,2,7$ & $1,1,1,1,3$ & -4 & 8 \\
\hline 82 & $(1,3,7,11)$ & 2,6 & 1,2 & -2 & 2 \\
\hline 83 & $(4,5,18,27)$ & $1,3,4,8$ & $1,1,2,2$ & -4 & 2 \\
\hline 84 & $(5,6,7,9)$ & $2,4,5,6$ & $1,1,1,3$ & -4 & 9 \\
\hline 85 & $(2,3,4,5)$ & $1,1,1,2,3,4$ & $1,1,1,1,1,2$ & -4 & 56 \\
\hline 86 & $(4,5,7,9)$ & $3,6,8$ & $1,3,2$ & -4 & 5 \\
\hline 87 & $(1,3,4,5)$ & $2,3,4$ & $1,1,1$ & -2 & 13 \\
\hline 88 & $(2,5,9,11)$ & $1,4,10$ & $1,1,5$ & -4 & 3 \\
\hline 89 & $(1,2,3,5)$ & $1,2,4$ & $1,1,2$ & -2 & 11 \\
\hline 90 & $(4,6,7,17)$ & $1,1,3,5,6$ & $1,1,1,1,2$ & -4 & 8 \\
\hline 91 & $(5,6,8,19)$ & $1,4,5,7$ & $1,1,1,3$ & -4 & 4 \\
\hline 92 & $(3,5,11,19)$ & $2,4,10$ & $1,1,4$ & -4 & 2 \\
\hline 93 & $(3,4,10,17)$ & $1,2,3,9$ & $1,1,1,3$ & -4 & 4 \\
\hline 94 & $(3,4,5,7)$ & $2,3,4,6$ & $1,1,2,2$ & -4 & 19 \\
\hline 95 & $(2,3,5,7)$ & $1,2,4,6$ & $1,1,2,3$ & -4 & 17 \\
\hline
\end{tabular}




\section{CHAPTER III}

\section{The Detailed Calculations}

The sections are numbered identically to the numbering of the weight-vectors in [Yonemura]. The title of each section is the weight-vector itself, and in each section is a representative polynomial from the family, taken from [Yonemura]. Table 3.1 gives the results of the calculations; $a^{\star}$ by a mirror-form indicates that there is a note about its existence or uniqueness at the end of the table.

Note that families with $\rho=15$ do not have mirrors on the list (because no family has $\rho=5)$.

Table 3.1: Results for the 95 Families and Their Mirrors

\begin{tabular}{|c|c|c|c|c|c|}
\hline No. & \multicolumn{2}{|l|}{ Rank } & \multicolumn{2}{|c|}{ Mirror Lattice and Family } & Weights \\
\hline 1 & $\rho=1$ & $\langle 4\rangle$ & $\left(E_{8}\right)^{2} \perp\langle-4\rangle \perp U$ & 56,73 & $(1,1,1,1)$ \\
\hline 2 & $\rho=12$ & $E_{6} \perp D_{4} \perp U(3)$ & $D_{4} \perp A_{2} \perp U(3)$ & not on list & $(2,3,3,4)$ \\
\hline 3 & $\rho=4$ & $M_{(1,1,1),(1,1,1), 0}$ & $\begin{array}{l}E_{8} \perp D_{4} \perp A_{2} \perp \\
U\end{array}$ & not on list & $(1,1,2,2)$ \\
\hline 4 & $\rho=10$ & $T_{4,4,4}$ & $T_{4,4,4}$ & 4 & $(1,3,4,4)$ \\
\hline 5 & $\rho=1$ & $\langle 2\rangle$ & $\left(E_{8}\right)^{2} \perp A_{1} \perp U$ & 52 , tetra. & $(1,1,1,3)$ \\
\hline 6 & $\rho=6$ & $D_{4} \perp U(2)$ & $D_{8} \perp D_{4} \perp U$ & $26,34,76$ & $(1,2,2,5)$ \\
\hline 7 & $\rho=3$ & $M_{(1,1),(1,1), 0}$ & $E_{8} \perp D_{7} \perp U$ & 64 & $(1,1,2,4)$ \\
\hline
\end{tabular}




\begin{tabular}{|c|c|c|c|c|c|}
\hline \multicolumn{6}{|c|}{ continued from previous page } \\
\hline \multirow{2}{*}{$\frac{\text { No. }}{8}$} & \multicolumn{2}{|r|}{$\operatorname{Pic}(S)$} & \multicolumn{2}{|c|}{ Mirror Lattice and Family } & \multirow{2}{*}{$\frac{\text { Weights }}{(1,2,3,6)}$} \\
\hline & $\rho=7$ & $M_{(1,1,2,2),(1,1,1,1),-2}$ & $q=w_{3,1}^{1} \perp w_{2,2}^{-1}$ & not on list & \\
\hline 9 & $\rho=10$ & $T_{2,5,5}$ & $T_{2,5,5}$ & 9,71 & $(1,4,5,10)$ \\
\hline 10 & $\rho=2$ & $U$ & $\left(E_{8}\right)^{2} \perp U$ & $65,46,80$ & $(1,1,4,6)$ \\
\hline 11 & $\rho=12$ & $E_{6} \perp D_{4} \perp U$ & $D_{4} \perp A_{2} \perp U$ & 24 & $(2,3,10,15)$ \\
\hline 12 & $\rho=6$ & $D_{4} \perp U$ & $E_{8} \perp D_{4} \perp U$ & 27,49 & $(1,2,9,6)$ \\
\hline 13 & $\rho=8$ & $E_{6} \perp U$ & $E_{8} \perp A_{2} \perp U$ & 20,59 & $(1,3,8,12)$ \\
\hline 14 & $\rho=10$ & $E_{8} \perp U$ & $E_{8} \perp U$ & $14,28,45,51$ & $(1,6,14,21)$ \\
\hline 15 & $\rho=14$ & $E_{6} \perp\left(A_{2}\right)^{3} \perp U$ & $\left(A_{2}\right)^{2} \perp U(3)$ & not on list & $(3,3,4,5)$ \\
\hline 16 & $\rho=16$ & $E_{8} \perp\left(A_{2}\right)^{3} \perp U$ & $A_{2} \perp U(3)$ & not on list & $(3,6,7,8)$ \\
\hline 17 & $\rho=14$ & $T_{2,5,5} \perp A_{4}$ & $A_{4} \perp\left(\begin{array}{cc}2 & 1 \\
1 & -2\end{array}\right.$ & not on list & $(2,3,5,5)$ \\
\hline 18 & $\rho=8$ & $M_{(1,2,2,2),(1,1,1,1),-2}$ & $q=w_{3,2}^{1} \perp w_{3,1}^{1}$ & not on list & $(1,2,3,3)$ \\
\hline 19 & $\rho=7$ & $M_{(1,1,1,1,2),(1,1,1,1,1),-2}$ & $q=v \perp w_{2,3}^{1}$ & not on list & $(1,2,2,3)$ \\
\hline 20 & $\rho=12$ & $E_{8} \perp A_{2} \perp U$ & $E_{6} \perp U$ & 13,72 & $(1,6,8,9)$ \\
\hline 21 & $\rho=2$ & $\left(\begin{array}{cc}2 & 1 \\
1 & -2\end{array}\right)$ & $E_{8} \perp T_{2,5,5}$ & 30,86 & $(1,1,1,2)$ \\
\hline 22 & $\rho=10$ & $E_{6} \perp A_{2} \perp U$ & $E_{6} \perp A_{2} \perp U$ & 22 & $(1,3,5,6)$ \\
\hline 23 & $\rho=11$ & $D_{5} \perp D_{4} \perp U(2)$ & $D_{4} \perp A_{3} \perp U(2)$ & not on list & $(2,2,3,5)$ \\
\hline 24 & $\rho=8$ & $D_{4} \perp A_{2} \perp U$ & $E_{6} \perp D_{4} \perp U$ & 11 & $(1,2,4,5)$ \\
\hline 25 & $\rho=4$ & $A_{2} \perp U$ & $E_{8} \perp E_{6} \perp U$ & $43,48,88$ & $(1,1,3,4)$ \\
\hline 26 & $\rho=14$ & $D_{8} \perp D_{4} \perp U$ & $D_{4} \perp U(2)$ & 6 & $(2,4,5,9)$ \\
\hline 27 & $\rho=14$ & $E_{8} \perp D_{4} \perp U$ & $D_{4} \perp U$ & 12 & $(2,3,8,11)$ \\
\hline 28 & $\rho=10$ & $E_{8} \perp U$ & $E_{8} \perp U$ & $14,28,45,51$ & $(1,3,7,10)$ \\
\hline 29 & $\rho=16$ & $T_{2,5,5} \perp D_{6}$ & $q=w_{5,1}^{-1} \perp\left(w_{2,1}^{-1}\right)^{2}$ & not on list & $(4,5,6,15)$ \\
\hline 30 & $\rho=18$ & $E_{8} \perp T_{2,5,5}$ & $\left(\begin{array}{cc}2 & 1 \\
1 & -2\end{array}\right) \star$ & 21 & $(5,7,8,20)$ \\
\hline 31 & $\rho=15$ & $E_{6} \perp A_{7} \perp U$ & $q=w_{2,3}^{-1} \perp w_{3,1}^{1} \star$ & not on list & $(3,4,5,12)$ \\
\hline 32 & $\rho=10$ & $D_{4} \perp D_{4} \perp U(2)$ & $D_{4} \perp D_{4} \perp U(2)$ & 32 & $(2,2,3,7)$ \\
\hline 33 & $\rho=12$ & $\begin{array}{l}M_{(1,1,1,1,2,2,3)} \\
(1,1,1,1,1,1,1),-4\end{array}$ & $\begin{array}{l}q=w_{3,1}^{1} \perp v \perp \\
w_{2,1}^{1} \perp w_{2,1}^{-1}\end{array}$ & not on list & $(2,3,4,9)$ \\
\hline 34 & $\rho=14$ & $D_{8} \perp D_{4} \perp U$ & $D_{4} \perp U(2)$ & 6 & $(2,6,7,15)$ \\
\hline 35 & $\rho=16$ & $E_{8} \perp A_{6} \perp U$ & $M_{(1,2),(1,1), 0}$ & 66 & $(3,4,7,14)$ \\
\hline 36 & $\rho=13$ & $T_{2,5,5} \perp A_{3}$ & $D_{5} \perp\left(\begin{array}{cc}2 & 1 \\
1 & -2\end{array}\right)$ & not on list & $(2,3,5,10)$ \\
\hline 37 & $\rho=9$ & $T_{3,4,4}$ & $T_{2,5,6}$ & 58 & $(1,3,4,8)$ \\
\hline 38 & $\rho=11$ & $E_{8} \perp A_{1} \perp U$ & $E_{7} \perp U$ & 50,82 & $(1,6,8,15)$ \\
\hline
\end{tabular}




\begin{tabular}{|c|c|c|c|c|c|}
\hline \multicolumn{6}{|c|}{ continued from previous page } \\
\hline \multirow{2}{*}{$\frac{\text { No. }}{39}$} & \multicolumn{2}{|l|}{ Rank } & \multicolumn{2}{|c|}{ Mirror Lattice and Family } & \multirow{2}{*}{$\frac{\text { Weights }}{(1,3,5,9)}$} \\
\hline & $\rho=9$ & $E_{6} \perp A_{1} \perp U$ & $E_{7} \perp A_{2} \perp U$ & 60 & \\
\hline 40 & $\rho=7$ & $D_{4} \perp A_{1} \perp U$ & $E_{7} \perp D_{4} \perp U$ & 81 & $(1,2,4,7)$ \\
\hline 41 & $\rho=13$ & $E_{6} \perp D_{5} \perp U$ & $A_{3} \perp A_{2} \perp U$ & not on list & $(2,3,7,12)$ \\
\hline 42 & $\rho=3$ & $A_{1} \perp U$ & $E_{8} \perp E_{7} \perp U$ & $68,83,92$ & $(1,1,3,5)$ \\
\hline 43 & $\rho=16$ & $E_{8} \perp E_{6} \perp U$ & $A_{2} \perp U$ & 25 & $(3,4,11,18)$ \\
\hline 44 & $\rho=7$ & $D_{5} \perp U$ & $E_{8} \perp A_{3} \perp U$ & not on list & $(1,2,5,8)$ \\
\hline 45 & $\rho=10$ & $E_{8} \perp U$ & $E_{8} \perp U$ & $14,28,45,51$ & $(1,4,9,14)$ \\
\hline 46 & $\rho=18$ & $E_{8}^{2} \perp U$ & $U$ & 10 & $(5,6,22,33)$ \\
\hline 47 & $\rho=15$ & $E_{7} \perp E_{6} \perp U$ & $A_{2} \perp A_{1} \perp U$ & not on list & $(3,4,14,21)$ \\
\hline 48 & $\rho=16$ & $E_{8} \perp E_{6} \perp U$ & $A_{2} \perp U$ & 25 & $(3,5,16,24)$ \\
\hline 49 & $\rho=14$ & $E_{8} \perp D_{4} \perp U$ & $D_{4} \perp U$ & 12 & $(2,5,14,21)$ \\
\hline 50 & $\rho=9$ & $E_{7} \perp U$ & $E_{8} \perp A_{1} \perp U$ & 38,77 & $(1,4,10,15)$ \\
\hline 51 & $\rho=10$ & $E_{8} \perp U$ & $E_{8} \perp U$ & $14,28,45,51$ & $(1,5,12,18)$ \\
\hline 52 & $\rho=19$ & $\begin{array}{l}E_{8} \perp D_{9} \perp U \cong \\
\left(E_{8}\right)^{2} \perp\langle-4\rangle \perp U\end{array}$ & $\langle 4\rangle, q=w_{2,2}^{1} \star$ & 5 & $(7,8,9,12)$ \\
\hline 53 & $\rho=15$ & $\begin{array}{l}M_{(1,2,2,2,3,4)} \\
(1,1,1,1,1,1),-4\end{array}$ & $\begin{array}{l}q=w_{3,2}^{1} \perp w_{3,1}^{1} \perp \\
w_{2,1}^{-1}\end{array}$ & not on list & $(3,4,5,6)$ \\
\hline 54 & $\rho=16$ & $E_{8} \perp\left(A_{2}\right)^{3} \perp U$ & $A_{2} \perp U(3)$ & not on list & $(3,5,6,7)$ \\
\hline 55 & $\rho=15$ & $D_{9} \perp D_{4} \perp U$ & $w_{2,2}^{1} \perp u$ & not on list & $(2,5,6,7)$ \\
\hline 56 & $\rho=19$ & $E_{8}^{2} \perp A_{1} \perp U$ & $\langle 2\rangle, q=w_{2,1}^{1} \star$ & 1 & $(5,6,8,11)$ \\
\hline 57 & $\rho=17$ & $\underset{U}{E_{8}} \perp D_{5} \perp A_{2} \perp$ & $w_{2,2}^{5} \perp w_{3,1}^{-1}$ & not on list & $(4,5,6,9)$ \\
\hline 58 & $\rho=11$ & $T_{2,5,6}$ & $T_{3,4,4}$ & 37 & $(1,4,5,6)$ \\
\hline 59 & $\rho=12$ & $E_{8} \perp A_{2} \perp U$ & $E_{6} \perp U$ & 13,72 & $(1,5,7,8)$ \\
\hline 60 & $\rho=11$ & $E_{7} \perp A_{2} \perp U$ & $E_{6} \perp A_{1} \perp U$ & 39 & $(1,4,6,7)$ \\
\hline 61 & $\rho=18$ & $E_{8} \perp D_{8} \perp U$ & $U(2)$ & not on list & $(4,6,7,11)$ \\
\hline 62 & $\rho=16$ & $D_{9} \perp D_{5} \perp U$ & $q=w_{2,2}^{1} \perp w_{2,2}^{5}$ & not on list & $(3,4,5,8)$ \\
\hline 63 & $\rho=8$ & $M_{(1,1,2,3),(1,1,1,1),-2}$ & $T_{2,5,5} \perp\left(A_{1}\right)^{2}$ & not on list & $(1,2,3,4)$ \\
\hline 64 & $\rho=17$ & $E_{8} \perp D_{7} \perp U$ & $M_{(1,1),(1,1), 0}$ & 7 & $(3,4,7,10)$ \\
\hline 65 & $\rho=18$ & $E_{8}^{2} \perp U$ & $U$ & 10 & $(3,5,11,14)$ \\
\hline 66 & $\rho=4$ & $M_{(1,2),(1,1), 0}$ & $E_{8} \perp A_{6} \perp U$ & 35 & $(1,1,2,3)$ \\
\hline 67 & $\rho=14$ & $\begin{array}{l}E_{6}^{2} \perp U \cong E_{8} \perp \\
\left(A_{2}\right)^{2} \perp U\end{array}$ & $\left(A_{2}\right)^{2} \perp U$ & not on list & $(2,3,7,9)$ \\
\hline 68 & $\rho=17$ & $E_{8} \perp E_{7} \perp U$ & $A_{1} \perp U$ & 42 & $(3,4,10,13)$ \\
\hline 69 & $\rho=13$ & $D_{4} \perp A_{7} \perp U$ & $q=w_{2,3}^{-1} \perp v^{\star}$ & not on list & $(2,3,4,7)$ \\
\hline 70 & $\rho=14$ & $\begin{array}{l}E_{8} \perp A_{2} \perp \\
\left(A_{1}\right)^{2} \perp U\end{array}$ & $q=w_{3,1}^{-1} \perp\left(w_{2,1}^{1}\right)^{2}$ & not on list & $(2,3,5,8)$ \\
\hline & & & & continues & on next page \\
\hline
\end{tabular}




\begin{tabular}{|c|c|c|c|c|c|}
\hline \multicolumn{6}{|c|}{ continued from previous page } \\
\hline \multirow{2}{*}{$\frac{\text { No. }}{71}$} & \multicolumn{2}{|l|}{ Rank } & \multicolumn{2}{|c|}{ Mirror Lattice and Family } & \multirow{2}{*}{$\frac{\text { Weights }}{(1,3,4,7)}$} \\
\hline & $\rho=10$ & $T_{2,5,5}$ & $T_{2,5,5}$ & 9,71 & \\
\hline 72 & $\rho=8$ & $E_{6} \perp U$ & $E_{8} \perp A_{2} \perp U$ & 20,59 & $(1,2,5,7)$ \\
\hline 73 & $\rho=19$ & $E_{8}^{2} \perp A_{1} \perp U$ & $\langle 2\rangle, q=w_{2,1}^{1} \star$ & 1 & $(7,8,10,25)$ \\
\hline 74 & $\rho=17$ & $M_{(3,3,4,6),(1,1,1,3),-4}$ & $q=w_{2,3}^{-5 \star}$ & not on list & $(4,5,7,16)$ \\
\hline 75 & $\rho=13$ & $E_{7} \perp\left(A_{1}\right)^{4} \perp U$ & $\left(A_{1}\right)^{5} \perp U$ & not on list & $(2,4,5,11)$ \\
\hline 76 & $\rho=14$ & $D_{8} \perp D_{4} \perp U$ & $D_{4} \perp U(2)$ & 6 & $(2,5,6,13)$ \\
\hline 77 & $\rho=11$ & $E_{8} \perp A_{1} \perp U$ & $E_{7} \perp U$ & 50,82 & $(1,5,7,13)$ \\
\hline 78 & $\rho=10$ & $E_{7} \perp A_{1} \perp U$ & $E_{7} \perp A_{1} \perp U$ & 78 & $(1,4,6,11)$ \\
\hline 79 & $\rho=15$ & $E_{8} \perp D_{5} \perp U$ & $A_{3} \perp U$ & not on list & $(2,5,9,16)$ \\
\hline 80 & $\rho=18$ & $E_{8}^{2} \perp U$ & $U$ & 10 & $(4,5,13,22)$ \\
\hline 81 & $\rho=13$ & $E_{8} \perp\left(A_{1}\right)^{3} \perp U$ & $D_{4} \perp A_{1} \perp U$ & 40 & $(2,3,8,13)$ \\
\hline 82 & $\rho=9$ & $E_{7} \perp U$ & $E_{8} \perp A_{1} \perp U$ & 38,77 & $(1,3,7,11)$ \\
\hline 83 & $\rho=17$ & $E_{8} \perp E_{7} \perp U$ & $A_{1} \perp U$ & 42 & $(4,5,18,27)$ \\
\hline 84 & $\rho=18$ & $E_{8} \perp A_{8} \perp U$ & $q=w_{3,2}^{-1 \star}$ & not on list & $(5,6,7,9)$ \\
\hline 85 & $\rho=13$ & $\underset{U}{D_{4} \perp A_{6} \perp A_{1} \perp}$ & $D_{4} \perp\langle 14\rangle \perp U$ & not on list & $(2,3,4,5)$ \\
\hline 86 & $\rho=18$ & $E_{8} \perp T_{2,5,5}$ & $\left(\begin{array}{cc}2 & 1 \\
1 & -2\end{array}\right)$ & 21 & $(4,5,7,9)$ \\
\hline 87 & $\rho=10$ & $T_{3,4,5}$ & $T_{3,4,5}$ & 87 & $(1,3,4,5)$ \\
\hline 88 & $\rho=16$ & $E_{8} \perp E_{6} \perp U$ & $A_{2} \perp U$ & not on list & $(2,5,9,11)$ \\
\hline 89 & $\rho=8$ & $M_{(1,2,4),(1,1,2),-2}$ & $A_{10} \perp U$ & not on list & $(1,2,3,5)$ \\
\hline 90 & $\rho=17$ & 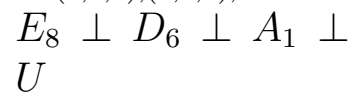 & $A_{1} \perp U(2)$ & not on list & $(4,6,7,17)$ \\
\hline 91 & $\rho=18$ & 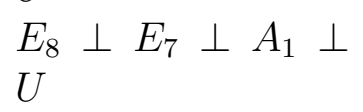 & $q=w_{2,1}^{1} \perp w_{2,1}^{-1}$ & not on list & $(5,6,8,19)$ \\
\hline 92 & $\rho=17$ & $E_{8} \perp E_{7} \perp U$ & $A_{1} \perp U$ & 42 & $(3,5,11,19)$ \\
\hline 93 & $\rho=16$ & $E_{8} \perp D_{6} \perp U$ & $\left(A_{1}\right)^{2} \perp U$ & not on list & $(3,4,10,17)$ \\
\hline 94 & $\rho=16$ & $M_{(2,3,4,6),(1,1,2,2),-4}$ & $q=w_{19,1}^{1}$ & not on list & $(3,4,5,7)$ \\
\hline 95 & $\rho=14$ & $M_{(1,2,4,6),(1,1,2,3),-4}$ & $q=w_{17,1}^{-1}$ & not on list & $(2,3,5,7)$ \\
\hline
\end{tabular}

\subsubsection{Existence and Uniqueness of Mirror Lattices}

There is a paper [Mir-Mor] by Miranda and Morrison, which helps us determine when a lattice is unique, in cases where $\rho \geq 3$ but Nikulin's criteria do not apply. The authors compute the number of equivalence classes of primitive embeddings into a unimodular lattice. Of course, if this number is 1 , the lattice is unique. [Venkov] states that any rank 2 form of determinant 4 is unique up to isomorphism. These 
are enough to show that almost all of the mirror lattices are unique. Here are the exceptions:

30 - It exists because we have found the mirror and is unique [MirE].

31 - We are unable to check the existence of the mirror lattice because we know of no existing lattice of any rank with this form.

52 - It exists because we found the mirror. It is not unique.

56 - It exists because we found the mirror. It is not unique.

69 - We are unable to check the existence of the mirror lattice because we know of no existing lattice of any rank with this form.

73 - It exists because we found the mirror. It is not unique.

74 - We are unable to check the existence of the mirror lattice because we know of no existing lattice of any rank with this form.

84 - We are unable to check the existence of the mirror lattice because we know of no existing lattice of any rank with this form. If it exists, it is not unique.

For each of the fibrations discussed below, I have checked to see every curve of genus $\geq 2$ has equal intersection multiplicity with each fibre.

3.0.1.1 Definition. A fibration has good rank if it satisfies the Shioda-Tate formula with $r k(M W)=0$.

\section{$3.1(1,1,1,1)$}

A representative equation for this family of hypersurfaces is $x^{4}+y^{4}+z^{4}+w^{4}$. This was already calculated by Dolgachev to have $\operatorname{Pic}(S)=\langle 4\rangle$, and the polytope is nonsingular anyway; thus, the computer programs would produce nothing. 


\section{$3.2(2,3,3,4)$}

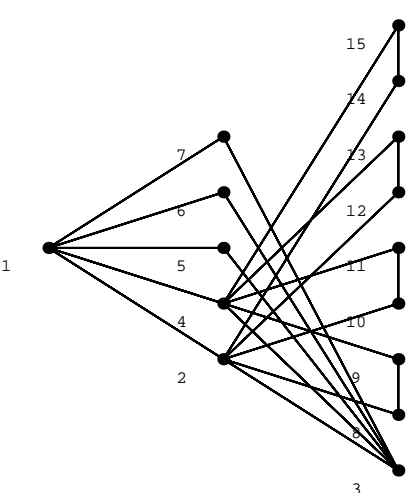

A representative equation for this family of hypersurfaces is $x^{6}+y^{4}+z^{4}+w^{3}$.

The Fibration. $\rho=12$; curves 1,3 , and 4 have genus 1 and the edges between 1 and 4 and between 3 and 4 have multiplicity 2 . No fibration of good rank admits a section.

A Non-Jacobian Fibration. If we compute the discriminant of the corresponding matrix, we have $108=27 \cdot 4$, so we expect to either find a fibration with discriminant 27 or discriminant 12 . We begin with the natural $\tilde{E}_{6}$ formed by curves $2,8-13$. This means that curves $1,3,4$, and 14 are 3 -sections. So, to get a fibration with disc. $=$ 27 we need to form a lattice of discriminant 9 and rank 4 (impossible) and to get a fibration with disc. $=12$ we need to form something of discriminant 4 and rank 4 - this could only be $\tilde{D}_{4}$, we may form one by adding a curve which intersects the remaining four curves. The corresponding Jacobian fibration has good rank.

Method: Mukai and Matrix. We can apply Mukai's theorem to see that $\operatorname{Pic}(S)$ is of index 3 in $\operatorname{Pic}(\mathbf{J}(S))$, so that $\operatorname{disc} \operatorname{Pic}(S)=9 \cdot \operatorname{Pic}(\mathbf{J}(S))$ and the intersection matrix for our graph determines $\operatorname{Pic}(S)$.

Calculate the Form. We have two value-1 generators of order 2 ; their sum is 1 , so they must correspond to the form $v$. We have three value- $2 / 3$ generators of order 3 , and at least one of these must correspond to a $w_{3,1}^{-1}$. We have 5 value- 1 order 6 
generators; each must have a component of order 3 and of order 2 , and $v$ has generator values of 1 only so the order 3 component must have value 0 , which could be $2 / 3+2 / 3+2 / 3$ or $4 / 3+2 / 3$. There must be a $4 / 3$-valued generator in order to embed this lattice into $w_{3,1}^{-1} \perp v$, so that we have the form $\left(w_{3,1}^{-1}\right)^{2} \perp w_{3,1}^{1} \perp v$. This corresponds to $E_{6} \perp D_{4} \perp U(3)$.

\section{$3.3(1,1,2,2)$}

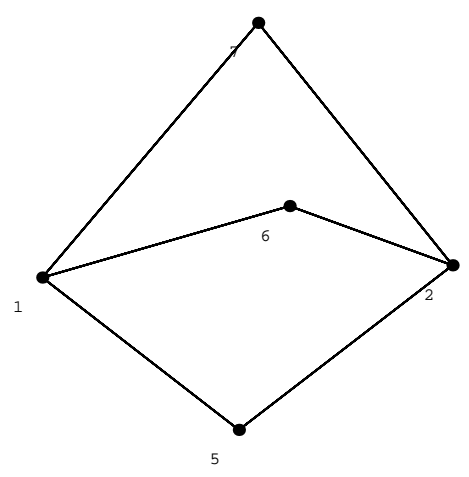

A representative equation for this family of hypersurfaces is $x^{6}+y^{6}+z^{3}+w^{3}$.

The Fibration. $\rho=4$; curves 1 and 2 have genus 1 . There is no fibration which has good rank. Therefore $M W$ is infinite and we will have to consult the intersection matrix of the graph.

Method: Matrix Calculations. The matrix has discriminant 12, and there are 4 generators. There are two 1-value generators of order 2 ; their sum has value 1 , so this indicates we have form $v$. Then, we have a a $5 / 3$-value generator of order 6 ; the value $5 / 3$ must be $1+2 / 3$ and so our form is $v \perp w_{3,1}^{-1}$. This has no isotropic subgroups, so it must have index 1 in $\operatorname{Pic}(S)$. This lattice has no representation as a sum of Dynkin lattices. However, we can express this form as $M_{(1,1,1),(1,1,1), 0}$. 


\section{$3.4(1,3,4,4)$}

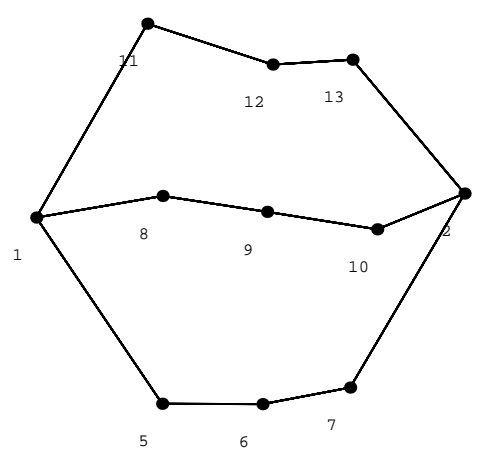

A representative equation for this family of hypersurfaces is $x^{12}+y^{4}+z^{3}+w^{3}$. This is one of Arnold's 14 original surface singularities. Curve 2 is elliptic, and the remaining curves form the lattice $\operatorname{Pic}(S)=T_{4,4,4}$. This lattice has no representation as a sum of Dynkin lattices.

\section{$3.5(1,1,1,3)$}

A representative equation for this family of hypersurfaces is $x^{6}+y^{6}+z^{6}+w^{2}$. The polytope is nonsingular, and $\operatorname{Pic}(S)$ was already calculated by Dolgachev.

\section{$3.6(1,2,2,5)$}

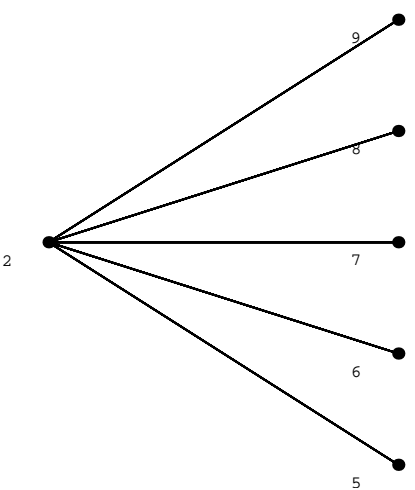


A representative equation for this family of hypersurfaces is $x^{10}+y^{5}+z^{5}+w^{2}$.

The Fibration. $\rho=6$; curves 2, 6-9 form a $\tilde{D}_{4}$ and then curve 5 is a 2 -section. This is the only fibration we have, and as these fibres are the same for the Jacobian fibration, we see that $\mathbf{J}(S)$ has good rank.

Method: Mukai. We apply the results of Mukai to see that $\operatorname{Pic}(S)$ has index 2 in $\operatorname{Pic}(\mathbf{J}(S)$, so we need to consult the matrix.

Matrix Calculations. The matrix has discriminant 16. We have one 0-value generator of order 2 and 4 1-value generators of order 2 . Therefore the discriminant group must be $\left(\mathbb{Z}_{2}\right)^{4}$. See Appendix 1 for the possible forms. Because $\operatorname{Pic}(\mathbf{J}(S)$ has discriminant form $v, \operatorname{Pic}(S)$ must embed in $v$. By examining the isotropic subgroups of each possibility, we see that the only two which embed into $v$ are $u \perp v$ and $v \perp w_{2,1}^{1} \perp w_{2,1}^{-1}$. Now we need to determine which of these corresponds to the generators we have. Mathematica tells us that the sum of the 0-value generator with one of the 1 -value generators has value 0 . Such elements exist in $u \perp v$ but not in $v \perp w_{2,1}^{1} \perp w_{2,1}^{-1}$. Therefore $\operatorname{Pic}(S)=u \perp v$. This lattice has no representation as a sum of Dynkin lattices.

\section{$3.7 \quad(1,1,2,4)$}

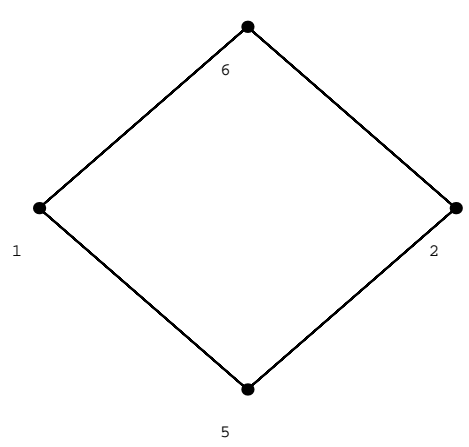


A representative equation for this family of hypersurfaces is $x^{8}+y^{8}+z^{4}+w^{2}$.

The Fibration. $\rho=3$; there is no fibration which has good rank. Therefore $M W$ must be infinite.

Method: Matrix Calculations. The matrix has discriminant 4 and has a 7/4value generator of order 4 ; thus, the form must be $w_{2,2}^{-1}$. This form has no isotropic subgroups, so $\operatorname{Pic}(S)=w_{2,2}^{-1}$. This lattice has no representation as a sum of Dynkin lattices. However, we can express this form as $M_{(1,1),(1,1), 0}$.

\section{$3.8(1,2,3,6)$}

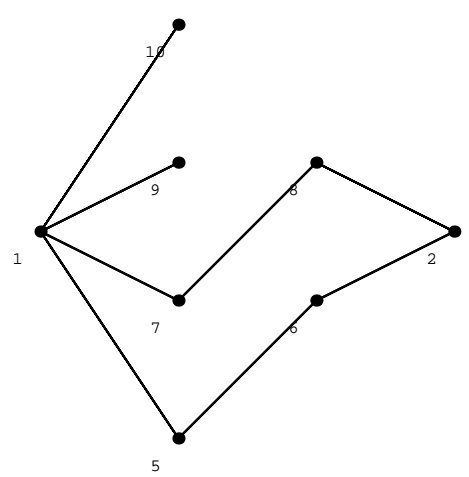

A representative equation for this family of hypersurfaces is $x^{12}+y^{6}+z^{4}+w^{2}$.

The Fibration. $\rho=7$; there is no fibration which has good rank, so $M W$ is infinite. Method: Matrix Calculations. This has discriminant 12 . There is a $1 / 4-$ value generator of order 4 and a $2 / 3$-value generator of order 3 . They are independent, so our form is $w_{2,2}^{1} \perp w_{3,1}^{-1}$. This form has no isotropic subgroups, so $\operatorname{Pic}(S)=w_{2,2}^{1} \perp w_{3,1}^{-1}$. This lattice has no representation as a sum of Dynkin lattices. However, we can express this form as $M_{(1,1,2,2),(1,1,1,1),-2}$. 


\section{$3.9(1,4,5,10)$}

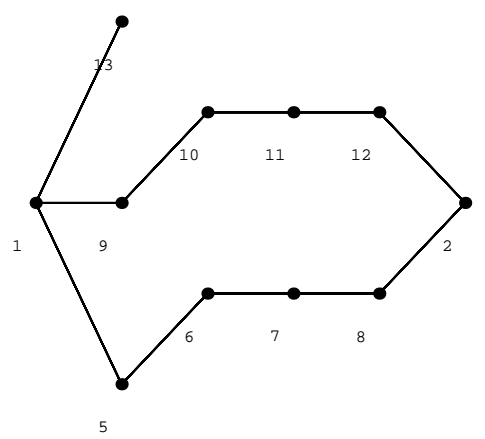

A representative equation for this family of hypersurfaces is $x^{20}+y^{5}+z^{4}+w^{2}$.

This is one of Arnold's 14 original surface singularities. $\rho=10$. Curve 2 has genus

1. Note that aside from curve 2, we have $\operatorname{Pic}(S)=T_{2,5,5}$. (It corresponds to form $q_{5,1}^{-1}$.) This lattice has no representation as a sum of Dynkin lattices.

\section{$3.10(1,1,4,6)$}

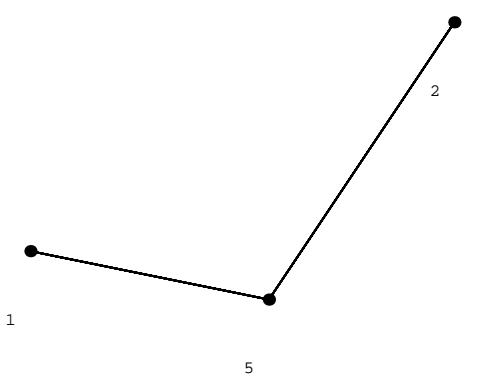

A representative equation for this family of hypersurfaces is $x^{12}+y^{12}+z^{3}+w^{2}$.

$\rho=2$. Curves 1 and 2 have genus 1 ; if we consider these to be fibres, then curve 5 is a section. Therefore $\operatorname{Pic}(S)=U$. 


\section{$3.11 \quad(2,3,10,15)$}

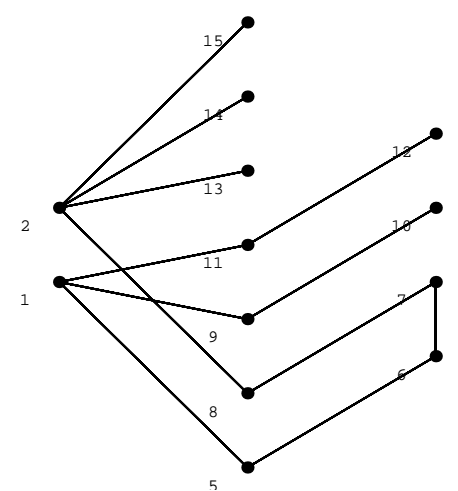

A representative equation for this family of hypersurfaces is $x^{15}+y^{10}+z^{3}+w^{2}$. $\rho=12$. None of the curves has genus 1 , so we may form curves $2,8,13,14,15$ into a $\tilde{D}_{4}$, so that curve 7 is a section and curves $1,5,6,9-12$ form an $\tilde{E}_{6}$. This fibration has good rank, and we have exhibited one section; furthermore, the form associated with this fibration has no isotropic subgroups. Therefore, $\operatorname{Pic}(S)=D_{4} \perp E_{6} \perp U$.

\section{$3.12(1,2,6,9)$}

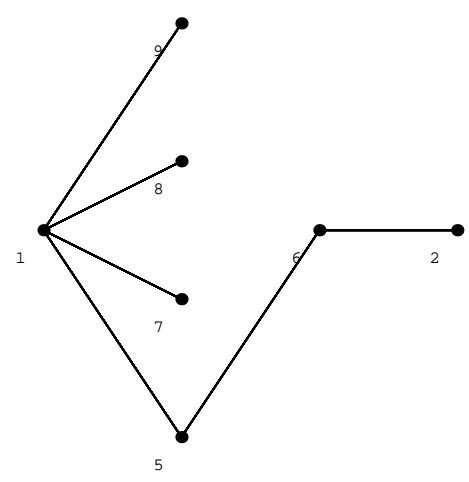

A representative equation for this family of hypersurfaces is $x^{18}+y^{9}+z^{3}+w^{2}$. $\rho=6$ and curve 2 has genus 1 . If we consider curve 2 as a fibre, then curve 6 is a section and curves $1,5,7-9$ form a $\tilde{D}_{4}$. This fibration has good rank, and the associated form $v$ has no isotropic subgroups, so $\operatorname{Pic}(S)=D_{4} \perp U$. 


\section{$3.13(1,3,8,12)$}

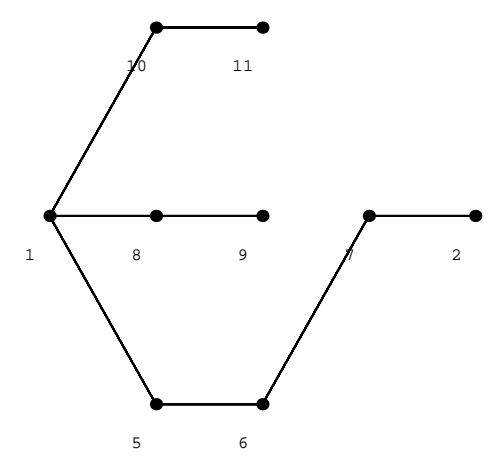

A representative equation for this family of hypersurfaces is $x^{24}+y^{8}+z^{3}+w^{2}$.

This is one of Arnold's 14 original surface singularities. $\rho=8$ and curve 2 has genus 1. If we consider curve 2 to be a fibre, then curve 7 is a section and we are left with a $\tilde{E}_{6}$. This fibration has good rank and the associated form has no isotropic subgroups so $\operatorname{Pic}(S)=E_{6} \perp U$.

\section{$3.14(1,6,14,21)$}

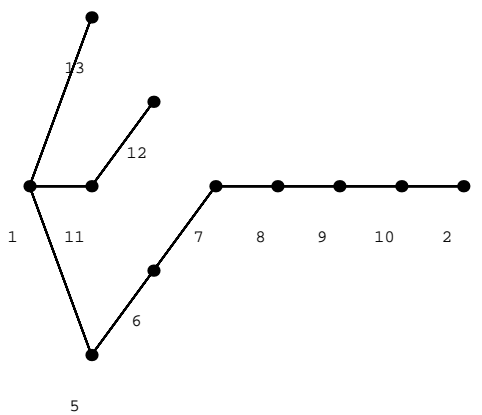

A representative equation for this family of hypersurfaces is $x^{42}+y^{7}+z^{3}+w^{2}$.

This is one of Arnold's 14 original surface singularities. $\rho=10$ and curve 2 has genus 1. If we consider this to be a fibre, then curve 10 is a section and we are left with $\tilde{E}_{8}$. This has good rank and is unimodular so $\operatorname{Pic}(S)=E_{8} \perp U$. 


\section{$3.15(3,3,4,5)$}

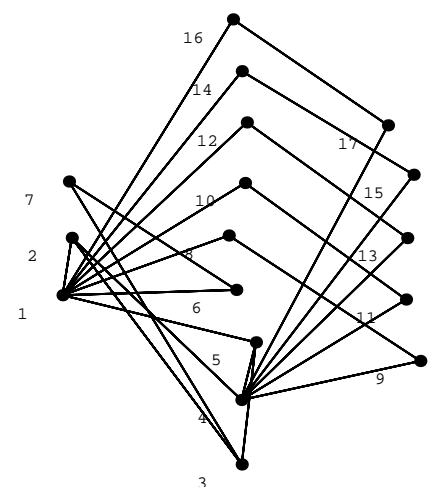

A representative equation for this family of hypersurfaces is $-x^{5}+y^{5}+x z^{3}+y z^{3}+w^{3}$.

The Fibration. $\rho=14$ and curves 2 and 5 have genus 1 . If we consider these to be fibres, then curves 1,3 ,and 4 are sections and the most obvious way to complete the remaining fibres is to make $6 \tilde{A}_{2}$. This fibration has good rank, but we have exhibited three sections.

Method: Intermediate Lattice Calculation. The discriminant group of our lattice is $\left(\mathbb{Z}_{3}\right)^{6}$; there are scads of conjugate isotropic subgroups. It suffices to check representatives, which we choose to be $(1,1,1,0,0,0)$ and $(1,1,1,1,1,1)$. We will use Miranda's notation for this calculation.

Begin with $(1,1,1,0,0,0)$. We will suppress the last three entries and append $\left(w_{3,1}^{1}\right)^{3}$ to the result. There are ten elements now (up to permutation), and we determine which of these are perpendicular to $(1,1,1)$ by testing $q(a)-q(a+(1,1,1))=0$.

$$
\begin{gathered}
(0,0,0)-0-0=0 ;(0,0,1)-1 / 3-0=1 / 3 \\
(0,0,2)-1 / 3-2 / 3=-1 / 3 ;(0,1,1)-2 / 3-0=2 / 3 \\
(0,1,2)-2 / 3-2 / 3=0 ;(0,2,2)-2 / 3-1 / 3=1 / 3 \\
(1,1,1)-0-0=0 ;(1,1,2)-0-2 / 3=-2 / 3 \\
(1,2,2)-0-1 / 3=-1 / 3 ;(2,2,2)-0-0=0
\end{gathered}
$$


This leaves us with

$$
\begin{array}{llll}
(0,0,0) & (1,1,1) & (2,2,2) & 0 \\
(0,1,2) & (1,2,0) & (2,0,1) & -2 / 3 \\
(0,2,1) & (1,0,2) & (2,1,0) & -2 / 3
\end{array}
$$

where we have written out all the permutations of the elements, by coset, and their values. This corresponds to the form $w_{3,1}^{-1}$, so the result is $\left(w_{3,1}^{1}\right)^{3} \perp w_{3,1}^{-1}$.

Now we will work with $(1,1,1,1,1,1)$. Up to permutation, we have 28 distinct elements. We determine which are perpendicular to $(1,1,1,1,1,1)$; the columns are for the element, its value, the value when 111111 is added, and whether it is perpendicular or not.

$$
\begin{array}{ccccccccccccccc}
000000 & 0 & 0 & \mathrm{Y} & 000011 & 2 / 3 & 0 & \mathrm{~N} & 000111 & 0 & 0 & \mathrm{Y} \\
000001 & 1 / 3 & 0 & \mathrm{~N} & 000012 & 2 / 3 & -2 / 3 & \mathrm{Y} & 000112 & 0 & -2 / 3 & \mathrm{~N} \\
000002 & 1 / 3 & -2 / 3 & \mathrm{~N} & 000022 & 2 / 3 & -1 / 3 & \mathrm{~N} & & & & & & & \\
0
\end{array}
$$

This leaves us with (arranged by coset class, and still up to permutation): 


$\begin{array}{lllll}\text { elt } & \text { elt }+111111 & \text { elt }+222222 & \text { value } & \text { no. of such elts } \\ 000000 & 111111 & 222222 & 0 & 1 \\ 000012 & 011112 & 012222 & -2 / 3 & 30 \\ 000111 & 111222 & 000222 & 0 & 20 \\ 001122 & \text { (same) } & \text { (same) } & -1 / 3 & 30\end{array}$

So we wish to find which form on $\left(\mathbb{Z}_{3}\right)^{4}$ has 210 -values, $30-2 / 3$-values, and 30 -1/3-values. See Appendix 1 for the possibilities; the form must be $\left(w_{3,1}^{1}\right)^{3} \perp w_{3,1}^{-1}$. This is exactly what we found for the first isotropic subgroup, so $\operatorname{Pic}(S)=E_{6} \perp$ $\left(A_{2}\right)^{3} \perp U$.

\section{$3.16(3,6,7,8)$}

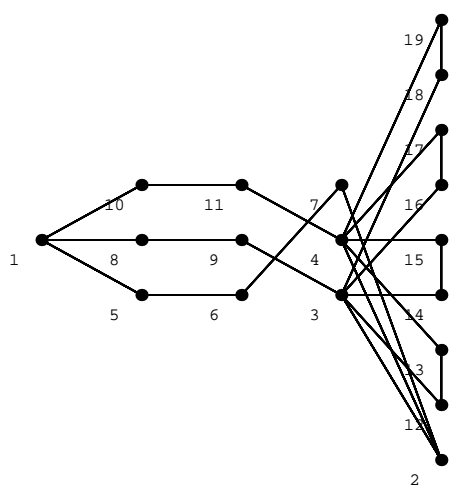

A representative equation for this family of hypersurfaces is $x^{8}+y^{4}+x z^{3}+w^{3}$.

The Fibration. $\rho=16$ and curve 2 has genus 1 . If we consider it to be a fibre, then curves 3,4 , and 7 are sections and we are left with $\tilde{E}_{6}+4 \tilde{A}_{2}$. This has good rank and we have exhibited three sections.

Method: Intermediate Lattice Calculation. Each fibre has discriminant group $\mathbb{Z}_{3}$, so we are beginning with $\left(\mathbb{Z}_{3}\right)^{5}$. We will use Miranda's notation; we have three distinct isotropic subgroups, $(1,1,0,0,0),(0,0,1,1,1)$, and $(1,1,1,1,1)$.

We begin with $(1,1,0,0,0)$, and will suppress the last three entries. There are nine 
elements now, and we'll determine which of these are perpendicular to $(1,1)$ by testing

$$
\begin{aligned}
& q(a)-q(a+(1,1))=0 .(0,0)-0-0=0 \\
& (0,1)-1 / 3-0=1 / 3 ;(0,2)-1 / 3-0=1 / 3 \\
& (1,0)-2 / 3-0=2 / 3 ;(1,1)-0-0=0 \\
& (1,2)-0-2 / 3=-2 / 3 ;(2,0)-2 / 3-1 / 3=1 / 3 \\
& (2,1)-0-1 / 3=-1 / 3 ;(2,2)-0-0=0
\end{aligned}
$$

This leaves us with $(0,0),(1,1)$, and $(2,2)$ which are all identified if we mod out by the subgroup generated by $(1,1)$. So we are left with $\left(w_{3,1}^{1}\right)^{3}$.

For $(0,0,1,1,1)$, we suppress the first two entries. There are 27 entries this time.

$$
\begin{array}{lll}
(0,0,0)-0-0=0 & (1,0,0)-1 / 3-0=1 / 3 & (2,0,0)-1 / 3-2 / 3=-1 / 3 \\
(0,0,1)-1 / 3-0=1 / 3 & (1,0,1)-2 / 3-0=2 / 3 & (2,0,1)-2 / 3-2 / 3=0 \\
(0,0,2)-1 / 3-2 / 3=-1 / 3 & (1,0,2)-2 / 3-2 / 3=0 & (2,0,2)-2 / 3-1 / 3=1 / 3 \\
(0,1,0)-1 / 3-0=1 / 3 & (1,1,0)-2 / 3-0=2 / 3 & (2,1,0)-2 / 3-2 / 3=0 \\
(0,1,1)-2 / 3-0=2 / 3 & (1,1,1)-0-0=0 & (2,1,1)-0-2 / 3=-2 / 3 \\
(0,1,2)-2 / 3-2 / 3=0 & (1,1,2)-0-2 / 3=-2 / 3 & (2,1,2)-0-1 / 3=-1 / 3 \\
(0,2,0)-1 / 3-2 / 3=-1 / 3 & (1,2,0)-2 / 3-2 / 3=0 & (2,2,0)-2 / 3-1 / 3=1 / 3 \\
(0,2,1)-2 / 3-2 / 3=0 & (1,2,1)-0-2 / 3=-2 / 3 & (2,2,1)-0-1 / 3=-1 / 3 \\
(0,2,2)-2 / 3-1 / 3=1 / 3 & (1,2,2)-0-1 / 3=-1 / 3 & (2,2,2)-0-0=0
\end{array}
$$

We arrange these by cosets of $(1,1,1)$ :

$\begin{array}{llll}(0,0,0) & (1,1,1) & (2,2,2) & \text { value is } 0 \\ (0,1,2) & (1,2,0) & (2,0,1) & \text { value is } 2 / 3 \\ (0,2,1) & (1,0,2) & (2,1,0) & \text { value is } 2 / 3\end{array}$

This is $\mathbb{Z}_{3}$ and the value indicates that we have $w_{3,1}^{-1}$ for a total of $w_{3,1}^{-1} \perp w_{3,1}^{-1} \perp w_{3,1}^{1}$ which, by the first isomorphism relation, is equivalent to $\left(w_{3,1}^{1}\right)^{3}$.

For $(1,1,1,1,1)$ : up to permutation, we have the following elements and values: 


$$
\begin{array}{lll}
(0,0,0,0,0)-0 & (1,0,0,0,0)-2 / 3 & (2,0,0,0,0)-2 / 3 \\
(0,0,0,0,1)-1 / 3 & (1,0,0,0,1)-0 & (2,0,0,0,1)-0 \\
(0,0,0,0,2)-1 / 3 & (1,0,0,0,2)-0 & (2,0,0,0,2)-0 \\
(0,0,0,1,1)-2 / 3 & (1,0,0,1,1)-1 / 3 & (2,0,0,1,1)-1 / 3 \\
(0,0,0,1,2)-2 / 3 & (1,0,0,1,2)-1 / 3 & (2,0,0,1,2)-1 / 3 \\
(0,0,0,2,2)-2 / 3 & (1,0,0,2,2)-1 / 3 & (2,0,0,2,2)-1 / 3 \\
(0,0,1,1,1)-0 & (1,0,1,1,1)-2 / 3 & (2,0,1,1,1)-2 / 3 \\
(0,0,1,1,2)-0 & (1,0,1,1,2)-2 / 3 & (2,0,1,1,2)-2 / 3 \\
(0,0,1,2,2)-0 & (1,0,1,2,2)-2 / 3 & (2,0,1,2,2)-2 / 3 \\
(0,0,2,2,2)-0 & (1,0,2,2,2)-2 / 3 & (2,0,2,2,2)-2 / 3 \\
(0,1,1,1,1)-1 / 3 & (1,1,1,1,1)-0 & (2,1,1,1,1)-0 \\
(0,1,1,1,2)-1 / 3 & (1,1,1,1,2)-0 & (2,1,1,1,2)-0 \\
(0,1,1,2,2)-1 / 3 & (1,1,1,2,2)-0 & (2,1,1,2,2)-0 \\
(0,1,2,2,2)-1 / 3 & (1,1,2,2,2)-0 & (2,1,2,2,2)-0 \\
(0,2,2,2,2)-1 / 3 & (1,2,2,2,2)-0 & (2,2,2,2,2)-0
\end{array}
$$

Now, because the condition for perpendicularity to $H$ is $q(a)-q(a+(1,1,1,1,1))=0$, which can be rewritten as $q(a)=q(a+(1,1,1,1,1))$, we just have to add $(1,1,1,1,1)$ to each element and see if the quadratic form value matches. Here's a list for which the condition holds (arranged, as usual, by coset):

$$
\begin{array}{lllll}
(0,0,0,0,0) & (1,1,1,1,1) & (2,2,2,2,2) & 1 \text { element } & \text { value 0 } \\
(0,0,0,1,2) & (1,0,1,1,2) & (2,0,1,2,2) & 12 \text { elements } & \text { value } 2 / 3 \\
(0,0,1,1,1) & (1,1,2,2,2) & (2,0,0,0,2) & 4 \text { elements } & \text { value } 0 \\
(0,0,2,2,2) & (1,0,0,0,1) & (2,1,1,1,2) & 4 \text { elements } & \text { value 0 } \\
(0,1,1,2,2) & (1,0,0,2,2) & (2,0,0,1,1) & 6 \text { elements } & \text { value } 1 / 3
\end{array}
$$


Each element (except the identity) is of order 3, so with 27 elements we're looking at $\mathbb{Z}_{3}^{3}$. By examining the possibilities in Appendix 1, we see that we have $\left(w_{3,1}^{1}\right)^{3}$. The Answer. This corresponds to $\operatorname{Pic}(S)=E_{8} \perp\left(A_{2}\right)^{3} \perp U$.

\section{$3.17 \quad(2,3,5,5)$}

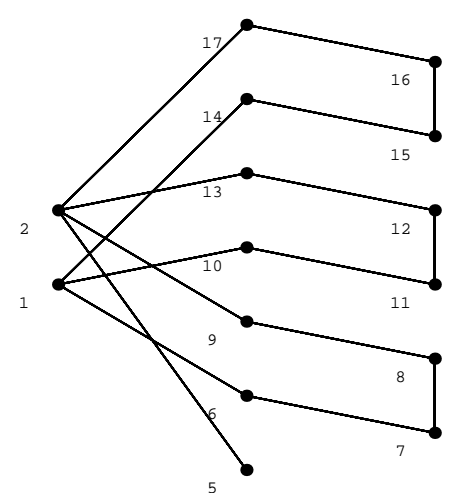

A representative equation for this family of hypersurfaces is $x^{6} y+x^{5} z+x^{5} w+y^{5}+$ $z^{3}+w^{3}$

$\rho=14$ and there are no curves of genus 1 . There are no fibrations with good rank.

Method: Matrix Calculation. The matrix has discriminant 25, and all generators have order 5 , so the discriminant group is $\left(\mathbb{Z}_{5}\right)^{2}$. There are a $6 / 5$-valued generator and a 8/5-valued generator which are independent of each other, so the form must be $w_{5,1}^{-1} \perp w_{5,1}^{1}$. This form has no isotropic subgroups, and the associated lattice of $\operatorname{rank} 14$ is $\operatorname{Pic}(S)=T_{2,5,5} \perp A_{4}$. 


\section{$3.18(1,2,3,3)$}

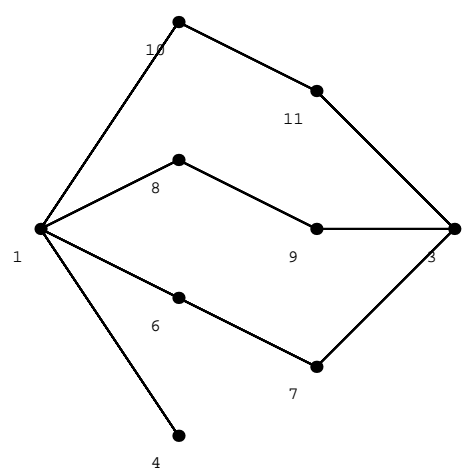

A representative equation for this family of hypersurfaces is $x^{9}+x y^{4}+y^{3} z+y^{3} w+$ $z^{3}+w^{3}$

The Fibration. $\rho=8$ and curve 3 has genus 1 . There is no Jacobian fibration of good rank, so we look at curves $1,6-11$ as forming a $\tilde{E}_{6}$, so that curves 3 and 4 are 3-sections. Thus, the associated Jacobian fibration has good rank.

Method: Mukai and Matrix. We can apply Mukai's theorem to see that $\operatorname{Pic}(S)$ is of index 3 in $\operatorname{Pic}(\mathbf{J}(S))$, so that disc $\operatorname{Pic}(S)=9 \cdot \operatorname{Pic}(\mathbf{J}(S))$ and the intersection matrix for our graph determines $\operatorname{Pic}(S)$. The matrix has discriminant 27 . We have order 9 generators with values of $14 / 9$ and $2 / 9$, so we have $w_{3,2}^{-1}$. We also have order 3 generators with value $2 / 3$ each, so we have $w_{3,1}^{-1}$.

The Answer. $\operatorname{Pic}(S)=w_{3,2}^{-1} \perp w_{3,1}^{-1}$. This lattice has no representation as a sum of Dynkin lattices. However, we can express this form as $M_{(1,2,2,2),(1,1,1,1),-2}$. 


\section{$3.19(1,2,2,3)$}

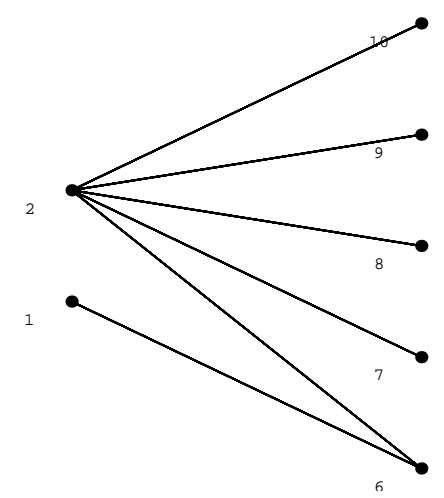

A representative equation for this family of hypersurfaces is $x^{8}+y^{4}+z^{4}+x^{2} w^{2}+$ $y w^{2}+z w^{2}$.

The Fibration. $\rho=7$ and no curves have genus 1. We will see curves 2, 7-10 as a $\tilde{D}_{4}$ so that curve 6 is a 2 -section and curve 1 is part of an $\tilde{A}_{1}$. The corresponding Jacobian fibration has good rank.

Method: Mukai and Matrix. We may apply Mukai's theorem to see that $\operatorname{Pic}(S)$ is of index 2 in $\operatorname{Pic}(\mathbf{J}(S))$, so that $\operatorname{disc} \operatorname{Pic}(S)=4 \cdot \operatorname{Pic}(\mathbf{J}(S))$ and the intersection matrix for our graph determines $\operatorname{Pic}(S)$. The discriminant of the matrix is 32 . We have generators of orders 2,4 , and 8. Thus the discriminant group is either $\mathbb{Z}_{8} \oplus \mathbb{Z}_{4}$ or $\mathbb{Z}_{8} \oplus \mathbb{Z}_{2} \oplus \mathbb{Z}_{2}$.

We need to embed the form in $v \perp w_{2,1}^{-1}$; examine the forms on $\mathbb{Z}_{8} \cdot w_{2,3}^{1}$ and $w_{2,3}^{5}$ reduce to $w_{2,1}^{1}$; in order obtain something isomorphic to $v \perp w_{2,1}^{-1}$, the remaining part must be $\left(w_{2,1}^{1}\right)^{2} . w_{2,3}^{-1}$ and $w_{2,3}^{-5}$ reduce to $w_{2,1}^{-1}$; the remaining part could only be $v$. So we must determine which of the four possible forms we have. We have two 7/8-valued generators which are dependent; what sum of generators could this be? We can have $1 / 8+\{1 / 2,1\}, 5 / 8+\{1 / 2,1\}, 13 / 8+\{0,1\}, \& 15 / 8+\{0,1\}$. The only combination which gives $7 / 8$ is $15 / 8+1$, so $\operatorname{Pic}(S)=w_{2,3}^{-1} \perp v$. This lattice has no representation as a sum of Dynkin lattices. However, we can express this form as 
$M_{(1,1,1,1,2),(1,1,1,1,1),-2}$.

\section{$3.20(1,6,8,9)$}

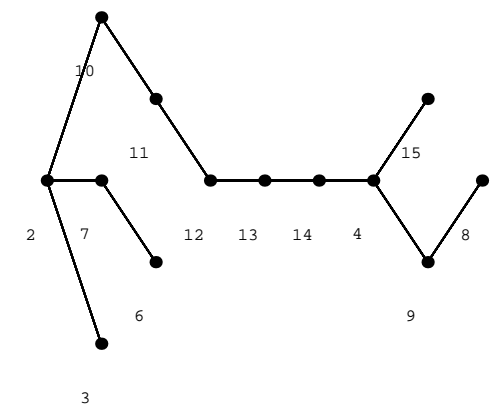

A representative equation for this family of hypersurfaces is $x^{24}+y^{4}+z^{3}+x^{6} w^{2}+y w^{2}$. This is one of Arnold's 14 original surface singularities. $\rho=12$ and curve 3 has genus 1. If we consider curve 3 to be a fibre, then curve 2 is a section and we are left with $\tilde{E}_{8}$ and $\tilde{A}_{2}$. We assume that the fibre must be completed. This fibration has good rank and the associated form has no isotropic subgroups, so $\operatorname{Pic}(S)=E_{8} \perp A_{2} \perp U$.

\section{$3.21(1,1,1,2)$}

A representative equation for this family of hypersurfaces is $x^{5}+y^{5}+z^{5}+x w^{2}+$ $y w^{2}+z w^{2}$ $\rho=2$ and we will go directly to the matrix as there is no graph. The matrix is

$$
\left(\begin{array}{ccccc}
2 & 2 & 1 & 5 & 2 \\
2 & 2 & 1 & 5 & 2 \\
1 & 1 & -2 & 0 & 1 \\
5 & 5 & 0 & 10 & 5 \\
2 & 2 & 1 & 5 & 2
\end{array}\right)
$$


which easily reduces to $\left(\begin{array}{cc}2 & 1 \\ -1 & 2\end{array}\right)$ which corresponds to the form $w_{5,1}^{-1}$. We will continue to specify it by the simplified matrix, and it has no representation as a sum of Dynkin lattices.

\section{$3.22(1,3,5,6)$}

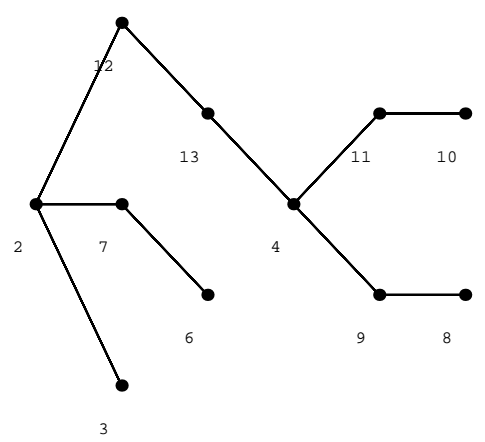

A representative equation for this family of hypersurfaces is $-x^{15}+y^{5}+z^{3}+x^{3} w^{2}+$ $y w^{2}$.

This is one of Arnold's 14 original surface singularities. $\rho=10$ and curve 3 has genus 1 . If we consider curve 3 to be a fibre, then curve 2 is a section and we are left with $\tilde{E}_{6}$ and $\tilde{A}_{2}$. We assume that the fibre must be completed. This fibration has good rank, but the associated form has an isotropic subgroup. There are at most 3 sections because the discriminant groups on $\tilde{E}_{6}$ and $\tilde{A}_{2}$ is $\mathbb{Z}_{3}$. If there are 3 sections, then $\operatorname{disc}(\operatorname{Pic}(S))=1$; the only unimodular lattice of rank 10 is $E_{8} \perp U$, but this only has one section. Contradiction! If there are 2 sections, then $4 \cdot \operatorname{disc}(\operatorname{Pic}(S))=9$ and $\operatorname{disc}(\operatorname{Pic}(S))$ is not an integer; contradiction. Therefore our fibration has only one section (which we have exhibited), so $\operatorname{Pic}(S)=E_{6} \perp A_{2} \perp U$. 


\section{$3.23(2,2,3,5)$}

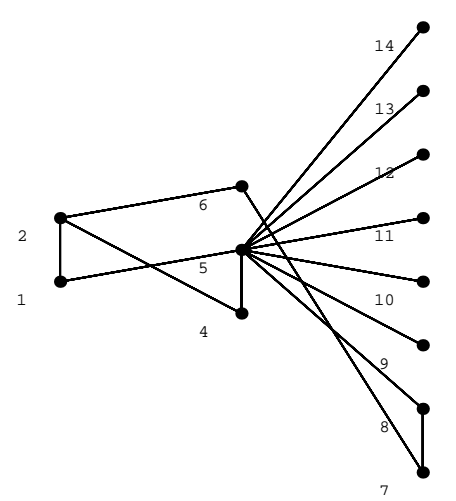

A representative equation for this family of hypersurfaces is $x^{6}+y^{6}+z^{4}+x w^{2}+y w^{2}$. The Fibration. $\rho=11$ and curves 1 and 4 have genus 1 . If we consider these as fibres, then curves 2 and 5 are sections and we may complete each of curves 9-14 to an $\tilde{A}_{1}$ and curves $6,7,8$ to an $\tilde{A}_{3}$. This fibration has good rank but we have exhibited two sections.

Method: Intermediate Lattice Calculation. Our discriminant group is $\mathbb{Z}_{4} \oplus$ $\left(\mathbb{Z}_{2}\right)^{6}$. The last six entries can be permuted without harm, so up to this permutation we have the following possible isotropic subgroup generators: $(0,0,0,1,1,1,1)$, $(2,1,1,0,0,0,0)$, and $(2,1,1,1,1,1,1)$.

We begin with $(0,0,0,1,1,1,1)$. Our initial discriminant form is $w_{2,2}^{5} \perp\left(w_{2,1}^{-1}\right)^{6}$; we will suppress the three empty entries in our calculation. Up to permutation, we have the following elements and values:

$\begin{array}{llll}0000 & 0 & 1111 & 0 \\ 0001 & -1 / 2 & 0111 & -3 / 2 \\ 0011 & -1 & 0011 & -1\end{array}$

We have 8 elements (no longer up to permutation), arranged by coset of $(1,1,1,1)$ : 


$\begin{array}{lll}0000 & 1111 & 0 \\ 0011 & 1100 & -1 \\ 0101 & 1010 & -1 \\ 1001 & 0110 & -1\end{array}$

This corresponds to the form $v$ so we have $v \perp w_{2,2}^{5} \perp\left(w_{2,1}^{-1}\right)^{2}$.

Now , using the same procedure, we examine $(2,1,1,0,0,0,0)$.

$\begin{array}{llllllll}000 & 0 & 211 & 0 & 100 & 5 / 4 & 311 & 1 / 4 \\ 001 & -1 / 2 & 210 & 1 / 2 & 101 & 3 / 4 & 310 & 3 / 4 \\ 011 & -1 & 200 & 1 & 111 & 1 / 4 & 300 & 5 / 4\end{array}$

So in $H^{\perp}$ we have, arranged by coset,

$$
\begin{array}{lll}
000 & 211 & 0 \\
011 & 200 & 1 \\
101 & 310 & 3 / 4 \\
110 & 301 & 3 / 4
\end{array}
$$

which corresponds to $w_{2,2}^{-5}$, so we have $w_{2,2}^{-5} \perp\left(w_{2,1}^{-1}\right)^{4}$.

Now, using the same procedure, we do $(2,1,1,1,1,1,1)$.

$\begin{array}{llllllll}0000000 & 0 & 2111111 & 0 & 1000000 & 5 / 4 & 3111111 & 1 / 4 \\ 0000001 & -1 / 2 & 2111110 & 1 / 2 & 1000001 & 3 / 4 & 3111110 & 3 / 4 \\ 0000011 & -1 & 2111100 & -1 & 1000011 & 1 / 4 & 3111100 & 5 / 4 \\ 0000111 & 1 / 2 & 2111000 & -1 / 2 & 1000111 & -1 / 4 & 3111000 & 7 / 4 \\ 0001111 & 0 & 2110000 & 0 & 1001111 & 5 / 4 & 3110000 & 1 / 4 \\ 0011111 & -1 / 2 & 2100000 & 1 / 2 & 1011111 & 3 / 4 & 3100000 & 3 / 4 \\ 0111111 & -1 & 2000000 & 1 & 1111111 & 1 / 4 & 3000000 & 5 / 4\end{array}$

so in $H^{\perp}$ we have, arranged by coset of $H$, 


$\begin{array}{llll}\text { element } & \text { elt. }+H & \text { value } & \text { no. of elts. } \\ 0000000 & 2111111 & 0 & 1 \\ 0000011 & 2111100 & -1 & 15 \\ 0001111 & 2110000 & 0 & 15 \\ 0111111 & 2000000 & 1 & 1 \\ 1000001 & 3111110 & 3 / 4 & 6 \\ 1000111 & 3111000 & 7 / 4 & 20 \\ 1011111 & 3100000 & 3 / 4 & 6\end{array}$

There are elements of order 4 , so the discriminant group is $\mathbb{Z}_{4} \oplus\left(\mathbb{Z}_{2}\right)^{4}$. We can eliminate all forms which involve $w_{2,1}^{ \pm 1}$ as there would be elements with value $1 / 2$ or $-1 / 2$. This leaves us with $\left\{w_{2,2}^{-1}\right.$ or $\left.w_{2,2}^{-5}\right\} \perp\{u \perp u \cong v \perp v$ or $u \perp v\}$. Checking the values for each of these possibilities shows that the form must be $w_{2,2}^{-5} \perp u \perp v \cong$ $w_{2,2}^{-1} \perp v \perp v$.

Now we need to determine which lattice is the correct one. The only way to do this is to (a) exhibit a fibration corresponding to one of the forms and (b) show that none of the other lattices can be exhibited as a fibration. There are three possibilities for a fibration here: $\left(A_{1}\right)^{2} \perp D_{4} \perp A_{3} \perp U,\left(A_{1}\right)^{4} \perp D_{5} \perp U$, and $D_{5} \perp D_{4} \perp U(2)$. With much pain, one may show that the first two of these are not possible, no matter what kind of curves are added. We may see the last one in the following way.

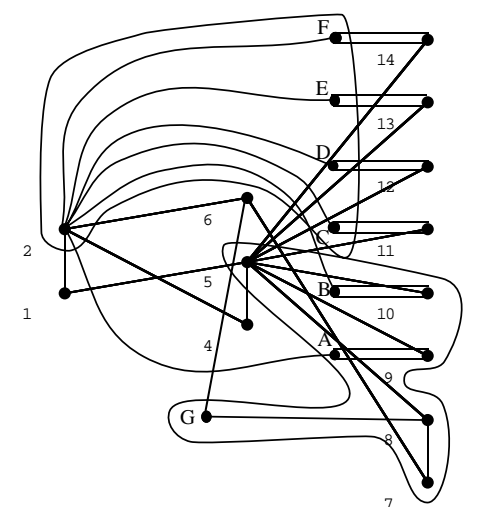


Referring to our original fibration with the $6 \tilde{A}_{1}$, name the curves attached to curves $9-14$ by A - F, and the curve attached to curves 6 and 8 by G. Then, we may view curves 7 - 10, G as forming a $\tilde{D}_{5}$, so that curves 1, 4, 6, 11 - 14, A, B are 2-sections, and curves $2, \mathrm{C}$ - F form a $\tilde{D}_{4}$.

Thus, $\operatorname{Pic}(S)=D_{5} \perp D_{4} \perp U(2)$.

\section{$3.24(1,2,4,5)$}

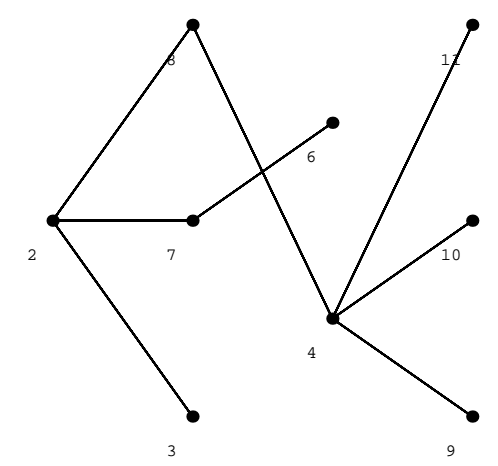

A representative equation for this family of hypersurfaces is $x^{12}+y^{6}+z^{3}+x^{2} w^{2}+y w^{2}$. $\rho=8$ and curve 3 has genus 1 . If we consider it as a fibre, then curve 2 is a section and we are left with curves $4,8-11$ as a $\tilde{D}_{4}$ and we can complete curves 7,8 to $\tilde{A}_{2}$. This fibration has good rank. The discriminant form is $v \perp w_{3,1}^{1}$, which has no isotropic subgroups. Therefore, $\operatorname{Pic}(S)=D_{4} \perp A_{2} \perp U$. 


\section{$3.25(1,1,3,4)$}

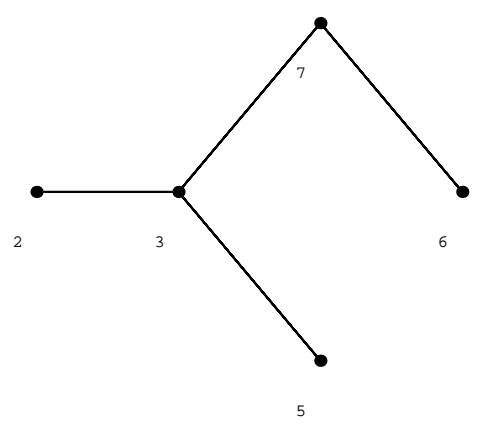

A representative equation for this family of hypersurfaces is $-x^{9}+y^{9}+z^{3}+x w^{2}+y w^{2}$. $\rho=4$ and curves 5 and 2 have genus 1 . If we consider these as fibres, then curve 3 is a section and we may complete the remaining curves 6,7 to an $\tilde{A}_{2}$. This fibration has good rank, and the associated form $w_{3,1}^{1}$ has no isotropic subgroups, $\operatorname{so} \operatorname{Pic}(S)=$ $A_{2} \perp U$.

\section{$3.26 \quad(2,4,5,9)$}

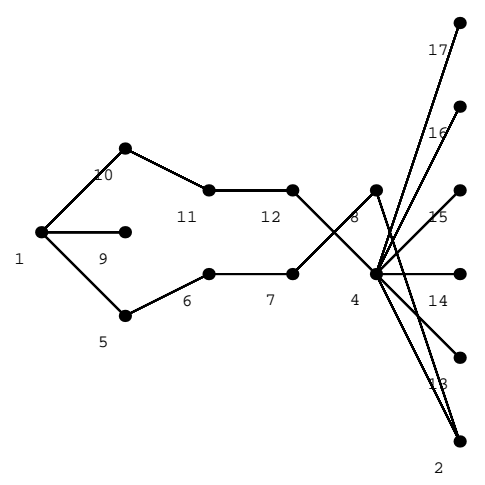

A representative equation for this family of hypersurfaces is $x^{10}+y^{5}+z^{4}+x w^{2}$.

The Fibration. $\rho=14$ and curve 2 has genus 1. If we consider it to be a fibre, then curves 4 and 8 are sections and curves 1, 5-7, 9-12 form a $\tilde{E}_{7}$. The remaining curves 13-17 can each be completed to an $\tilde{A}_{1}$. This fibration has good rank and we 
have exhibited two sections.

Method: Intermediate Lattice Calculation. Each of these fibres has discriminant group $Z_{2}$, so we begin with $\left(Z_{2}\right)^{6}$. The quadratic form on the generator for $E_{7}$ evaluates to $-3 / 4$ and on the generator for $A_{1}$ evaluates to $-1 / 4$ (using Miranda's notation). Therefore, the three distinct isotropic subgroups are generated by $(1,1,0,0,0,0)$, $(0,0,1,1,1,1)$, and $(1,1,1,1,1,1)$.

Begin with $(1,1,0,0,0,0)$. We suppress the last four entries. There are only four elements: $(0,0),(0,1),(1,0),(1,1)$.

We determine which of these are perpendicular to $H=(1,1)$ with respect to the quadratic form. This is true for $a$ when $q(a)-q(a+(1,1))=0$; we reverse the order because we only care about the magnitude:

$(0,0)-0-0=0 ;(0,1)-1 / 4-3 / 4=-1 / 2$

$(1,0)-3 / 4-1 / 4=1 / 2 ;(1,1)-0-0=0$

This leaves us with $(0,0)$ and $(1,1)$ which are congruent under $H$, so we are left with the identity group and so the form is $\left(w_{2,1}^{-1}\right)^{4}$.

On to $(0,0,1,1,1,1)$. Up to permutation of the entries, we have 5 elements:

$$
(0,0,0,0,0,0),(0,0,0,0,0,1),(0,0,0,0,1,1),(0,0,0,1,1,1),(0,0,1,1,1,1)
$$

We will suppress the first 2 entries. Now we determine which of these are perpendicular to $H=(1,1,1,1)$ with respect to the quadratic form. Respectively, for these 5 types of elements, we get: $(0,0,0,0,0,0)-0-0=0$

$$
\begin{aligned}
& (0,0,0,0,0,1)--1 / 4-(-3 / 4)=1 / 2 ;(0,0,0,0,1,1)--2 / 4-(-2 / 4)=0 \\
& (0,0,0,1,1,1)--3 / 4-(-1 / 4)=-1 / 2 ;(0,0,1,1,1,1)-0-0=0
\end{aligned}
$$

and can then list all elements in $H^{\perp}$ by coset of $H$ : 


$$
\begin{array}{ll}
(0,0,0,0) & (1,1,1,1) \\
(0,0,1,1) & (1,1,0,0) \\
(0,1,0,1) & (1,0,1,0) \\
(0,1,1,0) & (1,0,0,1)
\end{array}
$$

These determine the form $v$. We retain $w_{2,1}^{-1} \perp w_{2,1}^{1}$ from the suppressed entries.

Now for $(1,1,1,1,1,1)$. This time, up to permutation, we have twelve elements: $(0,0,0,0,0,0),(0,0,0,0,0,1),(0,0,0,0,1,1),(0,0,0,1,1,1),(0,0,1,1,1,1),(0,1,1,1,1,1)$, $(1,0,0,0,0,0),(1,0,0,0,0,1),(1,0,0,0,1,1),(1,0,0,1,1,1),(1,0,1,1,1,1),(1,1,1,1,1,1)$. Now we determine which of these are in $H^{\perp}$ :

$$
\begin{aligned}
& (0,0,0,0,0,0)-0-0=0 ;(0,0,0,0,0,1)-1 / 4-3 / 4=-1 / 2 \\
& (0,0,0,0,1,1)-2 / 4-2 / 4=0 ;(0,0,0,1,1,1)-3 / 4-1 / 4=1 / 2 \\
& (0,0,1,1,1,1)-0-0=0 ;(0,1,1,1,1,1)-1 / 4-3 / 4=1 / 2 \\
& (1,0,0,0,0,0)-3 / 4-1 / 4=1 / 2 ;(1,0,0,0,0,1)-0-0=0 \\
& (1,0,0,0,1,1)-1 / 4-3 / 4=0 ;(1,0,0,1,1,1)-2 / 4-2 / 4=0 \\
& (1,0,1,1,1,1)-3 / 4-1 / 4=0 ;(1,1,1,1,1,1)-0-0=0
\end{aligned}
$$

So, perpendicular to $(1,1,1,1,1,1)$ and arranged by coset, we have:

$$
\begin{array}{ll}
(0,0,0,0,0,0) & (1,1,1,1,1,1) \\
(0,0,0,0,1,1) & (1,0,0,1,1,1) \\
(0,0,1,1,1,1) & (1,0,0,0,0,1)
\end{array}
$$

Counting the elements shows that there are 10 elements with value $1 / 2$ and 6 with value 0. Every element is of order 2, so a simple check shows that these values correspond to $u \perp v$.

Now we need to determine which of the $H$ is the correct one. The only way to do this is to (a) exhibit a fibration corresponding to one of the forms and (b) show that none of the other lattices can be exhibited as a fibration. There are three possibilities 
for a fibration here: $E_{8} \perp\left(A_{1}\right)^{4} \perp U, E_{7} \perp D_{4} \perp A_{1} \perp U$, and $D_{4} \perp D_{8} \perp U$. With much pain, one may show that the first two of these are not possible, no matter what kind of curves are added. We may see the last one in the following way.

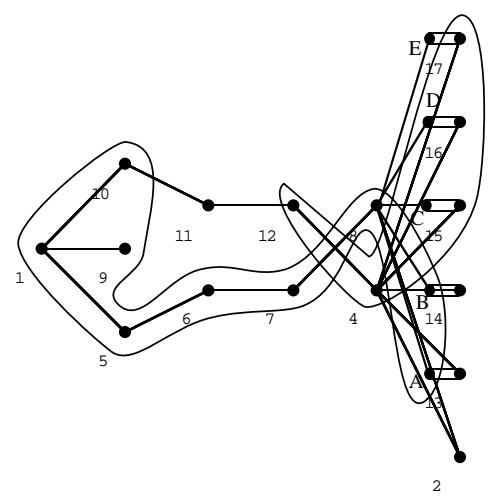

Referring to our original fibration with the $5 \tilde{A}_{1}$, name the curves attached to curves 13 - 17 by A - E. Then we can see curves $4,12,15-17$ as forming a $\tilde{D}_{4}$, so that curve 11 is a section and curves $1,13,14, \mathrm{C}$ - E are 2-sections. Then curves 1, 5 10, A, B form a $\tilde{D}_{8}$. Thus, $\operatorname{Pic}(S)=D_{8} \perp D_{4} \perp U$.

\section{$3.27(2,3,8,11)$}

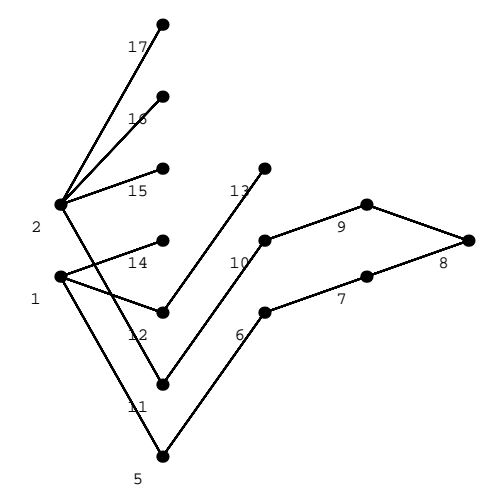

A representative equation for this family of hypersurfaces is $x^{12}+y^{8}+z^{3}+x w^{2}$.

$\rho=14$. None of the curves have genus 1 , so we will see an $\tilde{E}_{8}$ composed of curves 1, $5-9,12-14$. Then curve 10 is a section and we curves $2,11,15-17$ form a $\tilde{D}_{4}$. This fibration has good rank, so $\operatorname{Pic}(S)=E_{8} \perp D_{4} \perp U$. 


\section{$3.28(1,3,7,10)$}

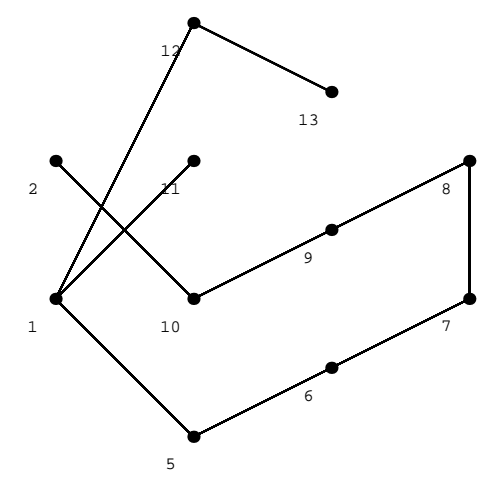

A representative equation for this family of hypersurfaces is $x^{21}+y^{7}+z^{3}+x w^{2}$.

$\rho=10$ and curve 2 has genus 1 . If we consider it as a fibre, then curve 10 is a section, leaving curves 1, 5-9, 11-13 forming an $\tilde{E}_{8}$. This fibration has good rank, and has trivial discriminant group, so $\operatorname{Pic}(S)=E_{8} \perp U$.

\section{$3.29(4,5,6,15)$}

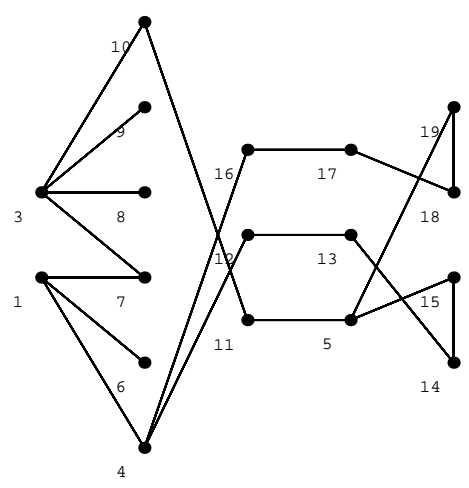

A representative equation for this family of hypersurfaces is $x^{5} y^{2}+x^{6} z+y^{6}+z^{5}+w^{2}$.

The Fibration. $\rho=16$. None of the curves has genus 1. The easiest fibre to see is an $\tilde{A}_{9}$ formed by curves $4,5,12-19$, so that curves 1 and 11 are sections, which leaves curves $3,7-10$ forming a $\tilde{D}_{4}$ and we may complete curve 6 to an $\tilde{A}_{1}$. This fibration has good rank. 
Method: Intermediate Lattice Calculation. Using Miranda's mod $\mathbb{Z}$ convention, the only distinct isotropic subgroup is generated by $(5,(1,1), 1)$. We list elements and their values with a view to determine which are in $H^{\perp}$.

\begin{tabular}{|c|c|c|c|c|c|c|c|}
\hline \multicolumn{2}{|c|}{$\begin{array}{lllll}0 & 0 & 0 & 0\end{array}$} & 5111 & 10 & 0100 & $-1 / 2$ & \multicolumn{2}{|c|}{$\begin{array}{llll}5 & 011 & 0\end{array}$} \\
\hline & 01 & 511 & $-3 / 4$ & 0101 & $-3 / 4$ & 5010 & $-3 / 4$ \\
\hline & 10 & 510 & 0 & 0110 & $-1 / 2$ & 5001 & $-1 / 2$ \\
\hline & 11 & 510 & $-3 / 4$ & 0111 & $-3 / 4$ & 5000 & $-1 / 4$ \\
\hline 1000 & $-9 / 20$ & 6111 & $-19 / 20$ & 1100 & $-19 / 20$ & 6011 & $-19 / 20$ \\
\hline 1001 & $-14 / 20$ & 6110 & $-14 / 20$ & 1101 & $-4 / 20$ & 6010 & $-14 / 20$ \\
\hline 1010 & $-19 / 20$ & 6101 & $-19 / 20$ & 1110 & $-19 / 20$ & 6001 & $-9 / 20$ \\
\hline 1011 & $-4 / 20$ & 6100 & $-14 / 20$ & 1111 & $-4 / 20$ & 6000 & $-4 / 20$ \\
\hline 2000 & $-4 / 5$ & 7111 & $-4 / 5$ & 2100 & $-6 / 20$ & 7011 & $-4 / 5$ \\
\hline 2001 & $-1 / 20$ & 7110 & $-11 / 20$ & 2101 & $-11 / 20$ & 7010 & $-11 / 20$ \\
\hline 2010 & $-6 / 20$ & 7101 & $-4 / 5$ & 2110 & $-6 / 20$ & 7001 & $-6 / 20$ \\
\hline 2011 & $-11 / 20$ & 7100 & $-11 / 20$ & 2111 & $-11 / 20$ & 7000 & $-1 / 20$ \\
\hline 3000 & $-1 / 20$ & 8111 & $-11 / 20$ & 3100 & $-11 / 20$ & 8011 & $-11 / 20$ \\
\hline 3001 & $-6 / 20$ & 8110 & $-6 / 20$ & 3101 & $-4 / 5$ & 8010 & $-6 / 20$ \\
\hline 3010 & $-11 / 20$ & 8101 & $-11 / 20$ & 3110 & $-11 / 20$ & 8001 & $-1 / 20$ \\
\hline 3011 & $-4 / 5$ & 8100 & $-6 / 20$ & 3111 & $-4 / 5$ & 8000 & $-4 / 5$ \\
\hline 4000 & $-1 / 5$ & 9111 & $-4 / 20$ & 4100 & $-14 / 20$ & 9011 & $-4 / 20$ \\
\hline 4001 & $-9 / 20$ & 9110 & $-19 / 20$ & 4101 & $-19 / 20$ & 9010 & $-19 / 20$ \\
\hline 4010 & $-14 / 20$ & 9101 & $-4 / 20$ & 4110 & $-14 / 20$ & 9001 & $-14 / 20$ \\
\hline 4011 & $-19 / 20$ & 9100 & $-19 / 20$ & 4111 & $-9 / 20$ & 9000 & $-9 / 20$ \\
\hline
\end{tabular}

Here is a table of members of $H^{\perp}$ arranged by coset of $H$. 


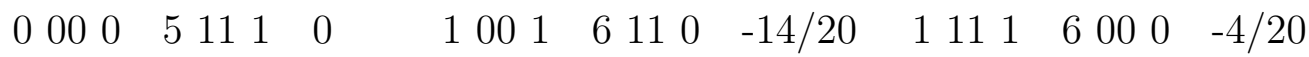

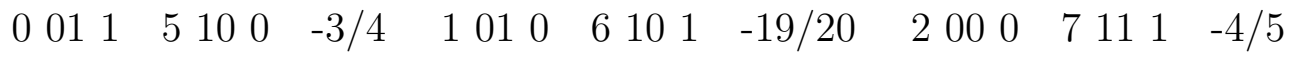

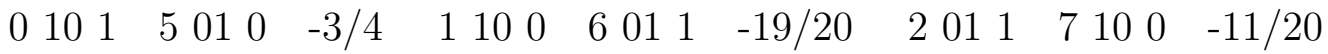

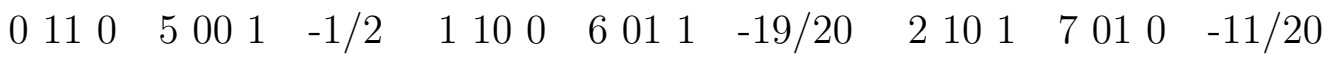

$$
\begin{aligned}
& 2110 \quad 7001 \quad-6 / 20 \quad 4011 \quad 9100 \quad-19 / 20 \\
& 3001 \quad 8110 \quad-6 / 20 \quad 4101 \quad 90100 \quad-19 / 20
\end{aligned}
$$

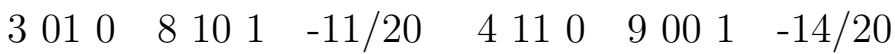

$$
\begin{aligned}
& \begin{array}{llllllllllll}
311 & 8 & 00 & 0 & -4 / 5 & 411 & 9 & 00 & 0 & -9 / 20
\end{array}
\end{aligned}
$$

It appears that we have two distinct subgroups of order 2 generated by 0011 and 0101 with value $-3 / 4$, and a subgroup of order 5 generated by 2000 with value $-4 / 5$. These generate a group of order 20 (which we have), so we have the form $w_{5,1}^{-1} \perp\left(w_{2,1}^{1}\right)^{2}$, and so $\operatorname{Pic}(S)=T_{2,5,5} \perp D_{6}$.

\section{$3.30 \quad(5,7,8,20)$}

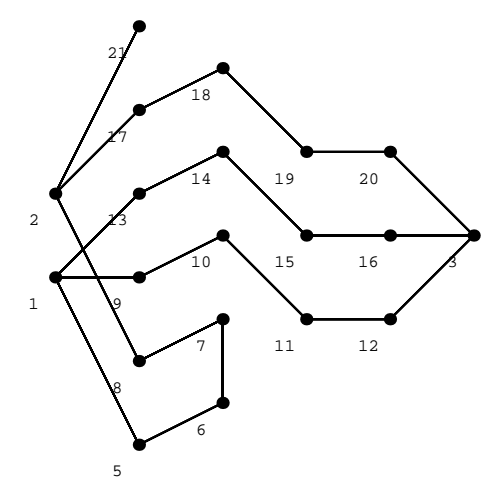

A representative equation for this family of hypersurfaces is $x^{8}+x y^{5}+z^{5}+w^{2}$.

The Fibration. $\rho=18$ and no curves have genus 1 . The easiest fibration to see is to combine curves $1,3,9-16$ into a $\tilde{A}_{9}$ and curves $2,6-8,17-19,21$ into a $\tilde{E}_{7}$, which leaves curves 5 and 20 as sections. This fibration has good rank. 
Method: Intermediate Lattice Calculation. Using Miranda's mod $\mathbb{Z}$ convention, the only isotropic subgroup $H$ is generated by $(5,1)$, and here is a table of elements and values so that we can determine which elements are in $H^{\perp}$.
$(0,0) \quad 0$
$(5,1) \quad 0$
$(0,1) \quad-3 / 4$
$(5,0) \quad-1 / 4$
$(3,0) \quad-1 / 20$
$(7,0) \quad-1 / 20$
$\begin{array}{llll}(1,0) & -9 / 20 \quad(6,1) & -19 / 20\end{array}$
$(3,1) \quad-4 / 5$
$(8,1) \quad-11 / 20$
$\begin{array}{llll}(1,1) & -1 / 5 & (6,0) & -1 / 5\end{array}$
$(4,0) \quad-1 / 5$
$(8,0) \quad-4 / 5$
$(2,0) \quad-4 / 5$
$(7,1) \quad-4 / 5$
$(4,1) \quad-19 / 20$
$(9,1) \quad-1 / 5$

Now we list the elements of $H^{\perp}$ arranged by coset of $H$ so we can determine our form.

$$
\begin{array}{lll}
(0,0) & (5,1) & 0 \\
(1,1) & (6,0) & -1 / 5 \\
(2,0) & (7,1) & -4 / 5 \\
(3,1) & (8,0) & -4 / 5 \\
(4,0) & (9,1) & -1 / 5
\end{array}
$$

This corresponds to the form $w_{5,1}^{-1}$, so $\operatorname{Pic}(S)=E_{8} \perp T_{2,5,5}$.

\section{$3.31(3,4,5,12)$}

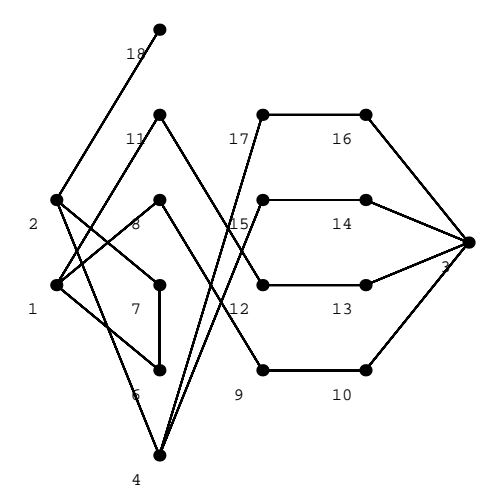


A representative equation for this family of hypersurfaces is $x^{8}+y^{6}+x^{3} z^{3}+y z^{4}+w^{2}$. The Fibration. $\rho=15$ and none of the curves have genus 1 . There is no Jacobian fibration of good rank. Instead, we find a non-Jacobian fibration whose Jacobian fibration has good rank: curves 1, 6, 8-13 form an $\tilde{E}_{7}$ and curves 2, 4, 14-18 form an $\tilde{E}_{6}$, leaving curves 3 and 7 as 2 -sections.

Method: Mukai and Matrix. We apply Mukai's theorem to see that $\operatorname{Pic}(S)$ is of index 2 in $\operatorname{Pic}(\mathbf{J}(S))$, so that $\operatorname{disc} \operatorname{Pic}(S)=4 \cdot \operatorname{Pic}(\mathbf{J}(S))$ and the intersection matrix for our graph determines $\operatorname{Pic}(S)$. The matrix has discriminant 24. We have a 1/8-valued generator of order 8 and the $2 / 3$-valued generator of order 3 which are independent, so our form is $w_{2,3}^{1} \perp w_{3,1}^{-1}$. This does, in fact, embed into $w_{2,1}^{1} \perp w_{3,1}^{-1}$ (see 3.19), and so $\operatorname{Pic}(S)=E_{6} \perp A_{7} \perp U$.

\section{$3.32(2,2,3,7)$}

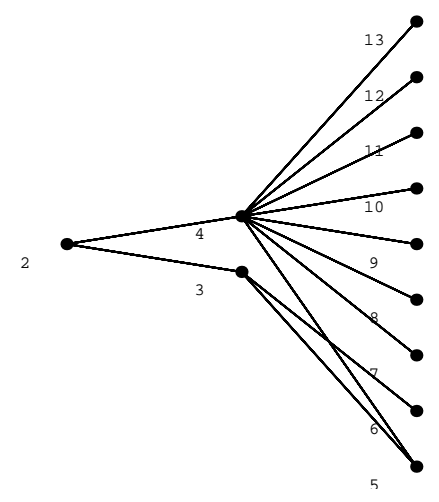

A representative equation for this family of hypersurfaces is $-x^{7}+y^{7}+x z^{4}+y z^{4}+w^{2}$.

The Fibration. $\rho=10$ and curves 2 and 5 are of genus 1 . if we consider them to be fibres, then curves 3 and 4 are sections and we may complete each of curves 6-13 to a $\tilde{A}_{1}$. The fibration $8 \tilde{A}_{1}$ has good rank.

Method: Intermediate Lattice Calculation. The only two $q$-isotropic subgroups 
(up to permutation) are generated by $(1,1,1,1,0,0,0,0)$ and $(1,1,1,1,1,1,1,1)$.

For $(1,1,1,1,0,0,0,0)$, we suppress the last four zeros. Up to permutation, we have the following elements and values: $(0,0,0,0)-0,(0,0,0,1)--1 / 4,(0,0,1,1)--1 / 2,(0,1,1,1)$ $--3 / 4$, and $(1,1,1,1)-0$. We evaluate these as to whether they are perpendicular to $(1,1,1,1)$ and list them, as usual, by coset:

$$
\begin{array}{lll}
(0,0,0,0) & (1,1,1,1) & \text { value is } 0 \\
(0,0,1,1) & (1,1,0,0) & \text { value is }-1 / 2 \\
(0,1,0,1) & (1,0,1,0) & \text { value is }-1 / 2 \\
(0,1,1,0) & (1,0,0,1) & \text { value is }-1 / 2
\end{array}
$$

This form corresponds to $v$, so we have $v \perp\left(w_{2,1}^{-1}\right)^{4}$.

For $(1,1,1,1,1,1,1,1)$, up to permutation we have the following elements and values:

$$
\begin{array}{llll}
(0,0,0,0,0,0,0,0) & 0 & (0,0,0,0,1,1,1,1) & 0 \\
(0,0,0,0,0,0,0,1) & -1 / 4 & (0,0,0,1,1,1,1,1) & -1 / 4 \\
& & (0,0,1,1,1,1,1,1) & -1 / 2 \\
(0,0,0,0,0,0,1,1) & -1 / 2 & (0,1,1,1,1,1,1,1) & -3 / 4 \\
(0,0,0,0,0,1,1,1) & -3 / 4 & (1,1,1,1,1,1,1,1) & 0
\end{array}
$$

This leaves us with the following types of elements:

$$
\begin{array}{llll}
(0,0,0,0,0,0,0,0) & (1,1,1,1,1,1,1,1) & \text { value } 0 & 1 \text { element } \\
(0,0,0,0,0,0,1,1) & (0,0,1,1,1,1,1,1) & \text { value }-1 / 2 & \left(\begin{array}{l}
8 \\
2
\end{array}\right)=28 \text { elements } \\
& & & \\
(0,0,0,0,1,1,1,1) & (0,0,0,0,1,1,1,1) & \text { value } 0 & \left(\begin{array}{l}
8 \\
4
\end{array}\right) / 2=35 \text { elements }
\end{array}
$$

So, what form is this? Examining App. 1, we see that we have $u \perp u \perp u$.

Now we need to determine which of the $H$ is the correct one. The only way to do this is to (a) exhibit a fibration corresponding to one of the forms and (b) show that none of the other lattices can be exhibited as a fibration. There are two possibilities for a fibration here: $D_{4} \perp\left(A_{1}\right)^{4} \perp U$ and $D_{4} \perp D_{4} \perp U(2)$. With much pain, one 
may show that the first is not possible, no matter what kind of curves are added. We may see the last one in the following way.

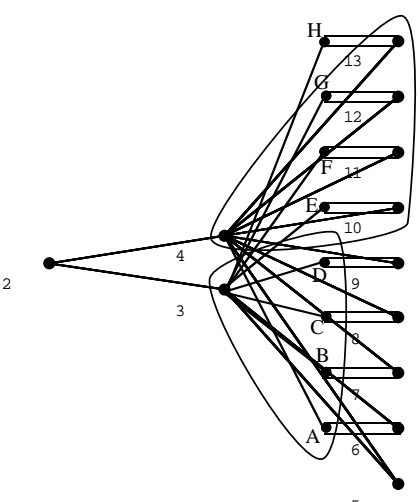

Referring to our original fibration with the $8 \tilde{A}_{1}$, name the curves attached to curves 6 - 13 by A - H. Then we may see curves 4, 10 - 13 as a $\tilde{D}_{4}$ so that curves E - H, A, 7 - 9 are 2-sections, and curves $3,6, \mathrm{~B}$ - D form the other $\tilde{D}_{4}$.

Thus, $\operatorname{Pic}(S)=D_{4} \perp D_{4} \perp U(2)$.

\section{$3.33(2,3,4,9)$}

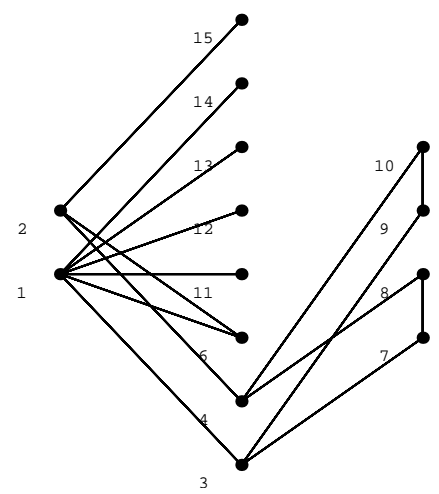

A representative equation for this family of hypersurfaces is $x^{9}+y^{6}+x z^{4}+y^{2} z^{3}+w^{2}$.

The Fibration. $\rho=12$ and curve 3 has genus 1 . The fibration will be non-Jacobian but its Jacobian fibration has good rank. There is only one such fibration which intersects with curve 5 correctly; namely, consider curve 3 as a 2-section, and see curves $1,11-14$ as a $\tilde{D}_{4}$ and curves $2,4,7-10,15$ as an $\tilde{E}_{6}$, leaving curve 6 as another 
2-section.

Method: Mukai and Matrix. We can apply Mukai's theorem to see that $\operatorname{Pic}(S)$ is of index 2 in $\operatorname{Pic}(\mathbf{J}(S))$, so that $\operatorname{disc} \operatorname{Pic}(S)=4 \cdot \operatorname{Pic}(\mathbf{J}(S))$ and the intersection matrix for our graph determines $\operatorname{Pic}(S)$. The matrix has discriminant 48 . We have two of the 1-valued generators which are independent of a 2/3-valued generator of order 3 , and the sum of the two 1-valued generators has value 1, which indicates that we have $w_{3,1}^{-1} \perp v$. To complete the calculation, we examine some $2 / 3$-valued generators of order 6 . The sum of two of them has value $2 / 3$, indicating that the order-3 portion of each represents the same element of $\mathbb{Z}_{3}$, and that the order-2 portions each have value 0 and sum-value 0 . The only order- 2 form values that exist which sum to 0 are 0 from $u, 1+1$ where one is from $u$ and one is from $v$ (we can't have two $u$ and $v \perp v$ won't reduce to $v$ ), or $1 / 2+3 / 2$ where the $1 / 2$ is from $w_{2,1}^{1}$ and the $3 / 2$ is from $w_{2,1}^{-1}$. So our possibilities for the sum of the order- 2 portions of these two generators are $0+0=1$ in $u, 1+1+1+1=1(1+1$ in $u=0,1+1$ in $v=1)$,

and $1 / 2+3 / 2+1 / 2+3 / 2=0$. So our form must be $w_{3,1}^{-1} \perp v \perp w_{2,1}^{1} \perp w_{2,1}^{-1}$. This lattice has no representation as a sum of Dynkin lattices. However, we can express this form as $M_{(1,1,1,1,2,2,3),(1,1,1,1,1,1,1),-4}$.

\section{$3.34(2,6,7,15)$}

A representative equation for this family of hypersurfaces is $x^{15}+y^{5}+x z^{4}+w^{2}$. See (3.26). They have the same rank and configuration of curves. 


\section{$3.35(3,4,7,14)$}

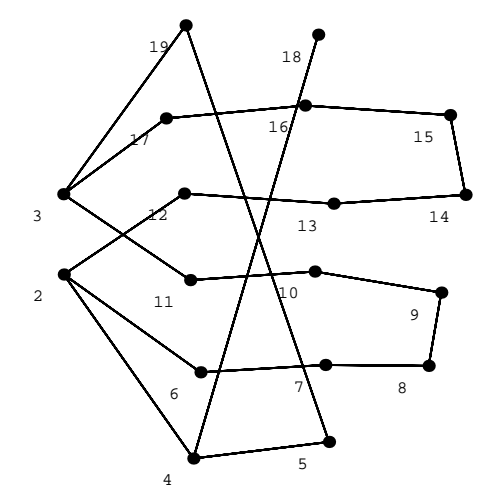

A representative equation for this family of hypersurfaces is $x^{8} y+x^{7} z+y^{7}+z^{4}+w^{2}$. The Fibration. $\rho=16$ and curve 5 has genus 1. If we consider curve 5 to be a fibre, then curves 4 and 19 must be sections. We are left with curves $2,3,6-17$ forming an $\tilde{A}_{13}$ and we may complete curve 18 to be an $\tilde{A}_{1}$. This fibration has good rank; we have exhibited 2 sections.

Method: Intermediate Lattice Calculation. We use Miranda's notation. The only element in $\mathbb{Z}_{14}$ with a value of $-3 / 4$ is 7 , so our only isotropic subgroup is of order 2 and generated by $(7,1)$. We have 28 elements and corresponding values:

$$
\begin{array}{llll}
(0,0)-0 & (0,1)--1 / 4 & (7,0)--3 / 4 & (7,1)-0 \\
(1,0)--13 / 28 & (1,1)--5 / 7 & (8,0)--5 / 7 & (8,1)--2 / 7 \\
(2,0)--6 / 7 & (2,1)--3 / 28 & (9,0)--17 / 28 & (9,1)--6 / 7 \\
(3,0)--5 / 28 & (3,1)--3 / 7 & (10,0)--3 / 7 & (10,1)--19 / 28 \\
(4,0)--3 / 7 & (4,1)--19 / 28 & (11,0)--5 / 28 & (11,1)--3 / 7 \\
(5,0)--17 / 28 & (5,1)--6 / 7 & (12,0)--6 / 7 & (12,1)--3 / 28 \\
(6,0)--5 / 7 & (6,1)--2 / 7 & (13,0)--13 / 28 & (13,1)--5 / 7
\end{array}
$$

When we test to see which of these are perpendicular to $(7,1)$ (by adding $(7,1)$ and comparing values) we find that we have the following elements (arranged by 
coset of $(7,1))$ and values:

$\begin{array}{lll}(0,0) & (7,1) & 0 \\ (2,0) & (9,1) & -6 / 7 \\ (4,0) & (11,1) & -3 / 7 \\ (6,0) & (13,1) & -5 / 7 \\ (8,0) & (1,1) & -5 / 7 \\ (10,0) & (3,1) & -3 / 7 \\ (12,0) & (5,1) & -6 / 7\end{array}$

Examining the table shows that this form is $w_{7,1}^{1}$, so $\operatorname{Pic}(S)=E_{8} \perp A_{6} \perp U$.

\section{$3.36 \quad(2,3,5,10)$}

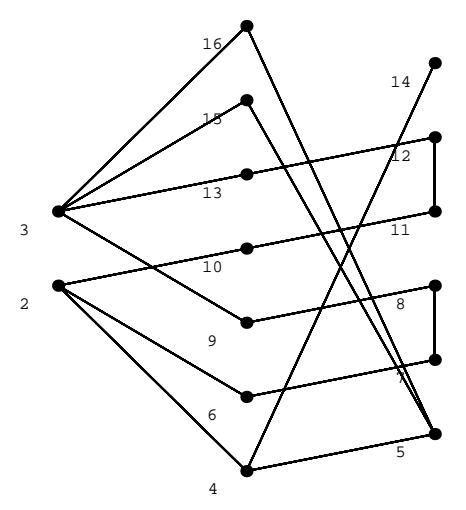

A representative equation for this family of hypersurfaces is $x^{10}+x y^{6}+y^{5} z+z^{4}+w^{2}$.

The Fibration. $\rho=13$ and curve 5 has genus 1. Staring at the graph for hours will show that no fibration exists which has good rank; therefore, we assume that $M W$ is infinite.

Method: Matrix Calculations. Mathematica shows that the discriminant of this form is 20. Among the generators, there are a 5/4-valued generator of order 4 and an 8/5-valued generator of order 5 which are independent. This completely determines the form, which is $w_{2,2}^{5} \perp w_{5,1}^{-1}$. Furthermore, this is of index 1 in $\operatorname{Pic}(S)$ because the 
form has no isotropic subgroups. Therefore, $\operatorname{Pic}(S)=T_{2,5,5} \perp A_{3}$.

\section{$3.37(1,3,4,8)$}

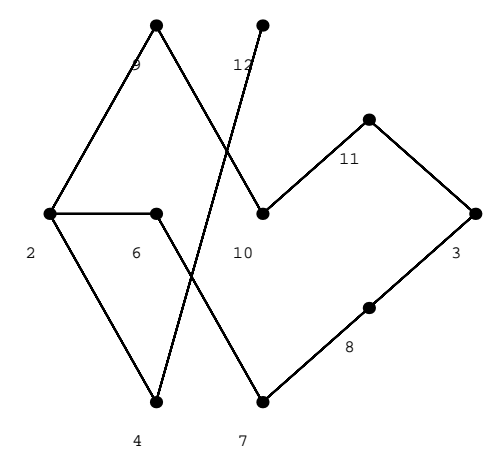

A representative equation for this family of hypersurfaces is $x^{16}+x y^{5}+y^{4} z+z^{4}+w^{2}$. This is one of Arnold's 14 original surface singularities. $\rho=9$. Curve 3 has genus 1 . Note that aside from curve 3 , we have a $T_{3,4,4}$. This lattice has no representation as a sum of Dynkin lattices.

\section{$3.38(1,6,8,15)$}

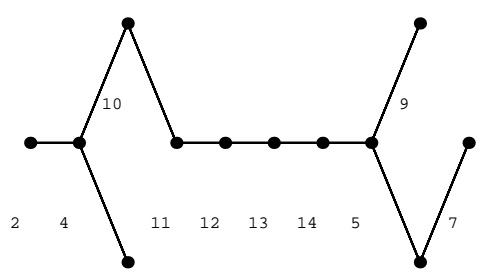

A representative equation for this family of hypersurfaces is $-x^{30}+y^{5}+x^{6} z^{3}+y z^{3}+w^{2}$. This is one of Arnold's 14 original surface singularities. $\rho=11$ and curve 2 has genus 
1 ; if we consider it as a fibre, then curve 4 is a section and the remaining curves form an $\tilde{E}_{8}$ and an $\tilde{A}_{1}$. This fibration has good rank and the associated form has no isotropic subgroups, so $\operatorname{Pic}(S)=E_{8} \perp A_{1} \perp U$.

\section{$3.39 \quad(1,3,5,9)$}

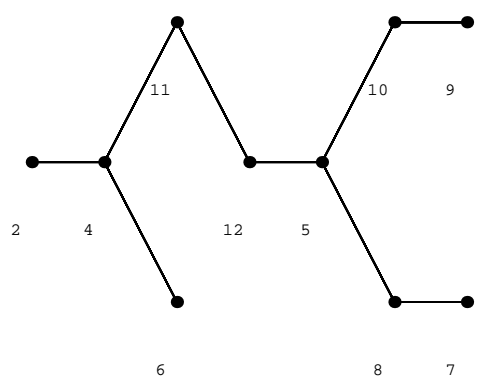

A representative equation for this family of hypersurfaces is $x^{18}+y^{6}+x^{3} z^{3}+y z^{3}+w^{2}$. This is one of Arnold's 14 original surface singularities. $\rho=9$ and curve 2 has genus 1; if we consider it as a fibre, then curve 4 is a section and the remaining curves form an $\tilde{E}_{6}$ and an $\tilde{A}_{1}$. This fibration has good rank and the associated form has no isotropic subgroups, so $\operatorname{Pic}(S)=E_{6} \perp A_{1} \perp U$.

\section{$3.40(1,2,4,7)$}

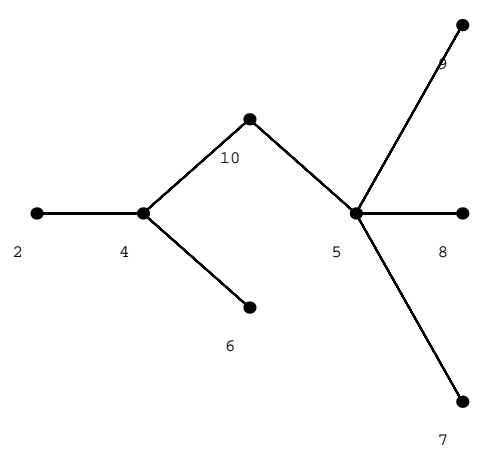


A representative equation for this family of hypersurfaces is $-x^{14}+y^{7}+x^{2} z^{3}+y z^{3}+w^{2}$. $\rho=7$ and curve 2 has genus 1 . If we consider it to be a fibre, then curve 4 is a section and curves 5, 7-10 form a $\tilde{D}_{4}$ while curve 6 can be completed to form an $\tilde{A}_{1}$. This fibration has good rank. There are no isotropic subgroups of the lattice $D_{4} \perp A_{1} \perp U$ so the index in $\operatorname{Pic}(S)$ must be $1 ; \operatorname{Pic}(S)=D_{4} \perp A_{1} \perp U$.

\section{$3.41(2,3,7,12)$}

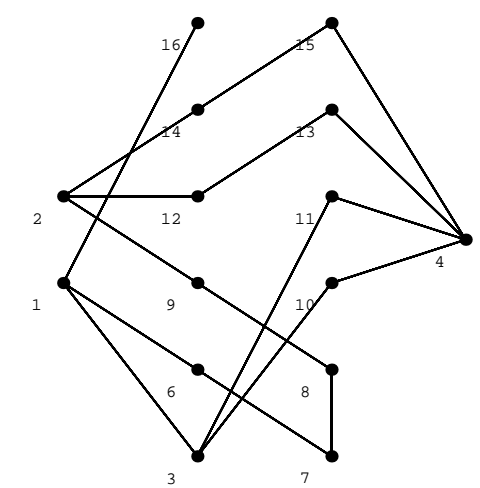

A representative equation for this family of hypersurfaces is $x^{12}+y^{8}+x^{5} z^{2}+y z^{3}+w^{2}$.

The Fibration. $\rho=13$ and curve 4 has genus 1. We may see curves 2, 8, 9, 12-15 as an $\tilde{E}_{6}$ and $1,3,6,10,11,16$ as a $\tilde{D}_{5}$, where curve 7 is then a section and curve 4 is a 4 -section. This has good rank.

The Answer. We know that $M W$ is trivial because $M W$ injects into the discriminant group of each fibre (see [Miranda, p.70]) and the orders of the discriminant groups of $D_{5}$ and $E_{6}$ are coprime. Therefore $\operatorname{Pic}(S)=E_{6} \perp D_{5} \perp U$. 


\section{$3.42(1,1,3,5)$}

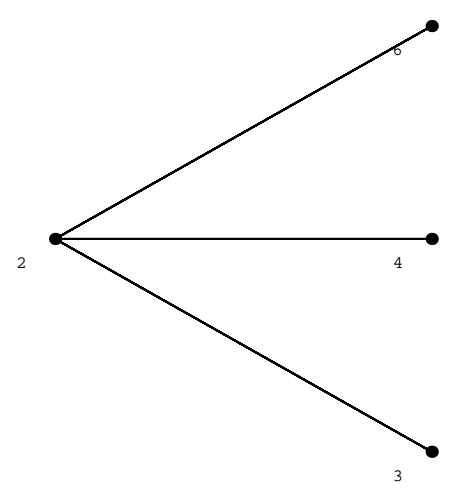

A representative equation for this family of hypersurfaces is $x^{10}+y^{10}+x z^{3}+y z^{3}+w^{2}$. $\rho=3$ and curves 3 and 4 have genus 1 . If we consider them to be fibres, then curve 2 is a section and curve 6 may be completed to an $\tilde{A}_{1}$. This fibration has good rank and the corresponding form has no isotropic subgroups, so $\operatorname{Pic}(S)=A_{1} \perp U$.

\section{$3.43(3,4,11,18)$}

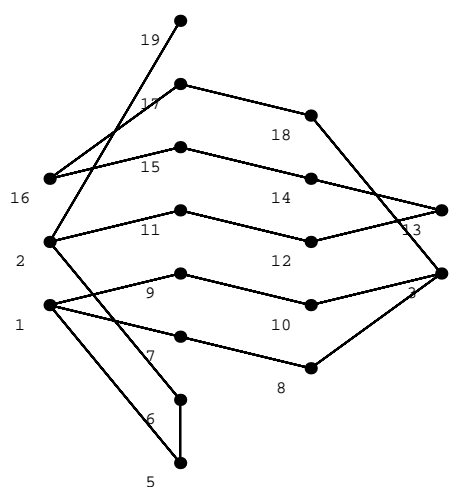

A representative equation for this family of hypersurfaces is $x^{12}+y^{9}+x z^{3}+w^{2}$.

$\rho=16$ and curve 1 has genus 1 . We consider curves 2, 5, 6, 11-15, 19 as forming an $\tilde{E}_{8}$, so that curve 16 is a section, curve 1 is a 2 -section, and curves $3,7-10$, 17, 18 form an $\tilde{E}_{6}$. This fibration has good rank and the index $\operatorname{in} \operatorname{Pic}(S)$ is 1 , so $\operatorname{Pic}(S)=E_{8} \perp E_{6} \perp U$ 


\section{$3.44(1,2,5,8)$}

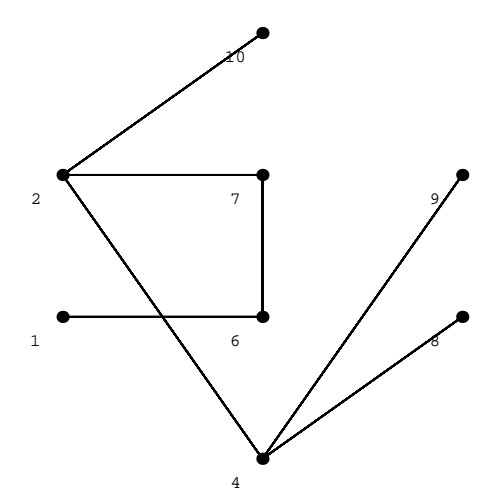

A representative equation for this family of hypersurfaces is $x^{16}+y^{8}+x z^{3}+y^{3} z^{2}+w^{2}$. $\rho=7$ and curve 1 has genus 1 . If we consider it to be a fibre, then curve 6 is a section and we are left with curves 2, 4, 7-10 forming a $\tilde{D}_{5}$. This fibration has good rank and the form associated to $D_{5}$ has no isotropic subgroups, $\operatorname{so} \operatorname{Pic}(S) \cong D_{5} \perp U$.

\section{$3.45(1,4,9,14)$}

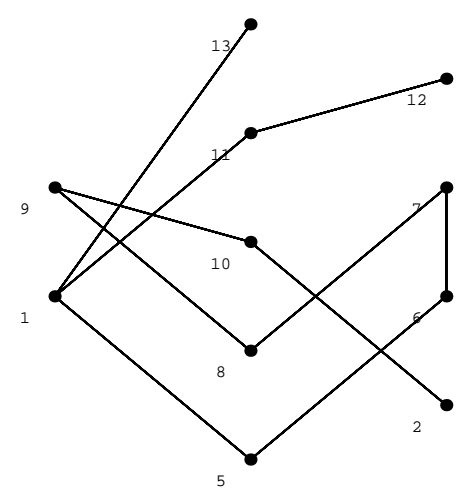

A representative equation for this family of hypersurfaces is $x^{28}+y^{7}+x z^{3}+w^{2}$.

$\rho=10$ and curve 2 has genus 1 . If we consider it to be a fibre, then curve 10 is a section and the remaining curves form an $\tilde{E}_{8}$. This fibration has good rank and because we have an $\tilde{E}_{8}$, the index in $\operatorname{Pic}(S)$ is 1 and so $\operatorname{Pic}(S)=E_{8} \perp U$. 


\section{$3.46(5,6,22,33)$}

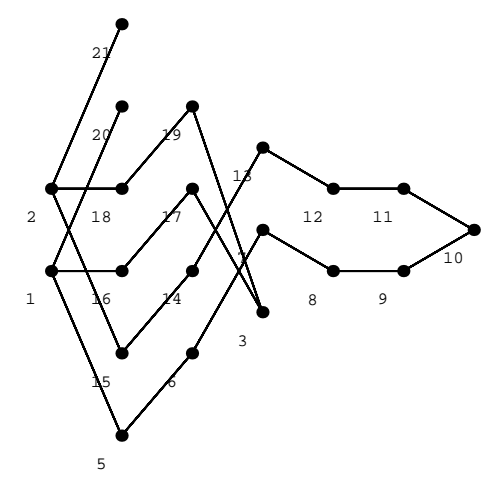

A representative equation for this family of hypersurfaces is $x^{12} y+y^{11}+z^{3}+w^{2}$.

The Fibration. $\rho=18$ and curve 3 has genus 1 . If we consider it as a fibre, then curves 17 and 19 are sections and the remaining curves form a $\tilde{D}_{16}$.

Method: Intermediate Lattice Calculation. The form associated to $D_{16}$ is $u$; we can take either $(0,1)$ or $(1,0)$ to be the generator of an isotropic subgroup; we'll use $(0,1)$ for convenience. In $(0,1)^{\perp}$ we have $(0,0)$ and $(0,1)$ which are in the same coset so we are left with the trivial form. Therefore $\operatorname{Pic}(S)=E_{8} \perp E_{8} \perp U$.

\section{$3.47(3,4,14,21)$}

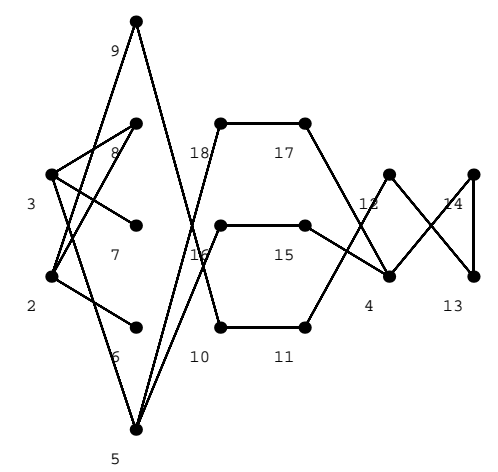

A representative equation for this family of hypersurfaces is $x^{14}+x^{2} y^{9}+y^{7} z+z^{3}+w^{2}$. $\rho=15$ and curve 5 has genus 1 . We consider curve 5 as a 2 -section, then we may see 
curves 4, 13-18 as forming an $\tilde{E}_{6}$, curve 12 as a section, and curves $2,3,6-11$ as an $\tilde{E}_{7}$. This does have good rank, and because $M W$ must embed in the discriminant groups of $E_{6}$ and $E_{7}$ (which have coprime orders), $M W$ must be trivial. Therefore $\operatorname{Pic}(S)=E_{7} \perp E_{6} \perp U$.

\section{$3.48(3,5,16,24)$}

A representative equation for this family of hypersurfaces is $x^{16}+x y^{9}+z^{3}+w^{2}$. See (3.43). They have the same rank and configuration of curves.

\section{$3.49(2,5,14,21)$}

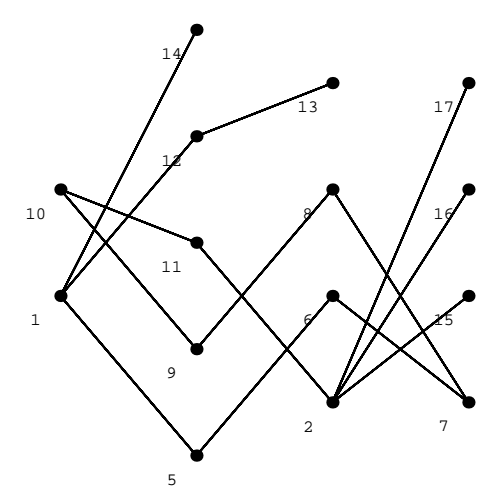

A representative equation for this family of hypersurfaces is $x^{21}+x y^{8}+z^{3}+w^{2}$.

$\rho=14$ and none of the curves have genus 1 . We can, however, see curves 1, 5-9, 12-14 as an $\tilde{E}_{8}$, so that curve 10 is a section and the remaining curves $2,11,15-17$ form a $\tilde{D}_{4}$. Because we have an $E_{8}$ with trivial discriminant group, $M W$ is trivial and $\operatorname{Pic}(S)=E_{8} \perp D_{4} \perp U$. 


\section{$3.50(1,4,10,15)$}

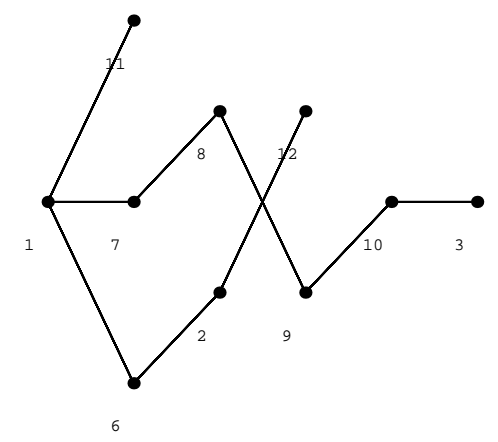

A representative equation for this family of hypersurfaces is $x^{30}+x^{2} y^{7}+y^{5} z+z^{3}+w^{2}$. This is one of Arnold's 14 original surface singularities. $\rho=9$ and curve 3 has genus 1. If we consider curve 3 as a fibre, then curve 10 is a section and we are left with a $\tilde{E}_{7}$. This fibration has good rank and the associated form has no isotropic subgroups, so $\operatorname{Pic}(S)=E_{7} \perp U$.

\section{$3.51(1,5,12,18)$}

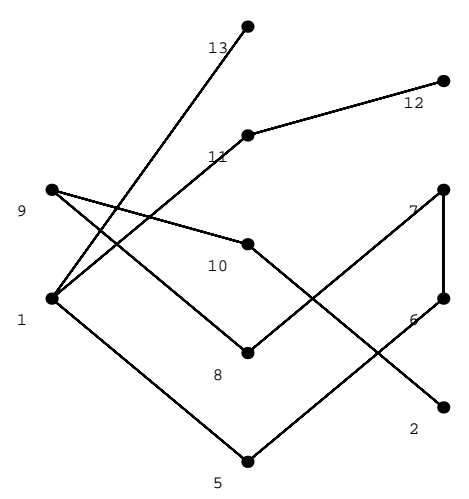

A representative equation for this family of hypersurfaces is $x^{36}+x y^{7}+z^{3}+w^{2}$.

$\rho=10$ and curve 2 has genus 1 . If we consider it as a fibre, then curve 10 is a section and the remaining curves form an $\tilde{E}_{8}$. This fibration has good rank; therefore $\operatorname{Pic}(S) \cong E_{8} \perp U$. 


\section{$3.52(7,8,9,12)$}

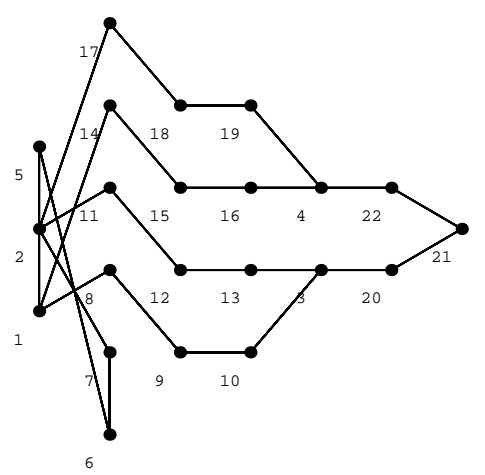

A representative equation for this family of hypersurfaces is $x^{4} y+y^{3} w+z^{4}+w^{3}$.

The Fibration. $\rho=19$; there are no curves of genus 1 . Though one cannot see this from the picture above, this graph has symmetry group $\mathbb{Z}_{4}$ on the curves $1,2,3,4$. We view curves $3,9,10,12,13,20,21$ as an $\tilde{E}_{6}$, curves 8, 11, 22 as sections, and the remaining curves as an $\tilde{A}_{11}$. The $\tilde{A}_{11}$ is somewhat difficult to see in this picture; also, curves 1 and 5 are not connected to curve 2, but only to each other (much as one might think otherwise from the presentation of this graph).

Method: Intermediate Lattice Calculation. The only isotropic subgroup is generated by $(4,1)$. We have 36 elements and corresponding values:

$$
\begin{array}{cll}
(0,0)-0 & (0,1)--2 / 3 & (0,2)--2 / 3 \\
(1,0)--11 / 24 & (1,1)--1 / 8 & (1,2)--1 / 8 \\
(2,0)--5 / 6 & (2,1)--1 / 2 & (2,2)--1 / 2 \\
(3,0)--1 / 8 & (3,1)--19 / 24 & (3,20)--19 / 24 \\
(4,0)--1 / 3 & (4,1)-0 & (4,2)-0 \\
(5,0)--11 / 24 & (5,1)--1 / 8 & (5,2)--1 / 8
\end{array}
$$




$$
\begin{array}{rll}
(6,0)--1 / 2 & (6,1)--1 / 6 & (6,2)--1 / 6 \\
(7,0)--11 / 24 & (7,1)--1 / 8 & (7,2)--1 / 8 \\
(8,0)--1 / 3 & (8,1)-0 & (8,2)-0 \\
(9,0)--1 / 8 & (9,1)--19 / 24 & (9,2)--19 / 24 \\
(10,0)--5 / 6 & (10,1)--1 / 2 & (10,2)--1 / 2 \\
(11,0)--11 / 24 & (11,1)--1 / 8 & (11,2)--1 / 8
\end{array}
$$

When we test to see which of these are perpendicular to $(4,1)$ we find that we have the following elements (arranged by coset of $(4,1)$ ) and values:

$\begin{array}{llll}(0,0) & (4,1) & (8,2) & 0 \\ (3,0) & (7,1) & (11,2) & -1 / 8 \\ (6,0) & (10,1) & (2,2) & -1 / 2 \\ (9,0) & (1,1) & (5,2) & -1 / 8\end{array}$

The element $(3,0)$ is of order 4 so the corresponding form is $w_{2,2}^{-1}$. Therefore $\operatorname{Pic}(S)=E_{8} \perp D_{9} \perp U$.

\section{$3.53(3,4,5,6)$}

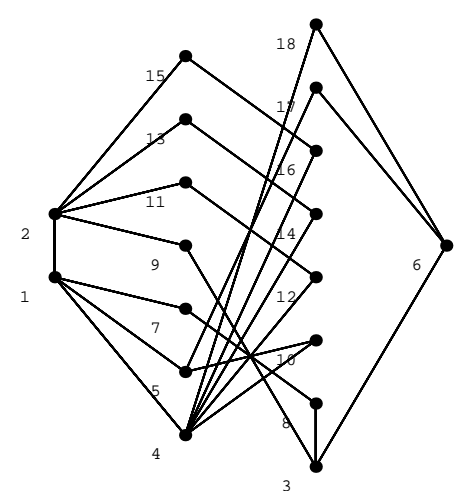

A representative equation for this family of hypersurfaces is $x^{6}+x^{2} y^{3}+y^{3} w+y^{2} z^{2}+$ $x z^{3}+w^{3}$. 
The Fibration. $\rho=15$ and curve 1 has genus 1 . There is no Jacobian fibration which has good rank; we view curves $2,11-16$ as an $\tilde{E}_{6}$, and curves $3,5-8,10,17,18$ as an $\tilde{E}_{7}$, which leaves curves 1, 4, 9 as 3 -sections. The Jacobian fibration associated to this has good rank.

Method: Mukai and Matrix. We can apply Mukai's theorem to see that $\operatorname{Pic}(S)$ is of index 3 in $\operatorname{Pic}(\mathbf{J}(S))$, so that $\operatorname{disc} \operatorname{Pic}(S)=4 \cdot \operatorname{Pic}(\mathbf{J}(S))$ and the intersection matrix for our graph determines $\operatorname{Pic}(S)$. The matrix has discriminant 54 . We have order-9 generators with value $2 / 9$ and $8 / 9$ and order-3 generators with value $2 / 3$, so part of our form is $w_{3,2}^{-1} \perp w_{3,1}^{-1}$. The form for $\operatorname{Pic}(S)$ must embed in $w_{2,1}^{1} \perp w_{3,1}^{-1}$ so we can be sure that the form on $\mathbb{Z}_{2}$ is $w_{2,1}^{1}$. Thus, $\operatorname{Pic}(S)=w_{2,1}^{1} \perp w_{3,2}^{-1} \perp w_{3,1}^{-1}$. This lattice has no representation as a sum of Dynkin lattices. However, we can express this form as $M_{(1,2,2,2,3,4),(1,1,1,1,1,1),-4}$.

\section{$3.54(3,5,6,7)$}

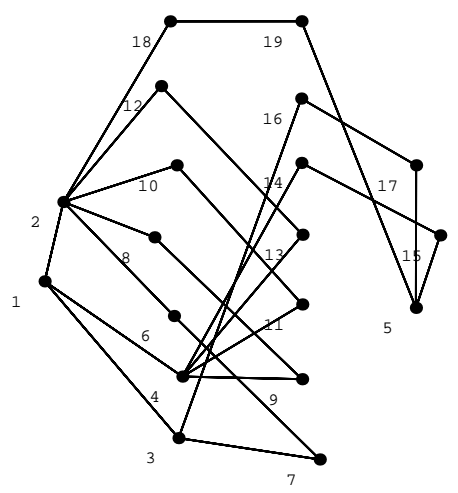

A representative equation for this family of hypersurfaces is $-x^{7}+x^{2} y^{3}+y^{3} z+x z^{3}+$ $w^{3}$.

The Fibration. $\rho=16$ and curve 1 has genus 1 . If we consider it to be a fibre, then curves 2, 3, 4 are sections. Then we may see curves $6-7,8-9,10-11,12-13$ as 
$4 \tilde{A}_{2}$ and curves 5, 14-19 as an $\tilde{E}_{6}$. This fibration has good rank.

The Answer. This fibration is the same as that of number 16 (see 3.16), so we have the same lattice: $\operatorname{Pic}(S)=E_{8} \perp\left(A_{2}\right)^{3} \perp U$.

\section{$3.55 \quad(2,5,6,7)$}

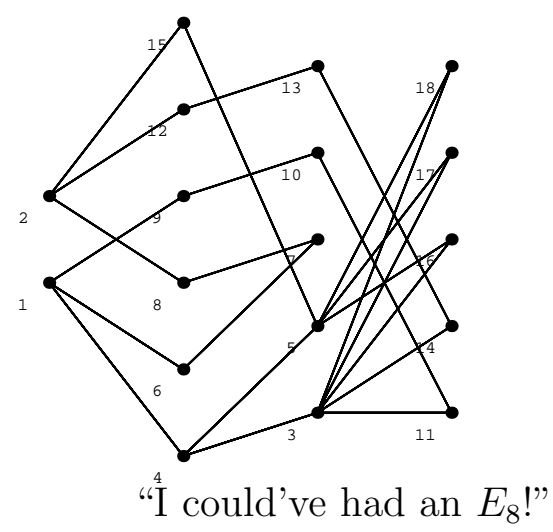

A representative equation for this family of hypersurfaces is $-x^{10}+y^{4}+x z^{3}+x^{3} w^{2}+$ $z w^{2}$.

The Fibration. $\rho=15$ and curves 4 and 5 have genus 1 . The edge between curves 4 and 5 has multiplicity 2. If we consider curve 4 as a fibre, then curves 1 and 3 are sections and we may see curves $2,6-8,12-15$ as an $\tilde{E}_{7}$, and complete curves 16-18 to $3 \tilde{A}_{1}$ and curves 9-11 to an $\tilde{A}_{3}$. This fibration has good rank.

Method: Intermediate Lattice Calculation. The value for $E_{7}$ is $1 / 2$, for $A_{1}$ is $-1 / 2$, and for $A_{3}$ is $5 / 4$. Thus the only isotropic subgroups, up to permutation of the entries, are generated by $(1,1,0,0,0),(0,0,1,1,2)$, and $(1,1,1,1,2)$.

We will begin with $(1,1,0,0,0)$ and leave off the last three entries. Here is a table of elements by coset, their values, and whether they are in $\langle(1,1)\rangle^{\perp}$ or not. 


$$
\begin{array}{lllll}
(0,0) & 0 & (1,1) & 0 & \text { yes } \\
(0,1) & -1 / 2 & (1,0) & 1 / 2 & \text { no }
\end{array}
$$

Therefore we have only the trivial form and are left with $\left(w_{2,1}^{-1}\right)^{2} \perp w_{2,2}^{5}$.

Now for $(0,0,1,1,2)$. We will leave off the first two entries. Here is a table of elements by coset and their values.

$$
\begin{array}{llllllll}
(0,0,0) & 0 & (1,1,2) & 0 & (0,1,0) & -1 / 2 & (1,0,2) & 1 / 2 \\
(0,0,1) & 5 / 4 & (1,1,3) & 1 / 4 & (0,1,1) & 3 / 4 & (1,0,3) & 3 / 4 \\
(0,0,2) & 1 & (1,1,0) & -1 & (0,1,2) & 1 / 2 & (1,0,0) & -1 / 2 \\
(0,0,3) & 5 / 4 & (1,1,1) & 1 / 4 & (0,1,3) & 3 / 4 & (1,0,1) & 3 / 4
\end{array}
$$

So those in $\langle(1,1,2)\rangle^{\perp}$ are

$$
\begin{array}{lll}
(0,0,0) & (1,1,2) & 0 \\
(0,0,2) & (1,1,0) & 1 \\
(0,1,1) & (1,0,3) & 3 / 4 \\
(0,1,3) & (1,0,1) & 3 / 4
\end{array}
$$

The corresponding form is $w_{2,2}^{-5}$, so we have $w_{2,1}^{1} \perp w_{2,1}^{-1} \perp w_{2,2}^{-5}$.

Finally, we do $(1,1,1,1,2)$. Here is a table of elements by coset (up to permutation of the entries), their values, and whether they are in $\langle(1,1,1,1,2)\rangle^{\perp}$ or not.

$$
\begin{array}{llllllllll}
(0,0,0,0,0) & 0 & (1,1,1,1,2) & 0 & \text { yes } & (0,0,0,1,0) & -1 / 2 & (1,1,1,0,2) & 1 / 2 & \text { no } \\
(0,0,0,0,1) & 5 / 4 & (1,1,1,1,3) & 1 / 4 & \text { no } & (0,0,0,1,1) & 3 / 4 & (1,1,1,0,3) & 3 / 4 & \text { yes } \\
(0,0,0,0,2) & 1 & (1,1,1,1,0) & -1 & \text { yes } & (0,0,0,1,2) & 1 / 2 & (1,1,1,0,0) & -1 / 2 & \text { no } \\
(0,0,0,0,3) & 5 / 4 & (1,1,1,1,1) & 1 / 4 & \text { no } & (0,0,0,1,3) & 3 / 4 & (1,1,1,0,1) & 3 / 4 & \text { yes }
\end{array}
$$




$\begin{array}{llllllllll}(0,0,1,1,0) & -1 & (1,1,0,0,2) & 1 & \text { yes } & (0,1,1,1,0) & -3 / 2 & (1,0,0,0,2) & 3 / 2 & \text { no } \\ (0,0,1,1,1) & 1 / 4 & (1,1,0,0,3) & 5 / 4 & \text { no } & (0,1,1,1,1) & -1 / 4 & (1,0,0,0,3) & 7 / 4 & \text { yes } \\ (0,0,1,1,2) & 0 & (1,1,0,0,0) & 0 & \text { yes } & (0,1,1,1,2) & -1 / 2 & (1,0,0,0,0) & 1 / 2 & \text { no } \\ (0,0,1,1,3) & 1 / 4 & (1,1,0,0,1) & 5 / 4 & \text { no } & (0,1,1,1,3) & -1 / 4 & (1,0,0,0,1) & 7 / 4 & \text { yes }\end{array}$

This leaves us with (arranged by coset):

$$
\begin{array}{llllll}
(0,0,0,0,0) & (1,1,1,1,2) & 0 & (0,0,1,1,0) & (1,1,0,0,2) & 1 \\
(0,0,0,0,2) & (1,1,1,1,0) & 1 & (0,1,0,1,0) & (1,0,1,0,2) & 1 \\
(0,0,0,1,1) & (1,1,1,0,3) & 3 / 4 & (0,1,1,0,0) & (1,0,0,1,2) & 1 \\
(0,0,1,0,1) & (1,1,0,1,3) & 3 / 4 & (0,0,1,1,2) & (1,1,0,0,0) & 0 \\
(0,1,0,0,1) & (1,0,1,1,3) & 3 / 4 & (0,1,0,1,2) & (1,0,1,0,0) & 0 \\
(0,0,0,1,3) & (1,1,1,0,1) & 3 / 4 & (0,1,1,0,2) & (1,0,0,1,0) & 0 \\
(0,0,1,0,3) & (1,1,0,1,1) & 3 / 4 & (0,1,1,1,1) & (1,0,0,0,3) & 7 / 4 \\
(0,1,0,0,3) & (1,0,1,1,1) & 3 / 4 & (0,1,1,1,3) & (1,0,0,0,1) & 7 / 4
\end{array}
$$

All of the 1-valued elements and 0-valued elements (aside from the identity) are of order 2 ; the rest are of order 4 . So we have the group $\mathbb{Z}_{2} \oplus \mathbb{Z}_{2} \oplus \mathbb{Z}_{4}$. The form on $\mathbb{Z}_{4}$ must be $w_{2,2}^{-1}$ as there is one copy of the values for this form; because the other values are 0,1 the other elements must correspond to the form $u$ or $v$. Testing each shows that our form must be $w_{2,2}^{-1} \perp v$.

We have different answers, and now we need to determine which of the isotropic subgroups is the correct one. The only way to do this is to (a) exhibit a fibration corresponding to one of the forms and (b) show that none of the other lattices can be exhibited as a fibration. There are three possibilities for a fibration here: $E_{8} \perp A_{3} \perp\left(A_{1}\right)^{2} \perp U, E_{7} \perp D_{5} \perp A_{1} \perp U$, and $D_{4} \perp D_{9} \perp U$. With much pain, one may show that the first two of these are not possible, no matter what kind of curves are added. 
We may see the last one in the following way. We can see curves $3,14,16-18$ as forming a $\tilde{D}_{4}$, so that curve 13 is a section and curve 11 is a 2 -section and curve 5 is a 3 -section. Then curves $1,2,6-10,12,15$ may be completed to form a $\tilde{D}_{9}$ by adding a curve which intersects curve 9 .

Thus, $\operatorname{Pic}(S)=D_{9} \perp D_{4} \perp U$.

\section{$3.56 \quad(5,6,8,11)$}

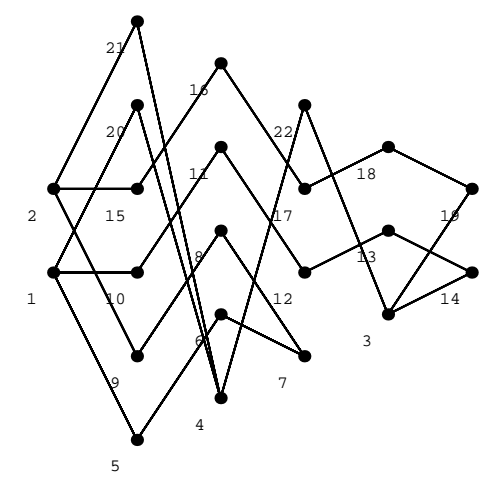

A representative equation for this family of hypersurfaces is $x^{6}+y^{5}+y z^{3}+z w^{2}$.

The Fibration. Curve 4 has genus 1. If we consider it as a fibre, then curves 20, 21, and 22 are sections. The remaining curves form an $\tilde{A}_{17}$. As $\rho=19$, this fibration has good rank.

Method: Intermediate Lattice Calculation. The discriminant group for $\tilde{A}_{17}$ is $\mathbb{Z}_{18}$, with generator value $-17 / 36(\bmod \mathbb{Z})$. It has a $q$-isotropic subgroup generated by $(6)$.

Here are the elements and their values: 

(0) 0
(5) $-29 / 36$
(1) $-17 / 36$
(6) $\quad 0$
(2) $-8 / 9$
(3) $-1 / 4$
(4) $-5 / 9$
(7) $-5 / 36$
(8) $-2 / 9$
(9) $-1 / 4$

When we test to see which of these are perpendicular to (6), we find that we have the following elements (arranged by cosets) and values:
(0) (6) (12) $\quad 0$
(3) (9) (15) $-1 / 4$

This corresponds to the form $w_{2,1}^{-1}$ and so we have $\operatorname{Pic}(S)=E_{8} \perp E_{8} \perp A_{1} \perp U$.

\section{$3.57(4,5,6,9)$}

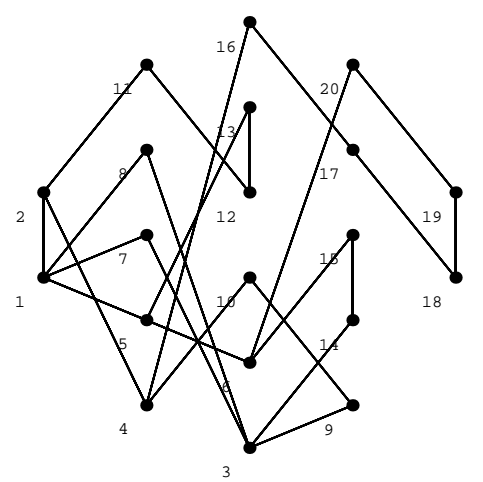

A representative equation for this family of hypersurfaces is $x^{6}+x y^{4}+y^{3} w+z^{4}+z w^{2}$. The Fibration. Curve 1 has genus 1. We may consider curves 2, 4, 9, 10, 16 - 20 as forming an $\tilde{E}_{8}$. Then curves 1 and 11 are 3 -sections, curve 3 is a 2 -section, and curve 6 is a section. We must find a completion for this fibration. If we check the matrix, we find that it has discriminant 12, so whatever fibration we find must have discriminant 3 or 12 in order to comply with Lemma 1.7.3.1. Thus, we must find 
either an $\tilde{E}_{6}$ or an $\tilde{A}_{2}$. There are no discriminant 3 or 12 fibrations of rank 17 with an $\tilde{E}_{6}$ and no discriminant 3 fibration of rank 17 with an $\tilde{A}_{2}$. There is exactly one discriminant 12 fibration of rank 17 with an $\tilde{A}_{2}$, which is $\tilde{E}_{8}+\tilde{A}_{2}+\tilde{D}_{5}$. In fact, we can exhibit this. We complete curves 14,15 to form an $\tilde{A}_{2}$ (and intersect the extra curve with curve 3 ), and add a curve intersecting curves $7,8,13$ to form a $\tilde{D}_{5}$; this curve must also intersect curve 11 twice.

The Answer. As this fibration contains an $\tilde{E}_{8}$, the index of this lattice in $\operatorname{Pic}(S)$ must be 1 and so $\operatorname{Pic}(S)=E_{8} \perp D_{5} \perp A_{2} \perp U$.

\section{$3.58(1,4,5,6)$}

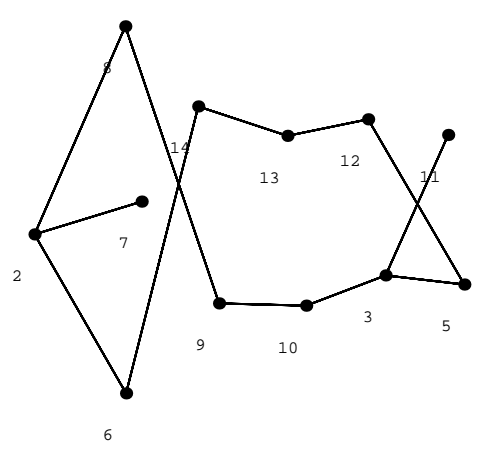

A representative equation for this family of hypersurfaces is $x^{16}+y^{4}+x z^{3}+z^{2} w+$ $x^{4} w^{2}+y w^{2}$.

This is one of Arnold's 14 original surface singularities. $\rho=11$ and curve 5 has genus 1. Note that aside from curve 5 , we have a $T_{2,5,6}$. This lattice has no representation as a sum of Dynkin lattices. 


\section{$3.59(1,5,7,8)$}

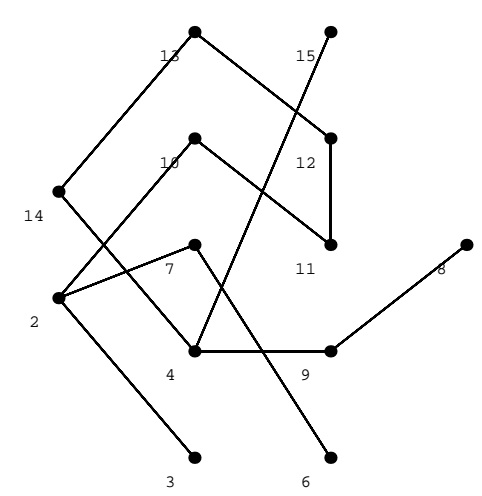

A representative equation for this family of hypersurfaces is $-x^{21}+x y^{4}+z^{3}+x^{5} w^{2}+$ $y w^{2}$.

Curve 3 has genus 1 . If we consider it as a fibre, then curve 2 must be a section. Then curves 4, 8 - 15 easily form an $\tilde{E}_{8}$ and we may complete curves 6 and 7 to an $\tilde{A}_{2}$. As $\rho=12$, we see that this fibration has good rank. Because we have exhibited an $\tilde{E}_{8}$, we know that $|M W|=1$ and $\operatorname{Pic}(S)=E_{8} \perp A_{2} \perp U$.

\section{$3.60(1,4,6,7)$}

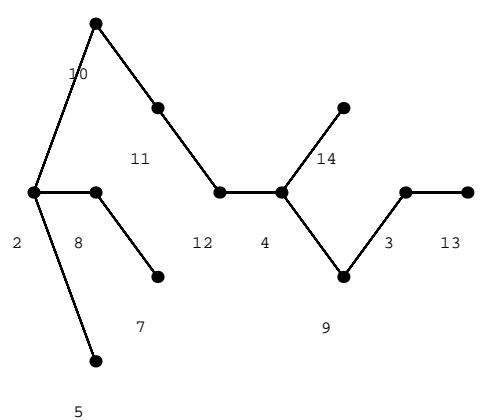

A representative equation for this family of hypersurfaces is $x^{18}+x^{2} y^{4}+y^{3} z+z^{3}+$ $x^{4} w^{2}+y w^{2}$.

This is one of Arnold's 14 original surface singularities. $\rho=11$ and curve 5 has genus 
1. If we consider curve 5 to be a fibre, then curve 2 is a section and we are left with $\tilde{E}_{7}$ and $\tilde{A}_{2}$. This fibration has good rank and the associated form has no isotropic subgroups, so $\operatorname{Pic}(S)=E_{7} \perp A_{2} \perp U$.

\section{$3.61(4,6,7,11)$}

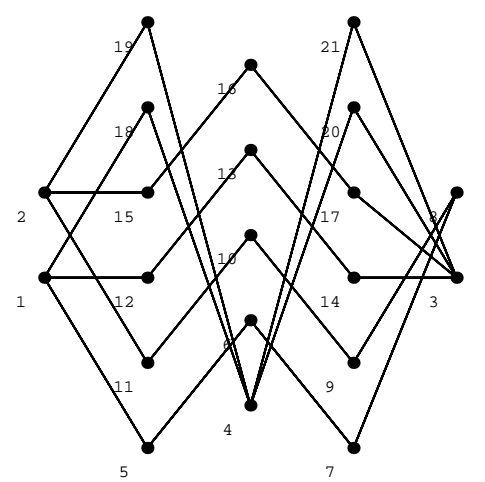

A representative equation for this family of hypersurfaces is $x^{7}+x y^{4}+z^{4}+y w^{2}$.

Curve 4 has genus 1 . We note that curves $2,7-11,15,16$, and 19 form an $\tilde{E}_{8}$, so that curve 6 is a section, curve 17 is a 2 -section, curve 4 is a 3 -section, and the remaining curves form a $\tilde{D}_{8}$. This fibration has good rank. As we have exhibited an $\tilde{E}_{8},|M W|=1$ and so $\operatorname{Pic}(S)=E_{8} \perp D_{8} \perp U$.

\section{$3.62(3,4,5,8)$}

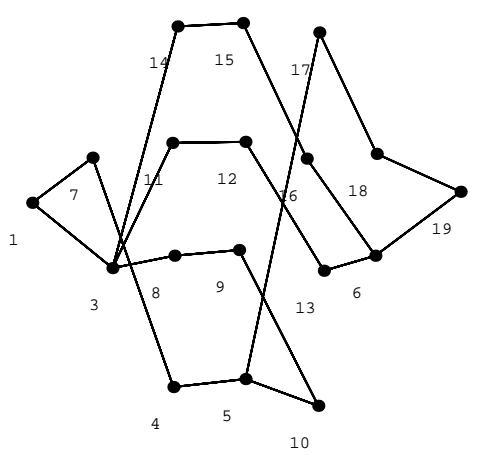


A representative equation for this family of hypersurfaces is $x^{4} y^{2}+y^{5}+x^{5} z+z^{4}+$ $x^{4} w+y w^{2}$.

The Fibrations. There are no genus 1 curves. We have a choice of fibrations: We may see curves $1,4,5,7,10,17-19$ as an $\tilde{E}_{7}$ so that curves 3,6 are sections and curves 11 -13 may be completed to an $\tilde{A}_{3}$, curves 14 - 16 may be completed to an $\tilde{A}_{3}$, curve 8 may be completed to an $\tilde{A}_{1}$, and curve 9 is a 2 -section. As $\rho=16$, this fibration has good rank. However, this leads to a long and painful intermediate lattice calculation, so instead we will look at a better fibration. We can see curves 1, 4, 5, $7-10,17$ as an $\tilde{E}_{7}$, and curves $6,11-16,19$ as another $\tilde{E}_{7}$, so that curves 3 and 18 are 2 -sections. The Jacobian fibration has good rank.

The Method: Mukai and Matrix. Mukai tells us that we may just get the form from the matrix. It has discriminant 16. There are several order-4 generators of value $3 / 4$ and of value $1 / 2$. The sum of two $3 / 4$-value generators has value $1 / 2$. The only way this can come about is if one of the generators has value $3 / 4=7 / 4+1$, where the $7 / 4$ is the value of an order- 4 generator and the 1 is the value of an order 2 generator (twice the 3/4-valued order 4 generator). Thus the two generators for this form have values $3 / 4$ and $7 / 4$.

The Answer. This form, $w_{2,2}^{-1} \perp w_{2,2}^{-5}$, corresponds to the lattice $D_{9} \perp E_{5} \perp U$, so $\operatorname{Pic}(S)=D_{9} \perp E_{5} \perp U$ 


\section{$3.63(1,2,3,4)$}

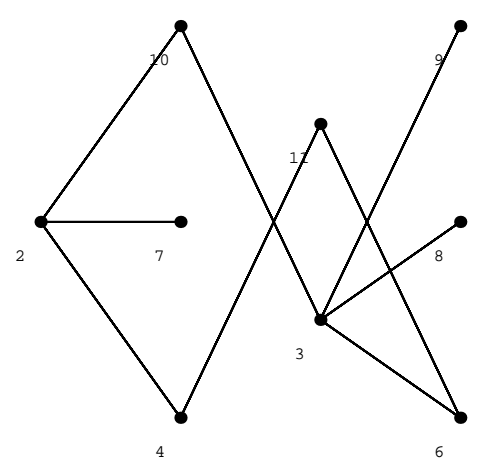

A representative equation for this family of hypersurfaces is $x^{10}+y^{5}+x z^{3}+y^{2} z^{2}+$ $z^{2} w+x^{2} w^{2}+y w^{2}$

The Fibration. Curve 4 has genus 1. Inspection of the graph will convince the reader that there is no possible fibration which indicates that $r k(M W)=0$.

The Method: Examine the Matrix. We learn from Mathematica that the matrix has discriminant 20 . Here are the generator orders and values:

$\begin{array}{lllllllll}\text { order } & 5 & 1 & 10 & 10 & 10 & 2 & 10 & 5 \\ \text { value } & 8 / 5 & 0 & 1 / 10 & 9 / 10 & 7 / 5 & 1 & 9 / 10 & 8 / 5\end{array}$

The value of $8 / 5$ on an order 5 generator tells us that the form must contain $w_{5,1}^{-1}$. We can then see that the remainder of the form is $\left(w_{2,1}^{1}\right)^{2}$ by noting that $9 / 10=2 / 5+1 / 2$ (note that we cannot write it as a sum with $3 / 2$ or we will be using the wrong form on $\mathbb{Z}_{5}$ ). We then see that the value of the sum of the fourth and seventh generators is 1, which indicates that the two sub-generators of value $1 / 2$ are independent of each other. Thus, $\operatorname{Pic}(S)=w_{5,1}^{-1} \perp\left(w_{2,1}^{1}\right)^{2}$; this lattice has no isotropic subgroups and cannot be expressed as a sum of Dynkin lattices. However, we can express this form as $M_{(1,1,2,3),(1,1,1,1),-2}$. 


\section{$3.64 \quad(3,4,7,10)$}

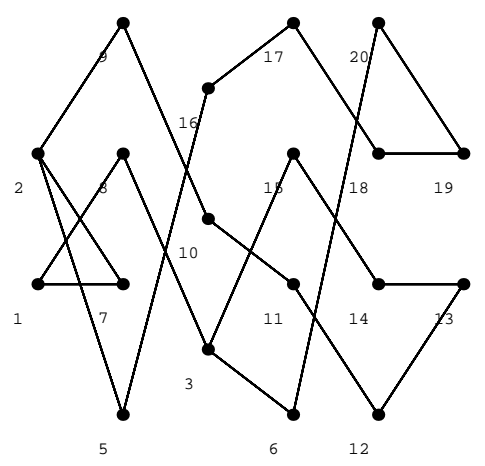

A representative equation for this family of hypersurfaces is $x^{8}+y^{6}+x z^{3}+z^{2} w+y w^{2}$.

The Fibration. Curve 1 has genus 1 and if we consider it to be a fibre, then curves 7 and 8 are sections. The remaining curves form an $\tilde{A}_{15}$. This fibration has good rank as $\rho=17$.

Method: Intermediate Lattice Calculation. The discriminant group for $\tilde{A}_{15}$ is $\mathbb{Z}_{16}$ and the value of the generator of the discriminant form is $-15 / 32(\bmod \mathbb{Z})$. There is one isotropic subgroup, generated by (8). The even elements are perpendicular to (8), and after modding out by (8), we have:

$$
0-0 \quad 2--7 / 8 \quad 4--1 / 2 \quad 6--7 / 8
$$

This form is $w_{2,2}^{1}$. Thus, $\operatorname{Pic}(S)=E_{8} \perp E_{7} \perp U$.

\section{$3.65(3,5,11,14)$}

A representative equation for this family of hypersurfaces is $x^{11}+x y^{6}+z^{3}+y w^{2}$ See (3.46). They have the same rank and configuration of curves. 


\section{$3.66(1,1,2,3)$}

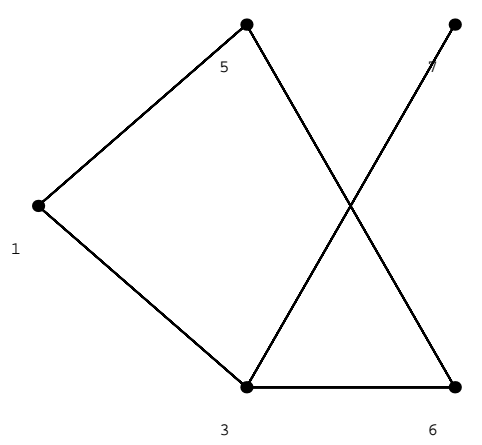

A representative equation for this family of hypersurfaces is $x^{7}+y^{7}+x z^{3}+y z^{3}+$ $z^{2} w+x w^{2}+y w^{2}$

The Fibration. Curves 1 and 6 have genus 1; if we consider these as fibres, then curves 3 and 5 are sections and we must complete curve 7 to an $\tilde{A}_{1}$. As $\rho=4$, this fibration satisfies Shioda-Tate with $r k(M W)=1$. There is no other possible fibration.

Method: Use the Matrix. When we examine the matrix, we see that it has discriminant 7 and form $w_{7,1}^{1}$. This lattice has no representation as a sum of Dynkin lattices. However, we can express this form as $M_{(1,2),(1,1), 0}$. 


\section{$3.67 \quad(2,3,7,9)$}

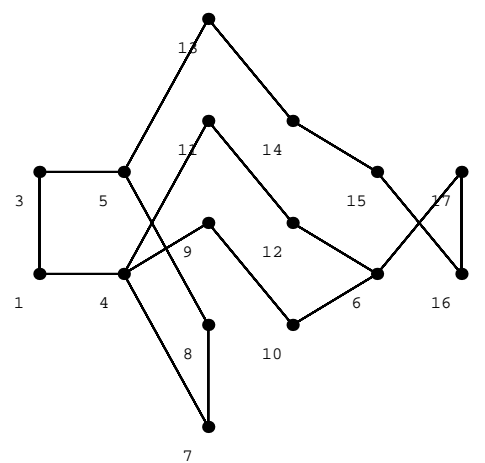

A representative equation for this family of hypersurfaces is $x^{9} y+y^{7}+x^{7} z+z^{3}+$ $x^{6} w+y w^{2}$.

Curve 4 has genus 1 . We see curves $1,3,5,8,13-17$ as forming an $\tilde{E}_{8}$, so that curve 6 is a section, curve 4 is a 2 -section, and curve 7 is a 3 -section. Then we may complete curves 11,12 to an $\tilde{A}_{2}$ and curves 9,10 to an $\tilde{A}_{2}$. This fibration has good rank. Because this fibration contains an $\tilde{E}_{8}$, the index of this lattice in $\operatorname{Pic}(S)$ is 1 so that $\operatorname{Pic}(S)=E_{8} \perp\left(A_{2}\right)^{2} \perp U$.

\section{$3.68(3,4,10,13)$}

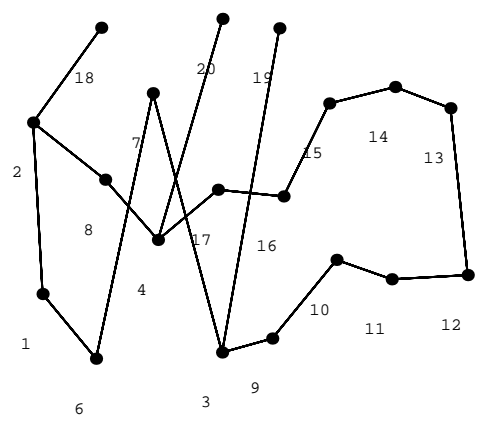

A representative equation for this family of hypersurfaces is $x^{10}+x^{2} y^{6}+y^{5} z+z^{3}+y w^{2}$.

Curve 1 has genus 1 . We notice that curves $3,6,7,9-13$, and 19 form an $\tilde{E}_{8}$, so 
that curve 14 is a section and curve 1 is a 2 -section. This leaves curves $2,4,8,15$ 18, and 20 forming an $\tilde{E}_{7}$. As $\rho=17$, this fibration has good rank. It contains an $\tilde{E}_{8}$, so the index of this lattice in $\operatorname{Pic}(S)$ is 1 , so $\operatorname{Pic}(S)=E_{8} \perp E_{7} \perp U$.

\section{$3.69(2,3,4,7)$}

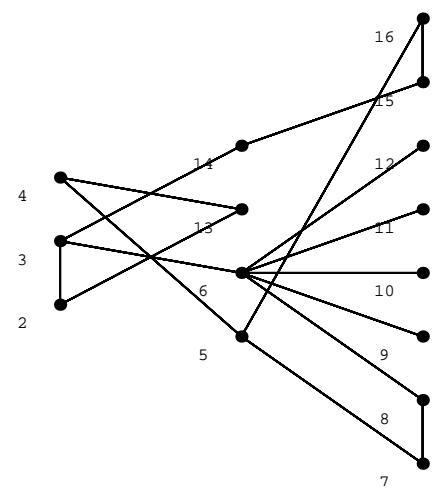

A representative equation for this family of hypersurfaces is $x^{8}+x^{2} y^{4}+y^{4} z+z^{4}+$ $y^{3} w+x w^{2}$.

The Fibration. Curve 3 has genus 1. We may view curves 6, 9 - 12 as forming a $\tilde{D}_{4}$. Then, curves 3 and 8 are 2-sections and curves 2, 4, 5, 7, 13 - 16 form an $\tilde{E}_{7}$. The Jacobian fibration has good rank.

Method: Mukai and the Matrix. We know that the Mukai technique works in this case, so that the form is that which corresponds to the matrix. The matrix has discriminant 32 . Here are the orders and values of the generators of the form:

$\begin{array}{cccccccccccccc}\text { order } & 1 & 1 & 1 & 4 & 4 & 2 & 8 & 8 & 8 & 8 & 4 & 2 & 4 \\ \text { value } & 0 & 0 & 0 & 1 / 2 & 1 / 2 & 0 & 9 / 8 & 9 / 8 & 9 / 8 & 9 / 8 & 1 / 2 & 0 & 1 / 2\end{array}$

Because this lattice must embed in the lattice $w_{2,1}^{1} \perp v$, the only possibilities for this lattice are $w_{2,3}^{1} \perp v$ and $w_{2,3}^{5} \perp v$. (See 3.19 for explanation.) Because the value on 
the sum of generators 7 and 8 is $3 / 2$, we deduce that we have the form $w_{2,3}^{1} \perp v$. Thus, $\operatorname{Pic}(S)=D_{4} \perp A_{7} \perp U$.

\section{$3.70(2,3,5,8)$}

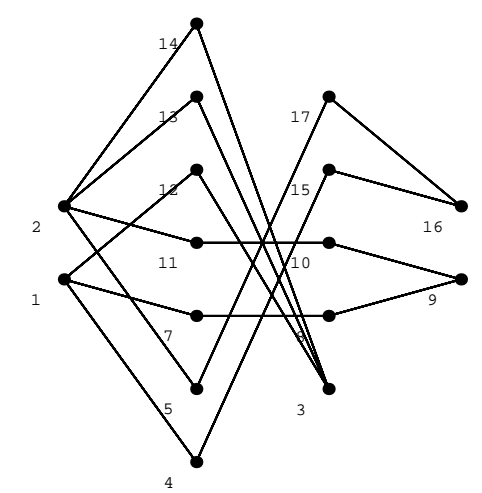

A representative equation for this family of hypersurfaces is $x^{9}+y^{6}+x^{4} z^{2}+y z^{3}+$ $z^{2} w+x w^{2}$

The Fibration. Curve 3 has genus 1. We can see curves 1, 2, 4, 5, 7, 12 - 17 as forming a $\tilde{D}_{10}$; then curve 3 is a 3 -section, curve 8 is a section, and curve 11 is a 2-section. We can then complete curves 9 and 10 to form an $\tilde{A}_{2}$ as long as the extra curve intersects curves 3 and 11 correctly. As $\rho=14$, this fibration has good rank. The Answer. Because $M W$ must embed in the discriminant group of each fibre, and because the orders of these discriminant groups are coprime (4 and 3 respectively), $M W$ must be trivial. Thus, the index of this lattice in the Picard Lattice is 1, and $\operatorname{Pic}(S)=D_{10} \perp A_{2} \perp U$. 


\section{$3.71(1,3,4,7)$}

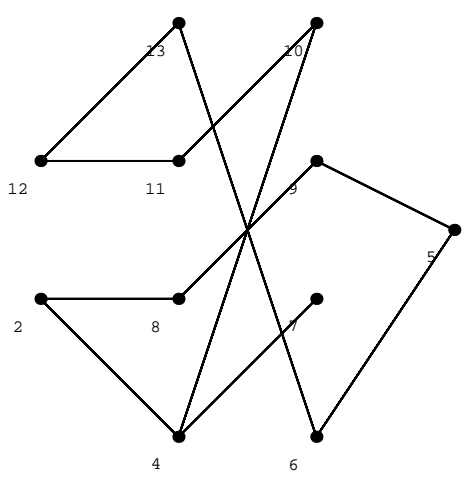

A representative equation for this family of hypersurfaces is $x^{15}+y^{5}+x^{3} z^{3}+y z^{3}+$ $x^{2} w+x w^{2}$.

Curve 6 has genus 1 . We see all curves except curve 6 as forming a $T_{2,5,5}$. This has rank 10, so we need to determine its index in $\operatorname{Pic}(S)$. Mathematica shows that the discriminant of the matrix is 5 , so $\operatorname{Pic}(S)=T_{2,5,5}$.

\section{$3.72(1,2,5,7)$}

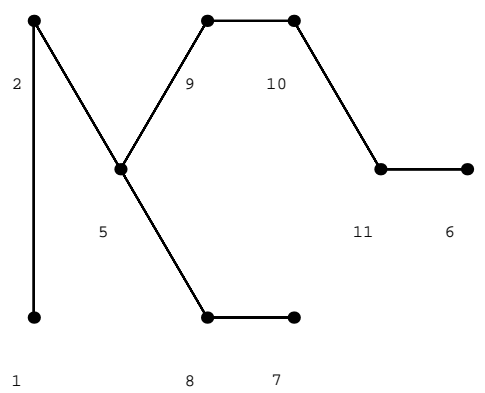

A representative equation for this family of hypersurfaces is $x^{15}+x y^{9}+y^{5} z+z^{3}+$ $y^{4} w+x w^{2}$.

Curve 1 has genus 1 . If we consider it as a fibre, then curve 11 must be a section and the remaining curves form an $\tilde{E}_{6}$. As $\rho=8$, this fibration has good rank. There 
are no isotropic subgroups of $\mathbb{Z}_{3}$ and we have only exhibited one section; we know there can be no more than three sections, and formula Nameless (1.7.3.1) indicates that there can only be one section. Thus, $\operatorname{Pic}(S)=E_{6} \perp U$.

\section{$3.73(7,8,10,25)$}

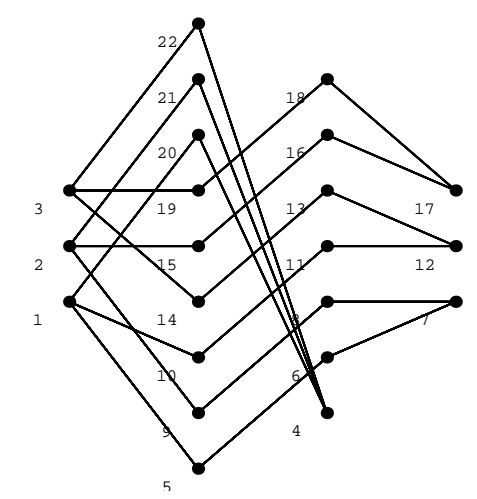

A representative equation for this family of hypersurfaces is $x^{6} y+y^{5} z+z^{5}+w^{2}$.

See section 3.56. Number 56 has the same rank and configuration of curves. (However, this presentation of the graph is more attractive.)

\section{$3.74(4,5,7,16)$}

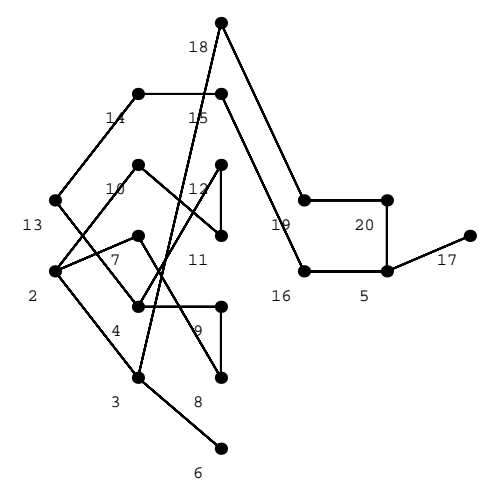

A representative equation for this family of hypersurfaces is $x^{8}+x^{3} y^{4}+y^{5} z+x z^{4}+w^{2}$.

The Fibration. There are no curves of genus 1. We can see curves 5, $14-20$ as 
forming an $\tilde{E}_{7}$, so that curves 3 and 13 are sections, curves $2,4,7$ - 12 form an $\tilde{A}_{7}$, and we can complete curve 6 to form an $\tilde{A}_{1}$. As $\rho=17$, this fibration has good rank. Method: Intermediate Lattice Calculation. The form is $w_{2,1}^{1} \perp w_{2,1}^{-1} \perp w_{2,3}^{1}$. Here are the elements and their values:

$$
\begin{array}{llllllll}
(0,0,0) & 0 & (0,1,0) & 3 / 2 & (1,0,0) & 1 / 2 & (1,1,0) & 0 \\
(0,0,1) & 1 / 8 & (0,1,1) & 13 / 8 & (1,0,1) & 5 / 8 & (1,1,1) & 1 / 8 \\
(0,0,2) & 1 / 2 & (0,1,2) & 0 & (1,0,2) & 1 & (1,1,2) & 1 / 2 \\
(0,0,3) & 9 / 8 & (0,1,3) & 5 / 8 & (1,0,3) & 13 / 8 & (1,1,3) & 9 / 8 \\
(0,0,4) & 0 & (0,1,4) & 3 / 2 & (1,0,4) & 1 / 2 & (1,1,4) & 0 \\
(0,0,5) & 9 / 8 & (0,1,5) & 5 / 8 & (1,0,5) & 13 / 8 & (1,1,5) & 9 / 8 \\
(0,0,6) & 1 / 2 & (0,1,6) & 0 & (1,0,6) & 1 & (1,1,6) & 1 / 2 \\
(0,0,7) & 1 / 8 & (0,1,7) & 13 / 8 & (1,0,7) & 5 / 8 & (1,1,7) & 1 / 8
\end{array}
$$

The three possible isotropic subgroups are $\langle(1,1,0)\rangle,\langle(0,0,4)\rangle$, and $\langle(1,1,4)\rangle$. Here we show the elements which are perpendicular to each subgroup, arranged by coset, and then solve for the associated form.

\begin{tabular}{cccccc}
\multicolumn{7}{c}{$\langle(1,1,0)\rangle$} & & & & \\
$(0,0,0)$ & 0 & $(1,1,0)$ & $(0,0,4)$ & 0 & $(1,1,4)$ \\
$(0,0,1)$ & $1 / 8$ & $(1,1,1)$ & $(0,0,5)$ & $9 / 8$ & $(1,1,5)$ \\
$(0,0,2)$ & $1 / 2$ & $(1,1,2)$ & $(0,0,6)$ & $1 / 2$ & $(1,1,6)$ \\
$(0,0,3)$ & $9 / 8$ & $(1,1,3)$ & $(0,0,7)$ & $1 / 8$ & $(1,1,7)$
\end{tabular}

This corresponds to the form $w_{2,3}^{1}$ and thus to the lattice $E_{8} \perp A_{7} \perp U$. 


\begin{tabular}{cccccc}
\multicolumn{7}{c}{$\langle(0,0,4)\rangle$} & & & & \\
$(0,0,0)$ & 0 & $(0,0,4)$ & $(0,0,2)$ & $1 / 2$ & $(0,0,6)$ \\
$(0,1,0)$ & $3 / 2$ & $(0,1,4)$ & $(0,1,2)$ & 0 & $(0,1,6)$ \\
$(1,0,0)$ & $1 / 2$ & $(1,0,4)$ & $(1,0,2)$ & 1 & $(1,0,6)$ \\
$(1,1,0)$ & 0 & $(1,1,4)$ & $(1,1,2)$ & $1 / 2$ & $(1,1,6)$
\end{tabular}

The generators corresponding to $(0,1,0),(1,0,0)$, and $(0,0,2)$ are independent, so this corresponds to the form $\left(w_{2,1}^{1}\right)^{2} \perp w_{2,1}^{-1}$ and thus to the lattice $E_{7} \perp E_{7} \perp A_{1} \perp U$.

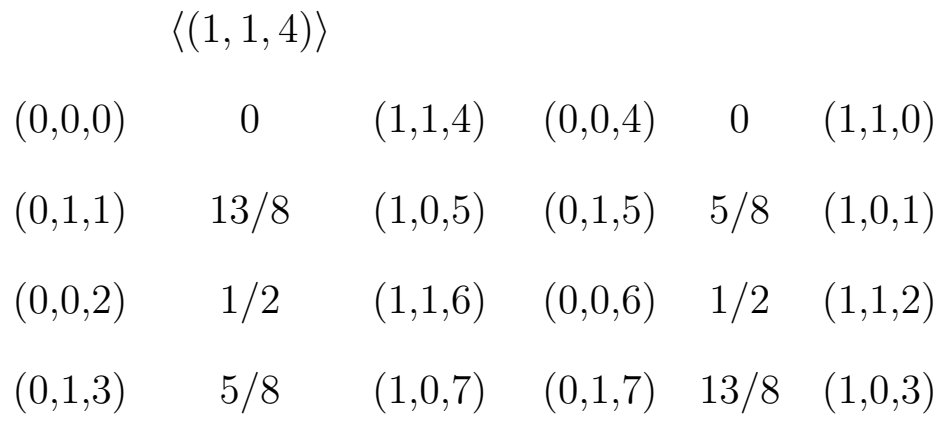

This corresponds to $w_{2,3}^{5}$, which has no representation as a sum of Dynkin lattices.

Now we need to decide how to tell which of these represents the Picard Lattice. The only way to do this is to show that none of the other lattices can be exhibited as a fibration. There are three possibilities for a fibration here: $E_{8} \perp E_{7} \perp U$, $E_{8} \perp A_{7} \perp U$, and $E_{7} \perp E_{7} \perp A_{1} \perp U$. With much pain, one may show that none of these are possible, no matter what kind of curves are added.

Thus, $\operatorname{Pic}(S)=w_{2,3}^{5}$. This lattice has no representation as a sum of Dynkin diagrams. However, we can express this form as $M_{(3,3,4,6),(1,1,1,3),-4}$. 


\section{$3.75(2,4,5,11)$}

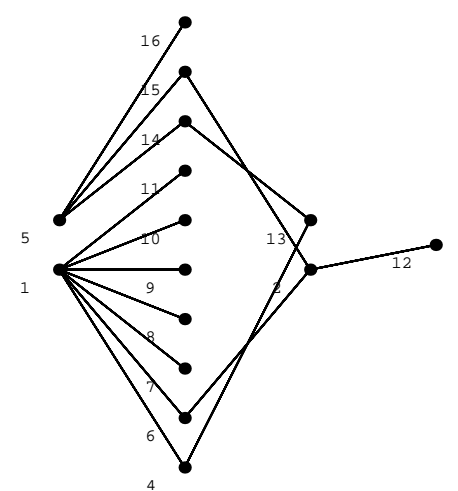

A representative equation for this family of hypersurfaces is $x^{11}+x y^{5}+y^{3} z^{2}+x z^{4}+w^{2}$. The Fibration. Curve 4 has genus 1, and if we consider it as a fibre, then cures 1 and 13 are sections. then curves $2,5,6,12,14-16$ form a $\tilde{D}_{6}$, and curves 7 - 11 may each be completed to an $\tilde{A}_{1}$. As $\rho=13$, this fibration has good rank.

Method: Intermediate Lattice Calculation. The corresponding form is $\left(w_{2,1}^{1}\right)^{2} \perp$ $\left(w_{2,1}^{-1}\right)^{5}$; up to conjugacy, the different elements and values are:

$$
\begin{array}{llllll}
((0,0), 0,0,0,0,0) & 0 & ((0,1), 0,0,0,0,0) & 1 / 2 & ((1,1), 0,0,0,0,0) & 1 \\
((0,0), 0,0,0,0,1) & 3 / 2 & ((0,1), 0,0,0,0,1) & 0 & ((1,1), 0,0,0,0,1) & 1 / 2 \\
((0,0), 0,0,0,1,1) & 1 & ((0,1), 0,0,0,1,1) & 3 / 2 & ((1,1), 0,0,0,1,1) & 0 \\
((0,0), 0,0,1,1,1) & 1 / 2 & ((0,1), 0,0,1,1,1) & 1 & ((1,1), 0,0,1,1,1) & 3 / 2 \\
((0,0), 0,1,1,1,1) & 0 & ((0,1), 0,1,1,1,1) & 1 / 2 & ((1,1), 0,1,1,1,1) & 1 \\
((0,0), 1,1,1,1,1) & 3 / 2 & ((0,1), 1,1,1,1,1) & 0 & ((1,1), 1,1,1,1,1) & 1 / 2
\end{array}
$$

So, up to conjugacy, the distinct isotropic subgroups are $\langle((0,1), 0,0,0,0,1)\rangle$, $\langle((1,1), 0,0,0,1,1)\rangle,\langle((0,0), 0,1,1,1,1)\rangle$, and $\langle((0,1), 1,1,1,1,1)\rangle$. We now view, for each of these, the perpendicular elements arranged by coset. We suppress the zero entries for ease of calculation. 


$$
\begin{array}{ccc}
\multicolumn{4}{c}{\langle((0,1), 0,0,0,0,1)\rangle} \\
((0), 0) & 0 & ((1), 1)
\end{array}
$$

We are left with $w_{2,1}^{1} \perp\left(w_{2,1}^{-1}\right)^{4}$.

$\begin{array}{lcc} & \langle((1,1), 0,0,0,1,1)\rangle & \\ ((0,0), 0,0) & 0 & ((1,1)), 1,1) \\ ((0,0), 1,1) & 1 & ((1,1)), 0,0) \\ ((0,1), 0,1) & 0 & ((1,0)), 1,0) \\ ((1,0), 0,1) & 0 & \end{array}$

This corresponds to $u$, so we now have $u \perp\left(w_{2,1}^{-1}\right)^{3}$. This form is isomorphic to $w_{2,1}^{1} \perp\left(w_{2,1}^{-1}\right)^{4}$

$\begin{array}{lcc} & \langle((0,0), 0,1,1,1,1)\rangle & \\ (0,0,0,0) & 0 & (1,1,1,1) \\ (0,0,1,1) & 1 & (1,1,0,0) \\ (0,1,0,1) & 1 & (1,0,1,0) \\ (1,0,0,1) & 1 & (0,1,1,0)\end{array}$

This corresponds to $v$, so we now have $v \perp\left(w_{2,1}^{1}\right)^{2} \perp w_{2,1}^{-1}$. This form is isomorphic to $w_{2,1}^{1} \perp\left(w_{2,1}^{-1}\right)^{4}$. 


$$
\begin{array}{rccccc}
\langle((0,1), 1,1,1,1,1)\rangle & & & & & \\
((0), 0,0,0,0,0) & 0 & ((1), 1,1,1,1,1) & ((0), 0,1,1,0,0) & 1 & ((1), 1,0,0,1,1) \\
((0), 0,0,0,1,1) & 1 & ((1), 1,1,1,0,0) & ((0), 1,0,1,0,0) & 1 & ((1), 0,1,0,1,1) \\
((0), 0,0,1,0,1) & 1 & ((1), 1,1,0,1,0) & ((0), 1,1,0,0,0) & 1 & ((1), 0,0,1,1,1) \\
((0), 0,1,0,0,1) & 1 & ((1), 1,0,1,1,0) & ((0), 0,1,1,1,1) & 0 & ((1), 1,0,0,0,0) \\
((0), 1,0,0,0,1) & 1 & ((1), 0,1,1,1,0) & ((0), 1,0,1,1,1) & 0 & ((1), 0,1,0,0,0) \\
((0), 0,0,1,1,0) & 1 & ((1), 1,1,0,0,1) & ((0), 1,1,0,1,1) & 0 & ((1), 0,0,1,0,0) \\
((0), 0,1,0,1,0) & 1 & ((1), 1,0,1,0,1) & ((0), 1,1,1,0,1) & 0 & ((1), 0,0,0,1,0) \\
((0), 1,0,0,1,0) & 1 & ((1), 0,1,1,0,1) & ((0), 1,1,1,1,0) & 0 & ((1), 0,0,0,0,1)
\end{array}
$$

This corresponds to $u \perp v$, so we now have $u \perp v \perp w_{2,1}^{1}$. This form is isomorphic to $w_{2,1}^{1} \perp\left(w_{2,1}^{-1}\right)^{4}$.

The Answer. We obtained the same form in each case. As $\rho=13, \operatorname{Pic}(S)=$ $E_{7} \perp\left(A_{1}\right)^{4} \perp U$.

\section{$3.76 \quad(2,5,6,13)$}

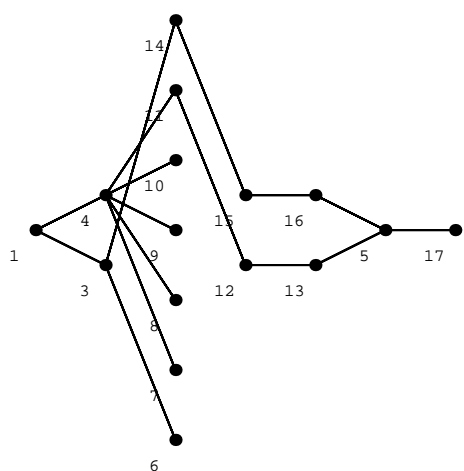

A representative equation for this family of hypersurfaces is $x^{13}+x^{3} y^{4}+y^{4} z+x z^{4}+w^{2}$.

The Fibration. Curve 1, with genus 1, is a fibre; curves 3, 4 are sections, curves 
5, 11 - 17 form an $\tilde{E}_{7}$ and curves 6 - 10 each complete to an $\tilde{A}_{1}$. As $\rho=14$, this fibration has good rank.

Method: Intermediate Lattice Calculation. We did this particular calculation already for number 26 , so see 3.26 . We must choose from the variety of answers.

The Answer. Here is a fibration which reflects one of the possibilities: view curves 4, $8-11$ as a $\tilde{D}_{4}$, so that curves 1 and 7 are 2-sections and curve 12 is a section. Then the remaining curves can only be completed to form a $\tilde{D}_{8}$ so that curves 3 and 13 will have multiplicities 2 and 3 respectively. Thus, $\operatorname{Pic}(S)=D_{8} \perp D_{4} \perp U$.

\section{$3.77 \quad(1,5,7,13)$}

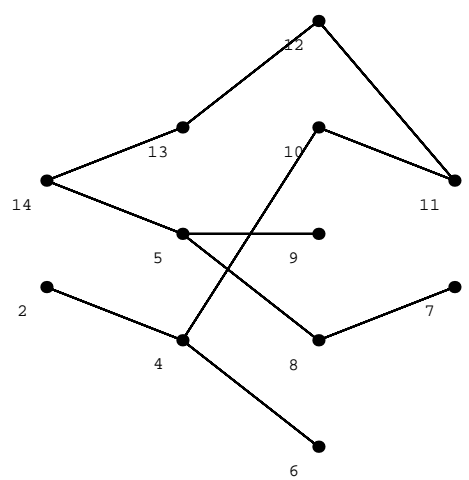

A representative equation for this family of hypersurfaces is $x^{26}+x y^{5}+x^{5} z^{3}+y z^{3}+w^{2}$. Curve 2 has genus 1 . If we consider it as a fibre, then curve 4 is a section. Curves 5 , 7 - 14 form an $\tilde{E}_{8}$, and curve 6 may then be completed to form an $\tilde{A}_{1}$. As $\rho=11$, this fibration has good rank. As we have an $\tilde{E}_{8}$, we know that $M W$ is trivial and so the index of this lattice in $\operatorname{Pic}(S)$ is 1. Therefore, $\operatorname{Pic}(S)=E_{8} \perp A_{1} \perp U$. 


\section{$3.78(1,4,6,11)$}

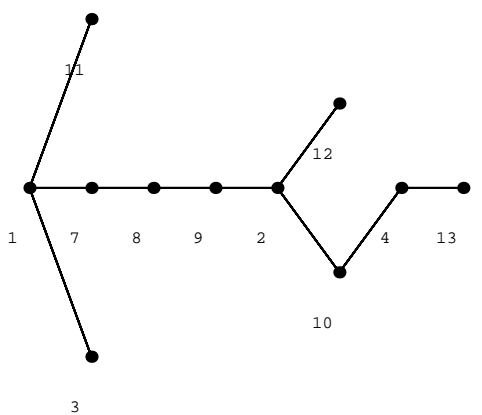

A representative equation for this family of hypersurfaces is $x^{22}+x^{2} 3 y^{5}+y^{4} z+x^{4} z^{3}+$ $y z^{3}+w^{2}$.

This is one of Arnold's 14 original surface singularities. $\rho=10$ and curve 3 has genus 1. If we consider curve 3 to be a fibre, then curve 1 is a section and we are left with $\tilde{E}_{7}$ and $\tilde{A}_{1}$. This fibration has good rank, but the associated form has an isotropic subgroup. However, there are at most 2 sections as the discriminant groups of $\tilde{E}_{7}$ and $\tilde{A}_{1}$ are $\mathbb{Z}_{2}$. If there are 2 sections, then $\operatorname{disc}(\operatorname{Pic}(S))=1$ and the only unimodular lattice of rank 10 is $E_{8} \perp U$...but this only has one section. Contradiction! Therefore $\operatorname{Pic}(S)=E_{7} \perp A_{1} \perp U$

\section{$3.79 \quad(2,5,9,16)$}

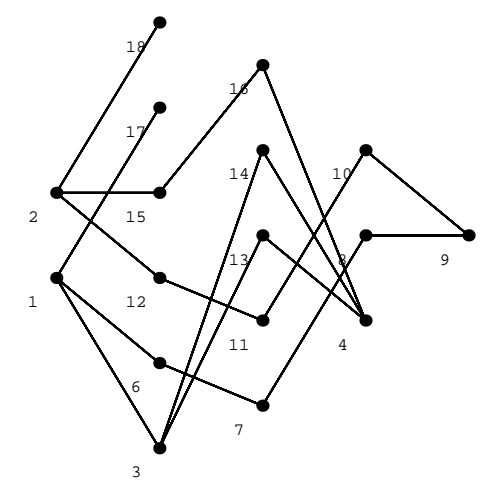


A representative equation for this family of hypersurfaces is $x^{16}+x y^{6}+x^{7} z^{2}+y z^{3}+w^{2}$. Curve 4 has genus 2 and $\rho=15$. We can see curves $2,8-12,15,16,18$ as forming an $\tilde{E}_{8}$, so that curve 7 is a section and curve 4 is a 2 -section, and then the remaining curves form a $\tilde{D}_{5}$. This fibration has good rank. As we have an $\tilde{E}_{8}$, we know that $M W$ is trivial and so the index of this lattice in $\operatorname{Pic}(S)$ is 1 . Therefore, $\operatorname{Pic}(S)=E_{8} \perp D_{5} \perp U$.

\section{$3.80(4,5,13,22)$}

A representative equation for this family of hypersurfaces is $x^{11}+x y^{8}+y z^{3}+w^{2}$. See (3.46). They have the same rank and configuration of curves.

\section{$3.81(2,3,8,13)$}

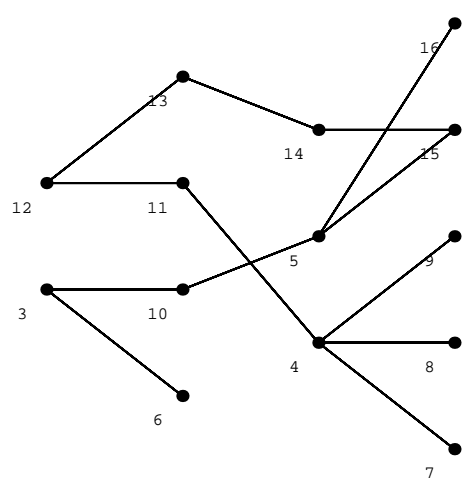

A representative equation for this family of hypersurfaces is $x^{13}+x y^{8}+y^{6} z+x z^{3}+w^{2}$. There are no curves of genus 1 . We may, however, see curves 3, 5, 10 - 16 as forming an $\tilde{E}_{8}$, so that curve 4 is a section and each of curves 7 - 9 may be completed to form an $\tilde{A}_{1}$. As we have an $\tilde{E}_{8}$, we know that $M W$ is trivial and so the index of this lattice in $\operatorname{Pic}(S)$ is 1. Therefore, $\operatorname{Pic}(S)=E_{8} \perp\left(A_{1}\right)^{3} \perp U$. 


\section{$3.82(1,3,7,11)$}

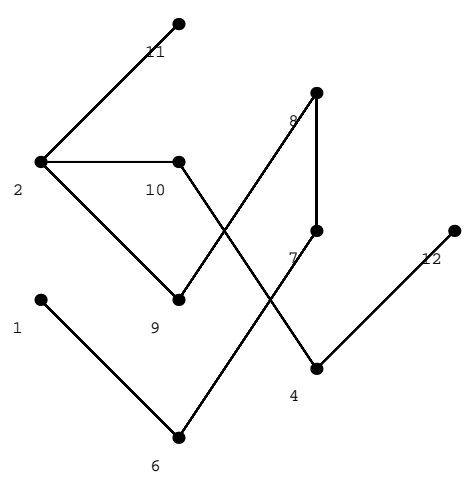

A representative equation for this family of hypersurfaces is $x^{22}+x y^{7}+y^{5} z+x z^{3}+w^{2}$. Curve 1 has genus 1; if we consider it as a fibre, then curve 6 is a section and the remaining curves form an $\tilde{E}_{7}$. As $\rho=9$, this fibration has good rank. This lattice has discriminant 2, which is square-free and thus it has index 1 in $\operatorname{Pic}(S)$. Thus $\operatorname{Pic}(S)=E_{7} \perp U$

\section{$3.83(4,5,18,27)$}

A representative equation for this family of hypersurfaces is $x^{11} y^{2}+x y^{10}+x^{9} z+$ $z^{3}+w^{2}$

See section 3.68. Number 68 has the same rank and configuration of curves. 


\section{$3.84(5,6,7,9)$}

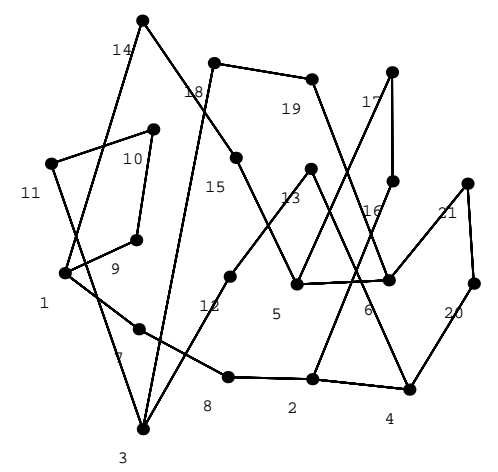

A representative equation for this family of hypersurfaces is $x^{3} y^{2}+x^{4} z+y z^{3}+y^{3} w+w^{3}$.

The Fibration. There are no curves of genus 1 . However, we may see curves 1, 7

- 10, 14, 15 as an $\tilde{E}_{6}$, so that curves $2,5,11$ are sections, curves $3,4,6,12,13,18$ -

21 form an $\tilde{A}_{8}$, and curves 16,17 may be completed to form an $\tilde{A}_{2}$. As $\rho=18$, this fibration has good rank.

Method: Intermediate Lattice Calculation. The form corresponding to this fibration is $w_{3,1}^{-1} \perp w_{3,1}^{1} \perp w_{3,2}^{1}$. Here are the elements and values:

\begin{tabular}{|ccc|rcr|}
\hline$(0,0,0)$ & 0 & & $(0,1,0)$ & $4 / 3$ & $(0,2,0)$ \\
$(1,0,0)$ & $2 / 3$ & $(2,0,0)(1,1,0)$ & 0 & $(1,2,0),(2,1,0),(2,2,0)$ & \\
$(0,0,1)$ & $4 / 9$ & & $(0,1,1)$ & $16 / 9$ & $(0,2,1)$ \\
$(1,0,1)$ & $10 / 9$ & $(2,0,1)(1,1,1)$ & $4 / 9$ & $(1,2,1),(2,1,1),(2,2,1)$ & \\
\hline \hline$(0,0,2)$ & $16 / 9$ & & $(0,1,2)$ & $10 / 9$ & $(0,2,2)$ \\
$(1,0,2)$ & $4 / 9$ & $(2,0,2)(1,1,2)$ & $16 / 9$ & $(1,2,2),(2,1,2),(2,2,2)$ & \\
$(0,0,3)$ & 0 & & $(0,1,3)$ & $4 / 3$ & $(0,2,3)$ \\
$(1,0,3)$ & $2 / 3$ & $(2,0,3)(1,1,3)$ & 0 & $(1,2,3),(2,1,3),(2,2,3)$ & \\
\hline
\end{tabular}




\begin{tabular}{|c|c|c|c|c|c|}
\hline$(0,0,4)$ & $10 / 9$ & & $(0,1,4)$ & $4 / 9$ & $(0,2,4)$ \\
\hline$(1,0,4)$ & $16 / 9$ & $(2,0,4)(1,1,4)$ & $10 / 9$ & $(1,2,4),(2,1,4),(2,2,4)$ & \\
\hline$(0,0,5)$ & $10 / 9$ & & $(0,1,5)$ & $4 / 9$ & $(0,2,5)$ \\
\hline$(1,0,5)$ & $16 / 9$ & $(2,0,5)(1,1,5)$ & $10 / 9$ & $(1,2,5),(2,1,5),(2,2,5)$ & \\
\hline$(0,0,6)$ & 0 & & $(0,1,6)$ & $4 / 3$ & $(0,2,6)$ \\
\hline$(1,0,6)$ & $2 / 3$ & $(2,0,6)(1,1,6)$ & 0 & $(1,2,6),(2,1,6),(2,2,6)$ & \\
\hline$(0,0,7)$ & $16 / 9$ & & $(0,1,7)$ & $10 / 9$ & $(0,2,7)$ \\
\hline$(1,0,7)$ & $4 / 9$ & $(2,0,7)(1,1,7)$ & $16 / 9$ & $(1,2,7),(2,1,7),(2,2,7)$ & \\
\hline$(0,0,8)$ & $4 / 9$ & & $(0,1,8)$ & $16 / 9$ & $(0,2,8)$ \\
\hline$(1,0,8)$ & $10 / 9$ & $(2,0,8)(1,1,8)$ & $4 / 9$ & $(1,2,8),(2,1,8),(2,2,8)$ & \\
\hline
\end{tabular}

There are three isotropic subgroups, $\langle(1,1,0)\rangle,\langle(0,0,3)\rangle$, and $\langle(1,1,3)\rangle$. In dealing with the first two of these, we will omit the last and first two entries respectively, in order to simplify the calculation. First, the reader may notice that the elements perpendicular to $\langle(1,1,0)\rangle=\langle(1,1)\rangle$ are exactly $(0,0),(1,1),(2,2)$, all of which have value 0 . Thus, we are left with $w_{3,2}^{1}$, which corresponds to the lattice $E_{8} \perp A_{8} \perp U$. Next, the reader may notice that the elements perpendicular to $\langle(0,0,3)\rangle=\langle(3)\rangle$ are exactly (0), (3), (6), all of which have value 0 . Thus, we are left with $w_{3,1}^{-1} \perp w_{3,1}^{1}$, which corresponds to $E_{8} \perp E_{6} \perp A_{2} \perp U$. Finally, we have the elements perpendicular to $\langle(1,1,3)\rangle$ arranged by coset:

\begin{tabular}{|c|c|c|c|c|c|c|c|}
\hline 0 & $(0,0,0)$ & $(1,1,3)$ & $(2,2,6)$ & & & & \\
\hline 0 & $(0,0,3)$ & $(1,1,6)$ & & $16 / 9$ & $(0,1,8)$ & $(1,2,2)$ & $(2,0,5)$ \\
\hline 0 & $(0,0,6)$ & $(1,1,0)$ & $(2,2,3)$ & $16 / 9$ & $(0,2,1)$ & $(1,0,4)$ & $(2,1,7)$ \\
\hline $10 / 9$ & $(0,1,2)$ & $(1,2,5)$ & $(2,0,8)$ & $4 / 9$ & $(0,2,4)$ & $(1,0,7)$ & $(2,1,1)$ \\
\hline $4 / 9$ & $(0,1,5)$ & $(1,2,8)$ & $(2,0,2)$ & $10 / 9$ & $(0,2,7)$ & $(1,0,1)$ & $(2,1,4)$ \\
\hline
\end{tabular}

This is the form $w_{3,2}^{1}$, which corresponds to the lattice $E_{8} \perp A_{8} \perp U$. 
Now we need to determine which of these is the correct lattice. The only way to do this is to (a) exhibit a fibration corresponding to one of the forms and (b) show that none of the other lattices can be exhibited as a fibration. There are two possibilities for a fibration here: $E_{8} \perp E_{6} \perp A_{2} \perp U$, and $E_{8} \perp A_{8} \perp U$. With much pain, one may show that the first is not possible, no matter what kind of curves are added. We may see the last one in the following way.

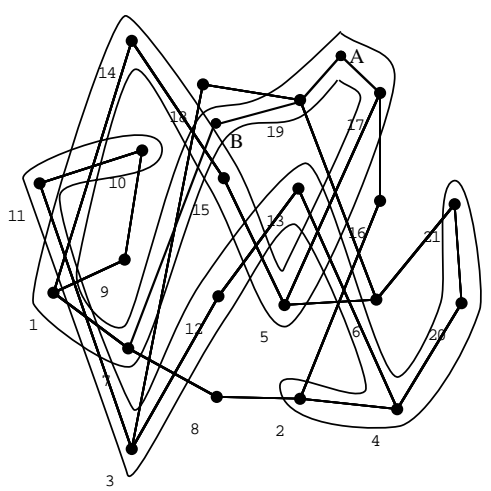

We can see curves $2-4,10-13,20,21$ as an $\tilde{E}_{8}$, so that curve 9 is a section, curve 6 is a 2-section, and curves 8, 16, 18 are 3-sections. We may now complete curves 1, 5, 7, $14,15,17,19$ to form an $\tilde{A}_{8}$ by adding curves A and B. Thus, $\operatorname{Pic}(S)=E_{8} \perp A_{8} \perp U$.

\section{$3.85(2,3,4,5)$}

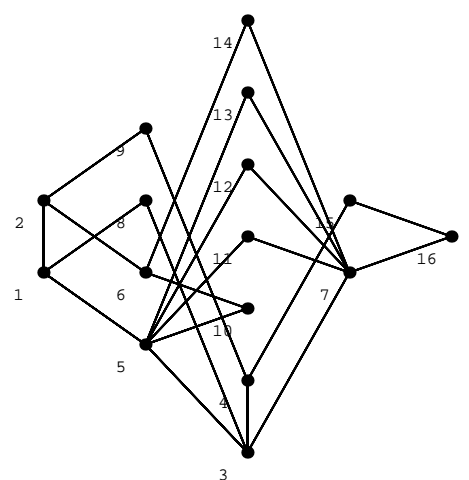

A representative equation for this family of hypersurfaces is $x^{7}++x y^{4}+y^{2} z^{2}+x z^{3}+$ $y^{3} w+x^{2} w^{2}+z w^{2}$. 
Curves 3 and 5 each have genus 1 , and they intersect with multiplicity 2 . If we consider either of these as a fibre, we will have a nightmare, so instead we will look for another fibration beginning. It is easy to see curves $1,11-14$ as forming a $\tilde{D}_{4}$, so that curve 6 is a section, curves 3 and 16 are 2 -sections, and curve 5 is a 3 -section. Then we may complete curve 10 to an $\tilde{A}_{1}$ and the remaining curves to an $\tilde{A}_{6}$. As $\rho=13$, this fibration has good rank. As the discriminants of these fibres are coprime, we know that $M W$ is trivial (it must embed in the discriminant group of each fibre). Therefore $\operatorname{Pic}(S)=D_{4} \perp A_{6} \perp A_{1} \perp U$.

\section{$3.86(4,5,7,9)$}

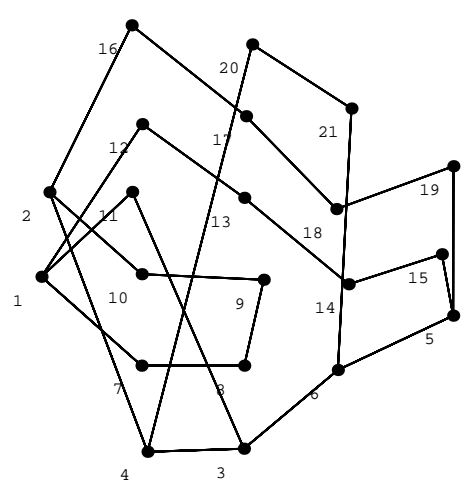

A representative equation for this family of hypersurfaces is $x^{5} y+y^{5}+x z^{3}+x^{4} w+z w^{2}$. The Fibration. Curve 3 has genus 1. If we consider it as a fibre, then curves 4, 6 , 11 are sections, curves 1, 2, 5, $7-10,12-19$ form an $\tilde{A}_{14}$, and curves 20, 21 may be completed to form an $\tilde{A}_{2}$. As $\rho=18$, this fibration has good rank.

Method: Intermediate Lattice Calculation. The discriminant group for this fibration is $\mathbb{Z}_{15} \oplus \mathbb{Z}_{3}$. We will use Miranda's notation. Here is a table of elements and values: 


$$
\begin{array}{lllllllllll}
(0,0) & 0 & (0,1) & -1 / 3 & (0,2) & & & & & \\
(1,0) & -7 / 15 & (1,1) & -4 / 5 & (1,2) & (8,0) & -13 / 15 & (8,1) & -1 / 5 & (8,2) \\
(2,0) & -13 / 15 & (2,1) & -1 / 5 & (2,2) & (9,0) & -4 / 5 & (9,1) & -2 / 15 & (9,2) \\
(3,0) & -1 / 5 & (3,1) & -8 / 15 & (3,2) & (10,0) & -2 / 3 & (10,1) & 0 & (10,2) \\
(4,0) & -7 / 15 & (4,1) & -4 / 5 & (4,2) & (11,0) & -7 / 15 & (11,1) & -4 / 5 & (11,2) \\
(5,0) & -2 / 3 & (5,1) & 0 & (5,2) & (12,0) & -1 / 5 & (12,1) & -8 / 15 & (12,2) \\
(6,0) & -4 / 5 & (6,1) & -2 / 15 & (6,2) & (13,0) & -13 / 15 & (13,1) & -1 / 5 & (13,2) \\
(7,0) & -13 / 15 & (7,1) & -1 / 5 & (7,2) & (14,0) & -7 / 15 & (14,1) & -4 / 5 & (14,2)
\end{array}
$$

The only isotropic subgroup is generated by $\langle(5,1)\rangle$. If we look at the perpendicular elements arranged by coset, we see...

$$
\begin{array}{llll}
0 & (0,0) & (5,1) & (10,2) \\
-1 / 5 & (3,0) & (8,1) & (13,2) \\
-4 / 5 & (6,0) & (11,1) & (1,2) \\
-4 / 5 & (9,0) & (14,1) & (4,2) \\
-1 / 5 & (12,0) & (2,1) & (7,2)
\end{array}
$$

...that this corresponds to the form $w_{5,1}^{-1}$, which is the lattice $E_{8} \perp T_{2,5,5}$.

\section{$3.87(1,3,4,5)$}

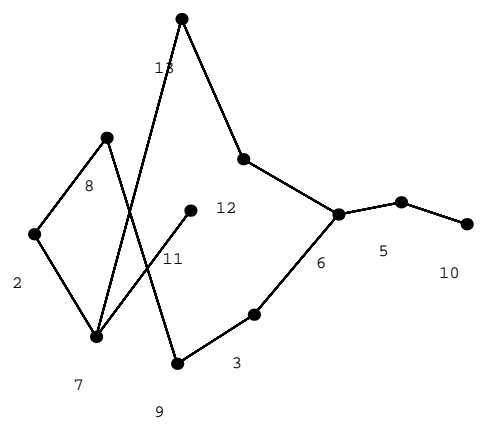


A representative equation for this family of hypersurfaces is $x^{13}+x y^{4}+y^{3} z+x z^{3}+$ $z^{2} w+x^{3} w^{2}+y w^{2}$

This is one of Arnold's 14 original surface singularities. $\rho=10$. Curve 2 has genus 1. Note that aside from curve 2 , we have a $T_{3,4,5}$. This lattice has no representation as a sum of Dynkin lattices.

\section{$3.88 \quad(2,5,9,11)$}

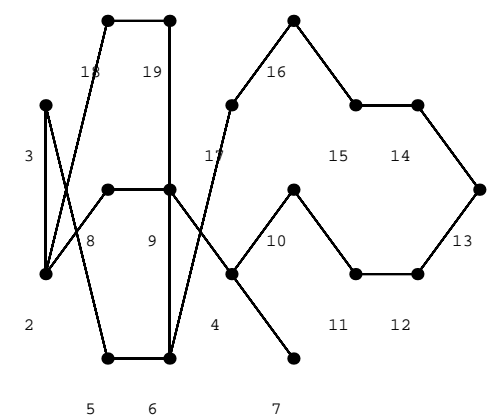

A representative equation for this family of hypersurfaces is $x^{11} y+x y^{5}+x^{9} z+z^{3}+$ $x^{8} w+y w^{2}$.

Curve 2 has genus 1 and $\rho=16$. We can see curves $4,7-14$ as an $\tilde{E}_{8}$, so that curve 15 is a section and curve 2 is a 2 -section. Then, the remaining curves form an $\tilde{E}_{6}$. This fibration satisfies the Shioda-Tate formula with $r k(M W)=0$. And, because we have an $\tilde{E}_{8}$, the index of this lattice in $\operatorname{Pic}(S)$ is 1 . Thus, $\operatorname{Pic}(S)=E_{8} \perp E_{6} \perp U$. 


\section{$3.89(1,2,3,5)$}

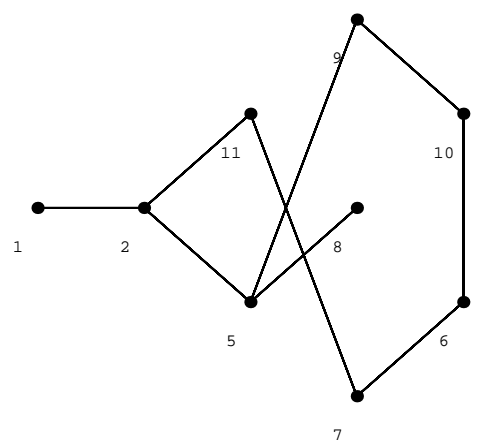

A representative equation for this family of hypersurfaces is $x^{11}+x y^{5}+y^{4} z+x^{2} z^{3}+$ $y z^{3}+y^{3} w+z^{2} w+x w^{2}$.

Curve 6 has genus $1 ; \rho=8$ and there is no fibration with good rank. Thus, we must look at the matrix. It has discriminant 11, and corresponds to $w_{11,1}^{-1}$. Because 11 is square-free, the lattice has index 1 in $\operatorname{Pic}(S)$ and is thus equal to $\operatorname{Pic}(S)$. This lattice has no representation as a sum of Dynkin lattices. However, we can express this form as $M_{(1,2,4),(1,1,2),-2}$.

\section{$3.90(4,6,7,17)$}

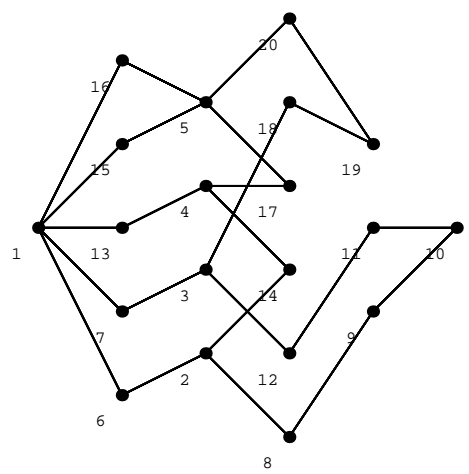

A representative equation for this family of hypersurfaces is $x^{7} y+x y^{5}+x^{5} z^{2}+y z^{4}+w^{2}$.

Curve 3 has genus 1 . Consider curves $3,7-12,18,19$ as an $\tilde{E}_{8}$. Then curve 2 is a 
section, curve 20 is a 2-section, and curve 1 is a 3 -section. Curves 4, 5, 13 - 17 form a $\tilde{D}_{6}$, and curve 6 can be completed to form an $\tilde{A}_{1}$. As $\rho=17$, this fibration has good rank, and because we have an $\tilde{E}_{8}$, the index of this lattice in $\operatorname{Pic}(S)$ is 1 . Thus, $\operatorname{Pic}(S)=E_{8} \perp D_{6} \perp A_{1} \perp U$.

\section{$3.91 \quad(5,6,8,19)$}

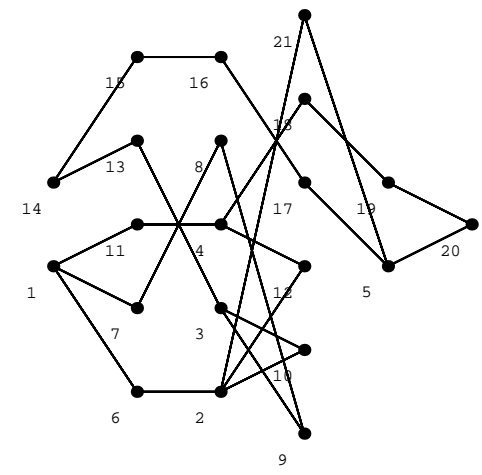

A representative equation for this family of hypersurfaces is $x^{4} y^{3}+x^{6} z+y^{5} z+y z^{4}+w^{2}$. Curve 2 has genus 1 . We can consider curves $3,3,8-10,13-17$ as an $\tilde{E}_{8}$, so that curve 5 is a section, curve 7 is a 2 -section, an curve 2 is a 3 -section. Then curves $1,4,6,11,12,18-20$ form an $\tilde{E}_{7}$, and curve 21 can be completed to form an $\tilde{A}_{1}$. As $\rho=18$, this fibration has good rank. Because we have an $\tilde{E}_{8}$, the index of this lattice in $\operatorname{Pic}(S)$ is 1 . Thus, $\operatorname{Pic}(S)=E_{8} \perp E_{7} \perp A_{1} \perp U$.

\section{$3.92 \quad(3,5,11,19)$}

A representative equation for this family of hypersurfaces is $x^{11} y+x y^{7}+x^{9} z+$ $y z^{3}+w^{2}$

See section 3.68. Number 68 has the same rank and configuration of curves. 


\section{$3.93(3,4,10,17)$}

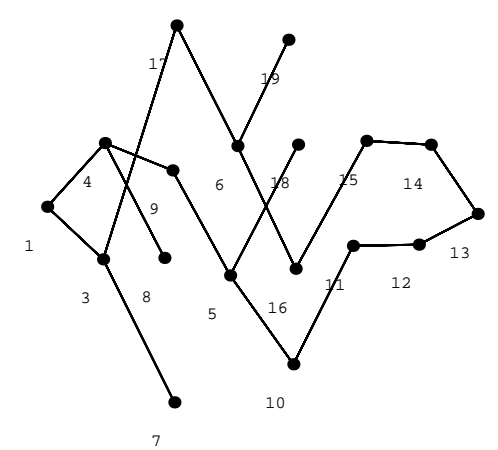

A representative equation for this family of hypersurfaces is $x^{10} y+x^{2} y^{7}+x^{8} z+y^{6} z+$ $y z^{3}+w^{2}$

The Fibration. Curve 2 has genus 1 and if we consider it as a fibre, then curves 3 and 4 are sections. All remaining curves except curve 7 and 8 form a $\tilde{D}_{12}$, and curves 7 and 8 can each be completed to form an $\tilde{A}_{1}$. As $\rho=16$, this fibration has good rank.

Method: Intermediate Lattice Calculation. The associated form is $v \perp\left(w_{2,1}^{-1}\right)^{2}$. Here are elements of the discriminant group and their values:

$$
\begin{array}{lllllll}
((0,0), 0,0) & 0 & ((0,0), 0,1) & 3 / 2 & ((0,0), 1,0) & ((0,0), 1,1) & 1 \\
((1,0), 0,0) & 1 & ((1,0), 0,1) & 1 / 2 & ((1,0), 1,0) & ((1,0), 1,1) & 0 \\
((0,1), 0,0) & 1 & ((0,1), 0,1) & 1 / 2 & ((0,1), 1,0) & ((0,1), 1,1) & 0 \\
((1,1), 0,0) & 1 & ((1,1), 0,1) & 1 / 2 & ((1,1), 1,0) & ((1,1), 1,1) & 0
\end{array}
$$

There are three conjugate isotropic subgroups, generated by $\langle((1,1), 1,1)\rangle$, $\langle((0,1), 1,1)\rangle$, and $\langle((1,0), 1,1)\rangle$. We will choose to work with the first of these. The perpendicular elements, arranged by coset, are 


$$
\begin{array}{lcl}
((0,0), 0,0) & 0 & ((1,1), 1,1) \\
((0,0), 1,1) & 1 & ((1,1), 0,0) \\
((1,0), 0,1) & 1 / 2 & ((0,1), 1,0) \\
((1,0), 1,0) & 1 / 2 & ((0,1), 0,1)
\end{array}
$$

This corresponds to the form $\left(w_{2,1}^{1}\right)^{2}$, so $\operatorname{Pic}(S)=E_{8} \perp D_{6} \perp U$.

\section{$3.94(3,4,5,7)$}

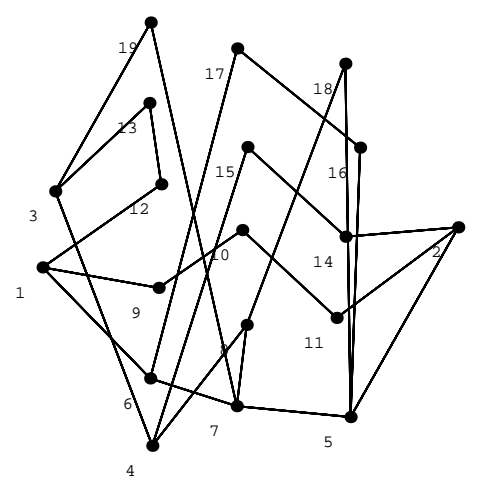

A representative equation for this family of hypersurfaces is $x^{5} y+x y^{4}+x^{3} z^{2}+y z^{3}+$ $x^{4} w+y^{3} w+z w^{2}$.

Curve 7 has genus 1; there is no fibration with good rank. The matrix has discriminant 19; as this is square-free, the lattice has index 1 in $\operatorname{Pic}(S)$. The form is $w_{19,1}^{-1}$; $\rho=16$ and this cannot be represented as a sum of Dynkin lattices. However, we can express this form as $M_{(2,3,4,6),(1,1,2,2),-4}$. 


\section{$3.95 \quad(2,3,5,7)$}

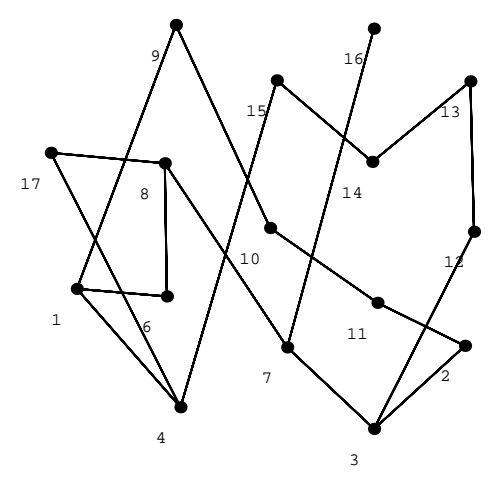

A representative equation for this family of hypersurfaces is $x^{7} y+x y^{5}+x^{6} z+y^{4} z+$ $x z^{3}+x^{5} w+z^{2} w+y w^{2}$.

Curve 8 has genus 1; there is no fibration with good rank. The matrix has discriminant 17; as this is square-free, the corresponding lattice has index 1 in $\operatorname{Pic}(S)$. The form is $w_{17,1}^{-1} ; \rho=14$ and this cannot be represented as a sum of Dynkin lattices. However, we can express this form as $M_{(1,2,4,6),(1,1,2,3),-4}$. 


\section{CHAPTER IV}

\section{$\operatorname{Pic}(S)$ versus $\operatorname{Pic}(\mathbf{J}(S))$}

This chapter is an extension of Section 1.8.4.

4.0.0.2 Definition. A vector bundle $E$ over $S$ is simple if $\operatorname{Ext}_{\mathcal{O}_{S}}^{0}(E, E)=\operatorname{End}_{\mathcal{O}_{S}}(E)=\mathbb{C}$

4.0.0.3 Definition. A bundle $E$ is stable with respect to an ample divisor $H$ if

$$
\frac{c_{1}(B) \cdot H}{r k(B)} \leq \frac{c_{1}(E) \cdot H}{r k(E)}
$$

for any subsheaf $B$ of $E$ with $r k(B)<r k(E)$, and if the equality holds, then

$$
\frac{c_{1}^{2}(B)-2 c_{2}(B)-c_{1}(B) \cdot K}{2 r k(B)}<\frac{c_{1}^{2}(E)-2 c_{2}(E)-c_{1}(E) \cdot K}{2 r k(E)} .
$$

4.0.0.4 Definition. A bundle $E$ is $\mu$-stable (resp. $\mu$-semi-stable) if

$$
\frac{c_{1}(B) \cdot H}{r k(B)}<\frac{c_{1}(E) \cdot H}{r k(E)} \quad\left(\operatorname{resp} \cdot \frac{c_{1}(B) \cdot H}{r k(B)} \leq \frac{c_{1}(E) \cdot H}{r k(E)}\right)
$$

for any subsheaf $B$ of $E$ with $r k(B)<r k(E)$.

\subsection{Moduli Spaces of Vector Bundles}

4.1.0.5 Definition. Define the extended K3 lattice $\tilde{H}(S, \mathbb{Z})=H^{0}(S, \mathbb{Z}) \oplus H^{2}(S, \mathbb{Z}) \oplus$ $H^{4}(S, \mathbb{Z})$ 
Notice that $H^{0}(S, \mathbb{Z}) \cong \mathbb{Z}$ because $H^{0}$ counts connected components and $H^{4}(S, \mathbb{Z}) \cong$ $\mathbb{Z}$ because $S$ is compact.

4.1.0.6 Remark. There is a natural Hodge structure on $\tilde{H}(S, \mathbb{Z})$ obtained by restricting the Hodge structure on $\tilde{H}(S, \mathbb{C})=\tilde{H}(S, \mathbb{Z}) \oplus \mathbb{C}$. In this way, $H^{2}(S, \mathbb{Z})$ is both a sublattice and a Hodge substructure of $\tilde{H}(S, \mathbb{Z})$.

4.1.0.7 Definition. Elements of the extended K3 lattice are denoted $(r, l, s)$ and for a vector bundle $E$ on $S$ we associate a vector $v$ to $E$ by $r=r k(E), l=c_{1}(E)$, and $s=r k(E)+\frac{1}{2} c_{1}^{2}(E)-c_{2}(E)$.

4.1.0.8 Definition. We extend the inner product on $H^{2}(S, \mathbb{Z})$ to $\tilde{H}(S, \mathbb{Z})$ as follows: $v^{2}=c_{1}^{2}-2 r s$ (and more generally, $v \cdot v^{\prime}=l \cdot l^{\prime}-r s^{\prime}-r^{\prime} s$ ).

We can now talk about various moduli spaces of vector bundles with $v(E)=v$. The moduli space on which we will focus is $M_{H}(v)$, the moduli space of $H$-stable vector bundles $E$ with $v(E)=v$, where $H$ is an ample divisor on $S$.

Recall that we are looking for a map between $\operatorname{Pic}(S)$ and $\operatorname{Pic}(\mathbf{J}(S))$. We will see that if we can satisfy the hypotheses of [Mukai2, Proposition 6.4], we can use it to obtain such a map. These hypotheses are:

- $v$ is primitive and isotropic.

- $M_{H}(v)$ is nonempty and compact.

The reason for the first condition is that if nonempty, $M_{H}(v)$ has dimension $v^{2}+2$ [Mukai1], so $v$ must be isotropic.

To use the Proposition for our purposes, we also need

- $\mathbf{J}(S) \cong M_{H}(v)$. This will be shown in 4.3.

4.1.0.9 Proof that $v=(d, F, 0)$ is primitive and isotropic. A vector $v \in$ $\tilde{H}^{1,1}(S, \mathbb{Z})$ is primitive if $\tilde{H}^{1,1}(S, \mathbb{Z}) / \mathbb{Z} \cdot v$ has no torsion. Our vector is $(d, F, 0)$ so 
$\tilde{H}^{1,1}(S, \mathbb{Z}) / \mathbb{Z} \cdot v$ having no torsion is equivalent to saying that there exists no vector $(a, C, b) \notin\langle v\rangle$ where $k(a, C, b) \in\langle v\rangle$. If such a vector existed, then $k C=k^{\prime} F$ so that

$F=\frac{k}{k^{\prime}} C$. If $\frac{k}{k^{\prime}} \leq 1$ then $(a, C, b) \in\langle v\rangle$, contradicting our assumption, and if $\frac{k}{k^{\prime}}>1$ then this implies that $F$ is a multiple fibre (and K3 surfaces have none).

To be clearer, note that linear equivalence is $\operatorname{Pic}(S)$-equivalence and numerical equivalence is $H^{2}$-equivalence; because $\operatorname{Pic}(S)$ injects into $H^{2}$, if two elements of $\operatorname{Pic}(S)$ are numerically equivalent, then they are also linearly equivalent. So if $F$ is numerically equivalent to a multiple of $C$, then it is linearly equivalent to a multiple of $C$, and the only things a fibre is linearly equivalent to are other fibres, so this means the multiple of $C$ is a fibre and thus there's a multiple fibre.

It's easier to show that $v=(d, F, 0)$ is isotropic: $v^{2}=(d, F, 0)^{2}=F^{2}-2 d \cdot 0=0$.

\subsection{Construction of Vector Bundles}

We aim to show that we can construct a vector bundle from a minimal index multisection, so that $\mathbf{J}(S)$ will be isomorphic to $M_{H}(d, F, 0)$. We take the intersection of a minimal index $(=d)$ multisection with the generic fibre, and restrict to any fibre; this intersection is a group of points, or a zero-cycle $\xi$ on the surface. [Tyurin] gives us the construction we need. Denote by $\mathcal{I}_{\xi}$ the ideal sheaf corresponding to the cycle $\xi$, and by $\mathcal{I}_{\xi}(F)$ the ideal sheaf twisted by $\mathcal{O}_{S}(F)$.

4.2.0.10 Theorem. Let $F$ be a fibre of a $\mathrm{K} 3$ elliptic surface $S$. Let $\xi \subset F$ be a 0 -cycle of degree $d>1$. Then there exists a vector bundle $E(\xi, F)$ defined by the non-split extension

$$
0 \rightarrow H^{1}\left(\mathcal{I}_{\xi}(F)\right) \otimes \mathcal{O}_{S} \rightarrow E(\xi, F) \rightarrow \mathcal{I}_{\xi}(F) \rightarrow 0
$$


Proof of 4.2.0.10. We need to show that $h^{1}\left(\mathcal{I}_{\xi}(F)\right)>0$ so that the extension makes sense. First we will show that $h^{1}\left(\mathcal{O}_{S}(F)\right)=0$; we use Riemann-Roch on $S$. $H^{0}\left(\mathcal{O}_{S}(F)\right)$ is the space of divisors on the surface equivalent to the fibre. This has projective dimension 1 , or linear dimension 2. $h^{2}\left(\mathcal{O}_{S}(F)\right)=h^{0}\left(\mathcal{O}_{S}(-F)\right)=0$ as $F$ is effective. Then Riemann- $\operatorname{Roch} \operatorname{reads} h^{1}\left(\mathcal{O}_{S}(F)\right)=2+0-F^{2} / 2-2=0$.

Now we will use the fact that $h^{1}\left(\mathcal{O}_{S}(F)\right)=0$ in the proof that $h^{1}\left(\mathcal{I}_{\xi}(F)\right)=d-1$. Begin with the exact sequence

$$
0 \rightarrow \mathcal{I}_{\xi} \rightarrow \mathcal{O}_{S} \rightarrow \mathcal{O}_{\xi} \rightarrow 0
$$

and tensor by $\mathcal{O}_{S}(F)$ to get

$$
0 \rightarrow \mathcal{I}_{\xi}(F) \rightarrow \mathcal{O}_{S}(F) \rightarrow \mathcal{O}_{\xi} \otimes \mathcal{L}(F) \rightarrow 0
$$

A dimension count on the long exact cohomology sequence shows that $H^{1}\left(\mathcal{I}_{\xi}(F)\right)$ has $\operatorname{dim}=d-1$ for any degree $d$ cycle $\xi \subset F$.

4.2.0.11 Remark. Notice that $H^{0}\left(\mathcal{O}_{S}(F)\right)$ and $H^{1}\left(\mathcal{O}_{S}(F)\right)$ are independent of the cycle, so we know also that if we have any cycle of degree $d$ with $h^{1}\left(\mathcal{I}_{\xi}(F)\right)=d-1$, then it is contained in $F$.

Now note, using Serre Duality, that

$$
\operatorname{Ext}_{\mathcal{O}_{S}}^{1}\left(\mathcal{I}_{\xi}(F), \omega_{S}\right)=\left[\operatorname{Ext}_{\mathcal{O}_{S}}^{1}\left(\mathcal{O}_{S}, \mathcal{I}_{\xi}(F)\right)\right]^{*}=H^{1}\left(\mathcal{I}_{\xi}(F)\right)^{*}=\operatorname{Hom}\left(H^{1}\left(\mathcal{I}_{\xi}(F)\right), \mathcal{O}_{S}\right)
$$

Therefore,

$$
\operatorname{Ext}_{\mathcal{O}_{S}}^{1}\left(\mathcal{I}_{\xi}(F), H^{1}\left(\mathcal{I}_{\xi}(F)\right)\right)=\operatorname{Hom}\left(H^{1}\left(\mathcal{I}_{\xi}(F)\right), H^{1}\left(\mathcal{I}_{\xi}(F)\right)\right)
$$

If we look at the identity element in $\operatorname{Hom}\left(H^{1}\left(\mathcal{I}_{\xi}(F)\right), H^{1}\left(\mathcal{I}_{\xi}(F)\right)\right)$ we may view it as the cocycle defining the extension

$$
0 \rightarrow H^{1}\left(\mathcal{I}_{\xi}(F)\right) \rightarrow E(\xi, F) \rightarrow \mathcal{I}_{\xi}(F) \rightarrow 0 \quad(\text { see }[\text { Tyurin }])
$$


It remains to show that $E(\xi, F)$ is locally free (and thus a vector bundle). [Tyurin, Lemma 1.2 and Corollary 1] gives a criterion: $E(\xi, F)$ is locally free if for every subcycle $\xi^{\prime} \subset \xi, h^{1}\left(\mathcal{I}_{\xi^{\prime}}(F)\right)<h^{1}\left(\mathcal{I}_{\xi}(F)\right)$. We now show this. We examine the cohomology exact sequence [Tyurin, 1.9]:

$$
0 \rightarrow H^{0}\left(\mathcal{I}_{\xi}(F)\right) \rightarrow H^{0}\left(\mathcal{I}_{\xi^{\prime}}(F)\right) \rightarrow \mathbb{C}^{d-d^{\prime}} \rightarrow H^{1}\left(\mathcal{I}_{\xi}(F)\right) \rightarrow H^{1}\left(\mathcal{I}_{\xi^{\prime}}(F)\right) \rightarrow 0
$$

where $d^{\prime}$ is the degree of $\xi^{\prime}$, and consider the dimensions of the spaces. We know that because $\xi^{\prime} \subset \xi$, we have $d^{\prime}<d$ and $h^{0}\left(\mathcal{I}_{\xi^{\prime}}(F)\right)=h^{0}\left(\mathcal{I}_{\xi}(F)\right)=1$, and that $\operatorname{dim}$ $\mathbb{C}^{d-d^{\prime}}=d-d^{\prime}$, so an alternating sum argument tells us that $h^{1}\left(\mathcal{I}_{\xi^{\prime}}(F)\right)=d^{\prime}-1<$ $d-1=h^{1}\left(\mathcal{I}_{\xi}(F)\right)$.

This completes the proof of 4.2.0.10.

\subsection{The Correspondence Between $\mathbf{J}(S)$ and Vector Bundles}

\subsubsection{Theorem. $\mathbf{J}(S) \cong M_{H}(d, F, 0)$.}

We will prove the theorem by constructing a map and showing that it is an isomorphism. First we show that the target space is nonempty.

4.3.0.13 Theorem. $M_{H}(d, F, 0)$ is nonempty and compact.

In order to prove this, we need the following fact:

4.3.0.14 Fact $[\operatorname{Dolg} 4]$. There exists a $\mu$-stable $E$ with vector $(d, F, 0)$, constructed as in 4.2.0.10.

4.3.0.15 Proof of 4.3.0.13 A $\mu$-stable vector bundle $E$ is simple ([Mukai1]), and the dimension of $\operatorname{Ext}_{\mathcal{O}_{S}}^{1}(E, E)$ is 2 by [Tyurin, eqn. (4.2)]. Under these conditions, we may apply [Mukai2, Theorem 4.3] to see that there exists some ample $H$ such that $M_{H}(d, F, 0)$ is nonempty and compact. 
$\mathbf{J}(S)$ is defined to be the compactification of $\operatorname{Jac}\left(S_{\eta}\right) \cong \operatorname{Pic} c^{\circ}\left(S_{\eta}\right)$ (with the correspondence between the distinguished point created on $S_{\eta}$ and a distinguished divisor $\sum n_{i} p_{i}$ such that $\left.\sum n_{i}=0\right)$. Another way to view the Jacobian fibration is as a compactification of the relative Picard variety $\underline{\mathrm{Pic}}_{S / \mathbb{P}^{1}}^{\circ}[$ Enriques I].

Now examine the symmetric product $S^{(d)}$, where points are 0-cycles on $S$. If we restrict the fibration $f: S \rightarrow \mathbb{P}^{1}$ to the smooth fibres ( as $f^{\prime}: S^{\prime} \rightarrow U \subset \mathbb{P}^{1}$ ), we may consider the relative symmetric product $S_{\mathbb{P}^{1}}^{(d)}$; points of this variety are 0-cycles $\xi$ on $S$, contained in the generic fibre $S_{\eta}$. Note that the closure of such a 0 -cycle is a $d$-section (denoted $D)$.

If we take a map from $S_{\mathbb{P}^{1}}^{(d)}$ to $M_{H}(d, F, 0)$ by sending a 0 -cycle to a vector bundle, then this map will factor through $\underline{\mathrm{Pic}}_{S / \mathbb{P}^{1}}^{\circ}$ because $S$ has a $d$-section. Thus, the image of the space $S_{\mathbb{P}^{1}}^{(d)}$ is Zariski open in $\mathbf{J}(S)$.

\subsubsection{Definition.}

$$
\begin{aligned}
\alpha: \mathbf{J}(S) & \rightarrow M_{H}(d, F, 0) \\
\alpha(\xi) & \mapsto E(\xi, F)
\end{aligned}
$$

This map begins with $\xi \in S_{\mathbb{P}^{1}}^{(d)}$. (We consider its closure in $S$.) Then we use Theorem 4.2.0.10 to produce a vector bundle $E(\xi, F)$.

Of course, we need to know that $E(\xi, F) \in M_{H}(d, F, 0)$; this is a consequence of 4.3.0.14.

\subsubsection{Proof that $\alpha$ is well-defined}

Recall that the closure of $\xi$ in $S$ is a $d$-section $D$. If $D \sim D^{\prime} \in \operatorname{Pic}^{d}\left(S_{\eta}\right)$, then $\mathcal{O}_{S_{\eta}}(D) \cong \mathcal{O}_{S_{\eta}}\left(D^{\prime}\right)$. If we choose a rational function $f$ on $S_{\eta}$ such that $(f)_{0}=D$ and $(f)_{\infty}=D^{\prime}$, then this gives us an isomorphism $\mathcal{O}_{S_{\eta}}(D) \rightarrow \mathcal{O}_{S_{\eta}}\left(D^{\prime}\right)$ and when we 
restrict this to a suitable fibre $F$, we then have an isomorphism $\mathcal{O}_{F}(\xi) \rightarrow \mathcal{O}_{F}\left(\xi^{\prime}\right)$. Then we may apply [Tyurin K3, Lemma 2.5] which tells us that this implies that $E(\xi, F)=E\left(\xi^{\prime}, F\right)$. To choose $f$ suitably means that $f$ does not have any zeros or poles along $F$; this is always possible because there are an infinite number of fibres and $f$ can only have a finite number of zeros and poles.

4.3.1.1 Remark. Not every vector bundle $E$ has a cycle $\xi$ associated to it. For the many requirements $E$ must satisfy, see [Tyurin, $\S 2]$.

4.3.1.2 Definition. $B(E)$ is the variety of cycles associated to a bundle $E$.

\subsubsection{Proof that $\alpha$ is injective}

We need to show that if $E(\xi, F)=E\left(\xi^{\prime}, F\right)$ then $D \sim D^{\prime}$.

We can compute the dimension of $B(E)$; it is a Zariski open subset of the Grassmanian $G\left(r k(E)-1, H^{0}\left(E \otimes K_{S}^{*}\right)\right)$ [Tyurin, §3] which for us is $G\left(d-1, H^{0}(E)\right)=$ $\operatorname{Gr}(d-1, d)$. This has dimension $(d-1)(d-(d-1))=d-1$ so $\operatorname{dim} B(E) \leq$ $d-1$. On the other hand, if $\xi$ produces $E$, then $\xi^{\prime} \sim \xi$ will also produce $E$ (see [Tyurin K3, Lemma 2.5]). What is the dimension of the space of cycles equivalent to $\xi$ ? It is $h^{0}\left(\mathcal{O}_{F}(\xi)\right)$, which we can compute using Riemann-Roch for curves: $h^{0}\left(\mathcal{O}_{F}(\xi)\right)-h^{0}\left(\mathcal{O}_{F}(-\xi)\right)=d+1-g_{F} \cdot h^{0}\left(\mathcal{O}_{F}(-\xi)\right)=0$ as $\xi$ is effective, and $g_{F}=1$ as $F$ is elliptic. So $h^{0}\left(\mathcal{O}_{F}(\xi)\right)=d$ which corresponds to projective dimension $d-1$. This is the minimum dimension of $B(E)$ - after all, there could be some other cycle $\xi^{\prime} \nsim \xi$ which also produces $E$. But as $\operatorname{dim} B(E) \leq d-1$ and $\geq d-1$, it equals $d-1$ and so we have that if $E(\xi, F)=E\left(\xi^{\prime}, F\right)$ then $\xi \sim \xi^{\prime}$.

Now we must show that if $\xi \sim \xi^{\prime}$ then $D \sim D^{\prime}$. This is not hard: our results above hold for most $F$ (i.e. $F$ hasn't the wrong zeros and poles for $\xi$ ) so if $\xi \sim \xi^{\prime}$ then 
$\mathcal{O}_{F}(\xi)=\mathcal{O}_{F}\left(\xi^{\prime}\right)$ for general $F$, which is the same as saying $\mathcal{O}_{F}\left(\left.D\right|_{F}\right)=\mathcal{O}_{F}\left(\left.D^{\prime}\right|_{F}\right)$ for general $F$, where $D$ and $D^{\prime}$ are constructed by extending $\xi$ across the $F$. (Recall that we chose $\xi, \xi^{\prime}$ by intersecting a $d$-section with $F$.) So $\mathcal{O}_{S_{\eta}}\left(\left.D\right|_{F}\right)=\mathcal{O}_{S_{\eta}}\left(\left.D^{\prime}\right|_{F}\right)$ for generic $F$, so $\mathcal{O}_{S_{\eta}}(D)=\mathcal{O}_{S_{\eta}}\left(D^{\prime}\right)$ which means that $D \sim D^{\prime}$.

4.3.2.1 Note: There is an error in Tyurin's calculation of $\operatorname{dim} B(E)$; he claims that in our case $(s \leq 0)$ that $\operatorname{dim} B(E)=0$ [Tyurin, Lemma 4.1(5)], which we have shown it is not. For K3 surfaces, the only case where $E(\xi, F)$ has $\operatorname{dim} B(E)=0$ is when $r k(E)=d-1, c_{2}(E)=d$.

\subsubsection{Proof that $\alpha$ is an isomorphism of varieties}

One can show that $\alpha$ is algebraic. By [Mukai2, Theorem 1.4], $M_{H}(d, F, 0)$ is a minimal irreducible $\mathrm{K} 3$ surface. Because $\alpha$ is an injection between projective varieties of the same dimension, it must be a birational morphism; now, because $\mathbf{J}(S)$ and $M_{H}(d, F, 0)$ are both minimal, we satisfy the conditions of the minimal model theorem - thus, $\alpha$ is an isomorphism.

\subsection{The Map $\operatorname{Pic}(S) \hookrightarrow \operatorname{Pic}(\mathbf{J}(S))$}

4.4.0.1 Theorem. If $S$ has multisection index $d$, then $\operatorname{Pic}(S)$ embeds in $\operatorname{Pic}(\mathbf{J}(S))$ with index $d$.

We will prove this theorem by showing that we can use a theorem of Mukai to produce this map.

4.4.0.2 Theorem [Mukai2, Theorem 1.5 and Proposition 6.4]. Suppose that $v$ is primitive and isotropic, and that $M_{H}(v)$ is nonempty and compact. Then - There is an isometry $\phi: v^{\perp} / \mathbb{Z} v \rightarrow H^{2}\left(M_{H}(v), \mathbb{Z}\right)$ which induces a Hodge isometry 
on transcendental cycles $\phi^{\prime}: T_{S} \rightarrow T_{M_{H}(v)}$.

- $\operatorname{coker} \phi \cong \mathbb{Z}_{d}$, where $d=\min _{u}\left\{|\langle u \cdot v\rangle|, u \in \tilde{H}^{1,1}(S, \mathbb{Z}),\langle u \cdot v\rangle \neq 0\right\}$.

Recall that a Hodge isometry preserves cup product and Hodge structure, i.e. $\phi\left(H^{2,0}(S)\right)=H^{2,0}\left(M_{H}(v)\right)$. By 4.3.0.13, we have satisfied the hypotheses of 4.4.0.2; in this case, we have that $\mathbf{J}(S) \cong M_{H}(d, F, 0)$. It remains to show that $d$ above is the multisection index, and that the map $\phi$ is also a map on the Picard lattices.

4.4.0.3 Fact. $d$ in Theorem 4.4.0.2 is the same as $d$ our multisection index.

Proof of 4.4.0.3. $u \in \tilde{H}^{1,1}(S, \mathbb{Z})$ means that $u=(s, \mathcal{L}, t)$ where $s, t \in \mathbb{Z}$ and $\mathcal{L} \in \operatorname{Pic}(S)=H^{1,1}(S, \mathbb{Z}) .(u \cdot v)=\mathcal{L} \cdot[F]-d s-0 ; s$ can be any integer and $\mathcal{L} \cdot[F]$ can be either 0 (if $\mathcal{L}=\left[F^{\prime}\right]$ ) or $k d$ if $\mathcal{L}$ is horizontal. (Combinations of horizontal and vertical curves have $\mathcal{L} \cdot[F]=k^{\prime} d$ and reducible-fibre components have $\mathcal{L} \cdot[F]=0$.) In summary, $(u \cdot v)=(k-s) d$ where we allow either $k$ or $s$ to be 0 . So in other words, the range of values for $(u \cdot v)$ is multiples of $d$, and the minimal positive element in that set is $d$.

4.4.0.4 Fact. The map $\phi$ extends to a map $\phi^{\text {ext/res }}: \operatorname{Pic}(S) \rightarrow \operatorname{Pic}(\mathbf{J}(S))$.

Proof of 4.4.0.4. We know $\left(T_{S}\right)_{H^{2}}^{\perp}=\operatorname{Pic}(S)$. $\phi$ is a Hodge isometry, induced from a homomorphism of Hodge structures [Mukai2, paragraph before Thm 1.5] so in particular, there is a homomorphism $\phi^{e x t}$ of Hodge structures on $H^{2}$, which takes

$$
\begin{gathered}
H_{S}^{2,0} \rightarrow H_{\mathbf{J}(S)}^{2,0} \\
\operatorname{Pic}(S) \cong H_{S}^{1,1} \rightarrow H_{\mathbf{J}(S)}^{1,1} \cong \operatorname{Pic}(\mathbf{J}(S)) \\
H_{S}^{0,2} \rightarrow H_{\mathbf{J}(S)}^{0,2}
\end{gathered}
$$


Therefore, as this map preserves transcendental cycles $T_{S}$, and because $T_{S}$ embeds primitively into $H^{2}$, it preserves algebraic cycles as well $(\operatorname{Pic}(S))$. So we may restrict $\phi^{e x t}$ to a map $\phi^{e x t / r e s}$ on the Picard lattices.

Now we can calculate coker $\phi^{\text {ext/res }}$. coker $\phi=\mathbb{Z}_{d}$ and the discriminants of $T_{S}$ and $\operatorname{Pic}(S)$ are the same by definition (they are orthogonal complements, so $\left.q_{T_{S}}=-q_{\text {Pic }(S)}\right)$, so coker $\phi^{\text {ext } / \text { res }}=\mathbb{Z}_{d}$ as well.

Aside. Why does this make sense? We know that disc $\operatorname{Pic}(\mathbf{J}(S))$ divides disc $\operatorname{Pic}(S)$, and they could be equal. If the index of $\operatorname{Pic}(S)$ in $\operatorname{Pic}(\mathbf{J}(S))$ is $n$, then disc $\operatorname{Pic}(S) /$ disc $\operatorname{Pic}(\mathbf{J}(S))=n^{2}$ because $\operatorname{Pic}(S) \hookrightarrow_{n} \operatorname{Pic}(\mathbf{J}(S)) \hookrightarrow \operatorname{Pic}(\mathbf{J}(S))^{\star} \hookrightarrow_{n}$ $\operatorname{Pic}(S)^{\star}$, and $L \hookrightarrow_{n} M \hookrightarrow M^{\star} \hookrightarrow_{n} L^{\star}$ means $L^{\star} / L=M^{\star} / M \cdot n^{2}$. Therefore we expect $\operatorname{Pic}(\mathbf{J}(S)) / \phi^{e x t / r e s}(\operatorname{Pic}(S))$ to be $n(=d)$.

This completes our proof of Theorem 4.4.0.1.

4.4.0.5 Note. Mukai often claims that his results hold for $\rho>11$. Reading his papers carefully reveals that this is because he is only certain that $T_{S}$ embeds primitively into $H^{2}$ for $\rho>11$. However, for us $\operatorname{Pic}(S)$ always embeds primitively into $H^{2}$; primitive embeddings are defined for orthogonal pairs, so $T_{S}$ embeds primitively as well. Thus, we may use Mukai's results when $\rho \leq 11$.

\subsection{Further Conjectures}

4.5.0.6 Conjecture (Dolgachev). The discriminants $\operatorname{disc}(\operatorname{Pic}(S))$ and $\operatorname{disc}(\operatorname{Pic}(\mathbf{J}(S)))$ of an elliptic surface are related by the formula

$$
\operatorname{disc}(\operatorname{Pic}(S) / \text { torsion })=\left(\frac{\text { index }}{\operatorname{lcm}\left(m_{i}\right)}\right)^{2} \operatorname{disc}(\operatorname{Pic}(\mathbf{J}(S))),
$$


where the index is that of the lowest multisection and the $m_{i}$ are the multiplicities of multiple fibres.

A stronger conjecture, on which I am also working, is that there is a map $\operatorname{Pic}(S) \rightarrow$ $\operatorname{Pic}(\mathbf{J}(S))$ so that

$$
[\operatorname{Pic}(\mathbf{J}(S)): \operatorname{Pic}(S) / \text { torsion }]=\frac{\text { index }}{\operatorname{lcm}\left(m_{i}\right)}
$$




\section{APPENDICES}




\section{APPENDIX A}

\section{Tables of Forms and Values}

We list the forms and their values for every form used in this thesis. After these tables, we list the possible forms for popular discriminants such as 16, 27, and 32 .

Table A.1: Forms and Values for $p \neq 2$

\begin{tabular}{|c|c|c|c|}
\hline Form & $\bmod 2 \mathbb{Z}$ & $\bmod \mathbb{Z}$ & $\operatorname{Diagram}(\mathrm{s})$ \\
\hline$w_{3,1}^{1}$ & $4 / 3$ & $2 / 3=-1 / 3$ & $A_{2}$ \\
\hline$w_{3,1}^{-1}$ & $2 / 3$ & $1 / 3=-2 / 3$ & $E_{6}$ \\
\hline$w_{3,2}^{1}$ & $4 / 9,16 / 9,0,10 / 9$ & $2 / 9=-7 / 9$ & $A_{8}$ \\
\hline$w_{3,2}^{-1}$ & $2 / 9,8 / 9,0,14 / 9$ & $1 / 9=-8 / 9$ & \\
\hline$w_{5,1}^{1}$ & $4 / 5,6 / 5$ & $2 / 5=-3 / 5$ & $A_{4}$ \\
\hline$w_{5,1}^{-1}$ & $2 / 5,8 / 5$ & $1 / 5=-4 / 5$ & $T_{2,5,5}$ \\
\hline$w_{7,1}^{1}$ & $2 / 7,8 / 7,4 / 7$ & $1 / 7=-6 / 7$ & $A_{6}$ \\
\hline$w_{7,1}^{-1}$ & $6 / 7,10 / 7,3 / 7$ & $3 / 7=-4 / 7$ & \\
\hline$w_{11,1}^{1}$ & $4 / 11,16 / 11,14 / 11,20 / 11$ & $2 / 11$ & $A_{10}$ \\
\hline$w_{11,1}^{-1}$ & $2 / 11,8 / 11,18 / 11,10 / 11$ & $1 / 11$ & \\
\hline $\begin{array}{l}w_{13,1}^{1} \\
w_{131}^{-1}\end{array}$ & $\begin{array}{l}4 / 13 \\
2 / 13\end{array}$ & & $T_{34.5}$ \\
\hline$w_{17,1}^{1}$ & $2 / 17$ & $1 / 17=-16 / 17$ & $A_{16}$ \\
\hline$w_{17,1}^{-1}$ & $6 / 17$ & $\begin{array}{l}3 / 17,12 / 17,10 / 17,14 / 17 \\
7 / 17,6 / 17,11 / 17,5 / 17\end{array}$ & \\
\hline$w_{19,1}^{1}$ & $4 / 19$ & $\begin{array}{l}2 / 19=-17 / 19 ; 8 / 19,18 / 19 \\
13 / 19,12 / 19,15 / 19,3 / 19 \\
14 / 19,10 / 19=-9 / 19\end{array}$ & $A_{18}$ \\
\hline \multicolumn{4}{|c|}{ continued on next page } \\
\hline
\end{tabular}




\begin{tabular}{|c|c|c|c|}
\hline \multicolumn{4}{|c|}{ continued from previous page } \\
\hline Form & $\bmod 2 \mathbb{Z}$ & $\bmod \mathbb{Z}$ & $\operatorname{Diagram}(\mathrm{s})$ \\
\hline$w_{19,1}^{-1}$ & $2 / 19$ & & \\
\hline $\mathbb{Z}_{10}$ & & $-9 / 20$ & $A_{9}$ \\
\hline $\mathbb{Z}_{14}$ & & $-13 / 28$ & $A_{13}$ \\
\hline $\mathbb{Z}_{12}$ & & $-11 / 24$ & $A_{11}$ \\
\hline $\mathbb{Z}_{16}$ & & 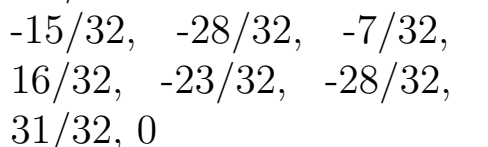 & $A_{15}$ \\
\hline $\mathbb{Z}_{18}$ & & $-17 / 36$ & $A_{17}$ \\
\hline
\end{tabular}

Table A.2: Forms and Values for $p=2$

\begin{tabular}{|c|l|l|c|}
\hline Form & \multicolumn{1}{|c|}{$\bmod 2 \mathbb{Z}$} & \multicolumn{1}{|c|}{$\bmod \mathbb{Z}$} & Diagram(s) \\
\hline$w_{2,1}^{1}$ & $1 / 2$ & $1 / 4=-3 / 4$ & $E_{7}$ \\
$w_{2,1}^{-1}$ & $-1 / 2=3 / 2$ & $3 / 4=-1 / 4$ & $A_{1}$ \\
$\left(w_{2,1}^{1}\right)^{2}$ & & & $D_{6} \equiv D_{14}$ \\
$\left(w_{2,1}^{-1}\right)^{2}$ & & & $D_{10}$ \\
$u$ & $0,0,1,0$ & $0,0,1 / 2,0$ & $D_{8}$ \\
$v$ & $1,1,1,0$ & $1 / 2,1 / 2,1 / 2,0$ & $D_{4} \equiv D_{12}$ \\
$w_{2,2}^{1}$ & $1 / 4,1$ & $-1 / 8$ & $D_{7}$ \\
$w_{2,2}^{-1}$ & $-1 / 4=7 / 4$ & $5 / 8=-3 / 8$ & $D_{9}$ \\
$w_{2,2}^{5}$ & $5 / 4,1$ & $-5 / 8$ & $A_{3}$ \\
$w_{2,2}^{-5}$ & $-5 / 4=3 / 4$ & $-7 / 16$ & $D_{5} \equiv D_{13}$ \\
$w_{2,3}^{1}$ & $1 / 8,1 / 2,9 / 8,0$ & & $A_{7}$ \\
$w_{2,3}^{-1}$ & $-1 / 8=15 / 8,3 / 2,7 / 8,0$ & & \\
$w_{2,3}^{5}$ & $5 / 8,1 / 2,13 / 8,0$ & & \\
$w_{2,3}^{-5}$ & $-5 / 8=11 / 8,3 / 2,3 / 8,0$ & & \\
$u_{2}$ & $0,0,0,0,0,1 / 2,1$, & $0,0,0,0,0,1 / 4,1 / 2$, & \\
& $3 / 2,0,1,0,1,0,3 / 2$, & $3 / 4,0,1 / 2,0,1 / 2,0$, & \\
$v_{2}$ & $1,1 / 2$ & $3 / 4,1 / 2,1 / 4$ & \\
& $0,1 / 2,0,1 / 2,1 / 2$, & $0,1 / 4,0,1 / 4,1 / 4$, & \\
& $3 / 2,1 / 2,1 / 2,3 / 2,3 / 2$ & $0,3 / 4,1 / 4,1 / 4,3 / 4$, & \\
\hline \hline
\end{tabular}

We also have the lattice $U$ which is of rank 2 and has trivial quadratic form.

A.0.0.7 Definition. $U(m)$ is a lattice of rank 2 which is defined on $\mathbb{Z}_{m} \times \mathbb{Z}_{m}$. It has quadratic form values $\frac{a b}{m}, a \in \mathbb{Z}_{m}, b \in \mathbb{Z}_{m}$, all $\bmod \mathbb{Z}$. 


\begin{tabular}{|l|l|l|}
\hline$U(m)$ & values & Brieskorn form \\
\hline$U(2)$ & $0,0,0,1 / 2$ & $u$ \\
\hline$U(3)$ & $0,0,0,0,1 / 3,2 / 3,0,2 / 3,1 / 3$ & $w_{3,1}^{1} \perp w_{3,1}^{-1}$ \\
\hline$U(4)$ & $0,0,0,0,0,1 / 4,1 / 2,3 / 4,0,1 / 2,0,1 / 2,0,3 / 4,1 / 2,1 / 4$ & $u_{2}$ \\
\hline
\end{tabular}

Table A.3: Values of the forms $U(m)$

\section{A.0.1 Discriminant group $\left(\mathbb{Z}_{2}\right)^{3}$}

There are only four possible forms.

$$
\begin{aligned}
& \left(w_{2,1}^{1}\right)^{3} \cong w_{2,1}^{-1} \perp v \\
& \left(w_{2,1}^{1}\right)^{2} \perp w_{2,1}^{-1} \cong w_{2,1}^{1} \perp u \\
& w_{2,1}^{1} \perp\left(w_{2,1}^{-1}\right)^{2} \cong w_{2,1}^{-1} \perp u \\
& \left(w_{2,1}^{-1}\right)^{3} \cong w_{2,1}^{1} \perp v
\end{aligned}
$$

\section{A.0.2 Discriminant group $\left(\mathbb{Z}_{2}\right)^{4}$}

There are only six possible forms.

$$
\begin{aligned}
& \left(w_{2,1}^{1}\right)^{4} \cong w_{2,1}^{1} \perp w_{2,1}^{-1} \perp v \cong\left(w_{2,1}^{-1}\right)^{4} \\
& \left(w_{2,1}^{1}\right)^{3} \perp w_{2,1}^{-1} \cong\left(w_{2,1}^{1}\right)^{2} \perp u \cong\left(w_{2,1}^{-1}\right)^{2} \perp v \\
& \left(w_{2,1}^{1}\right)^{2} \perp\left(w_{2,1}^{-1}\right)^{2} \cong w_{2,1}^{1} \perp w_{2,1}^{-1} \perp u \\
& \left(w_{2,1}^{-1}\right)^{3} \perp w_{2,1}^{1} \cong\left(w_{2,1}^{1}\right)^{2} \perp v \cong\left(w_{2,1}^{-1}\right)^{2} \perp u \\
& u \perp v \\
& v \perp v \cong u \perp u
\end{aligned}
$$

\section{A.0.3 Discriminant group $\left(\mathbb{Z}_{2}\right)^{5}$}

There are only four possible forms.

$$
\left(w_{2,1}^{1}\right)^{5} \cong\left(w_{2,1}^{1}\right)^{2} \perp w_{2,1}^{-1} \perp v \cong w_{2,1}^{1} \perp u \perp v \cong\left(w_{2,1}^{-1}\right)^{3} \perp u \cong\left(w_{2,1}^{-1}\right)^{4} \perp w_{2,1}^{1}
$$




$$
\begin{aligned}
& \left(w_{2,1}^{1}\right)^{4} \perp w_{2,1}^{-1} \cong w_{2,1}^{1} \perp\left(w_{2,1}^{-1}\right)^{2} \perp v \cong w_{2,1}^{-1} \perp u \perp v \cong\left(w_{2,1}^{1}\right)^{3} \perp u \cong\left(w_{2,1}^{-1}\right)^{5} \\
& \left(w_{2,1}^{1}\right)^{3} \perp\left(w_{2,1}^{-1}\right)^{2} \cong\left(w_{2,1}^{-1}\right)^{3} \perp v \cong w_{2,1}^{1} \perp v \perp v \cong w_{2,1}^{1} \perp u \perp u \cong\left(w_{2,1}^{1}\right)^{2} \perp w_{2,1}^{-1} \perp u \\
& \left(w_{2,1}^{1}\right)^{2} \perp\left(w_{2,1}^{-1}\right)^{3} \cong\left(w_{2,1}^{1}\right)^{3} \perp v \cong w_{2,1}^{-1} \perp v \perp v \cong w_{2,1}^{-1} \perp u \perp u \cong w_{2,1}^{1} \perp\left(w_{2,1}^{-1}\right)^{2} \perp u
\end{aligned}
$$

\section{A.0.4 Discriminant group $\left(\mathbb{Z}_{2}\right)^{6}$}

We will only analyze those with $u$ and $v$ as components. Then, we can have $u \perp u \perp u \cong u \perp v \perp v$ or $v \perp v \perp v \cong v \perp u \perp u$. We count the number of elements in $\left(\mathbb{Z}_{2}\right)^{6}$ with value 0 for each form; $u \perp u \perp u$ has 36 0-valued elements and $v \perp v \perp v$ has 280 -valued elements. This is an easy way to distinguish them.

\section{A.0.5 Discriminant group $\left(\mathbb{Z}_{3}\right)^{2}$}

There are only two possible forms. Table A.4 shows their values.

\begin{tabular}{|l|c|c|}
\hline elt. & $w_{3,1}^{1} \perp w_{3,1}^{-1}$ & $\left(w_{3,1}^{1}\right)^{2}$ \\
\hline$(0,0)$ & 0 & 0 \\
\hline$(0,1)$ & $2 / 3$ & $2 / 3$ \\
\hline$(0,2)$ & $2 / 3$ & $2 / 3$ \\
\hline$(1,0)$ & $4 / 3$ & $2 / 3$ \\
\hline$(1,1)$ & 0 & $4 / 3$ \\
\hline$(1,2)$ & 0 & $4 / 3$ \\
\hline$(2,0)$ & $4 / 3$ & $2 / 3$ \\
\hline$(2,1)$ & 0 & $4 / 3$ \\
\hline$(2,2)$ & 0 & $4 / 3$ \\
\hline
\end{tabular}

Table A.4: Values of the forms on $\left(\mathbb{Z}_{3}\right)^{2}$ 


\section{A.0.6 Discriminant group $\left(\mathbb{Z}_{3}\right)^{3}$}

There are only two possible forms.

$\left(w_{3,1}^{1}\right)^{3} \cong\left(w_{3,1}^{-1}\right)^{2} \perp w_{3,1}^{1}$ has 6 elements with value $4 / 3,12$ elements with value $2 / 3$, and 9 elements with value 0 .

$\left(w_{3,1}^{-1}\right)^{3} \cong\left(w_{3,1}^{1}\right)^{2} \perp w_{3,1}^{-1}$ has 6 elements with value $2 / 3,12$ elements with value $4 / 3$, and 9 elements with value 0 .

\section{A.0.7 Discriminant group $\left(\mathbb{Z}_{3}\right)^{4}$}

There are only two possible forms. Table A.5 shows their values.

$$
\begin{aligned}
& \left(w_{3,1}^{1}\right)^{4} \cong\left(w_{3,1}^{-1}\right)^{2} \perp\left(w_{3,1}^{1}\right)^{2} \cong\left(w_{3,1}^{-1}\right)^{4} \\
& \left(w_{3,1}^{1}\right)^{3} \perp w_{3,1}^{-1} \cong\left(w_{3,1}^{-1}\right)^{3} \cong w_{3,1}^{1}
\end{aligned}
$$

\begin{tabular}{|c|c|c|c|}
\hline elt. type & $\left(w_{3,1}^{1}\right)^{4}$ & $w_{3,1}^{1} \perp\left(w_{3,1}^{1}\right)^{3}$ & no. such elts. \\
\hline$(0,0,0,0)$ & 0 & 0 & 1 \\
\hline$(0,0,0,1)$ & $2 / 3$ & $2 / 3$ & 6 \\
\hline$(0,0,1,1)$ & $4 / 3$ & $4 / 3$ & 12 \\
\hline$(0,1,1,1)$ & 0 & 0 & 8 \\
\hline$(1,1,1,1)$ & $2 / 3$ & 0 & 16 \\
\hline$(1,0,0,0)$ & $2 / 3$ & $4 / 3$ & 2 \\
\hline$(1,0,0,1)$ & $4 / 3$ & 0 & 12 \\
\hline$(1,0,1,1)$ & 0 & $2 / 3$ & 24 \\
\hline
\end{tabular}

Table A.5: Values of the forms on $\left(\mathbb{Z}_{3}\right)^{4}$ 


\section{APPENDIX B}

\section{Yonemura.ma}

This appendix is an edited version of my Mathematica notebook Yonemura.ma.

Herein we document programs which, given the weight-vectors from Yonemura's paper,

- generate the rational polytope

- transform the rational polytope into 3-space

- find the extremal points of the Newton Polytope

- find the extremal points of the dual of each Newton Polytope

- give the f-vector and face-lattice for each Newton Polytope and its dual.

Please note that these procedures will not work for objects other than hypersurfaces in weighted projective space, with the exception of the functions which determine the f-vector and face-lattice.

Given a weight-vector $\left\{w_{1}, w_{2}, w_{3}, w_{4}\right\}$ and the total weight $\left.w=w_{1}+w_{2}+w_{3}+w_{4}\right)$, we can obtain the rational polytope associated to this weight-vector; it is defined as the portion of the hyperplane $w_{1} x_{1}+w_{2} x_{2}+w_{3} x_{3}+w_{4} x_{4}=w$ where $x_{i} \geq 0$. It is also the convex hull of the points $\left\{w / w_{1}, 0,0,0\right\},\left\{0, w / w_{2}, 0,0\right\},\left\{0,0, w / w_{3}, 0\right\},\left\{0,0,0, w / w_{4}\right\}$.

The Newton Polytope of this rational polytope is the convex hull of the integer points in the rational polytope. We list all integer points in a rectangular box 
enclosing the appropriate sector of the hyperplane, and check each one to see if it is in the rational polytope. We will use this list of integer points to generate the extremal points of the Newton Polytope. In the following cell, BoxofPoints generates all integer points in the rectangular box, and ListIntegerPoints tells us which of those are in the rational polytope.

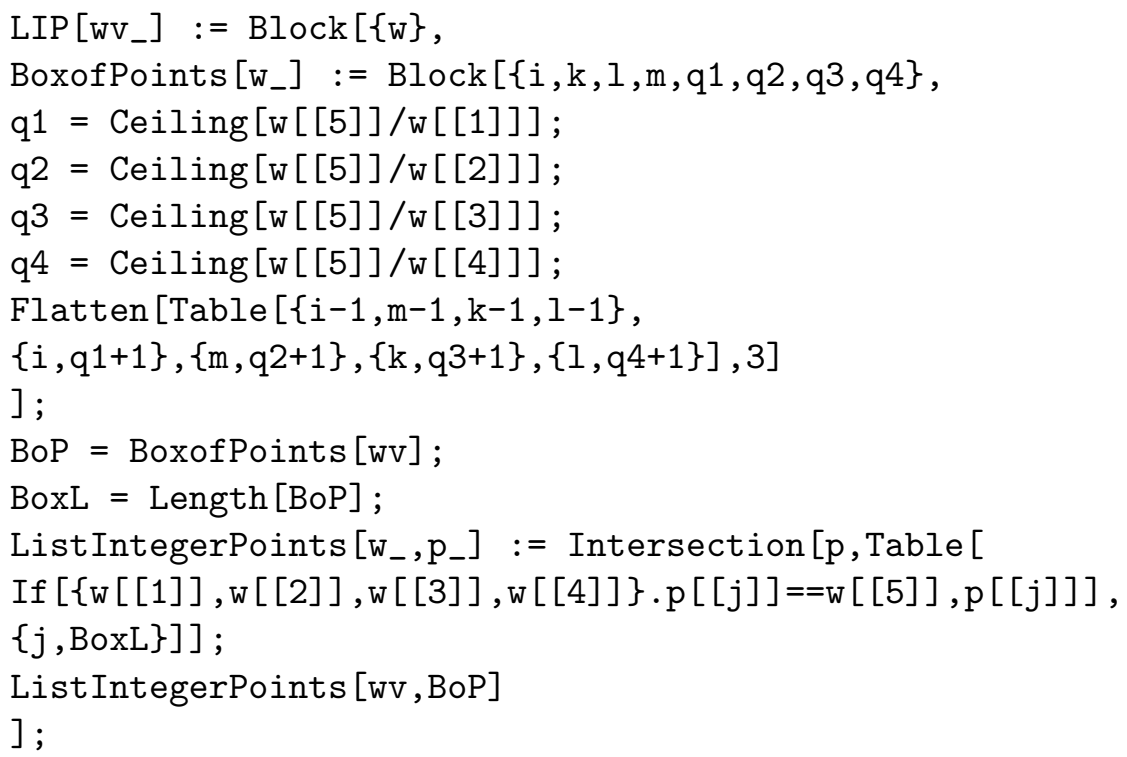

One would think that the easiest thing to do would be to use the VertexEnumeration $[\mathbf{V E}]$ procedure to get the extremal points of the Newton Polytope, by taking the convex hull of our LIP.

As it turns out, it is simpler to find each dual $\left(P^{*}\right)$, and then dualize $P^{*}$. Note that the dimension of our dual depends on our ambient space. If we take the dual of a polygon contained in $\mathbb{R}^{3}$, for example, we get an infinite cylinder; therefore, we must translate our LIP into $\mathbb{R}^{3}$ by transforming the lattice it lives in to $\mathbb{Z}^{3}$.

We use an algorithm given by Lev Borisov to get a 4 by 3 transformation matrix from the lattice in our hyperplane to $\mathbb{Z}^{3}$. Unfortunately, this map is not surjective.

TransMat [wv_] := Block $[\{\mathrm{q} 1, \mathrm{q} 2, \mathrm{q} 3, \mathrm{q} 4, \mathrm{r}, \mathrm{s}, \mathrm{t}, \mathrm{k}, \mathrm{l}, \mathrm{u} 1, \mathrm{u} 2, \mathrm{TM}\}$, $\mathrm{q} 1=w \mathrm{w}[[1]] ; \mathrm{q} 2=\mathrm{wv}[[2]] ; \mathrm{q} 3=\mathrm{wv}[[3]] ; \mathrm{q} 4=\mathrm{wv}[[4]] ;$ $\mathrm{x}[4]=r * \mathrm{GCD}[\mathrm{q} 1, \mathrm{q} 2, \mathrm{q} 3] / \mathrm{GCD}[\mathrm{q} 1, \mathrm{q} 2, \mathrm{q} 3, \mathrm{q} 4]$; 


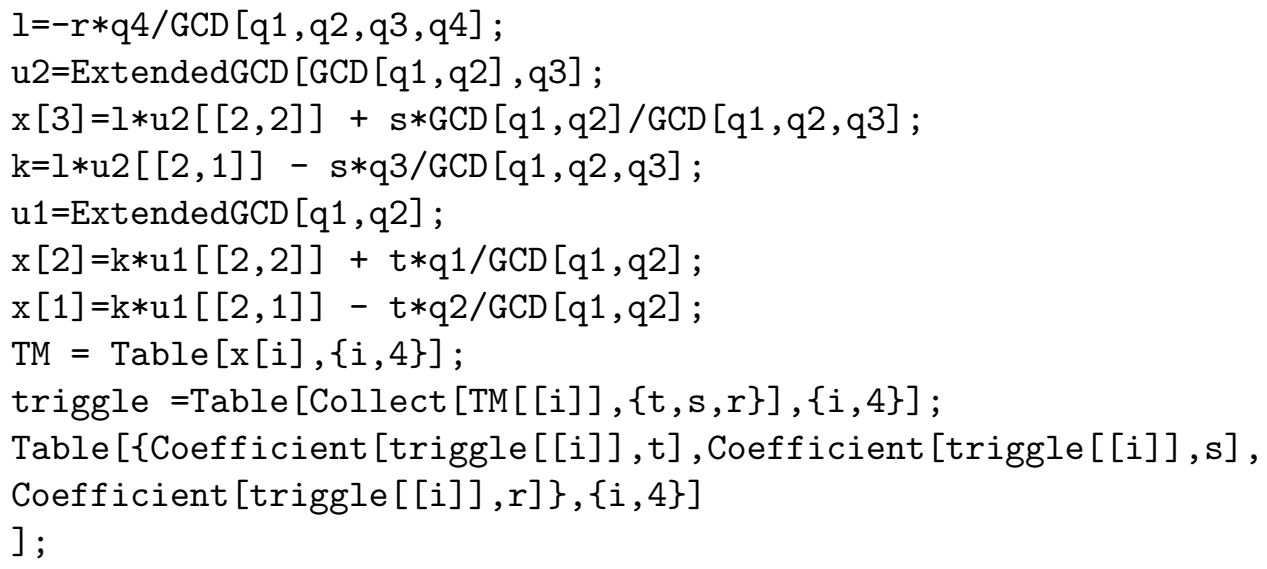

Notice that the point $(1,1,1,1)$ is in each hyperplane because $\sum\left(w_{i}\right)=w$. Thus, we translate the hyperplane to the origin by subtracting this vector. Then we have to take care of the fact that our map isn't surjective. We know that the columns of the matrix supply us with three vectors $\left(v_{1}, v_{2}, v_{3}\right)$ such that each point in our hyperplane can be expressed as a unique combination of them: $p=a_{1} v_{1}+a_{2} v_{2}+a_{3} v_{3}$ for scalars $a_{i}$. This equation gives us our point in 3 -space $\left(a_{1}, a_{2}, a_{3}\right)$. We add the point $p$ as the fourth column of the transformation matrix, and find the vector which generates the null space of the resulting matrix; this is the point $\vec{a}$. (This technique provided by Prof. Dolgachev.)

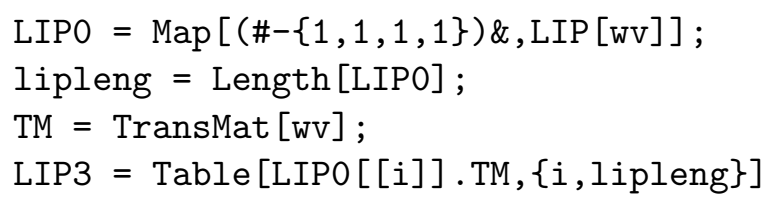

As it turns out, while VertexEnumeration is extremely accurate, it's too slow given the huge number of points we're dealing with in some cases. Instead we send the data through qhull, a program which takes the hull less accurately but much faster. The following program makes the appropriate 95 input files for qhull.

WilliamMatrix $\left[1_{-}, w_{-}\right]:=$Table $[\{1[[i, 1]], 1[[i, 2]]$, $I[[i, 3]], w v[i]]\},\{i, 4\}]$ 


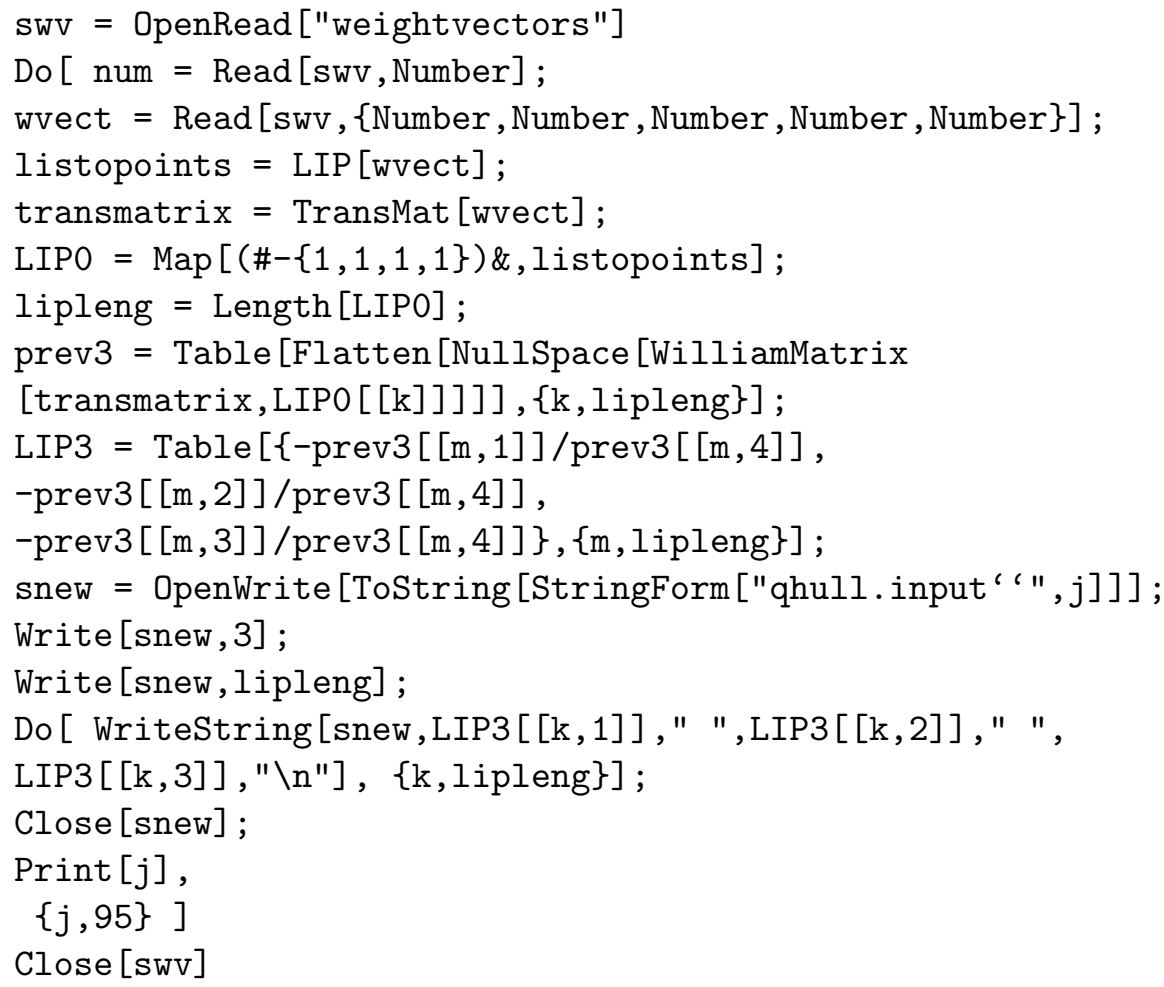

Now we have output from qhull (extP3). Are these polytopes reflexive? Luckily, one of the definitions of reflexivity for an integral polytope is that its dual is integral. So by computing all the duals, we can answer this question. By outputting the fvectors and face-lattices of each polytope (and its dual), we can begin to determine if any are isomorphic to each other (none are).

Recall that there is an easy way of finding the dual of a polytope, from [Brøndsted, Theorem 9.1]. If $P=\operatorname{conv}\left\{p_{i}\right\}$, then $P^{*}=\cap_{i}\left[K\left(p_{i}, 1\right)\right]$, where the $K\left(p_{i}, 1\right)$ are closed halfspaces with $p_{i}$ as the outward normal vectors, and the equation of the bounding hyperplanes are set equal to 1.

This should find the dual of a polytope given by extP3 [wv]. Notice that the origin is automatically contained in this set, as we subtracted $(1,1,1,1)$ already and the transformation matrix takes $(0,0,0,0)$ to $(0,0,0)$. The code does this: Load in the package, then shift the whole mess of hyperplanes about 1000 points away, 
so that we are assured that the whole dual will be contained in the positive- only quadrant (the DualM.c lines). We find the vertices of the resulting polytope, and then translate them back to the origin.

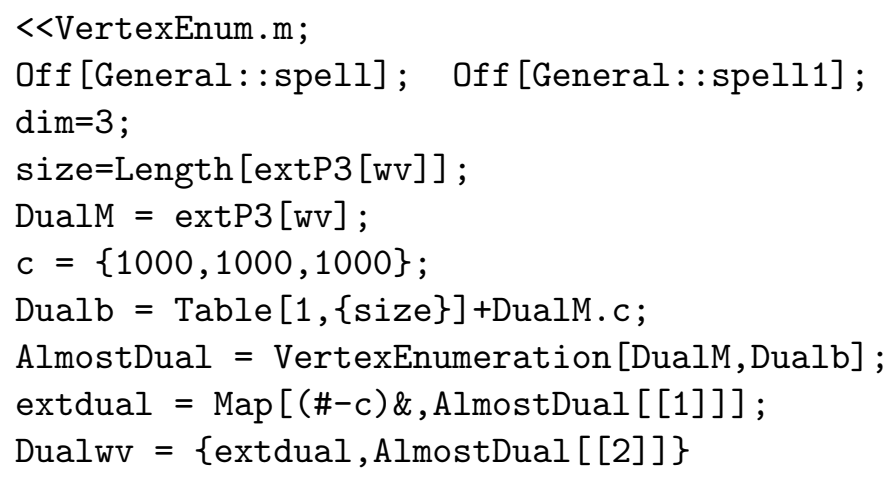

To find the extremal points etc. of the original polytope, we do almost exactly the same thing.

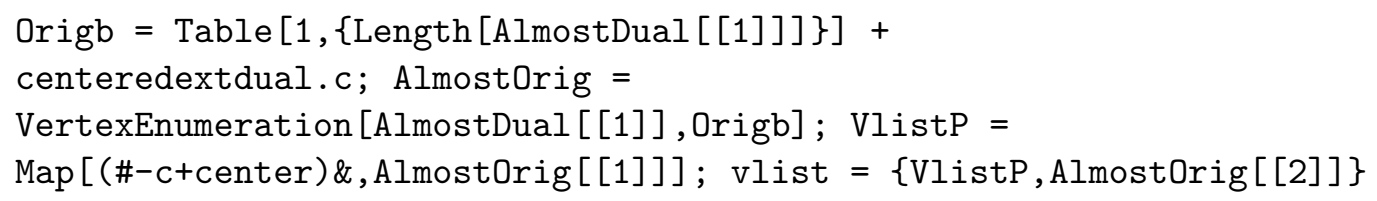

We also need to output the f-vector and a diagram of the face-lattice for each polytope and its dual. Once we have specified our polytope as vlist, then the FaceLattice package operates on its activesets (sets of hyperplane conditions each vertex satisfies) to produce the f-vector and the face-lattice. To do the same thing for the dual, we use the same code but with Dualwv and Dflist. You'll notice an interesting extra command, flinks. This outputs a string where "each element $\mathrm{k}, \mathrm{m}, \mathrm{k}+1, \mathrm{n} .$. , indicates that the $\mathrm{m}$-th face of dimension $\mathrm{k}$ is covered by the $\mathrm{n}$-th face of dimension $\mathrm{k}+1 . "$ (from documentation with $[\mathbf{F L}]$ )

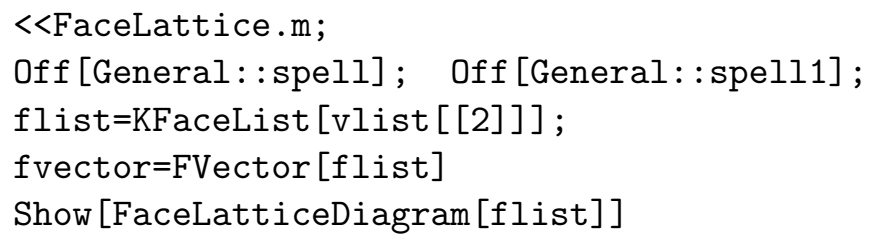




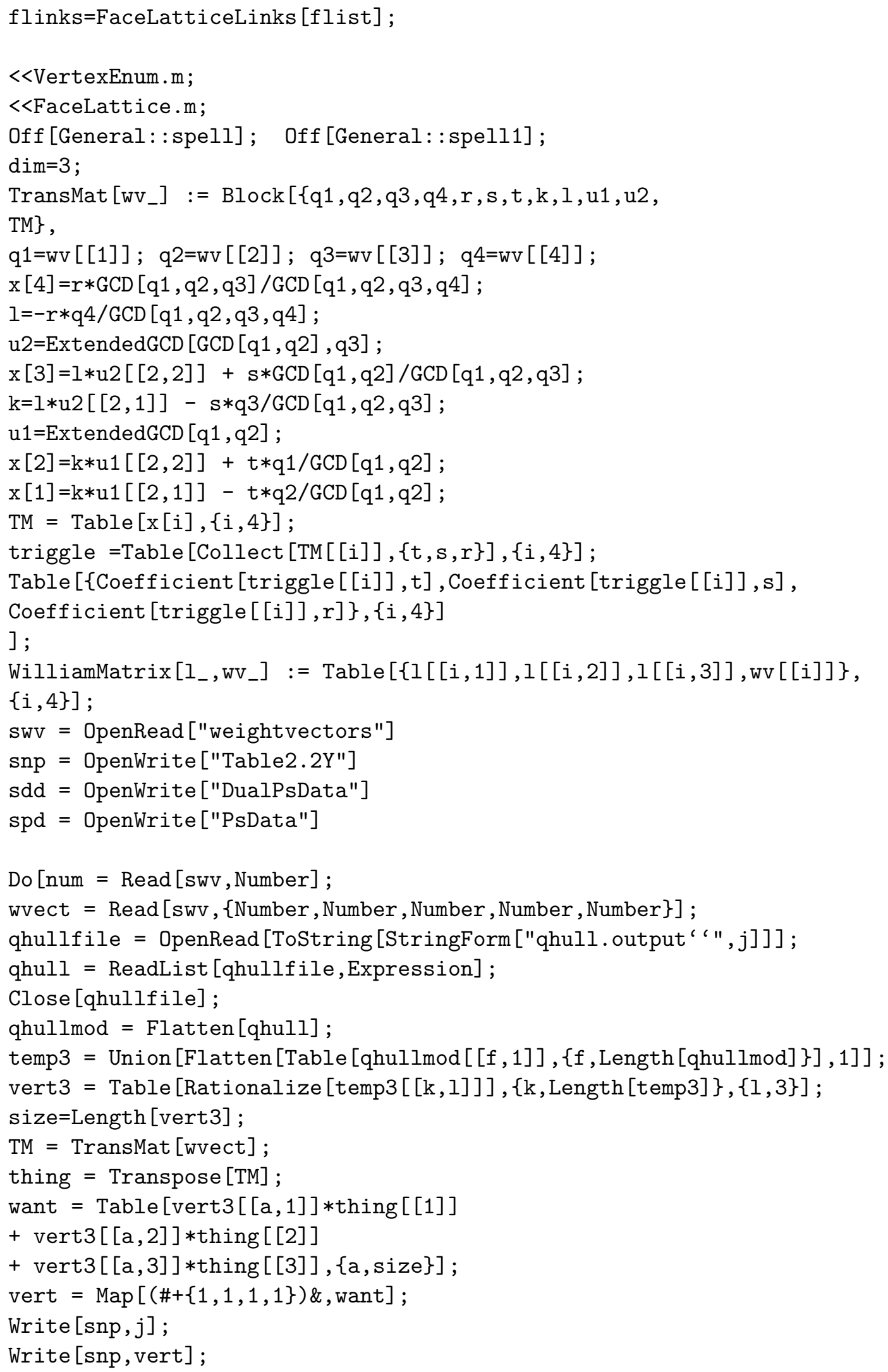




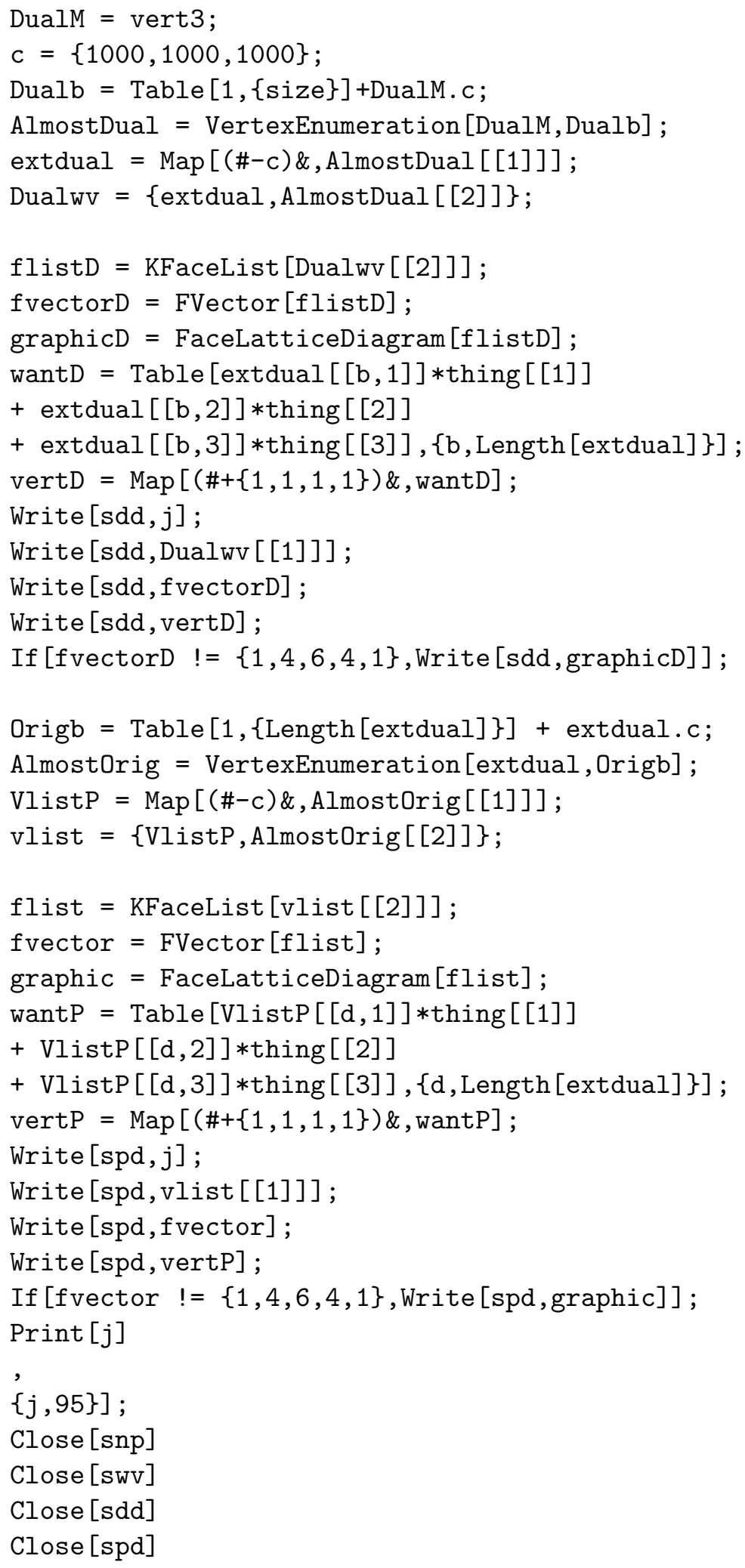




\section{APPENDIX C}

\section{Desingularize.ma}

This appendix is an edited version of my Mathematica notebook Desingularize.ma. Herein we document programs which compute canonical classes of 2-dimensional toric varieties and desingularize them.

\section{CanonicalClass [dualverts_]}

The input is a list of vertices for the polar dual polyhedron, and the output is a formal sum of codimension 1 subvarieties (in this case they are faces or subsets thereof, and so are denoted $\mathrm{F}[\mathrm{i}])$. If one begins with any of the usual descriptions of the polyhedron D corresponding to PD, it is simple to use the VertexEnumeration package $[\mathbf{V E}]$ to derive the vertices of the polar dual.

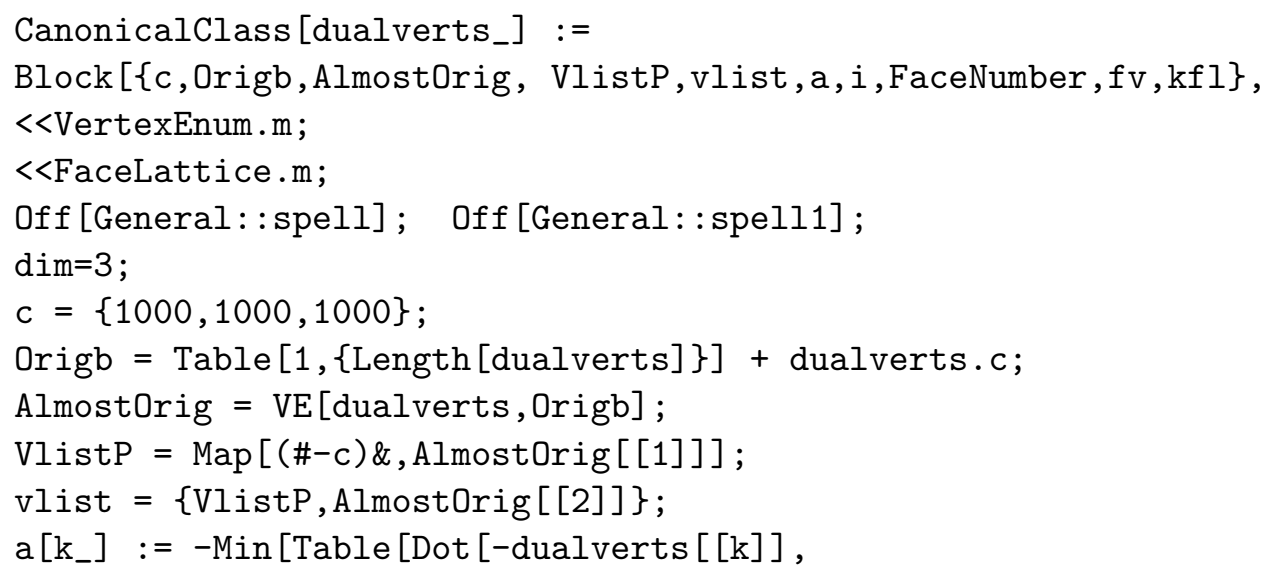




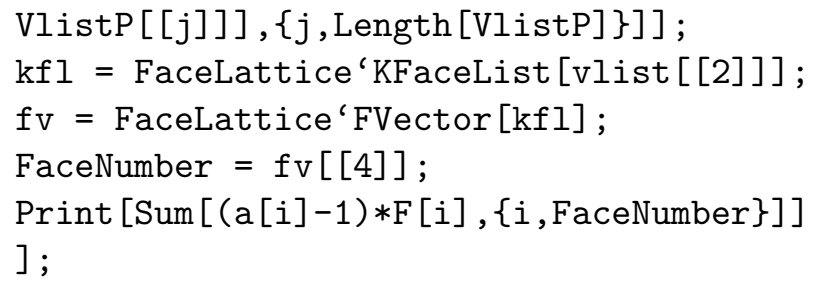

Explanation of the code: First we load in the packages required. We move the dual polytope far into the all-positive orthant so that VertexEnumeration will work correctly. The dual's vertices also correspond to positive normal vectors to the bounding hyperplanes of the polytope, and VE uses this information to produce information on the original polytope. We use this information to compute with the formula $K_{X}=\mathcal{O}\left(S\left(a_{i}-1\right) * F_{i}\right)$, where the $a_{i}$ are defined by $a_{i}=\min _{j}<n_{i}, v_{j}>$, the $n_{i}$ are the negative normal vectors to the faces, and the $v_{j}$ are the vertices.

SingGraph [dualverts_, LIP_, filename_] .

This command, given the vertices for the dual polytope (positive normal vectors to the faces of the polytope) and a filename, writes a description of the graph obtained by desingularizing the polytope. It also puts out a file filename.extras, which notes which edges of the graph are multiple and what genus the curve corresponding to each face has. Note: This command is designed to be executed on only one polytope! There is another command which is designed to work within a loop (not detailed in this appendix). One can view the results of SingGraph by loading the Combinatorica package and using the command

ShowLabeledGraph [ReadGraph ["filename"] ]. I chose to write the specifications of the graph to a file to minimize possible memory problems for Mathematica in storing both the huge matrix for a graph and the graphics primitives.

SingGraph [dualverts_, LIP_, filename_] := Block $[\{c$, Origb, 


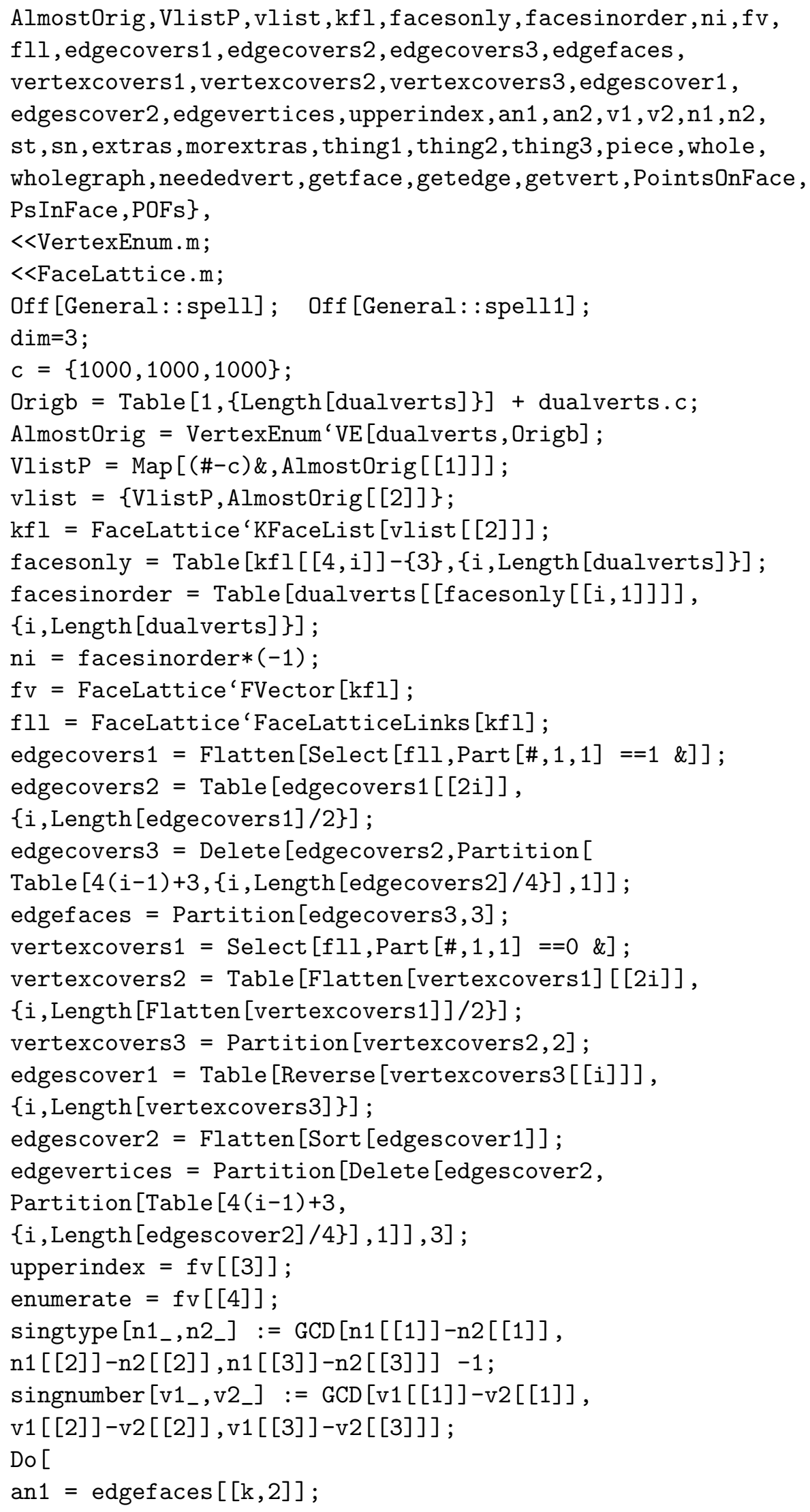




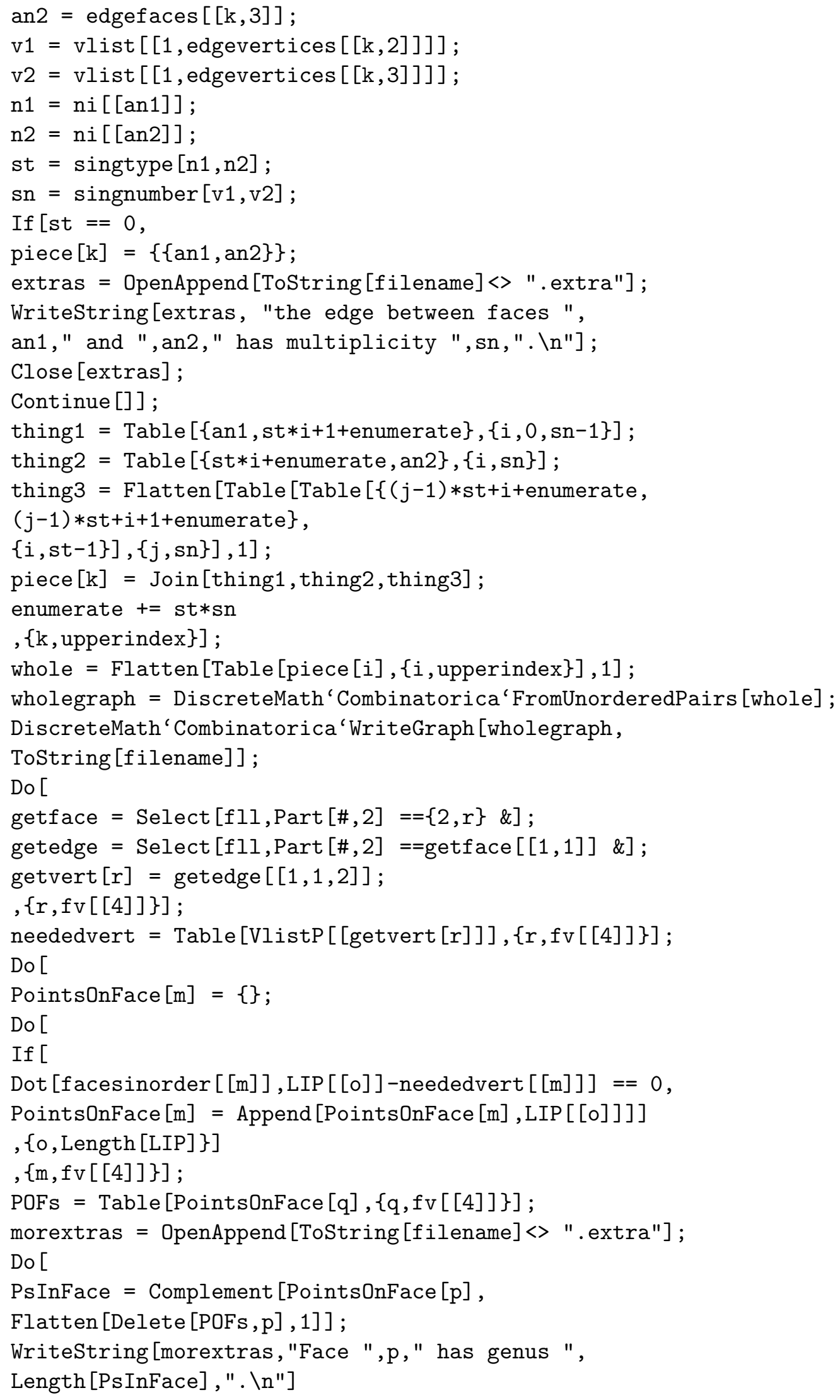


$,\{p, f v[[4]]\}]$;

Close [morextras]

];

The few lines of code (through the definition of the list ni) serve the purpose of correlating the enumeration of our list of the faces to the enumeration of the VertexEnumeration and KFaceList process. After that, reformat the cover relations for vertices and edges from the face-lattice poset. This format is lists (edgefaces

and edgevertices) $\{\{$ edge, face 1, face 2$\}, \ldots\}$ and $\{\{$ edge, vertex 1, vertex 2$\}, \ldots\}$ respectively, corresponding to the fact that each edge is the intersection of two faces and each edge has a vertex on each end.

Now we define two functions (singtype and singnumber) which tell us the type and multiplicity of singularity for each edge. Even if edge is nonsingular, it may have multiplicity anyway; so, we write this information to the file filename.extras.

For each edge, we determine the number and type of singularity, and create a list of unordered pairs which tell us exactly where edges should be in the graph. (For example, a pair 7,3 tells us that vertex 7 is joined to vertex 3 by an edge.) Finally, we put all the information from the edges into one big list, being careful with the labeling, and create and write to a file the Mathematica information for the graph.

The remaining code determines the number of points in the interior of each face, i.e. the genus of the corresponding curve. To do this, we dot LIP with the normal vector to the plane of the face. Any vector calculus book will tell you that this only works if we first subtract off some point in that plane, so the getstuff variables perform the function of finding some vertex on the face. After we know which points are on each face, we check to see which points are on no edge. These are in the interior of the face. Then, of course, we write all this to filename.extras. 


\section{APPENDIX D}

\section{$M_{\vec{p}, \vec{i}, k}$ programs}

Here is program which computes $\vec{i}$ and the discriminant for one of the hypersurfaces. The inputs are the weight-vector, degree, $\vec{p}$ and $k$. The program generates all possible $\vec{i}$ and tests each in the formula for $h^{0}\left(S, O_{S}(K)\right)$; if the result is an integer, the procedure prints that $\vec{i}$ and the associated discriminant.

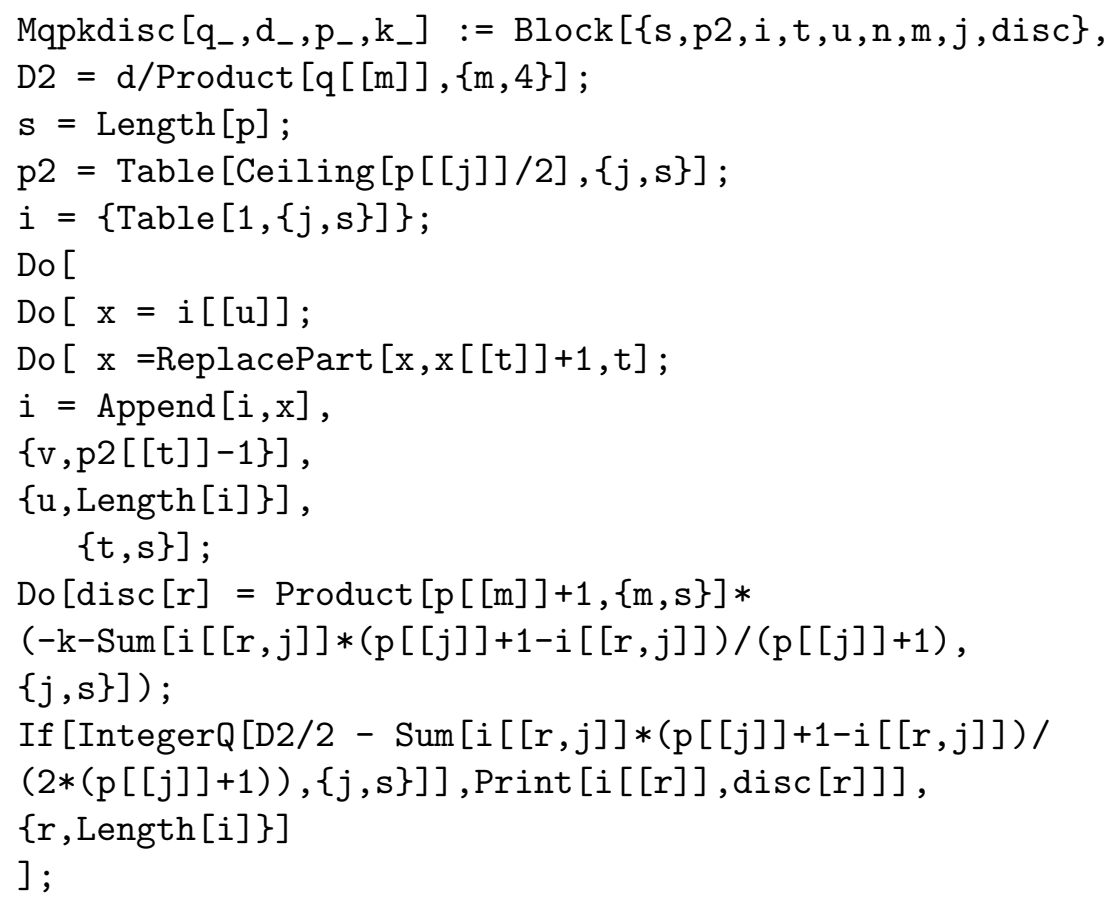

This procedure constructs the matrix associated to an $M_{\vec{p}, \vec{i}, k}$. To form such a matrix, we take the direct sum of the Cartan matrices associated to the $A_{p_{j}}$, and 
add a row/column which reflects which components intersect with the central vertex. We accomplish this by beginning with the identity matrix and then replacing portions of the rows/columns as appropriate. The variables cump and cumi are vectors for cumulative $\vec{p}$ and $\vec{i}$ so that the loops index correctly; the large Do-loop in the middle determines the correct Cartan matrix for the particular $A_{p_{j}}$.

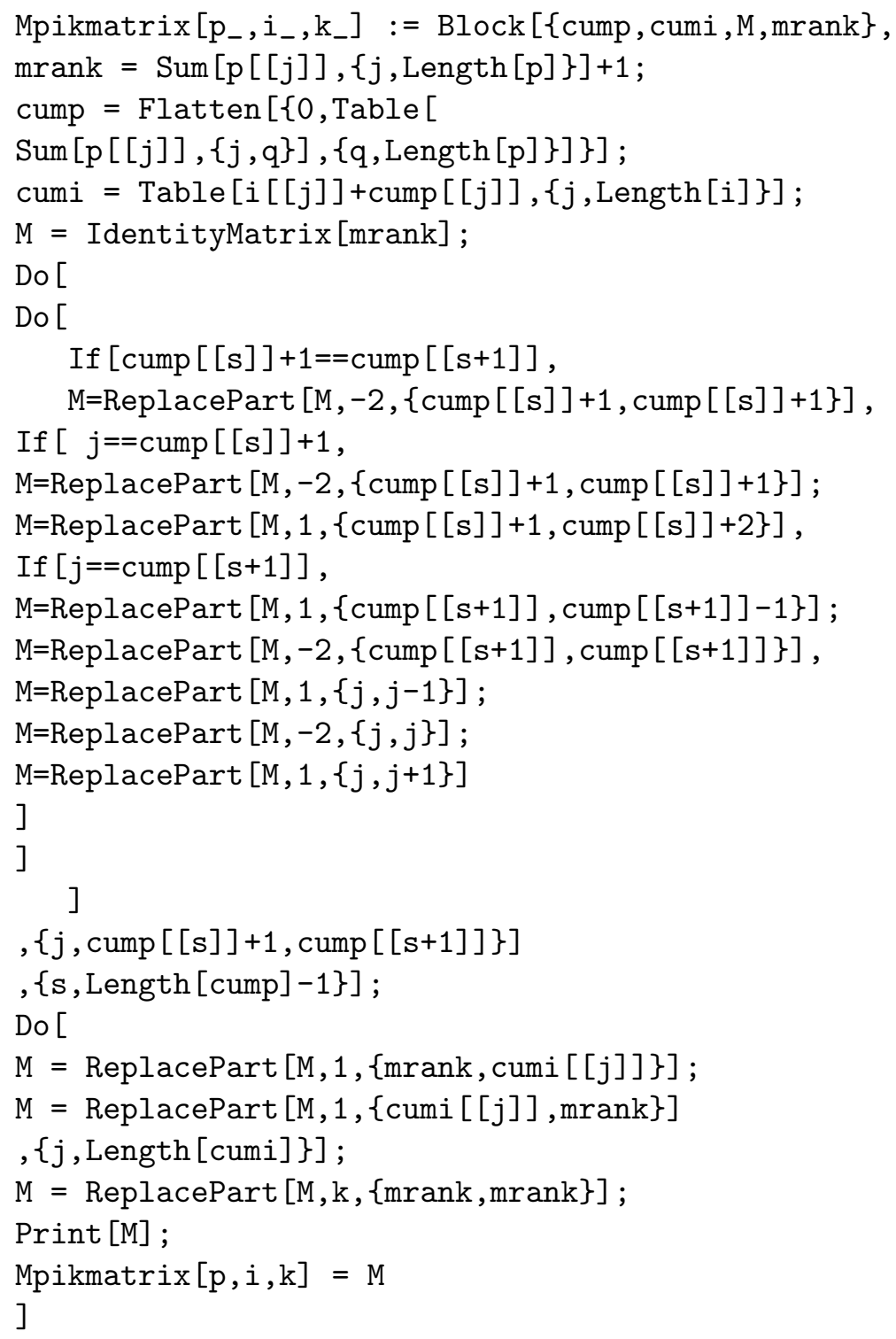




\section{BIBLIOGRAPHY}




\section{BIBLIOGRAPHY}

[Qhull] Barber, Brad, David Dobkin, and Hannu Huhdanpaa, Qhull, The Geometry Center, 1994.

[BPV] Barth, W., Peters, C. and A. Van de Ven, Compact Complex Surfaces, Ergebnisse der Mathematik, 3. Folge, Band 4, Springer-Verlag, Berlin, 1984.

[Brieskorn] Brieskorn, Egbert, The Milnor Lattices of the Exceptional Unimodular Singularities, Bonner Mathematische Schriften, No. 150, 1983.

[Brøndsted] Brøndsted, Arne, An Introduction to Convex Polytopes, GTM 90, Springer-Verlag, New York, 1983.

[Enriques I] Cossec, Francois R., and Igor V. Dolgachev, Enriques Surfaces I, Birkhäuser, Boston, 1989.

[Cox] Cox, David A., Picard Numbers of Surfaces in 3-Dimensional Weighted Projective Spaces, Mathematische Zeitschrift, 201 (1989), 183-189.

[Danilov] Danilov, V.I., The Geometry of Toric Varieties, Russian Math. Surveys, $33: 2$ (1978), 97-154.

[Dolg1] Dolgachev, Igor V., Mirror Symmetry for Lattice Polarized K3 Surfaces, Journal of Mathematical Sciences, vol. 81, No. 3, Sept. 10, 1996, Plenum Publishing Corporation.

[Dolg2] Dolgachev, Igor V., Polyhedral Hypersurfaces, handwritten notes, 1979.

[Dolg3] Dolgachev, Igor V., Weighted Projective Spaces, in "Group Actions and Vector Fields," Lect. Notes in Math. 956, 34-72, Springer-Verlag, New York, 1982.

[Dolg4] Dolgachev, Igor V., personal communication, 1997.

[VE] Fukuda, Komei and Ichiro Mizukoshi, Vertex Enumeration for Convex Polyhedra and Hyperplane Arrangements - Mathematica Package - Version 0.41 Beta, ftp.wri.com, 11.14.92.

[FL] Fukuda, Komei and Vera Rosta, Generating All Faces of Convex Polytopes:FaceLattice.m - Mathematica Package - Version 0.2 Beta, ftp.wri.com, 9.19.92. 
[Fulton] Fulton, William, Introduction to Toric Varieties, Annals of Mathematics Studies, Princeton University Press, Princeton, 1993.

[Humphreys] Humphreys, James E., Introduction to Lie Algebras and Representation Theory, Springer-Verlag, New York, 1972.

[Khovanskiı1] Khovanskiu, A.G., Hyperplane sections of polyhedra, toric varieties and discrete groups in Lobachevskii space, Func. Anal. App., 11 (1977), 289296.

[Miranda] Miranda, Rick, The Basic Theory of Elliptic Surfaces, ETS Editrice Pisa, ISBN 88-7741-462.

[Mir-Mor] Miranda, Rick, and David R. Morrison, The Number of Embeddings of Integral Quadratic Forms. II., Proc. Japan Acad., 62, Ser. A (1986), 0, 29-32.

[MirE] Miranda, Rick, personal communication.

[Mukai1] Mukai, Shigeru, Moduli of Vector Bundles on K3 Surfaces, and Symplectic Manifolds, Sugaku Expositions, 1:2 (1988), 139- 174.

[Mukai2] Mukai, Shigeru, On the Moduli Space of Bundles on K3 surfaces, I, in "Vector Bundles on Algebraic Varieties," Tata Inst. Fund. Res. Stud. Math., 11, 341-413, Tata Inst. Fund. Res., Bombay, 1987.

[Nikulin] Nikulin, V. V., Integral Symmetric Bilinear Forms and Some of Their Applications, Math. USSR Izvestiya, 14 (1980),1,103-167.

[Reid] Reid, Miles, Graded Rings over K3s, e-print, 7/96.

[YPG] Reid, Miles, Young Person's Guide to Canonical Singularities, in Algebraic Geometry (Bowdoin 1985), Proc. Symp. in Pure Math. 46, AMS 1987, 345414.

[Shioda] Shioda, T., On Elliptic Modular Surfaces, J. Math. Soc. Japan, 24 (1972), $20-59$.

[Tyurin] Tyurin, A. N., Cycles, Curves, and Vector Bundles on an Algebraic Surface, Duke Math. J., 54 (1987) No. 1, 1-26.

[Tyurin K3] Tyurin, A. N., Special 0-cycles on a Polarized K3 Surface, Izvestiya, 30 (1987) No.1, 129-138.

[Venkov] Venkov, B. A., Elementary Number Theory, p.115, Wolters-Noordhoff Publishing, Groningen (The Netherlands), 1970.

[Yonemura] Yonemura, Takashi,Hypersurface K3 Singularities, Tôhoku Math Journal, 42 (1990), 351-380. 


\section{ABSTRACT \\ Picard Lattices of Families of K3 Surfaces \\ by \\ sarah-marie belcastro}

Chair: Igor Dolgachev

It is a nontrivial problem to determine the Picard Lattice of a given surface; the object of this thesis is to compute the Picard Lattices of M. Reid's list of 95 families of Gorenstein K3 surfaces which occur as hypersurfaces in weighted projective space. Reid's list arises in many problems; here we look at an application to Mirror Symmetry.

One can define an analogue of Mirror Symmetry for Calabi-Yau threefolds for K3 surfaces. This analogue coincides with the "strange duality" for the 14 surface singularities of V. I. Arnold. Here we investigate the Mirror Symmetry of Reid's 95 families of surfaces; Arnold's singularities are on this list.

Denote a surface by $S$ and a mirror family by $\breve{S}$. Then we define a mirror family by

$$
\operatorname{Pic}(S)_{H^{2}(S, \mathbb{Z})}^{\perp}=\operatorname{Pic}(\check{S}) \perp U .
$$

By computing the Picard Lattice for each of these 95 surfaces, I am able to determine whether the mirror family for each one is also on Reid's list. 
I begin the thesis by reviewing the history of the problem, and then discuss the problems with computing the rank $\rho$ of the Picard Lattice. The bulk of Chapter 1 is devoted to exposition of lattices / quadratic forms and background on K3 elliptic surfaces. There is also an explanation of the computer programs I wrote to assist with the computations.

Chapter 2 concerns a conjecture by Reid on how to compute the Picard Lattice. I discuss this conjecture and investigate the new lattices $M_{\vec{p}, \vec{i}, k}$ which arise therein, and use the conjecture to re-compute the Picard Lattice for the 95 families.

Chapter 3 details the 95 calculations I made when computing the Picard lattices. In Chapter 4, I prove the existence of an index $d$ embedding of $\operatorname{Pic}(S)$ into $\operatorname{Pic} \mathbf{J}(S)$ for $S$ with multisection index $d$.

I include several appendices which contain the Mathematica code for my programs and various tables of quadratic forms and their values. 